\title{
The acquisition of cultural capital and habitus and their impact on the aca- demic achievement - a revised theory
}

\author{
Dissertation \\ Presented to the Faculty for Social Sciences, Economics, and \\ Business Administration at the University of Bamberg \\ in partial fulfillment of the requirements for the degree of \\ DOCTOR RERUM POLITICARUM \\ by \\ Frank Goßmann, M.A., \\ born November 29, 1979 in Würzburg, Germany
}




\begin{tabular}{|c|c|}
\hline Main supervisor: & $\begin{array}{l}\text { Prof. Dr. Steffen Schindler } \\
\text { University of Bamberg, Germany }\end{array}$ \\
\hline Co-supervisor: & $\begin{array}{l}\text { Prof. Dr. Corinna Kleinert } \\
\text { University of Bamberg, Germany }\end{array}$ \\
\hline Date of Submission: & June $13^{\text {th }}, 2020$ \\
\hline Date of Defense: & April 30 $0^{\text {th }}, 2021$ \\
\hline
\end{tabular}

Dieses Werk ist als freie Onlineversion über das Forschungsinformationssystem (FIS; https://fis.uni-bamberg.de) der Universität Bamberg erreichbar.

URN: urn:nbn:de:bvb:473-irb-503908

DOI: https://doi.org/10.20378/irb-50390 


\section{Abstract}

Even though it is well established that academic achievement is influenced by social origin, there are still open questions regarding the underlying mechanisms of this relation. In the quantitative sociology of education, cultural capital theory is often used to narrow this research gap. The cultural capital theory describes factors that mediate and thus explain the effect of social origin on academic achievement. However, there are different interpretations of the theory that are subject to some criticism. Bourdieu's works, although they serve as the origin of many approaches to solutions, are criticized for unclear definitions and hypotheses as well as insufficient empirical evidence. The further developments and interpretations of the theory that are meanwhile in common use are also subject to considerable criticism as empirical applications show limited explanatory power, the theoretical focus on high culture is too narrow, and the interrelated concept of habitus is ignored. Although the different readings of the theory give rise to different criticisms, they offer, together and in complement with related disciplines, the elements of a more comprehensive theory. This thesis specifies such an integrating theory that is testable with quantitative empirical methods. This is not achieved by developing a precise interpretation of Bourdieu's work or other existing theories. Rather, this revised theory consists of elements of different interpretations of the cultural capital theory, but also from psychology and educational research. The aim of the thesis is to derive a theory with a higher explanatory power than the common theory and to demonstrate this in direct empirical comparison. The theory referred to as common theory describes the elaboration based on DiMaggio (1982) and the extension by Crook (1997) and de Graaf et al. (2000).

First, a theory is specified based on the answers to three basic questions. (1) How can the central terms of a useful theory of cultural capital and habitus be defined? (2) How are cultural capital and habitus acquired? (3) How does cultural capital and habitus affect academic achievement? The answers to these questions result in the revised theory. On this basis, hypotheses are formulated on the acquisition of cultural capital and habitus and their effect on academic achievement. The theory explains the effect of social 
origin on academic achievement via social differences in skills, knowledge, and motivational factors. They have distinctive effects or affect the ability to learn and follow school lessons or serve as explicit assessment criteria. In the common theory, social inequalities in academic achievement are accounted for by differences in high cultural characteristics and reading activities. High cultural characteristics function as means of social distinction of the upper classes, whereas reading furthers learning.

Second, the hypotheses derived from the revised theory of cultural capital and habitus are tested empirically. The re-specification of the concepts is complemented by adapted operationalizations. Multiple-group path analyses using NEPS Starting Cohort 3 data are applied to test the hypotheses. Maximum likelihood estimators with robust standard errors are used and sampling strata and clustering in schools are accounted for. To avoid biased results due to item-nonresponse, multiple imputation by chained equations is utilized, creating 100 imputed data sets. Cultural capital and habitus are conceptualized as mediators of the social origin effect on academic achievement. Hence, the following question arises: (4) Do the postulated causal pathways make a significant contribution to the explanation of social inequalities in academic achievement? Based on the multiple-group path analyses, these indirect effects are tested.

Third, the explanatory power of the revised and the common theory are compared regarding social inequalities in academic achievement. The underlying claim is that only if the revised theory performs better than the common theory, it is legitimate. Thus, the following question has to be answered: (5) Does the revised theory show a higher explanatory power with regard to social inequalities in academic achievement than the common theory? Therefore, the standardized indirect effects of social origin on academic achievement are compared. Moreover, the squared semi-partial correlations of social origin and academic achievement are contrasted when the mediating variables of the respective models are controlled.

Summarized, the revised theory explains the effect of social origin on academic achievement considerably better than the common theory. The higher explanatory power of 
the revised model is based on the consideration of passive and active linguistic skills. Moreover, social distinction measured by high cultural activities does not explain the effect of social origin on academic achievement when linguistic skills are considered. Future research could address alternative characteristics that might serve as means of social distinction, for example high cultural knowledge or language use. Another field of future research could be the social differences in parenting practices that account for social differences in skills, knowledge, and motivation that affect academic achievement.

Keywords: Cultural Capital, Habitus, Academic Achievement, Social Inequality 


\section{Acknowledgments / Danksagung}

Diese Dissertation entstand größtenteils während meiner Zeit am NEPS bzw. LIfBi, in der ich viele wertvolle Erfahrungen sammeln konnte.

Ich danke insbesondere meinem Doktorvater, Prof. Dr. Steffen Schindler, für seine Betreuung, seine offene, unterstützende Art und die inhaltlichen Anregungen. Mein Dank gilt auch meinen beiden weiteren Gutachterinnen Prof. Dr. Corinna Kleinert und Prof. Dr. Sandra Buchholz für ihre Unterstützung.

Dem leider bereits verstorbenen Prof. Dr. Volker Stocké möchte ich hier gerne gedenken, der mich nach meinem Studium als wissenschaftlicher Mitarbeiter eingestellt hat.

Die Zusammenarbeit und die vielen hilfreichen und anregenden Diskussionen mit meinen Kolleginnen und Kollegen haben meine Dissertation sehr bereichert. Mein besonderer Dank gilt daher Kerstin Hoenig, Vanessa Obermeier, Sebastian Wenz, Hannah Steinberg, Michael Bayer, Markus Zielonka, Tobias Linberg und Jacqueline Lettau. Gleiches gilt für die Teilnehmerinnen und Teilnehmer des BAGSS Kolloquiums, insbesondere Friederike Schlücker.

Ein ganz besonderer Dank gilt meiner Familie, zuvorderst meiner Frau Solange, die mich nicht nur jederzeit unterstützt und motiviert hat, sondern auch inhaltlich mit mir diskutiert und mir familiär den Rücken freigehalten hat. Auch meine Eltern und Schwiegereltern haben mir hier sehr geholfen. Meine Kinder Fabian und Dana haben auf viel Zeit mit mir als Vater verzichten müssen, die gemeinsame Zeit war mir aber immer ein wertvoller und geschätzter Ausgleich. Ich bin sehr glücklich darüber, dass ich jetzt, da unser Sohn Felix unsere Familie weiter bereichert, diese Arbeit erfolgreich abschließen konnte. 


\section{Contents}

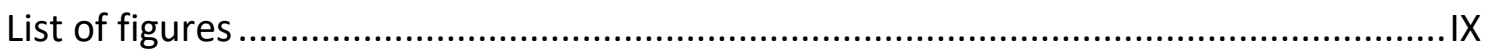

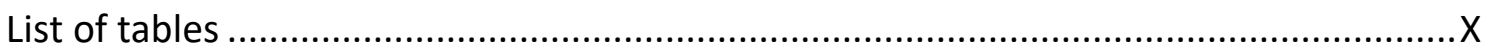

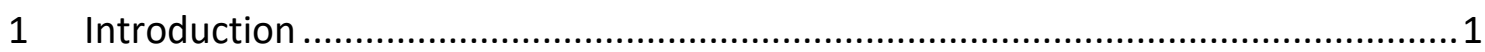

2 Theoretical framework: Cultural capital and habitus ...........................................

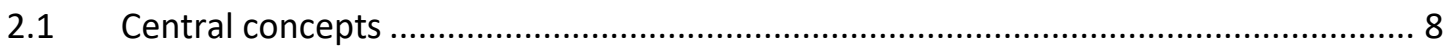

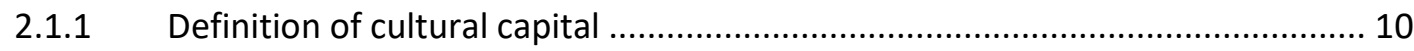

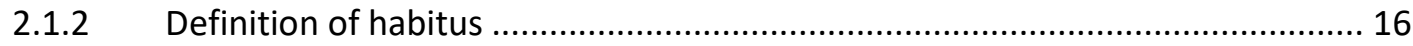

2.1.3 Disentangling cultural capital, habitus, and practice ........................................ 20

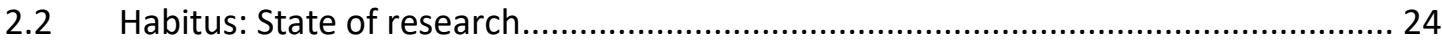

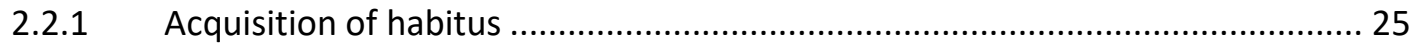

2.2.2 The impact of habitus on academic achievement ............................................. 35

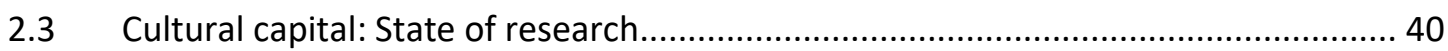

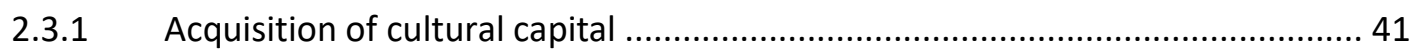

2.3.2 The impact of cultural capital on academic achievement ................................ 47

2.4 Contrasting the common and the revised theory................................................... 51

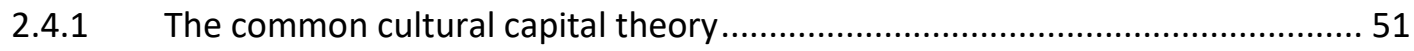

2.4.2 A revised theory of cultural capital and habitus: hypotheses ............................ 55

2.4.3 Differences between the common and the revised theory............................... 64

3 Explaining social inequalities in academic achievement: An empirical comparison...

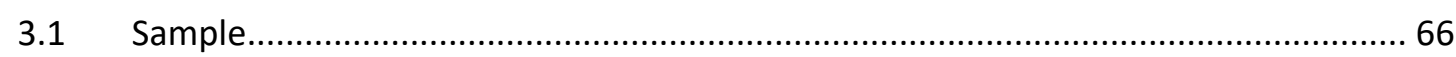

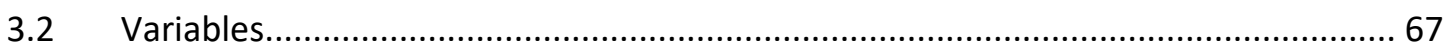

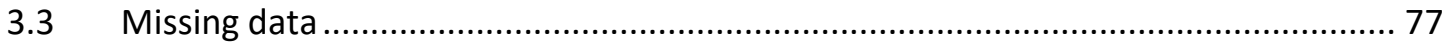

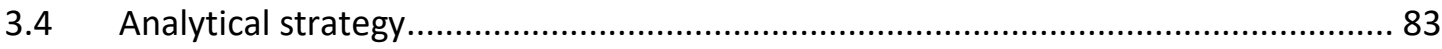

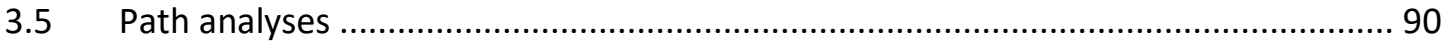

3.5.1 Model specification - single-group path analyses ............................................ 91

3.5.2 Sample size and statistical power - single-group path analyses ........................ 94

3.5.3 Model fit evaluation - single-group path analyses........................................... 94 
3.5.4 Model re-specification - multiple-group path analyses .................................. 98

3.5.5 Sample size and statistical power - multiple-group path analyses .................. 100

3.5.6 Model fit evaluation - multiple-group path analyses..................................... 103

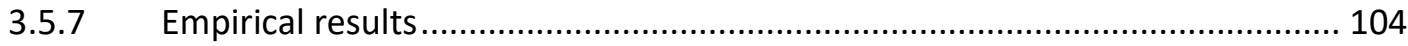

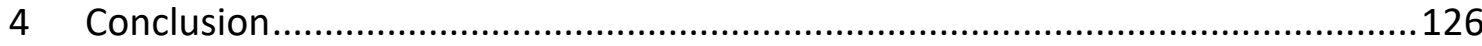

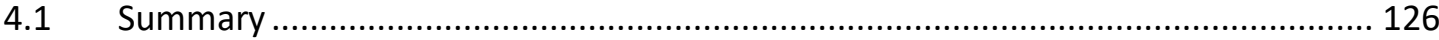

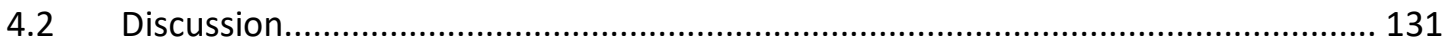

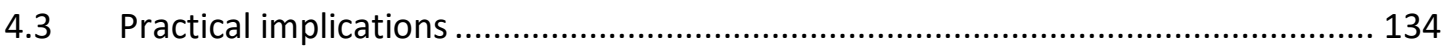

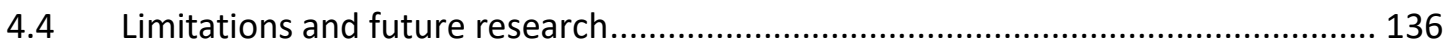

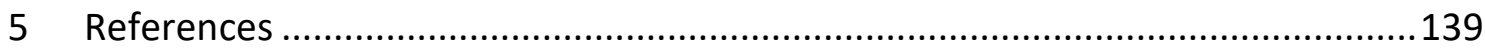

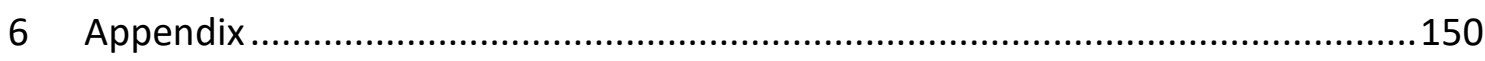

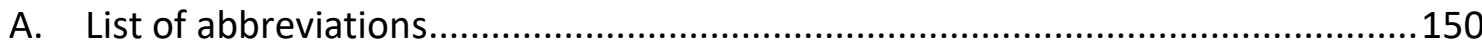

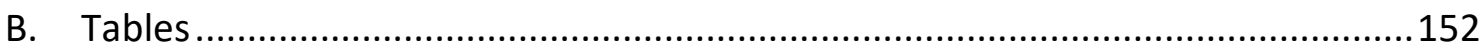




\section{List of figures}

Figure 1: Path model of the common cultural capital theory.....................................53

Figure 2: Path diagram of a revised theory of cultural capital and habitus ...................57

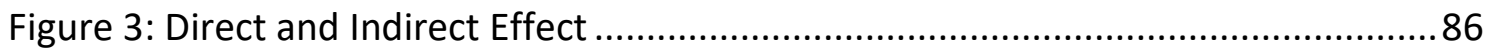

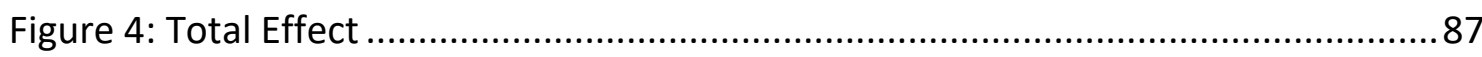

Figure 5: Venn diagram to illustrate the squared semi-partial correlation, based on

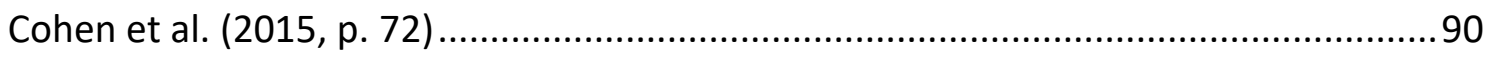

Figure 6: Specification of the common cultural capital model - single-group path analysis

Figure 7: Specification of the revised theoretical model - single-group path analysis.. 93 Figure 8: Specification of the common cultural capital model - multiple-group path

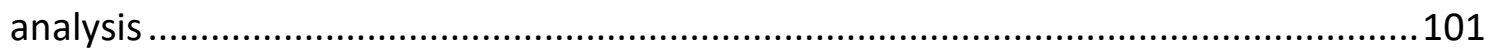

Figure 9: Specification of the revised theoretical model - multiple-group analysis ....102

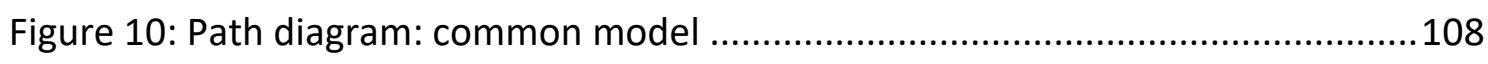

Figure 11: Direct, indirect, and total effects of parental education on mediator variables

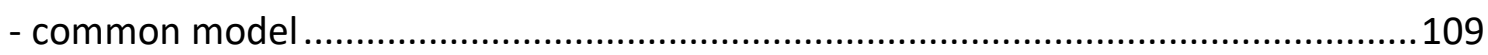

Figure 12: Indirect effects of parental education on students' grades - common model 111

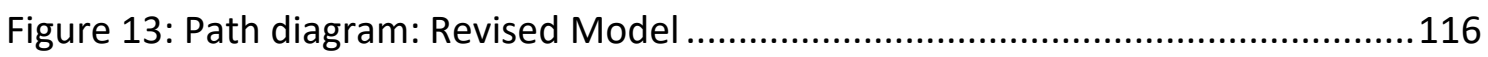

Figure 14: Direct, indirect, and total effects of parental education on mediator variables - revised model. 117

Figure 15: Indirect effects of parental education on students' grades - revised model 120

Figure 16: Comparison of the total, direct, and total indirect effects of parental education on students' grades

Figure 17: Comparison of the semi-partial correlations between students' average grades and parental education 125 


\section{List of tables}

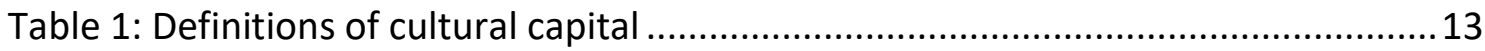

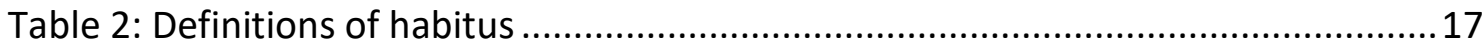

Table 3: Systematic overview of definientia of cultural capital and habitus..................21

Table 4: Descriptive values of dependent and independent variables ........................76

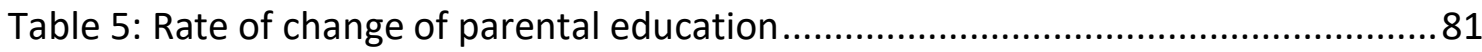

Table 6: Comparison of descriptive statistics of observed and imputed data ...............82

Table 7: Model fit indices of the single-group path analyses ......................................95

Table 8: Means and standard deviations of the mediating variables by type of school 98

Table 9: Model fit indices of the multiple-group path analyses 103

Table 10: Overview of the results on the acquisition of cultural capital - common theory

Table 11: Overview of the results on the impact of cultural capital on academic achievement - common theory

Table 12: Overview of the results on the acquisition of habitus and cultural capital revised theory

Table 13: Overview of the results on the impact of habitus and cultural capital on academic achievement - revised theory.

Table 14: Cross table of the highest social class position (EGP) and the highest formal education of the parents. 152

Table 15: Standardized residual covariances - common model - single-group analysis

Table 16: Standardized residuals covariances - revised model - single-group analysis154 Table 17: Standardized residual covariances - revised model - multiple-group path analysis (non-final) - no tracking

Table 18: Standardized residual covariances - revised model - multiple-group path analysis (non-final) - lower and intermediate track 156 
Table 19: Standardized residual covariances - revised model - multiple-group path analysis (non-final) - higher track.

Table 20: Standardized residual covariances - revised model - multiple-group path analysis (final) - no tracking

Table 21: Standardized residual covariances - revised model - multiple-group path analysis (final) - lower and intermediate track 159

Table 22: Standardized residual covariances - revised model - multiple-group path analysis (final) - higher track 160

Table 23: Standardized residual covariances - common model - multiple-group path analysis (final) - no tracking

Table 24: Standardized residual covariances - common model - multiple-group path analysis (final) - lower and intermediate track

Table 25: Standardized residual covariances - common model - multiple-group path analysis (final) - higher track

Table 26: Results of the path analyses: Common model and revised model

Table 27: Direct, indirect, and total effects of parental education on mediating variables - common model

Table 28: Direct, indirect, and total effects of parental education on students' grades common model

Table 29: Differential effects on students' grades across types of school - common model

Table 30: Direct, indirect, and total effects of parental education on mediating variables - revised model 180

Table 31: Direct, indirect, and total effects of parental education on students' grades revised model 185

Table 32: Differential effects on students' grades across types of school - revised model

Table 33: Cross table: Parental education and type of school 193

Table 34: Pairwise correlations of the observed data 193 


\section{Introduction}

Findings in the sociology of education consistently show that educational success is to a large extent determined by social origin (see for example Alba, Handl, \& Müller, 1994; D. Baker, Esmer, Lenhardt, \& Meyer, 1985; Blossfeld \& Shavit, 1993; Buchholz \& Pratter, 2017; Diefenbach, 2002; W. Müller, 1998; Schindler \& Lörz, 2011). A very influential theory dealing with the mechanisms behind those educational inequalities is Bourdieu's cultural capital theory (1977a). An important focus of the theory is the explanation of the assessment of students by teachers and, hence, academic achievement. Although Bourdieu's concept is often referred to, it has also been criticized:

(1) The central term cultural capital is not clearly defined (A. Sullivan, 2001, p. 896). Hence, there are substantially diverging meanings of cultural capital. This complicates a fruitful discussion about the role of cultural capital in educational inequality. As the term thus defined is elusive, it is almost impossible to test and criticize the theory empirically. Criticism and empirical falsification can be simply rejected arguing that the concept of cultural capital applied is not appropriate.

(2) Bourdieu's writings lack clear hypotheses (Jæger \& Breen, 2016; A. Sullivan, 2001). He refrained to give a specific description of how cultural capital affects educational success. Therefore, the relations of the explained facts and the explaining factors remain cloudy, which hampers further scientific discourse and complicates testing using empirical methods.

(3) Bourdieu's empirical analyses are insufficient to underpin his theory (A. Sullivan, 2001). More specifically, he does not present quantitative analyses that consistently show that cultural capital is transmitted from parents to their children, that children's cultural capital affects their educational success, and that educational attainment impacts occupational status. 
However, Bourdieu's work influenced a large body of research (Kingston, 2001; Lareau \& Weininger, 2003). Due to the unclear definition of cultural capital and the vague hypotheses, very different theories named cultural capital are developed in educational research. The most common line of research in quantitative sociology of education goes back to DiMaggio (1982) and conceptualizes cultural capital as affinity towards, participation in, or knowledge about high culture (Goldthorpe, 2007, p. 91; Kingston, 2001, pp. 91-92; Lareau \& Weininger, 2003, 568, 574). DiMaggio (1982) introduces cultural capital as an explanatory factor of academic achievement in addition to skills. Oftentimes, studies following this view extend cultural capital by an additional dimension introduced by Crook (1997): activities that help to develop skills, typically reading activities (Goldthorpe, 2007, pp. 90-91). In the following, this common line of research is referred to as common theory. Although this widespread reading of the cultural capital theory is very popular, it is also subject to criticism:

(1) The common theory shows only little explanatory power (Kingston, 2001, p. 94). The connection between social origin and educational success is explained only to a small extent.

(2) The common interpretation of cultural capital as affinity towards, participation in, or knowledge about high culture is too narrow (Lareau \& Weininger, 2003, pp. 568-569). Lareau and Weininger (2003, pp. 568-569) argue that Bourdieu does not exclusively focus on high culture. Moreover, they claim that the common differentiation of cultural capital from any kind of ability or skills is not supported by Bourdieu's work. Indeed, Bourdieu explicitly defines "linguistic and cultural competence" (Bourdieu, 1977a, p. 494) as constitutive for cultural capital.

(3) The common cultural capital theory ignores the concept of habitus (Dumais, 2002; Gaddis, 2013). At least conceptionally, cultural capital and habitus bear a high explanatory potential in terms of educational inequalities. However, due to 
vague definitions the differentiation of cultural capital and habitus and their interrelation remain unclear. Moreover, investigating the impact of cultural capital on educational success and ignoring the role of habitus can lead to invalid conclusions regarding the causal mechanisms as, from a theoretical perspective, cultural capital correlates with habitus.

Nonetheless, cultural capital theory contains valuable elements that can be used as a basis. The literature of recent years has been going in different directions in this respect. Some studies deviate from the common approach of cultural capital to clarify mechanisms that underlie the influence of cultural capital on academic achievement. Building on the common theory, they include skills respectively knowledge as explanatory variables how social origin affects academic achievement (Mikus, Tieben, \& Schober, 2020; A. Sullivan, 2001). Results show that linguistic skills and knowledge are related to cultural capital measures and that they play a crucial role for the explanation of academic achievement. Regarding the causal mechanisms through which cultural capital affects academic achievement, these findings are of central importance. However, the acquisition of skills and knowledge is explained only by reading ${ }^{1}$ and rather rare high cultural activities like going to the museum or theater. Even though it is plausible that such activities stimulate the development of skills and knowledge, the restriction to exclusively these cultural explanatory factors is not confirmed by other research results. Findings from the qualitative sociological work of Lareau (2002), for example, as well as psychological studies (for example Hoff, 2006) and studies from educational research (for example Hart \& Risley, 1995) offer very good starting points for a more comprehensive cultural explanation by adding familial socialization and parenting practices as explanatory factors.

The present doctoral thesis builds on the assumption that the theories of cultural capital and habitus bear a high explanatory potential regarding social inequalities in educational success. Therefore, the different concepts of cultural capital are critically assessed and

\footnotetext{
${ }^{1}$ Additionally, Sullivan (2001) explains the acquisition of skills and knowledge by watching sophisticated television program.
} 
restructured under consideration of a wider, interdisciplinary perspective to obtain a useful theory as defined by Opp (compare Opp, 2005, pp. 216-221): (1) The terms must be clearly and unambiguously defined. (2) The hypotheses have to be clearly formulated. The explaining factors and the explained facts must be clear. Moreover, the theory must describe the interrelation between explaining factors and explained facts. (3) The theory must prove itself empirically. (4) The theory should inform about appropriate interventions.

First, a useful theory is put forward that can be tested by quantitative empirical methods. This is illustrated by the critical answers to the following three questions.

(1) How can the central terms of a useful theory of cultural capital and habitus be defined?

With the goal of deriving disjoint and complementary concepts, the definitions of cultural capital and habitus of Bourdieu and other important researchers are not only interpreted, but also explicated.

(2) How are cultural capital and habitus acquired?

(3) How does cultural capital and habitus affect academic achievement?

Based on the revised definitions, hypotheses are formulated that are informed by interdisciplinary findings. To acquire a comprehensive theoretical basis, studies primarily from sociology, psychology, and educational research are considered. Apart from clear definitions of terms, the description of causal mechanisms allows meaningful hypotheses to be formulated and appropriate operationalizations to be chosen or developed. The resulting theory is called revised theory in the following.

Second, the hypotheses of the revised theory of cultural capital and habitus are tested empirically. In the context of cultural capital, Bourdieu explains educational inequalities essentially through socially driven differences in the assessment of students by teachers. Thus, many researchers investigate the effect of cultural capital on school grades (for 
example Bourdieu, 1984; DiMaggio, 1982; Rössel \& Beckert-Zieglschmid, 2002). Hence, the central dependent variable is students' average grade in German and mathematics. Since the definitions of the explanatory factors are being revised, the operationalizations are also being adapted accordingly. Path analyses are used, as the hypotheses form complex causal chains through which academic achievement is affected. In this manner, the system of hypotheses can be tested using one model instead of a series of other multivariate methods like regression analysis.

Even more important than the confirmation of the single hypotheses concerning the acquisition of cultural capital and habitus and their effect on academic achievement, however, is the answer to the superordinate question addressed by the theory:

(4) Do the postulated causal pathways make a significant contribution to the explanation of social inequalities in academic achievement?

As cultural capital theory is assumed to explain the effect of social origin on academic achievement, it must be shown that the effect of social origin on academic achievement is mediated by explanatory factors outlined in the theory. Path analysis allows testing those indirect effects.

Third, the explanatory power of the revised and the common theory are compared regarding social inequalities in academic achievement. Even though it is criticized in the abovementioned way, a mainstream or common theory of cultural capital exists (compare Goldthorpe, 2007; Lareau \& Weininger, 2003). To justify a revised theory, it must perform better than the common cultural capital theory. The central criterion of performance is defined by the aim of the theories: the explanatory power with regard to social inequalities in academic achievement. The theory with the higher explanatory power should be preferred. Therefore, a further question arises:

(5) Does the revised theory show a higher explanatory power with regard to social inequalities in academic achievement than the common theory? 
Hence, the standardized indirect effects of social origin on academic achievement are compared. Additionally, the squared semi-partial correlations between the indicator of social origin and academic achievement are contrasted when the mediating cultural capital and habitus variables are controlled.

In the end, the empirical part of the thesis is a comparison of the revised and the common theory. In order to enable a comprehensive comparison, both theories are empirically tested and then compared in the crucial points described above.

For the empirical analyses, the NEPS (National Educational Panel Study) Starting Cohort 3 data $^{2}$ (Blossfeld, Roßbach, \& Maurice, 2011) is used as it contains a great variety of relevant measures. To avoid biased results by item-nonresponse, missing data is multiply imputed by chained equations. Besides the variables of the analytical models, a variety of auxiliary variables is used and 100 completed data sets are generated.

In short, the aim of the thesis is to derive a cultural capital theory with a higher explanatory power regarding social inequalities in academic achievement than the common theory and to demonstrate this in direct empirical comparison. Cultural capital theory describes factors that mediate and thus explain the effect of social origin on academic achievement. However, the commonly used interpretation of the theory in the sociology of education has a special theoretical focus, empirical applications show limited explanatory power, and the related concept of habitus is ignored. Even though the different readings of the theory give rise to different criticisms, they offer, together and in complement with related disciplines, the elements of a more comprehensive theory. This thesis specifies such an integrating theory.

The thesis is structured as follows. Chapter 2 provides the theoretical basis of the thesis. A variety of definitions of cultural capital and habitus are collected, summarized and

\footnotetext{
2 This paper uses data from the National Educational Panel Study (NEPS): Starting Cohort Grade 5, doi: 10.5157/NEPS:SC3:7.0.0. From 2008 to 2013, NEPS data was collected as part of the Framework Program for the Promotion of Empirical Educational Research funded by the German Federal Ministry of Education and Research (BMBF). As of 2014, NEPS is carried out by the Leibniz Institute for Educational Trajectories (LIfBi) at the University of Bamberg in cooperation with a nationwide network.
} 
systemized. From this synopsis, the revised definitions are derived. Based on these definitions, findings with regard to the acquisition of habitus and cultural capital and the impact of habitus and cultural capital on academic achievement are presented. Additionally, a revised theory is derived drawing on the reviewed research, clarifying the corresponding hypotheses. In order to compare the revised theory with the common theory, the common theory is also presented.

In chapter 3 , the common and the revised theory are tested and compared. The chosen data are described and the choice of the model variables is justified. Missing data are analyzed and the multiple imputation method chosen to handle the effect assumed of data missing at random is presented. The analytical strategy and model specifications are described. Multiple-group path analyses are conducted to handle the problem of different school types that affect the strength of the explaining factors in an ordinary single-group path model. This consideration of school type effects is implemented on both, common and revised model. Sample size and statistical power are considered and empirical results are reported.

Chapter 4 concludes with a summary of the theories, the corresponding empirical results, a critical discussion, and practical implications. 


\section{Theoretical framework: Cultural capital and habitus}

In the chapters 2.1.1 and 2.1.2, definitions of cultural capital and habitus are gathered, interpreted, and explicated. Based on this clarification of terms, the different definitions are systemized and definitions are derived as a conceptual basis for the revised theory in chapter 2.1.3. Next, the state of research on the concepts underlying the revised definitions is presented (chapters 2.2 and 2.3). The structure of this description of the state of research is guided by the revised definitions of habitus and cultural capital. In chapter 2.4 , the theories that are tested and compared empirically in chapter 3 are described. First, the common theory of cultural capital is outlined as it serves as a frame of reference for the revised theory. Then, the revised theory is specified based on the state of research depicted before. Finally, both theories are briefly contrasted.

\subsection{Central concepts}

The concept of cultural capital introduced by Bourdieu has clearly influenced research in the quantitative sociology of education. However, he is criticized for giving vague definitions (for example Jæger \& Breen, 2016, p. 1080; A. Sullivan, 2001, p. 894). This ambiguity is carried forward in the subsequent literature inspired by the writings of Bourdieu and Passeron (for example 1977a; 1977) and leads to a variety of theories that are labeled as cultural capital, but use different concepts of cultural capital (Goldthorpe, 2007; Lareau \& Weininger, 2003). However, a fundamental prerequisite for a useful theory is a clear terminology (Opp, 2005, pp. 134-135). Vague and ambiguous concepts hamper the scientific communication and the possibility to criticize a theory as the meaning of theoretical statements becomes unclear. Therefore, it is getting harder to test a theory based on vague and ambiguous concepts empirically. If the aim is to formulate meaningful and testable theories that ideally inform us about potential interventions to reduce educational inequality, terms have to be clarified first.

In his textbook on the methodology in the social sciences Opp (2005, pp. 138-140) described two methods to achieve that goal: interpretation and explication. Interpretation means that terms are specified in the sense of the originator's intention. In contrast, the 
method of explication aims at specifying unclear terms so that they are suitable to formulate a theory that is informative and true (Opp, 2005, p. 139). Terms specified by explications are not necessarely consistent with the originator's intention. Both methods, interpretation and explication, are applied in the following. Of course the following interpretations and explications are not the only possible ones. As one of the goals of this thesis is to formulate a useful theory, it is not necessary to capture all potential meanings. Quite the opposite could be the case as including all potential meanings might lead to unclear or inconsistent terms. Ultimately, reducing the uncertainty by interpretation and explication facilitates building a useful theory that is accessible to criticism and further development.

However, the thesis pursues the potentially competing goals to give clear definitions of cultural capital and habitus and to build a theory with a high explanatory power regarding educational inequality. Thus, some problems occur: The first problem refers to the consequences of the clarification of concepts. Overcoming the ambiguouity of the concepts of cultural capital and habitus inevitably results in the exclusion of potentially important explanatory factors from the theoretical framework. However, cultural capital and habitus are complementary concepts (Dumais, 2002, p. 45) but according to some definitions of these terms, the concepts overlap (see chapter 2.1.3). This is the second problem. Hence, to obtain clear, consistent, disjoint, and complementary concepts that form a proper theoretical basis, the procedure is as follows: First, a variety of substantially different definientia ${ }^{3}$ of cultural capital and habitus are gathered from literature, clarified by interpretation and explication, and summarized (chapters 2.1.1 and 2.1.2). Secondly, the definientia of cultural capital and habitus are compared in a structured form (chapter 2.1.3). Considering this structured comparison, revised definitions of cultural capital and habitus are derived that form the conceptual basis of this thesis.

\footnotetext{
${ }^{3}$ Definitions consist of two parts (compare Opp, 2005, p. 108): the definiens (plural: definientia) and the definiendum (plural: definienda). The definiens is the expression that specifies respectively defines the definiendum.
} 


\subsubsection{Definition of cultural capital}

In the following, a variety of definitions of cultural capital is cited, interpreted, and explicated. The focus here is exclusively on the concept of cultural capital, which Bourdieu (1986, p. 243) defines as embodied cultural capital. Objectified and institutionalized cultural capital are not the subject of the study. This is in line with the definitions of cultural capital typically found in quantitative empirical sociology of education (compare Goldthorpe, 2007; Lareau \& Weininger, 2003). As a starting point, definitions by Bourdieu are considered first. Then the overview is supplemented by other important definitions.

\section{Bourdieu's notion of cultural capital}

Bourdieu gives different definitions of cultural capital: "long-lasting dispositions of the mind and body" (1986, p. 243), "culture, cultivation, Bildung" (1986, p. 244), a code, that is needed in order to decipher cultural goods and to internalize cultural capital (1977a, p. 493), "instruments for the appropriation of symbolic wealth socially designated as worthy of being sought and possessed" (Bourdieu, 1977a, p. 488), "linguistic and cultural competence" (Bourdieu, 1977a, p. 494), as well as familiarity with the dominant culture (1977a, p. 494).

Dispositions of the mind (1986, p. 243) could be interpreted according to Bourdieu's earlier work as "predisposition, tendency, propensity, or inclination" (Bourdieu, 1977b, p. 214). This explanation given by Bourdieu suggests that disposition might refer to a tendency to a certain behavior or action. To put it in more comprehensible words, tendency can be explained in terms of Ajzen's definition of an attitude toward a behavior as "positive or negative evaluation of performing [...] [a certain] behavior" (Ajzen, 1988, p. 117). The term dispositions of the body $(1986$, p. 243) could refer to posture, gestures, and facial expressions that are typical for the social class position of a person (compare Bourdieu \& Passeron, 1971, p. 114, 1977, p. 162). 
Furthermore, Bourdieu gives some synonyms for cultural capital: "culture, cultivation, Bildung" (1986, p. 244). Hence, Bourdieu paraphrases cultural capital with terms implying general knowledge, knowledge in general, and skills. The former of which can be termed as educational canon or knowledge that everybody should have.

The notion of the code is equivalent to "the instruments for the appropriation of symbolic wealth" (see 1977a, p. 488):

\begin{abstract}
"In view of the fact that the apprehension and possession of cultural goods [...] are possible only for those who hold the code making it possible to decipher them or, in other words, that the appropriation of symbolic goods presupposes the possession of the instruments of appropriation [...]"
\end{abstract}

Symbolic goods are cultural products that are defined as valuable by the cultural elite of a society. Essentially, a cultural product is anything that is created by humans. To appropriate these cultural products means to comprehend and acquire their content. However, the messages of cultural products are encoded by a system of words, signs, or symbols representing the messages. Hence, possessing the code respectively the instruments for their appropriation means to have the cognitive skills and the knowledge that are necessary to decode and comprehend the cultural products defined as valuable. Moreover, the code refers to skills and knowledge that are needed to understand school lessons (1977a, p. 493). Bourdieu argues that school lessons presuppose specialized prior knowledge, that is, students fully understand school lessons only if they possess this prior knowledge. Furthermore, teaching is primarily language-based. Therefore, Bourdieu regards linguistic skills as a central component of the code (1977a, p. 494).

Bourdieu does not define the notion cultural competence. It can be interpreted that cultural competence is a synonym for the code or at least part of the code in terms of the cognitive skills and the knowledge that are necessary to comprehend cultural products as he, for example, states that cultural competence is the condition for "the reception of a pictorial message" (1977a, p. 493). 
Moreover, in Bourdieu's writings, the term familiarity with the dominant culture remains unclear. It is plausible to explicate familiarity as good knowledge (compare also A. Sullivan, 2001, p. 896). In addition, Bourdieu argues that the acquisition of a substantial amount of cultural knowledge in the family is commonly associated with some kind of ease regarding the dominant culture (Bourdieu \& Passeron, 1977, p. 124) that allows for a natural and composed dealing with culture. This could be termed as confidence in one's own knowledge.

Summarized, Bourdieu's notion of cultural capital refers to very different concepts: positive or negative evaluation of performing a certain behavior (Bourdieu, 1986), body language, that is posture, gestures, and facial expressions (Bourdieu, 1986), cognitive and linguistic skills (Bourdieu, 1977a, 1986), knowledge (Bourdieu, 1977a, 1986), and confidence in one's own knowledge (Bourdieu \& Passeron, 1977).

\section{Further development of the term cultural capital}

Table 1 gives an overview regarding the definitions of cultural capital from influential studies as well as from some more recent works. The aim of this table is to show the conceptual variety of definitions of cultural capital. Therefore, it is not exhaustive.

Overall, the definitions of cultural capital are diverse, oftentimes abstract and unclear. In the following, the presented definitions are interpreted when necessary. Finally, the elements of the definitions of cultural capital are summarized.

DiMaggio defines cultural capital according to Bourdieu as "[...] instruments for the appropriation of symbolic wealth socially designated as worthy of being sought and possessed" (original Bourdieu, 1977a, p. 488; 1982, p. 190). In contrast to Bourdieu, who basically conceived those "instruments" as cognitive skills and knowledge (see above), DiMaggio refers to Weber's elite status culture (Weber, 1978, p. 932), that is the "distinctive cultural traits, tastes, and styles" (1982, pp. 189-190; Lareau \& Weininger, 2003, p. 574) of groups with a high social status. Distinctive cultural traits could be conceived of as any characteristic that is typical only for a certain group of people, tastes as positive 
evaluations of certain objects, and styles as the way of behaving and the physical appearance. However, according to Weber status culture is "a [group] specific style of life" (Weber, 1978, p. 932). Hence, elite status culture is the style of life of groups with a high social status.

Table 1: Definitions of cultural capital

"[...] instruments for the appropriation of symbolic wealth socially designated as worthy of being sought and possessed" (original Bourdieu, 1977a, p. 488; DiMaggio, 1982, p. 190) in terms of "distinctive cultural traits, tastes, and styles" (DiMaggio, 1982, pp. 189-190) of elite status groups

"institutionalized, i.e., widely shared, high status cultural signals (attitudes, preferences, formal knowledge, behaviors, goods and credentials) used for social and cultural exclusion"(Lamont \& Lareau, 1988, p. 156)

The capability to comply with "the informal academic standards by which teachers reward more general skills, habits, and styles"(Farkas, Grobe, Sheehan, \& Shuan, 1990, p. 127)

"proficiency in and familiarity with dominant cultural codes and practices - for example, linguistic styles, aesthetic preferences, styles of interaction."(Aschaffenburg \& Maas, 1997, p. 573)

"parents' cultural [...] resources become forms of capital when they facilitate parents' compliance with dominant standards in school interactions. In particular, cultural capital includes parents' large vocabularies, sense of entitlement to interact with teachers as equals, time, transportation, and child care arrangements to attend school events during the school day." (Lareau \& Horvat, 1999, p. 42)

"[...] familiarity with the conceptual codes that underlie a specific culture with its major artistic and normative manifestations."(de Graaf, de Graaf, \& Kraaykamp, 2000, p. 93)

"[...] familiarity with the dominant culture in a society, and especially the ability to understand and use 'educated' language."(A. Sullivan, 2001, p. 893, 2002, p. 145)

"[...] 'linguistic and cultural competence' and a broad knowledge of culture that belongs to members of the upper classes"(Dumais, 2002, p. 44)

"knowledge of, and to participate in, high culture such as classical music, theatre and museum"(S. Y. Cheung \& Andersen, 2003, p. 414)

"ability to conform to institutionalized expectations of educators" including "technical skills, including academic skills" (Lareau \& Weininger, 2003, p. 588)

"[...] knowledge of the dominant conceptual and normative codes inscribed in a culture." (Jæger, 2011, p. 283) 
Farkas et al. (1990, p. 127) defined cultural capital as "more general skills, habits, and styles" that are rewarded by teachers due to informal academic standards. This could be explained as the students' capabilities to comply with informal measures or norms in comparative evaluations by educators in terms of skills, ways of behaving and the physical appearance (habits and styles; compare above). Furthermore, the authors argue that these measures and norms do not relate to high culture.

Lareau and Horvat (1999, p. 42) suggest a similar definition of cultural capital:

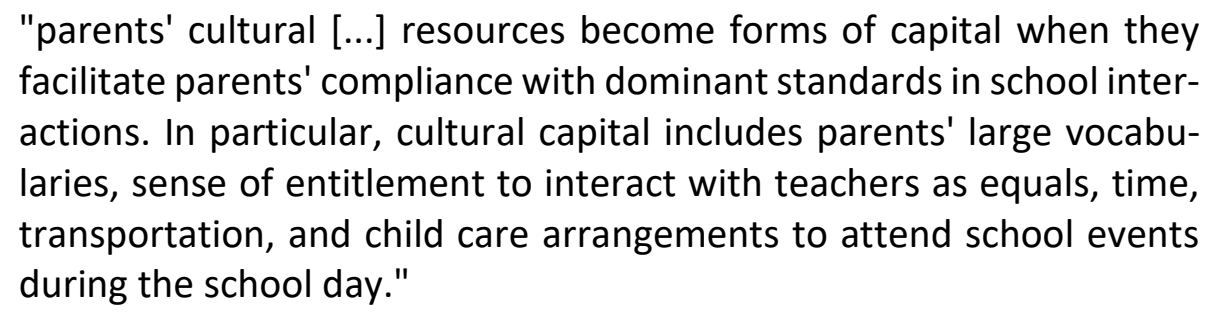

In other words, cultural capital is defined as parents' capabilities to comply with measures and norms in comparative evaluations by educators in the school context. Whereas Farkas et al. (1990) focus on the students' capabilities to comply with informal measures and norms in comparative evaluations in the school context, Lareau and Horvat (1999) emphasize parents' capabilities (Farkas et al., 1990; Lareau \& Horvat, 1999). Lareau and Horvat specify these capabilities as large vocabularies, time, transportation, childcare arrangements, and sense of entitlement. The latter concept is explained by Lareau as individuals' capability to question authorities, for example teachers, and interact with them as equals (Lareau, 2011, p. 2), in order to "pursue their own individual preferences and to actively manage interactions in institutional settings" (Lareau, 2011, p. 6). The concept of sense of entitlement could be explained as the belief to have the right to pursue one's own preferences, the confidence in one's ability to pursue those preferences and to handle interactions with authorities.

Weininger and Lareau (2003, p. 588) define cultural capital as students' and their parents' "ability to conform to institutionalized expectations of educators" including "technical skills, including academic skills". Regarding the expectations of educators Lareau and Weininger (2003, p. 588) referred to the criteria of evaluation (Bourdieu, 1977a, 
p. 495) in the educational system. Hence, the definition of cultural capital can be interpreted in terms of the capability of students and their parents to comply with established measures and norms in comparative evaluation by educators. Moreover, Lareau and Weininger (2003, p. 588) stressed that these criteria among others include skills. Thus, this notion of cultural capital comprises skills that are subject of the evaluation of students and parents by educators.

Another definition of cultural capital is proposed by Aschaffenburg and Maas (1997, p. 573): "proficiency in and familiarity with dominant cultural codes and practices - for example, linguistic styles, aesthetic preferences, styles of interaction." In other words, skills (proficiency) in and knowledge of (familiarity) the system of words, signs, or symbols representing cultural messages (code, compare chapter 2.1.1) and behavior (practices) that are defined as valuable (dominant). The meaning of "cultural" respectively "culture" in this context is not clear. Either it could refer to high culture, that is arts, music, theater, literature, etc. or to culture in general, that is anything that is created by humans.

De Graaf et al. (2000, p. 93) defined cultural capital as "[...] familiarity with the conceptual codes that underlie a specific culture with its major artistic and normative manifestations." A possible explanation is: Knowledge (familiarity) of the words, signs, or symbols used to represent ideas (conceptual codes) that are the basis of high cultural products (major artistic manifestations) and norms that are defined as valuable (major normative manifestations) in a particular group or society (specific culture).

Following Bourdieu (1977a, p. 494), Sullivan defines cultural capital as "[...] familiarity with the dominant culture in a society, and especially the ability to understand and use 'educated' language."(A. Sullivan, 2001, p. 893, 2002, p. 145). Hence, Sullivan's notion of cultural capital refers to knowledge of the culture and the linguistic skills that are defined as valuable in a society (dominant). It can be assumed that the concept of culture refers to high culture, since Sullivan explicitly refers to the concept of dominant culture (Bourdieu, 1977a), which is described as high culture. 
Dumais (2002, p. 44) defined cultural capital as "[...] 'linguistic and cultural competence' and a broad knowledge of culture that belongs to members of the upper classes". This definition can be explained as follows: linguistic and other skills as well as the knowledge that are needed to comprehend cultural products (see above), and the knowledge of culture that is characteristic to members of the upper classes. As above, it is not clear what the term culture means in this context. It can be interpreted as reference to high culture, that is, arts, music, theater, literature, and so forth or as reference to culture in general. To avoid the risk of excluding important aspects, culture can be interpreted as culture in general.

Jæger (2011, p. 283) termed "[...] knowledge of the dominant conceptual and normative codes inscribed in a culture" as cultural capital. This can be interpreted as knowledge of the words, signs, or symbols used to represent ideas (conceptual codes) and the sets of rules governing behavior (normative codes) of a particular group or society (culture).

\subsubsection{Definition of habitus}

According to Bourdieu, habitus links the social structure to action (Bourdieu, 1990; H.P. Müller, 2014, p. 37; Münch, 2004, p. 425; Swartz, 1997, p. 101). Social classes, characterized primarily by the distribution of economic and cultural capital (Bourdieu, 1984), imply distinct conditions of existence that form a scope of "possibilities and impossibilities, freedoms and necessities, opportunities and prohibitions" (Bourdieu, 1990, p. 54) for the individual members of a social class. Socialized within social class context, individuals unconsciously internalize (Swartz, 1997, p. 104) these aspects relevant to behavior (Bourdieu, 1987, p. 175, 1990, p. 54). The resulting dispositions or tendencies Bourdieu calls habitus. Since the habitus is the basis for behavior, the behavior of individuals of the same social class are similar (Bourdieu, 1987, p. 175). As Münch (2004, p. 425) states, habitus makes an analytical social class a social phenomenon. Moreover, Bourdieu argues that the entirety of individual behavior reproduces the social class structure. To denote that Bourdieu uses the term practice instead of behavior or action (Bourdieu, 1977b). 
To consider a wider array of definitions, Table 2 shows a selection of definitions of the term habitus. Besides different definitions from Bourdieu's work, some definitions from significant theoretical and empirical studies are selected.

Table 2: Definitions of habitus

"[...] a system of (partially or totally identical) schemes of perception, thought, appreciation and action" (Bourdieu \& Passeron, 1977, p. 35).

"The habitus is not only a structuring structure, which organizes practices and the perception of practices, but also a structured structure: the principle of division into logical classes which organizes the perception of the social world itself the product of internalization of the division into social classes." (Bourdieu, 1984, p. 170)

"[...] habitus, systems of durable, transposable dispositions, structured structures predisposed to function as structuring structures, that is, as principles which generate and organize practices and representations that can be objectively adapted to their outcomes without presupposing a conscious aiming at ends or an express mastery of the operations necessary in order to attain them." (Bourdieu, 1990, p. 53)

"Habitus in Bourdieu's work refers to a system of embodied dispositions which generate practice in accordance with the structural principles of the social world. Bourdieu's structuralist approach to the study of culture, his anthropology, captures in the concept of habitus the formal and informal mores, customs, or rules of a society and the unelaborated relations of homology which give access to another level of order and meaning." (Nash, 1990, pp. 432-433)

"Habitus is one's disposition, which influences the actions that one takes; it can even be manifested in one's physical demeanor, such as the way one carries oneself or walks. It is generated by one's place in the social structure; by internalizing the social structure and one's place in it, one comes to determine what is possible and what is not possible for one's life and develops aspirations and practices accordingly." (Dumais, 2002, p. 46)

"habitus is a set of attitudes and values" (A. Sullivan, 2002, p. 149)

"Habitus is the set of learned preferences or dispositions (styles of thought and action) by which a person orients to the social world. It is the internalized interpretive framework through which one perceives the social world and one's place within it, and correspondingly calibrates one's aspirations and appreciation of related actions/practice." (Edgerton, Roberts, \& Peter, 2013, p. 305)

Similar to the definitions of cultural capital, the definitions of habitus are oftentimes unclear. Hence, I will clarify the notion by interpretation and explication in the following. 
Bourdieu defined habitus as "[...] a system of (partially or totally identical) schemes of perception, thought, appreciation and action" (1977, p. 35). This could be explained as an interrelated set (system of (partially or totally identical) schemes) of beliefs about appearances (perception), thought, positive or negative evaluations of objects (appreciation), and action.

In his later work Bourdieu (1984, p. 170) defined habitus as follows:

"The habitus is not only a structuring structure, which organizes practices and the perception of practices, but also a structured structure: the principle of division into logical classes which organizes the perception of the social world itself the product of internalization of the division into social classes."

In other words, this definition can be paraphrased as an interrelated set (structure) of action (practices) and beliefs about certain actions (perception of practices) that is acquired by class-specific socialization (structured structure).

Moreover, Bourdieu (1990, p. 53) wrote:

"[...] habitus, systems of durable, transposable dispositions, structured structures predisposed to function as structuring structures, that is, as principles which generate and organize practices and representations that can be objectively adapted to their outcomes without presupposing a conscious aiming at ends or an express mastery of the operations necessary in order to attain them." (Bourdieu, 1990, p. 53)

Essentially, this definition can be summarized as an interrelated set (system) of positive or negative evaluations of performing a certain behavior (dispositions, compare chapter 2.1.1) that is acquired by socialization (structured structures; see above).

Nash (1990, pp. 432-433) explained Bourdieu's concept of habitus in the following way:

"Habitus in Bourdieu's work refers to a system of embodied dispositions which generate practice in accordance with the structural principles of the social world. Bourdieu's structuralist approach to the study of culture, his anthropology, captures in the concept of habitus the formal and informal mores, customs, or rules of a society and the 
unelaborated relations of homology which give access to another level of order and meaning."

This definition can be condensed to an interrelated set (system) of positive or negative evaluations of performing a certain behavior (embodied dispositions which generate practice) and the internalized shared rules governing behavior of a group of people (formal and informal mores, customs, or rules of a society).

Furthermore, Dumais (2002, p. 46) refers to habitus as

"[...] one's disposition, which influences the actions that one takes; it can even be manifested in one's physical demeanor, such as the way one carries oneself or walks. It is generated by one's place in the social structure; by internalizing the social structure and one's place in it, one comes to determine what is possible and what is not possible for one's life and develops aspirations and practices accordingly."

This definition can be explained both, as positive or negative evaluations of performing a certain behavior (disposition), including body language, for example posture, way of walking (physical demeanor, such as the way one carries oneself or walks), aspirations, and behavior (practices), that are acquired by class-specific socialization (generated by one's place in the social structure; by internalizing the social structure).

Sullivan (2002, p. 149) defined habitus as "a set of attitudes and values". Attitudes can be referred to as positive or negative evaluations of objects or behavior. Furthermore, values can be conceived as internalized shared rules governing behavior of a group of people.

According to the definition of Edgerton et al. (2013, p. 305),

"Habitus is the set of learned preferences or dispositions (styles of thought and action) by which a person orients to the social world. It is the internalized interpretive framework through which one perceives the social world and one's place within it, and correspondingly calibrates one's aspirations and appreciation of related actions/practice." 
Thus, habitus might refer to a set of positive or negative evaluations of objects (preferences), thought (styles of thought), positive or negative evaluations of performing a certain behavior (dispositions, appreciation of related actions/practice), and aspirations.

\subsubsection{Disentangling cultural capital, habitus, and practice}

In the previous sections, the definitions of the theoretically central terms cultural capital and habitus have been explored. The aim of this chapter is to define cultural capital and habitus in coherent, disjoint, and complementary ways for the use in this thesis. Table 3 gives a systematic overview of the definitions of cultural capital and habitus that are derived in chapters 2.1.1 and 2.1.2. For reasons of clarity and comprehensibility, the definientia are arranged by categories that are denoted in the first column. These categories are formed as generic terms of the definientia. The second column contains the definientia, that is, the terms that are used to explain the terms cultural capital and habitus. The third and fourth column indicate to which of the definienda, that is the terms to be explained, the respective definiens refers: cultural capital, habitus, or to both. In the last column, the references are listed.

Table 3 shows that both terms cultural capital and habitus are defined in diverse ways. This is especially true for cultural capital as the definientia can be assigned to eight categories: general characteristics of a social class, subjective evaluations, beliefs and cognitions, skills, knowledge, behavior and action, and individual appearance to organizational resources. In sum, 22 different definitions of cultural capital can be identified. Definitions of habitus belong to five categories: subjective evaluations, norms and values, beliefs and cognition, behavior and action, and individual appearance. In total, eight definitions of habitus can be found. Moreover, the following four definientia are used to define cultural capital and habitus: positive or negative evaluation of performing a certain behavior, positive evaluations of certain objects, behavior respectively action, and body language. Thus, cultural capital and habitus have become umbrella terms including highly differing concepts and they partially overlap. 


\begin{tabular}{|c|c|c|c|c|}
\hline \multirow[b]{2}{*}{ Category } & \multirow[b]{2}{*}{ Definientia } & \multicolumn{2}{|c|}{ Definienda } & \multirow[b]{2}{*}{ Citation } \\
\hline & & $\begin{array}{l}\text { Cultural } \\
\text { Capital }\end{array}$ & Habitus & \\
\hline \multirow{2}{*}{$\begin{array}{l}\text { General } \\
\text { characteristics }\end{array}$} & characteristics that are typical only for the upper classes*4 & 1 & 0 & (DiMaggio, 1982) \\
\hline & $\begin{array}{l}\text { capability of students and their parents to comply with estab- } \\
\text { lished measures and norms in comparative evaluation by educa- } \\
\text { tors* }\end{array}$ & 1 & 0 & $\begin{array}{l}\text { (Farkas et al., 1990; Lareau \& Horvat, 1999; Lareau } \\
\text { \& Weininger, 2003) }\end{array}$ \\
\hline \multirow[t]{2}{*}{$\begin{array}{l}\text { Subjective eval- } \\
\text { uations }\end{array}$} & $\begin{array}{l}\text { (interrelated sets of) positive or negative evaluation of perform- } \\
\text { ing a certain behavior }\end{array}$ & 1 & 1 & $\begin{array}{l}\text { (Bourdieu, 1986; Dumais, 2002; Edgerton et al., } \\
\text { 2013; Nash, 1990; A. Sullivan, 2002) }\end{array}$ \\
\hline & (interrelated sets of) positive evaluations of certain objects & 1 & 1 & $\begin{array}{l}\text { (Bourdieu \& Passeron, 1977; DiMaggio, 1982; } \\
\text { Edgerton et al., 2013; A. Sullivan, 2002) }\end{array}$ \\
\hline \multirow[t]{2}{*}{$\begin{array}{l}\text { Norms and val- } \\
\text { ues }\end{array}$} & $\begin{array}{l}\text { interrelated sets of internalized shared rules governing behavior } \\
\text { of a group of people }\end{array}$ & 0 & 1 & (Nash, 1990; A. Sullivan, 2002) \\
\hline & aspirations & 0 & 1 & (Dumais, 2002; Edgerton et al., 2013) \\
\hline \multirow{4}{*}{$\begin{array}{l}\text { Beliefs and cog- } \\
\text { nitions }\end{array}$} & interrelated sets of thought* & 0 & 1 & (Bourdieu \& Passeron, 1977; Edgerton et al., 2013) \\
\hline & interrelated sets of beliefs about appearances* & 0 & 1 & (Bourdieu, 1984; Bourdieu \& Passeron, 1977) \\
\hline & confidence in one's own knowledge & 1 & 0 & (Bourdieu \& Passeron, 1977) \\
\hline & $\begin{array}{l}\text { belief to have the right to pursue one's own preferences, the } \\
\text { confidence in one's ability to pursue those preferences and in in- } \\
\text { teractions with authorities** }\end{array}$ & 1 & 0 & (Lareau \& Horvat, 1999) \\
\hline
\end{tabular}

\footnotetext{
${ }^{4}$ For an explanation, see text below
} 


\begin{tabular}{|c|c|c|c|c|}
\hline \multirow[b]{2}{*}{ Category } & \multirow[b]{2}{*}{ Definientia } & \multicolumn{2}{|c|}{ Definienda } & \multirow[b]{2}{*}{ Citation } \\
\hline & & $\begin{array}{l}\text { Cultural } \\
\text { Capital }\end{array}$ & Habitus & \\
\hline \multirow[t]{6}{*}{ Skills } & skills & 1 & 0 & (Farkas et al., 1990; Lareau \& Weininger, 2003) \\
\hline & cognitive and linguistic skills & 1 & 0 & (Bourdieu, 1977a, 1986) \\
\hline & linguistic skills & 1 & 0 & (A. Sullivan, 2001) \\
\hline & linguistic skills that are needed to comprehend cultural products & 1 & 0 & (Dumais, 2002) \\
\hline & large vocabularies & 1 & 0 & (Lareau \& Horvat, 1999) \\
\hline & skills that are needed to comprehend cultural products & 1 & 0 & (Aschaffenburg \& Maas, 1997; Dumais, 2002) \\
\hline \multirow[t]{4}{*}{ Knowledge } & knowledge & 1 & 0 & (Bourdieu, 1977a, 1986) \\
\hline & knowledge of culture that is characteristic for the upper classes & 1 & 0 & (Dumais, 2002; A. Sullivan, 2001) \\
\hline & knowledge that is needed to comprehend cultural products & 1 & 0 & $\begin{array}{l}\text { (Aschaffenburg \& Maas, 1997; de Graaf et al., } \\
\text { 2000; Dumais, 2002; Jæger, 2011) }\end{array}$ \\
\hline & knowledge of the sets of shared rules governing behavior** & 1 & 0 & (de Graaf et al., 2000; Jæger, 2011) \\
\hline $\begin{array}{l}\text { Behavior and ac- } \\
\text { tion }\end{array}$ & (interrelated sets of) behavior, action & 1 & 1 & $\begin{array}{l}\text { (Aschaffenburg \& Maas, 1997; Bourdieu, 1984; } \\
\text { Bourdieu \& Passeron, 1977; DiMaggio, 1982; Du- } \\
\text { mais, 2002; Farkas et al., 1990) }\end{array}$ \\
\hline \multirow{2}{*}{$\begin{array}{l}\text { Individual } \\
\text { appearance }\end{array}$} & physical appearance $* * *$ & 1 & 0 & (DiMaggio, 1982; Farkas et al., 1990) \\
\hline & $\begin{array}{l}\text { body language, i.e. posture, way of walking, gestures, and facial } \\
\text { expressions*** }\end{array}$ & 1 & 1 & (Bourdieu, 1986; Dumais, 2002) \\
\hline \multirow{3}{*}{$\begin{array}{l}\text { Organizational } \\
\text { resources }\end{array}$} & time** & 1 & 0 & (Lareau \& Horvat, 1999) \\
\hline & transportation** & 1 & 0 & (Lareau \& Horvat, 1999) \\
\hline & child care arrangements** & 1 & 0 & (Lareau \& Horvat, 1999) \\
\hline
\end{tabular}


This unclarity leads to major methodological problems (Opp, 2005, p. 134): It complicates the scientific discussion about the role of cultural capital and habitus in educational inequality. Furthermore, it makes it difficult to criticize and empirically test the theories. In the end, unclear terminologies make the development of theories difficult.

To avoid problems related to an unclear terminology, central concepts considered in this thesis are defined and assigned to the definienda cultural capital and habitus in the following. Table 3 serves as a basis for this procedure. The aim is to obtain a clear, coherent, disjoint, and complementary conceptual basis preserving the explanatory potential of the theories regarding educational inequality.

Some of the definientia of cultural capital and habitus in Table 3 are not considered for defining concepts used in the current thesis. As already mentioned in chapter 2.1, including all definientia is no requirement for formulating a useful theory. Definientia are discarded for the following reasons: ambiguity $\left({ }^{*}\right)$, non-coherence with the theoretical focus of the thesis as a whole $(* *)$, lack of empirical relevance $(* * *)$.

The remaining definientia are assigned to the definienda cultural capital and habitus. As already mentioned, the respective definitions should be disjoint and complementary. Furthermore, the definientia assigned to these definienda should be substantially coherent with the entirety of the assigned definientia.

Regarding cultural capital, it seems plausible to designate definientia that represent ability, that is, skills and knowledge. These characteristics are suitable to formulate hypotheses with a high explanatory power. Moreover, they are uniquely used to define cultural capital not habitus. Therefore, cultural capital is specified as cognitive and linguistic skills and knowledge. These definientia subsume linguistic skills that are needed to comprehend cultural products, large vocabularies, skills that are needed to comprehend cultural products, knowledge of culture that is characteristic for the upper classes, and knowledge that is needed to comprehend cultural products. 
In a more general perspective, habitus can be described as characteristics of the mind that are related to behavior and action. Table 3 shows that these kinds of definientia of habitus are predominant. Following that reasoning, habitus is defined as internalized characteristics that guide action and behavior, that is attitudes, norms and values, idealistic aspirations and beliefs about one's own skills and knowledge. Attitudes refer to positive or negative evaluations of performing certain behaviors (compare Ajzen, 1988, p. 117) or of certain objects. Norms are defined as internalized shared rules governing behavior of a group of people. Values refer to internalized shared general principles guiding behavior of a group of people. Hence, norms are derived from values. Idealistic aspirations are defined as normative expectations regarding goal-directed behavior (compare Haller, 1968, p. 484; Stocké, Blossfeld, Hoenig, \& Sixt, 2011, p. 107). Psychological research shows that beliefs about one's own skills and knowledge are crucial for the academic engagement (Bong \& Skaalvik, 2003, pp.6-7see chapter 2.3.1). Hence, this definiens instead of confidence in one's own knowledge is used because skills seem to be crucial complements of knowledge in this context.

The remaining definiens (interrelated sets of) behavior or action are considered as a notion of its own as assigning it to cultural capital or habitus would impair the coherence of these terms. Behavior and action are substantially different from ability and internalized characteristics guiding behavior. Hence, practice is defined as action and behavior determined by habitus.

After defining the central concepts of the theory, the state of research considering mechanisms leading to educational inequality will be described in the following chapters.

\subsection{Habitus: State of research}

In the following, the state of research regarding the acquisition and the impact of habitus on academic achievement is presented. Here, the term habitus is used in the sense of the definition in chapter 2.1.3. 
According to this definition, habitus comprises attitudes, norms and values, idealistic aspirations and beliefs about one's own skills and knowledge. These characteristics guide action and behavior and they are shaped by the individual social class position. The behavioral manifestation of habitus is termed practice. From this point of view, the factors that are commonly applied to explain educational inequalities in quantitative research, that is high cultural participation and reading activities, refer to practices resulting from habitus. Furthermore, some recent studies on educational inequalities seize the notion of habitus and differentiate it from the common concept of cultural capital (Dumais, 2002; Edgerton et al., 2013; Gaddis, 2013; Grgic \& Bayer, 2015; Wildhagen, 2009). Habitus is operationalized in various ways: occupational aspirations (Dumais, 2002), realistic educational aspirations (Edgerton et al., 2013; Wildhagen, 2009), idealistic educational aspirations (Grgic \& Bayer, 2015), attitudes towards teachers (Edgerton et al., 2013), academic self-concept (Gaddis, 2013; Grgic \& Bayer, 2015), self-esteem (Grgic \& Bayer, 2015), and attitudes towards education (Edgerton et al., 2013; Gaddis, 2013).

\subsubsection{Acquisition of habitus}

As mentioned in chapter 2.1.2, habitus is the product of socialization (Bourdieu, 1984, 474, 475; see also Goldthorpe, 2007, pp.82-83; Nash, 1990, p. 434; Swartz, 1997, p. 104). This is consistent with socialization research. Socialization is a process through which, in mutual interdependence between the biopsychic basic structure of individual actors and their social and physical environment, relatively durable dispositions of perception, evaluation and action arise on an individual as well as on a collective level (Hurrelmann, 2008, p. 25). On the other hand, habitus is defined as internalized characteristics that guide action and behavior (see chapter 2.1.3). It can be concluded that socialization comprises the development of habitus.

Basically, it is argued that habitus is shaped by the objective conditions of existence (Bourdieu, 1984, p. 101, 1990; H.-P. Müller, 2014, p. 37; Münch, 2004, p. 425; Swartz, 
1997, p. 101), that is the familial endowment with capital. ${ }^{5}$ By definition, the endowment with capital determines the social class position. Thus, habitus is class-specific. As Münch (2004, p. 422) puts it, habitus is embodied social structure. Habitus consists of certain dispositions to act and a view of the social world and one's place within it (Dumais, 2002, p. 46; Edgerton et al., 2013, p. 305; Münch, 2004, p. 422). Individuals develop a behavioral orientation towards the possible avoiding the impossible based on past experiences (Bourdieu, 1990, p. 54). These opportunities and restrictions are closely related with the capital available to individuals.

For example, according to Münch (2004, p. 423), a member of the working-class has a limited prospect. His aspirations are restricted to his own social sphere and limited regarding time horizon. He is frugal, has a low tendency towards social mobility, shows respect for people from higher social classes, and has low self-esteem considering unfamiliar activities. In contrast, the habitus of a member of the lower middle class (for example craftsmen, merchants, low officials, nurses, social worker, host, bodybuilder) is characterized by formalism (work, home, leisure), distinction from the working class (living, food, dress, manners, leisure activities, social association, cultural consumption), lack of self-confidence, and awareness of the own limits. Aspiring towards upward social mobility, parents invest in the education of their offspring of whom successful upward mobility is expected (Münch, 2004, p. 424).

However, these descriptions are ideal types of habitus that can be referred to as class habitus. Nash (1990, p. 434) calls these ideal types collective habitus. The class habitus can be distinguished from the individually internalized habitus (compare Nash, 1990, p. 434) that represents a variation of the class habitus as it is a result of individual socialization. In the following, socialization as the process of the acquisition of habitus is described.

\footnotetext{
${ }^{5}$ That is economic capital, cultural capital, and social capital (for definitions of economic and social capital see Bourdieu, 1986).
} 
According to Bourdieu, habitus is primarily acquired within the family of origin by early socialization (Bourdieu \& Passeron, 1971, 44, 86, 115, 1977, p. 42; Swartz, 1997, 103, 104). Although he admits that the educational system also has a formative influence on habitus, changes of habitus are always based on previous dispositions. Primary socialization "produces a primary habitus, characteristic of a group or class which is the basis for the subsequent formation of any other habitus" (Bourdieu \& Passeron, 1977, p. 42). New socialization experiences are more likely to be integrated into the existing dispositional system and to elaborate it rather than to change habitus fundamentally (H.-P. Müller, 2014, p. 99; Swartz, 1997, p. 107). Furthermore, Bourdieu assumes that the pedagogic work of the educational system tends to reinforce the class-specific habitus (Münch, 2004, p. 425). The educational system imparts a specific habitus, that is, the class habitus of the social class possessing the most cultural capital. This habitus could be called school habitus. The efficacy of this pedagogic work depends on the distance of the individual habitus and the school habitus: the bigger the distance is the lower is the efficacy of the pedagogic communication of school habitus (Bourdieu \& Passeron, 1977, $43,51)$. Summarized, from Bourdieu's perspective, habitus is formed mainly in the process of early socialization when the primary habitus is produced. Building on the primary habitus, there is potential for changes of habitus over the course of lifetime but these changes are rather limited.

\section{Attitudes towards culture}

In the field of educational sociology, cultural capital is commonly measured by surveying the frequency of certain cultural activities, for example going to the theater, the opera, or art exhibitions. As argued above, cultural activities are specific actions respectively practices, that is the manifestation of habitus. Thus, cultural practices are proxy measurements for habitus, in terms of attitudes towards high culture. There are several studies investigating the relation of social class and cultural practices (de Graaf et al., 2000; Dumais, 2002; Rössel \& Beckert-Zieglschmid, 2002; A. Sullivan, 2001). Using data from the Netherlands Family Survey (1992-1993) De Graaf et al. (2000, pp. 102-103) show that high cultural practices are correlated with parents' education. Dumais (2002, p. 52) 
reveals that participation in cultural lessons and high cultural practices of US eighth graders are associated with their socioeconomic background. For the analysis, she draws on data from the US NELS study ${ }^{6}$ from 1988. Surveying English eleventh grade students, Sullivan (2001, p. 900) shows that parental cultural practices are related to social class and education. Cultural practices are measured by a compound index including participation in formal culture, reading, number of books in the home, newspapers taken, type of music and radio stations listened to, subjects discussed by parents at home (A. Sullivan, 2001, p. 900). Rössel and Beckert-Zieglschmid (2002, p. 506) show that high cultural practices are associated with social class ${ }^{7}$, parental education and social situation ${ }^{8}$. Members of the service class participated more often in high cultural practices than members of the other social classes. Furthermore, a better social situation and a higher education is linked to a higher participation in high cultural practices. The study of Mudiappa (2014, p. 92) showed that cultural practices of families with kindergartners (visiting a museum, cinema, concert, theater, library) is related to parents' socioeconomic status (SES) and education. Parents with a higher SES and a higher education participate more often in high cultural practices with their kindergartners than parents with lower SES and lower education. Summarized, across the given countries, participation in cultural practices is related to social class and education. Thus, it can be concluded that positive attitudes towards high culture prevail in families with higher education and higher social class position. On the one hand, these results confirm the theoretical assumption of class-specific attitudes towards high culture. On the other hand, they are disregarding the mechanisms of the transmission of attitudes. However, some studies investigate these mechanisms in more detail.

\footnotetext{
${ }^{6}$ National Education Longitudinal Study

${ }^{7}$ Erikson-Goldthorpe-Portocarero class scheme (EGP)

${ }^{8}$ The index of the social situation comprises number of holiday trips within the last 12 months, number of cars, housing situation (own room, apartment, home ownership), and unemployment of the head of household.
} 
Mudiappa $(2014,55-56,92)$ shows that parents with an educated habitus participate more often in cultural practices with their kindergartners than parents with a less educated habitus. Introducing measurements of habitus, that is, idealistic educational aspiration, reading behavior, and the frequency of reading to their children into the model the relations of social class and parental education decrease and become insignificant. Furthermore, he demonstrates that the possession of cultural goods, that is artwork, books with poems, and classical literature (Mudiappa, 2014, p. 72), correlate with cultural practices with kindergartners (Mudiappa, 2014, p. 92). He suggests that the possession of these cultural goods corresponds with an educated habitus of the parents as it expresses familiarity with arts, a certain cultural taste, and a specific lifestyle (Mudiappa, 2014, p. 56). Overall, various indicators of habitus are associated with social class and parental education as well as with high cultural practices.

Sullivan (2001, pp. 900-902) reveals that the effect of parents' social class and education on students' cultural practices is entirely mediated by parental cultural practices. Students' cultural practices are operationalized as a composite measure of type and amount of books read, library use, newspapers read, type of television broadcasts watched, type of music listened to, playing an instrument, participation in formal culture (A. Sullivan, 2001, p. 899). Parental cultural practices comprised "reading (and number of books in the home), newspapers taken, type of music and radio stations listened to, participation in 'formal culture', and the subjects discussed by parents in the home" (A. Sullivan, 2001, p. 900). Thus, it can be concluded that attitudes towards these practices are passed on from parents to their children in the course of familial socialization.

Rössel and Beckert-Zieglschmid (2002) investigate the constitution of a high cultural orientation of eighth, ninth and tenth graders. Results imply that parents influence students' orientation as well as school context and peers (Rössel \& Beckert-Zieglschmid, 2002, pp. 507-508). Specifically, the parents' participation in high cultural practices and the proportion of classmates from families, which head of household had a university degree, have a positive impact on students' participation in high cultural practices. 
Attitudes towards language

According to Bourdieu (1977, p. 116), the acquisition of linguistic skills is always accompanied by the acquisition of specific attitudes towards language, that is a tendency towards a certain language use. Both linguistic skills and attitudes towards language are primarily learned in the family of origin. Thus, they are class-specific. Bourdieu distinguishes two ideal types of speech reflecting different attitudes towards language: bourgeois parlance and common parlance (Bourdieu \& Passeron, 1977, p. 115). These languages can be distinguished by various characteristics: pronunciation, speech melody, vocabulary, phraseology (Bourdieu \& Passeron, 1971, p. 112), correctness, accent, tone, rhythm of speech (Bourdieu \& Passeron, 1971, p. 114), and rhetorical ability (Bourdieu \& Passeron, 1977, p. 162). The bourgeois parlance is a sublime language characterized by a high proportion of lexical and syntactic borrowings from Latin (Bourdieu \& Passeron, 1977, p. 115), abstraction, formalism, intellectualism, and euphemistic moderation (Bourdieu \& Passeron, 1977, p. 116). In sum, these characteristics are primarily manifestations of a certain attitude towards the conversational partner and the object of conversation that can be described as distinguished distance, prudent ease, and contrived naturalness that reflect good manners (Bourdieu \& Passeron, 1971, p. 111, 1977, p. 116). In contrast, the common parlance of the working class is expressive, tends to mere illustrations of singular cases and to parables, banter, rudeness, ribaldry, and avoids "the bombast of fine words and the turgidity of grand emotions" (Bourdieu \& Passeron, 1971, p. 111, 1977, p. 116). The mastery of the bourgeois parlance cannot only be acquired within family but also has to be advanced within school resulting in a quasi-scholarly language use (Bourdieu \& Passeron, 1977, p. 115) and the corresponding attitudes towards language.

\section{Attitudes towards education and teachers}

Bourdieu states that attitudes towards education or schooling are determined by the probability of educational success (Bourdieu, 1977a, p. 495; Edgerton et al., 2013, p. 306). As cultural capital is the crucial factor of educational success and cultural capital 
is distributed unequally across the social classes, these attitudes towards education or schooling differ according to social class position. Individuals from families with high cultural capital have a high probability to attain favorable school leaving certificates and, thus, develop positive attitudes towards schooling. Consequently, students and their families with high cultural capital endowment and positive attitudes towards schooling tend to invest time, effort, and money necessary for educational success and, thus, conduct those practices that are rewarded at school. In contrast, students and their families with poor cultural capital tend to avoid investments in education and are less inclined to carry out rewarded practices. It has to be noted that the perceived probability of educational success is not based on rational estimations of individuals. According to the logic of habitus, it is rather grounded on past educational experiences of members of the same social class, especially the family, resulting in normative beliefs regarding the appropriate educational behavior. For the same reasons, attitudes towards teachers are depending on cultural capital, and hence, social class. Edgerton et al. (2013, p. 310) show that a composite measure of students' habitus consisting of attitudes towards post-secondary education and teachers as well as realistic educational aspirations correlate with the families' SES. In turn, the habitus index influences academic practices, that is, assignment completion, regular attendance, and task perseverance.

\section{Attitudes towards reading}

De Graaf et al. (de Graaf et al., 2000) show that parents' reading behavior is related to their education, on the one hand, and to the educational attainment of their children on the other hand. The authors argue that one theoretical explanation of the relation between parental reading behavior and students' educational attainment is that parents' reading behavior influences students' attitudes towards reading. Following this reasoning, students whose parents read often develop an affinity towards reading and read more frequently than students with negative attitudes towards reading.

Demir-Lira et al. (2019) conduct a longitudinal study on the effect of parents' early book reading to children. They survey shared book reading with children between one and 
two and a half years. They disclose that early shared book reading has a positive effect on the internal reading motivation in elementary school.

McElvany et al. (2008, p. 214) find that prior reading competence positively influences attitudes towards reading. That is, the better and more easily individuals understand what they read, the more they are motivated to read in the future.

\section{Educational Aspirations}

Several studies have pointed out class-specific educational achievement attitudes, norms, and values (for example Hyman, 1966; Paulus \& Blossfeld, 2007). A prominent concept in this field of research is educational aspiration. From a social psychological perspective, aspiration is "the cognitive orientational aspect of goal-directed behavior" (Haller, 1968, p. 484). Generally, educational aspiration refers to the level of education individuals strive to attain. In this respect, there is a substantial differentiation between idealistic and realistic aspirations (Haller, 1968, p. 484; Stocké et al., 2011, p. 107). Realistic aspirations represent the level of education that individuals believe they can actually achieve. Idealistic aspirations, however, are the normative expectations regarding the educational level.

The classical study of Sewell et al. (Sewell, Haller, \& Portes, 1969) operationalizes educational aspirations in terms of college plans. Hence, it is not determinable whether realistic or idealistic aspirations are measured. The same is true for the study of McDill and Coleman (1965). However, Sewell et al. (Sewell et al., 1969) show that educational aspirations are influenced by significant others, that is parents, teachers and peers. The parents' and teachers' encouragement to go to college as well as friends' college plans exerted a normative pressure on students to do so (Sewell et al., 1969, p. 87). Furthermore, McDill and Coleman $(1965,117,120,122)$ demonstrate that high school students' college aspiration is influenced by parents' education, parental desire that their offspring attends college, and by fellow students. Moreover, the impact of parents is higher than the fellow students' influence at high school entry. At the end of high school, the 
fellow students' impact is approximately as high as the parents' effect (McDill \& Coleman, 1965, p. 122). Thus, the influence of fellow students for educational aspirations increases in the course of secondary school while the parental impact decreases. Additionally, the scope of the fellow students' effect is greater for boys than for girls (McDill \& Coleman, 1965, p. 119).

Using NEPS data, Grgic and Bayer (2015) show that the probability of having high idealistic educational aspirations of both parents and students are related to social class and the educational experiences of their siblings (Grgic \& Bayer, 2015, p. 183). More specifically, aspirations are higher if students have older siblings that are experienced in upper secondary education. In addition, a composite measure of habitus is created. It consists of the students' academic self-concept, general self-esteem, and the idealistic educational aspiration. Besides social class and the educational experiences of their siblings, habitus is related to parental education, migration background, participation in high cultural practices, reading, tutoring, and parental support regarding school matters (Grgic \& Bayer, 2015, p. 185). Based on BiKS ${ }^{9}$ data, Paulus and Blossfeld (2007) investigate determinants of both idealistic and realistic educational aspirations of parents. Descriptive results suggest that parental idealistic aspirations are related to social class, education, and child's academic achievement. Parents tend to higher aspirations if they are members of higher social classes, hold higher educational certificates, and if their child has better grades (Paulus \& Blossfeld, 2007, pp. 499-501). Thus, parents seem to align their educational aspirations for their children according to their chances of success. Furthermore, the effect of grades seems to vary according to social class. Low class parents of middle and low achieving students reduce their idealistic aspirations more than middle and high class parents (Paulus \& Blossfeld, 2007, pp. 501-502).

Just as idealistic aspirations, parents' realistic educational aspirations are associated with social class, education, and child's academic achievement. Furthermore, realistic aspirations vary according to migration background and idealistic aspirations (Paulus

\footnotetext{
${ }^{9}$ Bildungsprozesse, Kompetenzentwicklung und Selektionsentscheidungen im Vor- und Grundschulalter (Educational processes, competence development and selection decisions in preschool- and school age)
} 
\& Blossfeld, 2007, pp. 504-505). Using the NELS data, Wildhagen (2009) shows that realistic educational aspirations are related to family income, parental education, sex, ethnicity, number of siblings, high cultural activities, time spent reading, mother's, father's, and friends' educational expectations, prior grades, and the academic track attended (Wildhagen, 2009, p. 186). Realistic educational aspirations are measured as a latent variable that entails years of education expected and college completion expected (Wildhagen, 2009, p. 182). Edgerton et al. (2013, p. 310) uses a composite measure of habitus comprising students' expected level of education, and indices measuring the students' disposition towards teachers, and their disposition towards post-secondary education. The habitus index is associated with the socioeconomic background, sex, and an interaction term of sex and the mean socioeconomic background at school (Edgerton et al., 2013, p. 313). Fend et al. (1973, p. 900) find that realistic educational aspirations are affected by parental aspirations and type of school. Parental aspirations are positively related to students' aspirations. Educational aspirations increase with the school track attended (Hauptschule, Realschule, Gymnasium) but are highest at comprehensive schools (Integrierte Gesamtschule) when academic achievement and social class are controlled. Moreover, Bandura et al. (1996, p. 1215) show that educational aspirations are determined by academic self-efficacy, that is the individual's beliefs in his or her abilities to achieve a certain academic goal (Bandura et al., 1996, p. 1206). ${ }^{10}$ Hence, students adapt their educational goals to their believed capabilities to reach these goals. On the other hand, parents align the educational aspirations they have for their children according to their academic efficacy, that is their beliefs in their abilities to further and support their children. However, parental academic efficacy is influenced by social class.

\footnotetext{
${ }^{10}$ The concepts of academic self-concept and academic self-efficacy are both formed by past achievement experiences and both determine academic engagement, goal-setting, task choice, persistence and effort, intrinsic motivation, strategy use, and achievement (Bong \& Skaalvik, 2003). However, there are substantial differences, for example, self-efficacy is goal-referenced and future-oriented whereas selfconcept is normative and oriented to the past (see for a detailed comparison Bong \& Skaalvik, 2003). Bong and Skaalvik (2003, pp. 30-31) conclude that self-efficacy is an "active precursor" of self-concept.
} 
Academic self-concept

One aspect of habitus crucial for academic achievement is the academic self-concept. It "refers to individuals' knowledge and perceptions about themselves in achievement situations" (Bong \& Skaalvik, 2003, p. 6). On the one hand, academic self-concept is based on past achievement. On the other hand, the current academic self-concept strongly influences academic engagement, goal-setting, task choice, persistence and effort, intrinsic motivation, strategy use, and achievement (Bong \& Skaalvik, 2003, pp. 6-7). Academic self-concept is formed by responses of the students' social context regarding their abilities. Guay, Marsh, and Boivin $(2003,125,132,133)$ show that it is determined by prior academic achievement and prior academic self-concept. Thus, in line with the skill-development model by Calsyn and Kenny (1977), achievement-related experiences in school form the academic self-concept. Additionally, the academic self-concept is shaped by comparison with reference groups, for example classmates (Bong \& Skaalvik, 2003, p. 3; Singer, 1981, 77, 79). As individuals have the need to assess themselves realistically, reference groups serve as standards of comparison. Thus, high achieving reference groups affect the individual's academic self-concept negatively, whereas low achieving reference groups have a positive effect (Stocké et al., 2011, p. 107). Moreover, the academic self-concept is formed by reflected appraisals from significant others (Bong \& Skaalvik, 2003, p. 3). Accordingly, Gniewosz et al. $(2015,468,469)$ find that the relation of academic achievement and students' academic self-concept is mediated by the parents' perception of their children's competencies.

\subsubsection{The impact of habitus on academic achievement}

Generally, the dimensions of habitus discussed in chapter 2.2.1 affect academic achievement by the following mechanisms: (1) elite status signals (attitudes towards high culture and high cultural practices), (2) motivation with regard to school-related learning (attitudes towards education and teachers, aspirations, and academic self-concept), and (3) motivation considering more general learning (attitudes towards reading). The effects of the motivational dimensions of habitus on academic achievement only occur if 
habitus is reflected in practice. A student highly motivated to learn for school as he aspires to Abitur (A level) receives good grades only if he or she actually does learn effectively. In the following, the mechanisms associated with habitus that influence academic achievement are described in more detail.

\section{Social distinction by elite status signals}

There are many studies that refer to the mechanism of elite status signals (Aschaffenburg \& Maas, 1997; de Graaf et al., 2000; DiMaggio, 1982; DiMaggio \& Mohr, 1985; Dumais, 2002; Gaddis, 2013; Jæger, 2011; Lamont \& Lareau, 1988; Lareau \& Weininger, 2003; A. Sullivan, 2001). In his early works, Bourdieu (1977a; 1971, 1977) stresses its importance for academic achievement. He assumes that there is a set of dispositions or attitudes that has a signal effect indicating the students' education. These are certain attitudes towards language, education, culture (Bourdieu \& Passeron, 1971, 112, 121), and cultural practices (Bourdieu, 1977a, p. 506). Students' attitudes influence the teachers' perception of students regarding their education independent of students' actual knowledge and skills. The manifestations of these attitudes, that is certain practices, serve as means of distinction from the uneducated masses. Hence, students showing positive attitudes towards language, education, culture and that participate in prestigious cultural activities are assessed better by teachers than students that do not exhibit these characteristics.

This applies particularly if students' assessment by teachers follow "implicit, diffuse criteria of the traditional art of grading", for example presentations or oral exams (Bourdieu \& Passeron, 1977, p. 162). In these situations teachers unconsciously tend to assess students by aspects of practice including their language use (Bourdieu \& Passeron, 1971, p. 114, 1977, p. 162). According to Bourdieu and Passeron (1977, p. 116), the language use is of outstanding importance. They argue that the educational system demands a certain attitude towards language that results in a particular language use. Within the educational system, the bourgeois language, that is the language of the educated clas- 
ses, is highly valued whereas the common parlance, that is the language of the uneducated working classes, is devalued. Students using the bourgeois language are perceived by teachers as educated and gifted (Bourdieu \& Passeron, 1971, p. 112). That in turn, they $(1971$, p. 115) argue, leads teachers to give these student better grades than students using a less distinguished language. High linguistic skills are a necessary precondition for the mastery of the bourgeois language (compare chapter 2.2.1) but it is not a sufficient one. Bourgeois language presupposes rich vocabulary, grammatical skills, the ability to think in abstract categories, correct pronunciation, et cetera. However, bourgeois language refers to a certain language use rather than linguistic skills.

Concerning elite status signals, DiMaggio's study (1982) is very influential (Lareau \& Weininger, 2003). Referring to Bourdieu's theory of cultural capital (Bourdieu, 1977a; Bourdieu \& Passeron, 1977) and Weber's work on status group culture (Weber, 1978), DiMaggio conceptualizes a cultural theory. Status groups evolve their own distinctive cultural traits, tastes, and styles. Status group culture serves to secure social boundaries and, thus, a coherent group composition. The final goal of status groups is to protect their access to scarce social, economic, and cultural resources, for example higher educational diplomas. Moreover, it is argued that in modern societies status group members do not necessarily know each other. Hence, status group membership is signaled via participation in status group culture. Thus, participation in high status culture becomes cultural capital and can be suitable to gain higher diplomas and better grades. In principal, students from any social class could participate in high status culture. This bears the potential for upward mobility. DiMaggio assumes that $(1982$, p. 190)

"teachers [...] communicate more easily with students who participate in elite status cultures, give them more attention and special assistance, and perceive them as more intelligent or gifted than students who lack cultural capital."

Elite status signals in terms of high cultural participation show significant effects on grades in English (in the US), German (in Germany), History, and mathematics (DiMaggio, 1982; Rössel \& Beckert-Zieglschmid, 2002). 


\section{Motivation}

Motivational aspects play a crucial role for the explanation of learning and school-related practices and, in turn, academic achievement. Studies examining the influence of habitus on educational outcomes operationalize habitus by educational aspirations either idealistic (Grgic \& Bayer, 2015) or realistic (Edgerton et al., 2013; Wildhagen, 2009), academic self-concept (Gaddis, 2013; Grgic \& Bayer, 2015), as well as attitudes towards education (Edgerton et al., 2013; Gaddis, 2013) and teachers (Edgerton et al., 2013). Furthermore, the study of de Graaf et al. (2000, pp. 107-108) suggests that attitudes towards reading represent an explanation of educational inequalities.

Considering educational aspirations, it is assumed that a high educational aspiration leads to a high motivation to reach these educational goals (Sewell et al., 1969, p. 83). Hence, students with high educational aspirations tend to more advantageous academic practices than students with low educational aspirations resulting in higher academic achievement.

In addition, the academic self-concept influences motivation (see also 'academic selfefficacy' Bandura et al., 1996, p. 1217; Gniewosz et al., 2015, p. 461; Guay \& Vallerand, 1996/1997, p. 225), and hence, academic achievement (Guay et al., 2003, pp. 133-134). Students with a positive academic self-concept have a higher motivation for advantageous academic practices because they enjoy these activities (Gniewosz et al., 2015, p. 461; Guay \& Vallerand, 1996/1997, p. 225). This finding is in accordance with the selfenhancement model (Calsyn \& Kenny, 1977) that academic self-concept causes academic achievement. As the academic self-concept is determined by prior academic achievement (see chapter 2.2.1), the causal relationship of academic self-concept and achievement can be described as a Matthew effect as coined by Merton (1968). High achieving students perceive themselves as being good at school that in turn motivates them to step up efforts and, in the end, do even better.

Moreover, attitudes towards education and teachers are motivating factors. Edgerton et al. $(2013$, p. 310$)$ show that these attitudes, in combination with realistic educational 
aspirations, are associated with academic practices. Moreover, they reveal that academic practices influence competencies in mathematics, reading, and science. Habitus is operationalized as a composite measure of students' expected level of education that can be interpreted as realistic educational aspirations, disposition toward teachers and disposition toward post-secondary education. Academic practices are operationalized as an index of several Likert scale items like "I complete my assignments.", "I complete my homework on time.", "When schoolwork is very difficult, I stop trying." (Edgerton et al., 2013, p. 311). In short, students with higher educational aspirations and positive attitudes towards teachers and higher education worked harder in school and reached higher skills in the domains mathematics, reading, and science. Farkas et al. (1990, p. 140) disclose that students with positive work habits have higher coursework mastery which in turn determines course grades.

Furthermore, reading plays a central role in school (de Graaf et al., 2000, p. 108) and the acquisition of linguistic skills and knowledge (A. Sullivan, 2001), and hence, for academic achievement. Accordingly, characteristics influencing reading practices should be considered. Baker et al. (1999, p. 470) show that various dimensions of attitudes towards reading influence reading behavior. The most relevant dimensions for explaining reading behavior refers to intrinsic motivation. This can be interpreted in accordance with the concept of habitus as dispositions that determine behavior. Based on panel data, McElvany et al. (2008, p. 214) demonstrate that prior attitudes towards reading affect reading practice and prior reading practice influences reading competence. Moreover, De Graaf et al. (2000, p. 108) suggest that, besides skills related to reading, the attitudes towards reading should be taken into account. Since reading is common in school, students with a positive attitude towards reading have an advantage over students with negative attitudes towards reading. More specifically, it is argued that the latter experience a cultural shock, as they are not used to read.

Overall, aspiring for high educational attainment, believing in one's own academic abilities, and having positive attitudes towards education and teachers increases the indi- 
vidual's academic motivation, efforts, and ultimately academic achievement. Furthermore, students with positive attitudes towards reading have a higher motivation considering (1) schoolwork related to reading and (2) extracurricular reading. As they read more frequently, they acquire more linguistic skills and knowledge. In contrast, students with negative attitudes towards reading avoid reading and perceive school as a strange and adverse context as reading is a practice central to school.

\subsection{Cultural capital: State of research}

In the following chapter, the state of research on cultural capital as defined in chapter 2.1.3 is portrayed. Chapter 2.3.1 deals with the acquisition of cultural capital. Thereafter chapter 2.3.2 on the impact of cultural capital on academic achievement follows.

As described in chapter 2.1.3, cultural capital is defined as cognitive and linguistic skills and knowledge. Regarding literature on the acquisition of cultural capital and its impact on academic achievement, some studies consider measures of (cultural) knowledge ${ }^{11}$ (Becker, 2010; A. Sullivan, 2001), ${ }^{12}$ cognitive skills (Farkas et al., 1990) ${ }^{13}$ or linguistic skills (A. Sullivan, 2001). However, these studies reveal that these dimensions of cultural capital are significantly associated with social class on the one hand and educational outcomes on the other hand. Further empirical studies show that across countries and different stages of life beginning at the age of three years cognitive and linguistic skills are related to social origin (Baumert \& Schümer, 2001; Linberg, Schneider, Waldfogel, \& Wang, 2019; Maehler et al., 2013; Weinert, Ebert, \& Dubowy, 2010; Wendt, Stubbe, \& Schwippert, 2012).

Chapter 2.3 focusses on linguistic skills and high cultural knowledge. Bourdieu emphasizes the role of linguistic skills for educational success (for example Bourdieu, 1977a;

\footnotetext{
${ }^{11}$ Cultural knowledge can be defined as "knowledge of those cultural products from literature, classical music, theatre, and visual arts that are commonly conceived as valuable in a society" (Goßmann \& Mätzke, 2019, p. 4).

12 DiMaggio (1982) considers "cultural information" as a measure of cultural capital that is equivalent to cultural knowledge but for statistical reasons, he excludes the variable from the empirical tests of his hypotheses.

${ }^{13}$ More specifically, basic skills are measured by the mean score of two subtests of the lowa Tests of Basic-Skills, language and mathematics (Farkas, Grobe, Sheehan, \& Shuan, 1990, p. 131).
} 
Bourdieu \& Passeron, 1977). Moreover, de Graaf et al. (2000) argue that reading affects educational success as it furthers linguistic skills. Some more recent studies support that hypothesis (Mikus et al., 2020; A. Sullivan, 2001). High cultural knowledge is taken into account as high culture is often considered as characteristic for cultural capital (Goldthorpe, 2007; Kingston, 2001; Lareau \& Weininger, 2003).

\subsubsection{Acquisition of cultural capital}

The subchapter begins with linguistic skills and then moves on to high cultural knowledge.

Linguistic skills

The analysis of Weinert et al. (2010) is based on a sample of 547 children in 97 day care centers in Bavaria and Hesse. It demonstrates that various linguistic skills of three year old of kindergartners differ by social origin. They find that, more specifically, linguistic competence and verbal memory varies by maternal vocational education: The higher mothers' education the higher the competencies. Social disparities are highest for linguistic competence. Linberg et al. (2019) investigate the differences in linguistic skills of about six to seven year old children in Germany and the US. Comparing children of highly educated parents with children of parents with low formal education, reveals differences in linguistic skills amounting to 0.85 standard deviations in the US case and to 1.24 standard deviations in the German case. The authors consider these gaps as quite substantial (Linberg et al., 2019, p. 16). The analysis is based on representative samples from both countries. Based on PIRLS data ${ }^{14}$, Wendt et al. (2012) show that reading competence of fourth graders in all the 56 participating educational systems is affected by social origin. In PIRLS data from Germany 2011, Wendt et al. (2012, p. 185) find differences between students from the highest EGP ${ }^{15}$-class (EGP I) and the lowest EGP-class (EGP VII) that correspond to 0.80 of a standard deviation. Using PISA data ${ }^{16}$, Baumert and Schümer (2001, pp. 381-385) reveal that linguistic skills (reading competence) of 15

\footnotetext{
${ }^{14}$ Progress in International Reading Literacy Study

${ }^{15}$ Erikson-Goldthorpe-Portocarero class scheme

${ }^{16}$ Program for International Student Assessment
} 
year old students differ by social origin measured as highest ISEI ${ }^{17}$ of the parents in all the 32 participating countries. In comparison to the other participating countries, these differences are highest in Germany (Baumert \& Schümer, 2001, p. 384). Comparing the mean reading competence of students from the upper quartile of the ISEI measure with the mean of those students from the lower quartile, social differences amount to 1.2 standard deviations (Baumert \& Schümer, 2001, p. 383). Social differences in linguistic skills persist until adulthood. Drawing on PIAAC data ${ }^{18}$, Maehler et al. (2013, pp. 112113) disclose that social origin measured as parental education affects reading competence of adults aged 16 to 65 . These differences amount up to 1.17 standard deviations in Germany (Maehler et al., 2013; Zabal et al., 2013). Studies on children's language development show that children with a higher SES have higher skills than children with a lower SES regarding vocabulary, grammar, communication of meaning through language and drawing meaning from language (Hoff, 2006). Furthermore, language use of students differs by SES (Hoff, 2006). Higher SES students use language more frequently to analyze and reflect, to reason and justify, and to predict and consider alternative possibilities than lower SES students.

However, how do these inequalities occur? According to Bourdieu, cultural capital is acquired by the work of transmission ${ }^{19}$ (Bourdieu, 1986, p. 246) in the families of origin, that is the familial socialization, and the work of acquisition (Bourdieu, 1986, p. 244), that is the time an individual spends on learning. However, he stresses that cultural capital is primarily acquired by familial socialization (Bourdieu, 1977a). According to this view, it is not taught in school. Moreover, Bourdieu argues that cultural capital is unequally distributed across social classes: Only the upper social classes possess the cultural capital that is required in school. Parents transmit it to their children intentionally (Bourdieu, 1977a, p. 495) or unintentionally in everyday life from the earliest age (Bourdieu,

\footnotetext{
${ }^{17}$ The International Socio-Economic Index of Occupational Status is based on international data on income and education of different professions ranging from 19 to 90.

${ }_{18}$ Program for the International Assessment of Adult Competencies

${ }^{19}$ Bourdieu also uses the terms educational work (for example Bourdieu, 1977a, p. 494) and pedagogic work (Bourdieu, 1977a, p. 502) of the families to refer to the acquisition of cultural capital by the process of familial socialization.
} 
1986, p. 246). Hence, only upper social class students are endowed with the cultural capital that is a precondition for positive assessments by teachers.

Lareau (2002) investigates whether social class differences in childrearing exist and how they can be described. Drawing on qualitative data of children aged between 8 and 10 from middle-class, working-class, and poor families, she concludes that social class differences in childrearing actually exist. Accordingly, middle-class parents show a specific childrearing pattern that she calls "concerted cultivation" (Lareau, 2002, p. 748) whereas working-class and poor parents display a different pattern she names "accomplishment of natural growth" (Lareau, 2002, p. 748). Among others, these patterns differ by parents' language use. Middle-class parents talk more to their children than workingclass and poor parents do. They aim to improve children's vocabulary, elicit utterances and opinions, support their reasoning, and are less directive (Lareau, 2002, pp. 763764). Working-class and poor parents do not focus as much on language as middle-class parents and are more directive. Furthermore, parents following the childrearing pattern of concerted cultivation encourage their children to read during their leisure time (Lareau, 2002, p. 763). In sum, the typical childrearing pattern of middle-class parents (concerted cultivation) aims specifically at developing the children's linguistic skills whereas the childrearing pattern of working-class and poor parents (accomplishment of natural growth) does not explicitly focus on the development of linguistic skills.

Further studies from educational research and psychology also find social class differences in language-related familial socialization. The results of Hart and Risley (1995) are based on data from 42 families across two and a half years by monthly hour-long observations. They show that children's experiences in the family before they are three years old affecting language development are highly correlated with the parental SES (Hart \& Risley, 1995, pp. 148-157) ${ }^{20}$. More specifically, the higher the parents' SES, the higher is the language diversity $(r=.68)$ in terms of the number of different words parents use

\footnotetext{
${ }^{20}$ Measured by the U.S. 1980 census occupational classification (Hart \& Risley, 1995, p. 73).
} 
per hour. Furthermore, the higher the parents' SES, the higher the parental responsiveness, that is parents' responses to children's utterances not evoked by parents $(r=.50)$. A comparison of the children's and their parents' language reveals that they are very similar (Hart \& Risley, 1995, p. 176). $86 \%$ to $98 \%$ of the children's vocabulary use corresponded with parents' vocabulary use. Moreover, the vocabulary growth and vocabulary use of children differs by SES ( $r=.65$ and $r=.63)$.

Hoff (2006) reviews empirical studies on the influence of social context on language development. She concludes that the acquisition of linguistic skills is shaped by the access to communicative opportunities and an analyzable language model provided by children's social contexts. Usually the most influential social context of children regarding their language development are their parents (Hoff, 2006, p. 72). The language-related experiences they give to their children and children's language development vary according to SES. These findings are robust and substantial irrespective of how SES is measured (Hoff, 2006, p. 60). More specifically, SES differences in language-related parenting practices are characterized as follows: higher SES mothers talk more to their children than lower SES mothers, use richer vocabulary, use child-directed speech to elicit conversation with their children more frequently, and give more responses that are related to their children's utterances. Moreover, Hoff et al. (2002, pp. 238-239) report that higher SES mothers' speech is characterized by a greater syntactic complexity. Additionally, higher SES mothers give explicit information more often and talk more frequently about causality than lower SES mothers. Hence, higher SES mothers provide a more beneficial analyzable language model in terms of quantity and quality of speech and more opportunities to communicate for their children than lower SES mothers do.

Moreover, parental reading to children seems to be of special importance with regard to language development. Linberg et al. (2019) decompose the effect of SES on children's linguistic skills in Germany and the US. Besides other factors, daily reading to the child accounts for the linguistic skill gap of about six year old kindergartners in the US and about seven year old first graders in Germany. Higher SES parents read more often to their child. In turn, this promotes children's linguistic skills. In a meta-analysis, Bus et 
al. (1995) find that shared book reading is beneficial for linguistic skills of preschoolers. Demir-Lira et al. (2019) find positive effects of early shared book reading on children's receptive vocabulary, and reading comprehension in elementary school. These effects remain after controlling for other talk to children, SES, and children's early linguistic skills. Moreover, the authors reveal that parental language during shared book reading situations is characterized by a higher vocabulary diversity and syntactic complexity. This contributes to the positive effect of parents' reading to children (see also Bus et al., 1995, p. 2). Most importantly, the effect of shared reading does not depend on parents' SES. Thus, shared reading is equally beneficial for higher and lower SES children (Bus et al., 1995; Demir-Lira et al., 2019).

Crook (1997), de Graaf et al. (2000), and Sullivan (2001) claim that reading furthers skills needed in the educational system, especially linguistic skills. The empirical findings of Sullivan (2001) can be interpreted in that way. ${ }^{21}$ She finds that the positive effect of reading activities on educational achievement (GCSE ${ }^{22}$ results) is completely mediated by linguistic skills and cultural knowledge. Hence, reading activities exert a positive influence on educational achievement only because reading improves linguistic skills and cultural knowledge.

\section{High cultural knowledge}

Becker (2010) studies the intergenerational transmission of cultural knowledge in native and Turkish families in Germany. Drawing on a random sample of 610 German and 627 Turkish families with three to four year old children, results indicate that children with upper-class parents and native German parents have a significantly higher endowment with cultural knowledge than children with lower class parents and Turkish parents have. Analyzing the survey results of 502 adults in Germany, Goßmann and Mätzke (2019) find the same pattern. High cultural knowledge differs significantly by social class and migration background.

\footnotetext{
${ }^{21}$ The studies of Crook (1997) and De Graaf et al. (2000) do not test this assumption empirically.

22 General Certificate of Secondary Education
} 
Again, the question arises why these social differences emerge. Lareau (2002, p. 761) reveals that the parenting pattern of middle-class parents, concerted cultivations, entails the organization of various child leisure activities, including music lessons, choir, arts and crafts classes. These activities are suitable to acquire cultural knowledge in terms of "knowledge of those cultural products from literature, classical music, theatre, and visual arts that are commonly conceived as valuable in a society" (Goßmann \& Mätzke, 2019, p. 4). Although these activities take place outside the families' home, they are organized by the parents that intend to promote their children's development. Participation in such activities is therefore determined by social origin. In contrast, leisure time activities according to accomplishment of natural growth are less organized. They comprise more free, informal play, and do not focus on high culture.

Becker (2010) reveals that the effect of social class on cultural knowledge is mediated completely by family activities, whereas the effect of migration background is partially mediated by family activities. These activities comprise storytelling, reading to their children, visiting zoo or circus, library, museum or theater. Considering Turkish families, Becker (2010) also discloses a positive interaction effect of family activities and the use of German as communication language. In sum, these findings suggest that parents transmit cultural knowledge to their children by family activities with respective cultural content. Surveying 16 year old students in England, Sullivan (2001, p. 904) finds a significant effect of parents' and students' cultural activities on cultural knowledge. However, the effect of students' cultural activities is driven by reading and watching sophisticated television program, but not by high cultural activities.

In sum, empirical evidence shows that linguistic skills are consistently related to social origin across the life course from early childhood to adulthood. This means that social differences are not balanced out by other factors throughout life. There is evidence that social differences are caused by differences in the stimulating potential of parental lan- 
guage use, of the activities parents undertake with their children, and of parenting practices. ${ }^{23}$ The different stimulation potential of the family context exerts its influence from the earliest age. Moreover, there are indications that social origin is associated with cultural knowledge. Higher social class parents more often conduct shared activities that further the acquisition of cultural knowledge than lower social class parents. They also organize such activities outside the home for their children. In addition, higher-class children have higher linguistic skills and cultural knowledge as they read more often than children from lower social classes do.

\subsubsection{The impact of cultural capital on academic achievement}

Cognitive and linguistic skills and knowledge affect academic achievement (for example Bos, Tarelli, Bremerich-Vos, \& Schwippert, 2012; Farkas et al., 1990; Helbig \& Morar, 2017). Teachers assess students according to students' skills and knowledge that teachers convey in school lessons and that students should have learned so far according to academic standards. Academic achievement is the result of assessment according to a fixed evaluation scheme or the teacher's assessment of performance. If one assumes that, the school system should evaluate all students equally regardless of personal characteristics such as social origin, this results in a meritocratic school system.

Bourdieu and Passeron (1977, p. 30) argue that this meritocratic principle causes educational inequalities if students from different social origins are taught in the same manner as educational success requires certain linguistic skills and foreknowledge that are learned exclusively in the upper-class families (compare chapter 2.3.1). This cultural capital is a necessary precondition for getting positive assessments by teachers. Thus, assessments are not the result of individual performance but of social origin.

\footnotetext{
${ }^{23} \mathrm{Hoff}$ et al. (2002) argue that social differences in parenting practices can be explained by parental beliefs and goals that differ by SES. Higher SES parents assume faster development of different skills from their children than do lower SES parents. Moreover, higher SES parents have higher self-efficacy beliefs with regard to their influence on their children's development than lower SES parents. Finally, parents' self-efficacy beliefs are positively related to more ambitious parenting goals and advantageous practices.
} 
In this context, Bourdieu and Passeron (1971, pp. 109-111) emphasize the importance of language. They claim that teachers speak a specific language and expect students to understand and use this language, too. It is a sublime, educated language characterized by a large vocabulary containing a variety of Latin words, a complex sentence structure based on the Latin language, formalism, intellectualism, euphemism, and abstraction. According to Bourdieu and Passeron $(1971,93,114)$, there are basically two mechanisms how the mastery of this educated language affects teachers' achievement evaluations: (1) the passive comprehension of the educated language, and (2) the active, that is written or spoken, production of the educated language.

Regarding the first mechanism, they claim that the pedagogic messages in school are encoded. That is, pedagogic messages are formulated in the educated language. To decode the pedagogic messages, to understand and acquire them, students need to know the respective linguistic code (Bourdieu, 1977a, 493-494). In other words, students need to have sufficient linguistic skills to understand school lessons (see also A. Sullivan, 2001, p. 894). The later mechanism refers to social distinction by language use whether written or spoken (Bourdieu \& Passeron, 1971, pp. 110-115). According to this argument, a person's language use reflects the social origin. Vocabulary, grammatical correctness, syntactical complexity, rhetorical skills, accent, intonation, and the phraseology are signals demonstrating the social class of the family of origin. Regardless of the subject matter, teachers consistently reward the use of the educated language. Therefore, students using the educated language, that are upper-class students, get better grades.

Hoff (2006, p. 69) reviews psychological studies about the relation of the language of the school system and the languages of students from different social backgrounds. She reports that teachers use communicative styles, vocabulary, and grammar similar to (indigenous) middle class families. Depending on social background, students' language matches or mismatches the teachers' language giving them an advantage or penalty with regard to school requirements. Students from lower social background have to learn the language they are confronted with in school whereas higher social background students are already familiar with the language in school. In sum, the findings back the 
view that upper-class students obtain an advantage in school, as the linguistic skills they acquired in their families of origin are much closer to the requirements in school than the linguistic skills of lower-class students. However, results reported by Hoff (2006) do not suggest that only students from the upper social classes can succeed in the educational system. Moreover, she does not refer to social distinction by language, but to socially different evaluation of students according to the ability to meet school requirements.

Bos et al. (2012, pp. 223-224) analyze the 2011 PIRLS study and investigate factors that influence academic achievement in the fourth grade. Academic achievement is measured by the average grade of German, mathematics, and a subject called "Sachunterricht" comprising geography, biology, and history. The authors explain academic achievement by ten independent variables including social and migration background, general cognitive skills, exam anxiety, willingness to achieve, mathematical and reading competence. From the ten factors examined, reading competence has the highest effect on academic achievement. These findings support the assumption that linguistic skills play a central role in the explanation of academic achievement.

Farkas et al. (1990) find that a combined measure of linguistic and mathematical skills affects grades. The results are based on a random sample of 486 seventh and eighth graders in 22 US middle schools in a city school district in school year 1986/87. The language test measures vocabulary, reading comprehension, spelling, capitalization, punctuation, usage and expression. Results of the path model show that linguistic and mathematical skills together have strong total effects on grades in social studies courses. Besides the direct effect, the combined skills measure affect subject specific skills and knowledge ${ }^{24}$ that in turn affect grades. This suggests that linguistic skills have an effect on their own and they affect the acquisition of subject-specific skills and knowledge.

Findings of the PISA study suggest that this also applies for 15 year old students (Autorengruppe Bildungsberichterstattung, 2016, p. 94). Drawing on data of PISA 2000 and

\footnotetext{
${ }^{24}$ Measured by a curriculum-referenced test.
} 
2012 , the authors analyze the relation of the students' reading competence and their German grade for the different school tracks in Germany. In 2000, the correlation coefficients range from $-.14^{25}$ for the lower track (Hauptschule) to -.27 for the upper track (Gymnasium). In 2012, correlations range from -.27 for the intermediate track (Realschule) to -.32 for the lower track (Hauptschule).

DiMaggio (1982) shows that linguistic skills influence US high school grades. Linguistic skills, measured by a vocabulary test, appear to have a positive and strong effect on students' grades in English, history, and mathematics. However, DiMaggio does not use the vocabulary test score as a cultural capital measure, but as a control variable.

Sullivan (2001) examines the influence of another facet of cultural capital on educational success. More specifically, she analyzes the impact of cultural knowledge on the performance of British students in the GCSE. Cultural knowledge is measured by asking students to assign certain persons to the categories politics, music, novels, art or science (A. Sullivan, 2000, appended questionnaire, p. 12-13). Results indicate that cultural knowledge has a significant positive effect on GCSE.

In short, there is evidence that linguistic skills affect academic achievement in primary and secondary schooling. Most studies use vocabulary or reading comprehension tests. Thus, these studies back Bourdieu's idea of the code that is necessary to decipher the pedagogic messages. Indeed, they show that linguistic skills are essential means for the acquisition of substantially relevant skills and knowledge in the school system. Results of Farkas et al. (1990) suggest that active linguistic skills affect academic achievement, too. Literature shows that cultural knowledge has a positive effect on academic achievement and upper-class signals affect teachers' assessments.

${ }^{25}$ Correlation coefficients are negative because in the German educational system lower grades indicate higher achievement. Grades range from 1 (very good respectively "A") to 6 (inadequate respectively "F"). 


\subsection{Contrasting the common and the revised theory}

As pointed out, substantial social inequalities considering linguistic skills exist and linguistic skills affect academic achievement. Hence, disregarding linguistic skills is not reasonable. On the contrary, there is evidence that considering linguistic skills in addition to other relevant factors discussed as cultural capital, habitus or practice is important.

In the following, at first the common theory is presented. A definition of cultural capital is given and hypotheses are formulated. Afterwards the revised theory is described. Based on the definitions in chapter 2.1.3, hypotheses are derived from the state of research presented in chapters 2.2 and 2.3. In addition, the main differences between the revised and the current theory are briefly described. The common and the revised theory outlined in the following are the basis for the empirical comparison in chapter 3.

\subsubsection{The common cultural capital theory}

In order to compare the revised theory with the common theory empirically, hypotheses of the common theory must be clarified. Figure 1 illustrates the theoretical model. The explanatory factors above the dashed line refer to the parents, factors below to the students. Social origin (green) is at the top of the graph, and academic achievement as the central dependent variable (red) is at the bottom. In between are the mediating variables, which are derived from the theory. Straight one-headed arrows represent linear and positive effects. Curved two-headed arrows signify positive correlations. The labels of the paths specify which hypotheses they refer. The suffix ".C" indicates that a hypothesis belongs to the common theory. Additionally, the labels contain a short description of the respective relation by giving a keyword.

The common cultural capital theory is based on DiMaggio's study from 1982 (Goldthorpe, 2007, p. 90; Lareau \& Weininger, 2003, p. 569). In this study, cultural capital is defined as distinctive cultural traits, tastes, and styles of elite status groups (DiMaggio, 1982, p. 189). In accordance with Weber (1978), he claims that the content of status culture is arbitrary. Referring to Bourdieu (1977a; Bourdieu \& Passeron, 1977), DiMaggio specified the prestigious status culture as high culture in terms of art, classical music, and literature (DiMaggio, 1982, p. 191). Hence, cultural capital can be defined as 
positive attitudes towards, participation in, and knowledge about high culture (compare chapter 2.1.1). DiMaggio (1982, p. 190) argues that students possessing this capital can communicate more easily with their teachers, get more attention and support, and are seen as more able than students without this capital.

H1.C: The higher students' high cultural knowledge, the better they are assessed by the teachers.

H2.C: The more positive students' attitudes towards high culture, the more positive they are assessed by teachers.

H3.C: The more students participate in high culture, the more positive they are assessed by teachers.

Moreover, parental cultural capital serves as means of distinction in the school context, too (Aschaffenburg \& Maas, 1997; de Graaf et al., 2000; Rössel \& Beckert-Zieglschmid, 2002).

H4.C: The higher the high cultural knowledge of the parents, the better students are assessed by the teachers.

H5.C: The more positive parents' attitudes towards high culture, the more positive their children are assessed by teachers.

H6.C: The more parents participate in high culture, the more positive their children are assessed by teachers.

Following this perspective, cultural capital by definition must be strictly distinguished from skills (DiMaggio, 1982, 189, 199). It affects academic achievement independent of and in addition to skills (Goldthorpe, 2007, pp. 90-91; Lareau \& Weininger, 2003, pp. 568-569). 


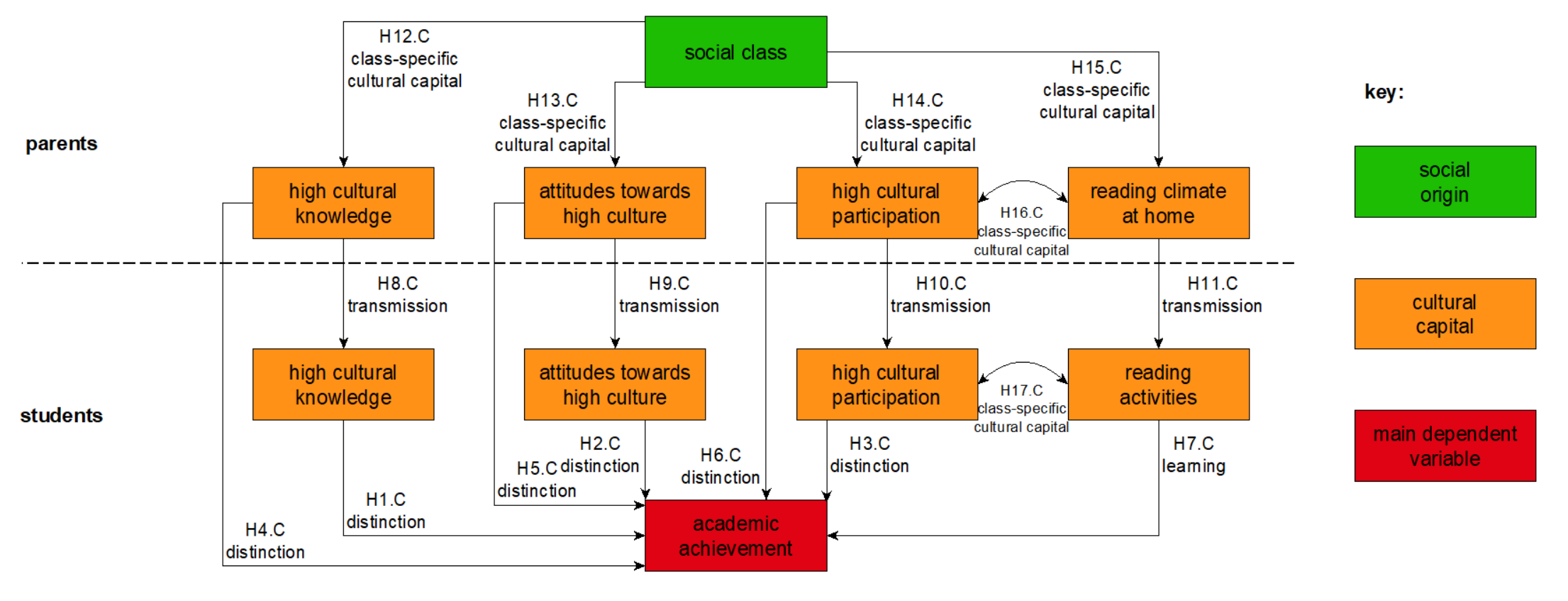

Figure 1: Path model of the common cultural capital theory 
Additionally to high culture, Crook (1997) identifies reading activities as important dimension of cultural capital. Crook (1997) and De Graaf et al. (2000) argue that reading foster skills and knowledge that are needed in the educational system. However, these skills and knowledge are not conceptualized as cultural capital, but human capital (de Graaf et al., 2000, p. 96).

H7.C: The more students read, the better they are assessed by teachers.

Generally, it is assumed that cultural capital is transmitted from parents to their children (Bourdieu, 1977a; de Graaf et al., 2000; Goldthorpe, 2007; Lareau \& Weininger, 2003; Rössel \& Beckert-Zieglschmid, 2002).

H8.C: The higher the cultural knowledge of the parents, the higher the student's cultural knowledge.

H9.C: The more positive parents' attitudes towards high culture, the more positive are the children's attitudes towards high culture.

H10.C: The more parents participate in high culture, the more do their children.

Cultural capital in terms of reading activities is transmitted by the reading climate at home (de Graaf et al., 2000).

H11.C: The more positive the reading climate at home, the more children read.

Moreover, it is claimed that cultural capital differs by social class (Bourdieu, 1977a; de Graaf et al., 2000; Lareau \& Weininger, 2003; Rössel \& Beckert-Zieglschmid, 2002).

H12.C: The higher parents' social class position, the higher the parents' high cultural knowledge. 
H13.C: The higher parents' social class position, the more positive are their attitudes towards high culture.

H14.C: The higher parents' social class position, the more they participate in high culture.

H15.C: The higher parents' social class position, the more positive is the reading climate they provide at home.

De Graaf et al. (2000, p. 98) state that reading behavior and participation in high culture are strongly correlated:

H16.C: The more positive the reading climate at home, the more often parents participate in high cultural activities.

H17.C: The more students read, the more often they participate in high cultural activities.

In sum, the common cultural capital theory postulates two mechanisms that mediate the social class effect on academic achievement: social distinction by high cultural factors and learning by reading activities.

\subsubsection{A revised theory of cultural capital and habitus: hypotheses}

Considering the definitions of cultural capital, habitus, and practice derived in chapter 2.1.3 and the state of the research with regard to those concepts (chapters 2.2.1, 2.2.2, 2.3.1, and 2.3.2), a coherent set of hypotheses can be obtained. These hypotheses are presented in the following. However, the theory specified does not include all the hypotheses that are presented in chapters 2.2 and 2.3. There is a large variety of hypotheses from many different studies with diverging concepts. Hence, incorporating all of these hypotheses into one theory would not result in a coherent and informative theory but an overcomplicated and in parts incoherent accumulation of hypotheses. Therefore, two pathways are focused on that might explain social inequalities in academic achieve- 
ment: high culture-related factors and language-related factors. Thus, this thesis contributes to the widespread and controversial discussion about the role of cultural capital and habitus by picking up commonly used factors and complement them to clarify the underlying mechanisms. The aim of this procedure is to improve the understanding of how inequalities occur and how they could be reduced. Figure 2 shows a path diagram representing the hypotheses of the revised theory. The illustration follows the same pattern as Figure 1 in subchapter 2.4.1. The suffix ,.. $R^{\prime \prime}$ in the labels of the paths signifies that the respective hypothesis is part of the revised theory. Habitus and practice are presented in similar colors (darker and lighter blue) as they are closely related.

\section{High culture}

The students' high cultural knowledge has a positive effect on academic achievement (A. Sullivan, 2001, pp. 907-908). Assuming that high cultural knowledge is typical only for upper social classes, this knowledge becomes a means of social distinction. Teachers perceive students that exhibit such knowledge as members of the educated and cultivated (higher) social classes and, therefore, tend to assess them more positive than students that do not have high cultural knowledge. This effect exists irrespectively of skills and knowledge substantially relevant in lessons.

H1.R: The higher students' high cultural knowledge, the better they are assessed by the teachers.

The parents' high cultural knowledge has a positive effect on achievement evaluation, too. Parents that show high cultural knowledge are also viewed as being educated and cultivated. Hence, their children stem from educated families with a positive educational prospect.

H2.R: The higher the high cultural knowledge of the parents, the better students are assessed by the teachers. 


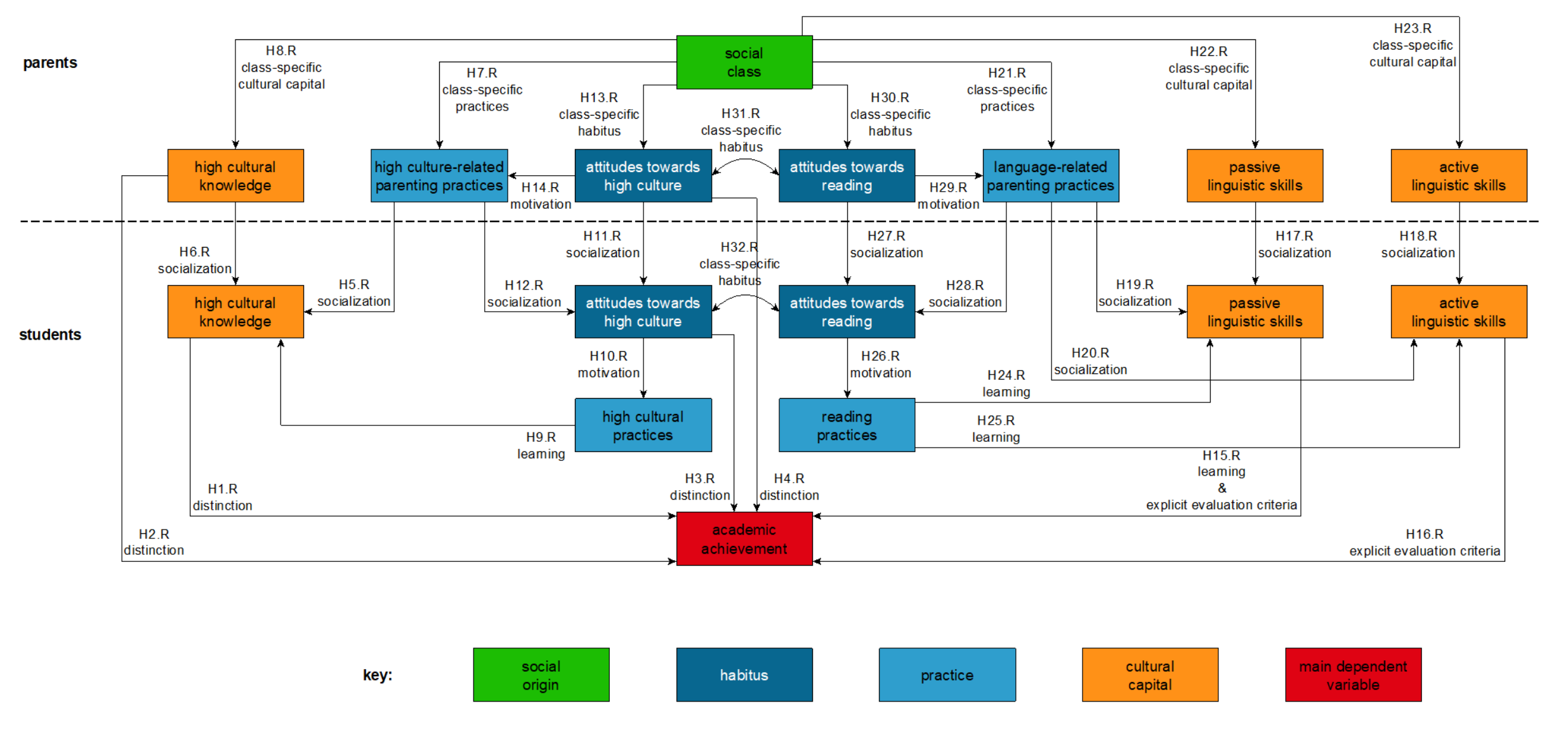

Figure 2: Path diagram of a revised theory of cultural capital and habitus 
This kind of social distinction also operates through attitudes towards high culture. Teachers notice students' or parents' attitudes while interacting with them.

H3.R: The more positive students' attitudes towards high culture, the more positive teachers assess the students.

H4.R: The more positive parents' attitudes towards high culture, the more positive teachers assess the students.

Students gain cultural knowledge by learning processes within the family. Parents pass on their cultural knowledge in everyday interaction with their children and shared high cultural practices (Becker, 2010, pp. 25-26; Lareau, 2002).

H5.R: The more frequently high culture-related parenting practices take place, the higher the student's cultural knowledge.

H6.R: The higher the cultural knowledge of the parents, the higher the student's cultural knowledge.

High culture-related parenting practices (Becker, 2010, p. 24; Lareau, 2002) and parents' high cultural knowledge depend on social class (Goßmann \& Mätzke, 2019).

H7.R: The higher the social class of parents, the more often they do high culture-related parenting practices.

H8.R: The higher the social class of parents, the higher the parents' high cultural knowledge.

High cultural practices like visiting the museum, art exhibitions, theater, opera or classical concerts promote high cultural knowledge. Even though Sullivan (2001, p. 904) does not find such an effect it is reasonable to assume such a relation as high cultural practices provide learning opportunities. 
H9.R: The more frequently students pursue high cultural practices, the higher their high cultural knowledge.

Students carry out high cultural practices if they are sufficiently motivated intrinsically to do so. Attitudes towards high culture are such motivating factors.

H10.R: The more positive students' attitudes toward high culture, the more often the students conduct high cultural practices.

The acquisition of attitudes towards high culture mainly takes place in the home context by familial socialization (Rössel \& Beckert-Zieglschmid, 2002; A. Sullivan, 2001). Parents transmit their attitudes to their children intentionally or unintentionally by activities, discussions, comments etc.

H11.R: The more positive the parents' attitudes towards high culture, the more positive are the students' attitudes towards high culture.

H12.R: The more favorable parents' high culture-related parenting practices, the more positive are the children's attitudes towards high culture.

If high culture is a distinctive characteristic for higher social classes, then members of the higher social classes have more positive attitudes towards high culture than lower social classes.

H13.R: Parents from upper social classes have more positive attitudes towards high culture than parents from lower social classes have.

Moreover, high culture-related parenting practices depend on parents' attitudes towards high culture. Parents who appreciate high culture are more likely to conduct high cultural practices with their children to promote knowledge about and affinity towards high culture.

H14.R: The more positive parents' attitudes towards high culture, the more frequently they engage in high culture-related parenting practices. 
Language

Besides high culture-related factors, language-related factors affect achievement evaluation. Students' linguistic skills have a positive impact on achievement evaluation. At least three possible mechanisms explain this effect. Learning is primarily languagebased. Therefore, (1) linguistic skills are necessary to understand school lessons and to acquire substantially relevant skills and knowledge. This reasoning is in accordance with the concept of the code (Bourdieu, 1977a, pp. 493-494). (2) Language use serves as distinctive signal of the students' social origin (Bourdieu \& Passeron, 1971, pp. 109-115). (3) Students' active linguistic skills are subject of the achievement evaluation (compare Kingston, 2001, p. 97). Bourdieu stresses the first and the second mechanism. Undoubtedly, students need to comprehend the language used in school to learn successfully and get positive assessments. Additionally, Bourdieu states that language use in terms of vocabulary, grammatical correctness, syntactical complexity, rhetorical skills, accent, intonation, and the phraseology (see 2.2.1 and chapter 2.3.2) are distinctive characteristics of the upper social classes. However, it can be argued that some of these characteristics of language use are quite reasonable evaluation criteria as they reflect "the ability to communicate effectively" (Kingston, 2001, p. 97) and to communicate according to general linguistic rules. This especially applies to vocabulary, grammatical correctness, syntactical complexity, rhetorical skills, and choice of words. For example, if teachers evaluate correct grammatical speech or writing positively, this is an obvious criterion and not based on prejudice. However, the acquisition of grammatical rules depends on social origin (Hoff, 2006). It can be assumed that the choice of words also exerts a distinctive effect on academic achievement as well as accent and intonation. However, language comprehension and active linguistic skills are much more important regarding the explanation of social achievement gap than teachers' discrimination based on accent, intonation, or the way students talk.

H15.R: The higher students' passive linguistic skills, the better they are assessed by teachers. 
H16.R: The higher students' active linguistic skills are, the better they are assessed by teachers.

Bourdieu (1977a, pp. 493-494) argues that social inequalities result in differences in the academic achievement as cultural capital, and hence, linguistic skills are almost exclusively acquired in the family of origin. Indeed, there is strong evidence that the family of origin has a large and persistent influence on linguistic skills (see chapter 2.3.1). The language spoken at home by parents serves as an analyzable language model (Hoff, 2006) for children. However, even more important is skill-stimulating interaction of parents with their children from an early age. This gives children access to communicative opportunities (Hoff, 2006) that promote their skills. It is well established that languagerelated parenting practices differ by social class respectively SES. Compared to lowerclass parenting practices, upper-class parenting practices can be described as follows: upper-class parents talk more to their children (Hoff, 2006; Lareau, 2002), use wider vocabulary (Hart \& Risley, 1995; Hoff, 2006; Lareau, 2002) and more complex sentence structure (Hoff et al., 2002), motivate their children more to talk (Hoff, 2006; Lareau, 2002), reply more often to children's utterances (Hart \& Risley, 1995; Hoff, 2006), and read more to their children (Linberg et al., 2019).

H17.R: The higher the passive linguistic skills of the parents, the higher are the student's passive linguistic skills.

H18.R: The higher the active linguistic skills of the parents, the higher are the student's active linguistic skills.

H19.R: The more stimulating parents' language-related parenting practices, the higher are the student's passive linguistic skills.

H20.R: The more stimulating parents' language-related parenting practices, the higher are the student's active linguistic skills. 
Parents' language-related parenting practices (Hart \& Risley, 1995; Hoff et al., 2002; Hoff, 2006; Lareau, 2002; Linberg et al., 2019) and linguistic skills (Maehler et al., 2013, $107,112-113)$ depend on their social class:

H21.R: The higher the social class of the parents, the more favorable are the language-related parenting practices.

H22.R: The higher the social class of the parents, the higher are their passive linguistic skills.

H23.R: The higher the social class of the parents, the higher are their active linguistic skills.

Moreover, reading practices have a positive effect on linguistic skills (McElvany et al., 2008, p. 214; A. Sullivan, 2001, p. 903).

H24.R: The more students read, the higher their passive linguistic skills.

H25.R: The more students read, the higher their active linguistic skills.

Students read more if they have positive attitudes towards reading (L. Baker \& Wigfield, 1999, p. 470; McElvany et al., 2008, p. 214).

H26.R: The more positive students' attitudes towards reading, the more they read.

Students acquire attitudes towards reading in the process of familial socialization. In particular, parents' attitudes (de Graaf et al., 2000) and parents' early reading to children (Demir-Lira et al., 2019) are crucial for the formation of the student's attitudes towards reading. Moreover, it can be assumed that parents with positive attitudes towards reading read more often to their children than parents with less positive attitudes. They might want to pass on to their children the joy of reading that they themselves feel. They might also want to motivate their children to read as they consider reading important for language acquisition and learning. 
H27.R: The more positive parents' attitudes towards reading, the more positive are the student's attitudes towards reading.

H28.R: The more parents read to their children (language-related parenting practices), the more positive are the children's attitudes towards reading.

H29.R: The more positive parents' attitudes towards reading, the more they read to their children.

Parents from higher social classes value reading more than parents from lower social classes (de Graaf et al., 2000).

H30.R: The higher the social class of the parents, the more positive are their attitudes towards reading.

According to the theory of reproduction, there are coherent class-specific forms of habitus (compare chapter 2.2.1). Hence, attitudes towards high culture and reading not only depend on social class, but they are positively correlated, too.

H31.R: The more positive parents' attitudes towards high culture, the more positive are their attitudes towards reading.

H32.R: The more positive students' attitudes towards high culture, the more positive are their attitudes towards reading.

In sum, seven mechanisms of cultural capital and habitus affect academic achievement: social distinction by parents' and students' high cultural knowledge, social distinction by parents' and students' attitudes towards high culture, the learning capacity by passive linguistic skills, and the ability to fulfill explicit evaluation criteria by passive and active linguistic skills. It is assumed that the effects of linguistic skills on academic achievement are stronger than the effects of attitudes towards high culture and high cultural knowledge. Social distinction biases teachers' perception of students' skills and knowledge. Teachers perceive students with an affinity to and knowledge about high 
culture as more educated and able. However, students with high linguistic skills are actually more able in terms of skills that are needed in school.

\subsubsection{Differences between the common and the revised theory}

A comparison of the common and the revised theory reveals that they have some aspects in common. The hypotheses of the common theory that contain high cultural knowledge and attitudes towards high culture can be found in the revised theory, too. Both theories hold that:

(1) Parents from the upper social classes have more high cultural knowledge than parents from the lower social classes have (H12.C, Figure 1 and H8.R, Figure 2 ).

(2) Parents from the upper social classes have more positive attitudes towards high culture than parents from the lower social classes have (H13.C, Figure 1 and H13.R, Figure 2).

(3) Parents pass this high cultural knowledge on to their children (H8.C, Figure 1 and H6.R, Figure 2 ).

(4) Parents pass attitudes towards high culture on to their children (H9.C, Figure 1 and H11.R, Figure 2 ).

(5) Students' and parents' high cultural knowledge have a positive effect on academic achievement (H1.C and H4.C, Figure 1 respectively H1.R and H2.R, Figure 2).

(6) Students' and parents' attitudes towards high culture have a positive effect on academic achievement (H2.C and H5.C, Figure 1 respectively H3.R and H4.R, Figure 2 ).

Apart from these similarities, the theories differ considerably. The definitions of cultural capital diverge. Some concepts that are defined as cultural capital in the common theory (positive attitudes towards and participation in high culture) are defined as habitus or practice in the revised theory. 
The main differences between the common and the revised theory are:

(1) The common theory is more parsimonious than the revised theory. The interpretation of the common theory presented in this thesis contains 17 hypotheses whereas the revised theory contains 32 .

(2) In contrast to the common theory, the revised theory considers linguistic skills to explain the relation of social origin and academic achievement.

(3) The revised theory describes the acquisition of factors considered as relevant for the explanation of social differences in academic achievement in more detail than the common theory. More specifically, parenting practices that promote attitudes towards high culture, attitudes towards reading, high cultural knowledge, and linguistic skills are taken into account.

(4) The revised theory contains attitudes that differ by social class and lead to social differences in achievement-relevant behavior. The common theory does not explain behavior that differs by social class. 


\section{Explaining social inequalities in academic achievement: An empirical comparison}

The aim of the following chapter is to compare the explanatory potentials of the two competing theories of cultural capital regarding the social differences in academic achievement (as described in chapter 2) empirically. This comparison is especially important to evaluate the usefulness (see chapter 1 ) of the competing theories.

The chapter is structured as follows. First, the analytical sample is described. Then, the variables chosen for the analytic models are presented and the handling of missing data is explained. Next, the analytical strategy is depicted. Thereafter, the model specifications, statistical power, model fit evaluations, and the empirical results of the models are presented. In order to meet the analytical requirements, multi-group path analyses are performed. Results are presented in chapter 3.5.7.

\subsection{Sample}

For the analyses, data from Starting Cohort 3 (SC3) wave 1 from the National Educational Panel Study (NEPS) are used. The target population consists of students in schools of lower secondary education in Germany in the school year 2010/11. SC3 wave 1 is sampled in a two-stage procedure, this means that schools are selected and then classes. Moreover, the sample is stratified and has an overlap with Starting Cohort 4 (SC4). Schools that educate students in the fifth and ninth grade as well as special-needs schools are sampled in SC3 and SC4. Additionally, SC3 contains schools that educate students in the fifth grade, but not in the ninth grade. This part of the SC3 sample does not overlap with SC4 (Steinhauer \& Zinn, 2016, pp. 3-4).

The data set of SC3 wave 1 contains information about 6,112 students in the fifth grade. For the analyses, some observations are discarded. Students of special-needs schools are excluded from the sample (587 cases) as (1) they are a very particular population for whom it cannot be assumed that the mechanisms underlying grading are the same as for students of schools of general education. (2) The data lack some of the crucial variables for the respective subsample, that is, competence measures. To ensure that data 
contain sufficient information for the imputation procedure only those cases are included that at least contain information from the students' interview and competence tests in wave 1. It can be assumed that imputation based on sample information and information from the parent interview does not lead to trustworthy results. For this reason, 925 cases are excluded. Further observations have to be excluded to meet the requirements of multiple imputation to handle missing data due to item non-response according to Drechsler (2015). The imputation models contain the school cluster identifier to account for school effects. If the number of observations within a school cluster is below 10 imputed data might be biased. Hence, students nested in school with less than 10 observations are excluded. Therefore, 450 observations are excluded. Furthermore, students are excluded that did not receive a grading in German or mathematics in the fifth grade according to the students' paper and pencil interviews (PAPI) as this is the most important dependent variable of the analyses (182 respectively 153 cases). After the exclusion according to the above-mentioned conditions, 4,924 of 6,112 cases (80.6\%) in wave 1 remain.

Observations with missing values in the German grade and the mathematics grade in the fifth grade, that is, the basis of the dependent variable of major interest, are excluded neither (1) from the imputation model nor (2) from the analysis model. These observations are included in the imputation model (1) as they are predictive considering the relationships between the independent (exogenous) variables. They are included in the analysis models (2) as data contain auxiliary variables predicting grades in the fifth grade very well, for example grades in the fourth, seventh, and ninth grade.

\subsection{Variables}

In the following, the variables used in the analytical models are described. The descriptive results reported are based on the observed NEPS SC3 variables before imputation.

\section{Average grade in German and mathematics}

To reflect the influences of the explanatory factors of academic achievement postulated by the theories an indicator based on the subjective assessments of teachers is needed. 
In this way, the influences of social distinction, reading, and linguistic skills on academic achievement can be analyzed. Grades represent such assessments of teachers. The average grade in German and mathematics was chosen, as German and mathematics are major subjects in all types of school that are central to a successful school career. First, grades have a sorting function (Henninges, Traini, \& Kleinert, 2019). Tracking after primary schooling is based on grades. Moreover, students with poor grades that do not reach the required standard have to repeat the grade or even have to go to a lower track if they attend the intermediate (Realschule) or higher track (Gymnasium). Second, the school subjects German and mathematics represent a broad spectrum of academic demands on the students. At the same time, linguistic skills play a role in the acquisition of mathematical skills (Heinze, Herwartz-Emden, \& Reiss, 2007). For these reasons, the average grade of the students in German and mathematics from the annual report of the fifth grade was used as an indicator of academic achievement.

The data contain two measures of this information. Grades are surveyed by the students' PAPI and by the computer assisted telephone interview (CATI) of the parents. The information from the students' PAPI is used as it is assumed that (1) students probably remember grades better than their parents do and (2) the social desirability effect is presumably higher in a personal interview situation as in the parent's CATI than in a more anonymous paper and pencil interview situation.

In the secondary schooling in Germany, grades range from one to six. Grade one implies that the performance notably meets requirements. Grade six means that the performance does not meet requirements and reveals incomplete basic skills and knowledge. For reasons of international comprehensibility, the coding is reversed. The German educational system is determined by the education policy of the 16 Federal States. It is complex and many different types of school exist. This is especially true for the fifth grade. Hence, the variable average grade in German and mathematics is standardized within the respective federal state and type of school to account for differences in scales across federal states and types of school using the following formula. 


$$
Z_{i_{k}}^{j}=\frac{Y_{i}-\bar{Y}_{k}^{j}}{S D\left(Y_{k}^{j}\right)}
$$

$Z_{i}^{j}$ is the standardized average grade of the student $\mathrm{i}$ in the federal state $\mathrm{j}$ and the type of school k. $Y_{i}$ is the observed average grade of the student i. $\bar{Y}_{k}^{j}$ is the mean of the average grade in the federal state $\mathrm{j}$ and the type of school k. $S D\left(Y_{k}^{j}\right)$ is the standard deviation of the average grade in the federal state $\mathrm{j}$ and the type of school k. The resulting average grade has a mean of 0.00 and a standard deviation of 0.98 (Table 4).

\section{Parental education}

There are several indicators for social origin based on income, occupation, and formal education. Since occupational and education-related indicators are strongly interrelated (compare Appendix, Table 14), only one very differentiated measurement is used for the formal education of parents. Moreover, parental education seems to be particularly suitable as it is closely associated with family resources, environment, values, and beliefs (Bradbury \& Corak, 2015, p. 25). These aspects are highly relevant for academic achievement. Data on parental education are available for both parents. It is measured in the parents' CATI. The variable used for the analyses represents the formal education of the parent with the higher educational certificate. The highest formal education of the parents is differentiated as follows: lower secondary (Hauptschulabschluss), intermediate secondary (Mittlere Reife), higher secondary (Abitur), lower tertiary (Fachhochschulabschluss), and higher tertiary education (Hochschulabschluss). Table 4 shows the distribution of parental education. About $12 \%$ have a lower secondary school leaving certificate whereas $22 \%$ have a university degree. For the path analyses, parental education is transformed into five dummy variables.

\section{Number of books in the home}

Testing the common cultural capital model, the reading climate at home is measured by the number of books in the home. It can be interpreted as (1) an opportunity structure for reading and (2) a material manifestation of the parents' attitudes towards reading. 
Parents who enjoy reading are more likely to buy books. Hence, the number of books in the home is a suitable proxy for the reading climate at home.

Considering the revised cultural capital model, books in the home is used as proxy variable for parents' attitudes towards reading and parental linguistic skills as data lack direct measures. Clearly, books in the home is a rough proxy measure for parental linguistic skills. However, books in the home represent the parental reading behavior across their life course, which is conducive to their linguistic skills (compare chapter 2.3.1). From this perspective, the variable seems to be the best measure for parental linguistic skills available. ${ }^{26}$

This information is collected in the survey of students and parents. It can reasonably assumed that the responses of the parents are more accurate than the responses of the students aged between ten and twelve. Therefore, the respective information surveyed by the parents' CATI is used as an indicator. Parents are asked how many books they have about in their homes. Response options are: 1: 0 to 10 books, 2: 11 to 25 books, 3: 26 to 100 books, 4: 101 to 200 books, 5: 201 to 500 books, 6: more than 500 books (Goßmann, 2018, 8, 10). For more favorable statistic properties, the variable books in the home is treated as quasi-metric (mean $=4.15 ; \mathrm{SD}=1.27$; compare Table 4). Bivariate regression analyses $(\mathrm{OLS})^{27}$ of the various dependent variables in the models on books in the home show that neither R-squares nor root mean squared errors are considerably more favorable when books in the home is included as categorical variable.

\section{Students' and parents' high cultural participation}

High cultural participation is typically used as measures of social distinction in the common cultural capital theory (compare de Graaf et al., 2000, p. 96). Thus, this measurement is implemented in the analysis of the common cultural capital model.

\footnotetext{
${ }^{26}$ Formal education of the parents is assumed not to be a better proxy for linguistic competencies than books in the home since books have a stronger relation to language than general formal education.

${ }^{27}$ Ordinary Least Squares
} 
Data lack measures of high cultural knowledge and attitudes towards high culture. Hence, high cultural participation is used as a proxy for attitudes towards high culture in the empirical model of the revised theory. Even though high cultural practices seem to be related to high cultural knowledge as well (see chapter 2.4 .2 ), the empirical correlation of high cultural practices with attitudes towards high culture is assumed higher than with high cultural knowledge.

Students and their parents are asked how often they have done the following things in the past twelve months: (1) visited a museum or an art exhibition, (2) visited an opera, ballet or classic concert, and (3) been to the theater. Response options are: 1: never, 2: once, 3: 2 to 3 times, 4: 4 to 5 times, 5: more than 5 times (Goßmann, 2018, pp. 11-12). The variables indicating participation in high cultural participation are sum scores based on these three items (scale of students: mean $=6.15 ; \mathrm{SD}=2.47$; scale of parents: mean =6.20; $\mathrm{SD}=2.64$; compare Table 4). The scales of students and parents are reliable (scale of students: Cronbach's $\alpha=0.646$; scale of parents: Cronbach's $\alpha=0.692$ ), uni-dimensional, and valid (Goßmann, 2018, pp. 39-51).

\section{Attitudes towards reading}

As argued above, attitudes towards reading are a dimension of habitus that is crucial for the explanation of individuals' reading behavior (compare chapter 2.2.2). In terms of habitus, it is appropriate to operationalize attitudes towards reading as intrinsic aspect of motivation that is directly related to reading. According to the concept of habitus, dispositions that govern practice are more affective rather than being instrumental (compare chapter 2.1.2). The chosen data contain a scale reflecting the respondents reading pleasure (Möller \& Bonerad, 2007). The scale is surveyed in the students' PAPI by a Likert scale with the following stimuli around the question "What do you think about reading?":

I enjoy reading books.

I find reading interesting. 
If I had enough time, I would read even more.

Students rate those items on a four-point scale ranging from 1 (completely disagree) to 4 (completely agree). The scale displays a satisfactory reliability coefficient (Cronbach's $\alpha=0.90)$. To assess uni-dimensionality a confirmatory factor analysis is conducted. Results show that factor loadings on the common factor range between 0.76 and 0.91 . In addition, the items show no considerable factor loadings $(\geq 0.3)$ on further factors. Therefore, these results indicate uni-dimensionality of the scale. The variable attitudes towards reading is generated as a sum score of the three items (mean $=9.20 ; S D=2.75$; compare Table 4).

\section{Time spent on reading}

Reading is part of the common and the revised cultural capital model as it represents an activity respectively practice that furthers the development of skills. This is especially true for linguistic skills (A. Sullivan, 2001). It is assumed that any kind of reading is potentially beneficial for the development of linguistic skills, reading activities are operationalized by the time students spent on reading outside school irrespectively of what they read (Goßmann, 2018, pp. 16-17). Students are asked in the PAPI how much time they usually spend reading outside of school (1) on a normal school day and (2) on a normal non-school day. There are five response options: 1: not at all outside school, 2: up to half an hour, 3: between half an hour and 1 hour, 4: 1 to 2 hours, 5: more than 2 hours. The variable is calculated as a quasi-metric weighted mean based on a typical week with 5 school days and 2 days off (mean $=0.87 ; S D=0.64$; compare Table 4).

\section{Reading comprehension}

According to the revised theory, passive linguistic skills are crucial for academic achievement (compare chapter 2.4.2). Passive linguistic skills can be divided into listening and reading comprehension. Data on reading comprehension are available for grades five, seven, and nine, whereas listening comprehension is surveyed only in grade nine. Therefore, reading comprehension is used. 
The reading competence test in the fifth grade is a paper and pencil test and comprises 32 items that have different cognitive requirements (finding information in text, drawing text-related conclusions, and reflecting and assessing) and text functions (information texts, instruction texts, advertising texts, commenting or argumenting texts, and literary texts; Pohl, Haberkorn, Hardt, \& Wiegand, 2012, p. 5). Pohl et al. (2012) analyze the quality of the scale and conclude that the test shows good psychometric properties regarding item fit, measurement invariance across subgroups, reliability, and uni-dimensionality. However, the test mainly aims at low-performing students. The relative share of correct responses ranges between $26 \%$ and $94 \%$ with an average of $69 \%$ (Pohl et al., 2012, p. 17). Therefore, the test does not adequately measure reading comprehension of high-performing students.

The data provide weighted maximum likelihood estimates (WLE). WLEs are point estimates of the individual competence scores (Pohl \& Carstensen, 2012, p. 16). They account for missing responses and allow for comparison of the competence scores across different waves. The WLE score of reading comprehension has a mean of 0.02 and a standard deviation of 1.25 (compare Table 4).

\section{Orthographic competence}

Orthographic competence is used to measure active linguistic skills (compare chapter 2.4.2). The ability to write correctly in the teaching language is essential for the written language and for the assessment of students by teachers.

The test of orthographic competence is a paper and pencil test and consists of the following five dimensions (Frahm et al., 2011, p. 226). (1) The phonographic and syllabic principle refers to the capability to understand the corresponding syllabic structure of written and spoken words. (2) The morphological principle stands for the understanding of the structure of words in inflected and derived forms. (3) The peripheral area concerns the knowledge of exceptions in spelling and the correct spelling of foreign words. (4) The word formation principle relates to the familiarity with parts of speech and derivational morphemes. (5) The syntactic principle denotes the knowledge of syntactic 
structure for capitalization. The test consists of a cloze test with 30 words and three full sentences with 40 words. The test instructions and words are spoken on tape by a professional speaker and played during the test.

The empirical results (Blatt, Frahm, \& Jarsinski, 2013) show that the test is reliable and has varying item difficulties. Five latent factors represent subskills and correspond with the dimensions described above. However, those factors highly correlate. Therefore, the WLE estimator is used. The WLE estimate of orthographic competence has a mean of 0.04 and a standard deviation of 1.36 (compare Table 4).

\section{General cognitive skills: perceptual speed and reasoning}

In all statistical models analyzing the determinants of reading comprehension, orthographic competence, and grades, the influence of non-verbal general cognitive skills is controlled. Non-verbal general cognitive skills are more strongly determined by genes than by experience, culture, and language (Leibniz Institute for Educational Trajectories, National Educational Panel Study, 2018, p. 11). Both measures of general cognitive skills, perceptual speed and reasoning, are available in the data. They are measured by paper and pencil tests. Perceptual speed is an indicator for the basal speed of information processing whereas reasoning is an indicator for fluid intelligence. The sum score of perceptual speed has a mean of 44.08 and a standard deviation of 13.33 (compare Table 4). The mean of the sum score of reasoning amounts to 6.97 and the standard deviation equals 2.60 (compare Table 4).

\section{Student's sex}

To account for gender-specific differences student's sex is controlled for in the path models. Data on the students' sex are available as sample information and additionally surveyed by the students' PAPI and the parents' CATI. The variable used, is based on the respective information of the parents' survey, as it seems to be the most valid one. However, data from the parents' interview contain a considerable amount of missing values due to unit nonresponse. Hence, missing values of the variable are replaced by sample 
information. The variable is coded as a dummy indicating if students are male $(0=$ false; 1 = true; $M=0.51, S D=0.50$, see Table 4).

\section{Migration background}

In all statistical models, the influence of the migration background is controlled. The variable used is surveyed in the parents' CATI. Students that are born abroad or have at least one parent or parent's parent born abroad are defined to have a migration background. Migration background is dummy-coded ( 0 = false; 1 = true; $M=0.30 ; S D=0.46$ ).

\section{Type of school}

When regressing the standardized average grade in the fifth grade, type of school is controlled to account for differences in means between various types of school. As mentioned above, the German educational system is complex. Overall, up to seven categories of types of school can be identified by sampling information, which can be assumed as valid.

The recoding of the variable in the sense of a grouping of categories is based on the tracks offered by the respective schools. In grade five, there are four types of school tracks: a lower secondary track (Hauptschule and the lower track at schools with several educational programs), an intermediate secondary track (Realschule and the intermediate track at schools with several educational programs), a higher secondary track (Gymnasium) and types of school without tracking. The latter comprise the fifth grade at elementary schools in the federal states of Berlin and Brandenburg, the orientation stage and integrated Gesamtschule. The type of school variable represents tracking for several reasons: Tracking implies different levels of educational requirements and differing social compositions and levels of academic achievement. Hence, secondary schools with tracking below higher secondary school (Gymnasium) are combined in one category (lower and intermediate secondary school, school with several educational programs). Higher secondary school (Gymnasium) is a category of its own. Elementary school, orientation stage and integrated Gesamtschule are conflated into another category 
(schools without tracking). About $40 \%$ of the students in the analytical sample visit a school with a lower and / or intermediate track, $46 \%$ attended a higher track, and about $14 \%$ visit a school without tracking (compare Table 4).

Table 4: Descriptive values of dependent and independent variables

\begin{tabular}{l|lllll}
\hline & Obs & Min & Max & Mean & SD \\
\hline Average grade & 4,239 & -3.64 & 3.03 & 0.00 & 0.98 \\
Parental education: lower sec. & 3,653 & 0 & 1 & 0.12 & 0.32 \\
Parental education: intermed. sec. & 3,653 & 0 & 1 & 0.34 & 0.47 \\
Parental education: higher sec. & 3,653 & 0 & 1 & 0.22 & 0.41 \\
Parental education: lower tertiary & 3,653 & 0 & 1 & 0.10 & 0.30 \\
Parental education: higher tertiary & 3,653 & 0 & 1 & 0.22 & 0.42 \\
Books in the home & 3,480 & 1 & 6 & 4.15 & 1.27 \\
High cultural participation (parent) & 3,482 & 3 & 15 & 6.20 & 2.64 \\
High cultural participation (student) & 4,164 & 3 & 15 & 6.15 & 2.47 \\
Attitudes towards reading & 4,483 & 3 & 12 & 9.20 & 2.75 \\
Time spent on reading & 4,272 & 0 & 2.02 & 0.87 & 0.64 \\
Reading comprehension & 4,909 & -4.59 & 4.07 & 0.02 & 1.25 \\
Orthographic competence & 4,924 & -5.69 & 4.45 & 0.04 & 1.36 \\
Perceptual speed & 4,920 & 0 & 93 & 44.08 & 13.33 \\
Reasoning & 4,904 & 0 & 12 & 6.97 & 2.60 \\
Student's sex: male & 4,924 & 0 & 1 & 0.51 & 0.50 \\
Migration background & 3,667 & 0 & 1 & 0.30 & 0.46 \\
Type of school: lower \& intermed. sec. & 4,924 & 0 & 1 & 0.40 & 0.49 \\
Type of school: higher secondary & 4,924 & 0 & 1 & 0.46 & 0.50 \\
Type of school: without tracking & 4,924 & 0 & 1 & 0.14 & 0.34 \\
\hline Soure: owncal & & & & &
\end{tabular}

Source: own calculations based on NEPS SC3 7.0.0

It has to be considered that the measures used stem partially from different sources and times of measurement. Students have been surveyed and tested between November 2010 and January 2011. During this period, the following information had been collected by PAPI: students' high cultural participation, attitudes towards reading, time spent on reading, reading comprehension, orthographic competence, and perceptual speed. 
Moreover, parents had been interviewed via CATI from January 2011 to July 2011. Information on parental education, books in the home, parents' high cultural participation, students' sex, and migration background had been collected in these interviews. Additionally, sampling information about the type of school and the students' sex are used. The crucial dependent variable is students' grades of the final report card of the fifth grade. Students receive the final report card at the end of the school year. The grades in the final report card represent a weighted mean of the grades students got during the whole school year in the respective school subject. Depending on the federal state, school years end between the end of June and the end of July. Hence, at least half of the single grades that form the basis of the grades in the final report card had been assigned after the students' survey. Besides quite strong theoretical arguments for the claimed causal direction, this makes reversed causality rather unlikely. Data on grades of the fifth grade are collected in the students' survey of wave 2 . The interviews took place between November 2011 and January 2012. In some cases, parents' interviews are from the time when the school year ended. However, most of the characteristics surveyed by parents' interviews are quite stable across time. This applies for parental education, books in the home, students' sex, and migration background. Parents' high cultural participation might be more time variant. However, it measures cultural activities from the 12 months before the interview. Thus, it is possible that some of the measured behavior took place before some of the grades were assigned.

\subsection{Missing data}

Overall, $47 \%$ of the observations in grade five are completely observed regarding the 14 variables of the revised model. In case of the common model, $49 \%$ of the observations are completely observed. Generally, using complete cases might lead to biased results. Complete cases analysis is unbiased only if the Missing Completely at Random (MCAR) assumption is met (Rubin, 1976). This means that the probability of missingness is the same for all observations. To test if the MCAR assumption holds regarding the given data, the Little's test is conducted using the R function LittleMCAR from the BaylorEdPsych package, based on Little (1988). It tests the null hypotheses that the missing 
data are MCAR. The $p$-value is 0.000 indicating that the missing data are not MCAR. Therefore, analyzing complete cases and ignoring missing data due to item-nonresponse leads to biased results.

To avoid this bias, multiple imputation is used, which was initially developed by Rubin (1987). Multiple imputation leads to valid values if missingness depends only on observed data, that is, that the Missing at Random (MAR) assumption holds (Rubin, 1976). If missingness depends on unobserved data, also called Not Missing at Random (Rubin, 1976) the resulting values might not be valid. Unfortunately, testing for NMAR empirically is not possible. Multiple Imputation is preferred to the full information maximum likelihood method (FIML) for the following reasons(Teman, 2012, p. 52):

(1) A variety of auxiliary variables is given in the data set.

(2) Variables with different levels of measurement are included, that are easier to handle with multiple imputation than with FIML.

(3) Exogenous variables are heavily inflicted by missing data requiring auxiliary variables.

Multiple imputation by chained equations (MICE) ${ }^{28}$ is applied using Stata. Using MICE, a missing value is replaced (=imputed) by several (=multiple) predicted values. This takes into account the uncertainties associated with the missingness. Multiple imputation generates $M$ completed data sets. To determine the number of imputations $M$ that should be used White et al. (2011) suggest that M should be at least equal to the proportion of observations containing missing values. According to Graham (2007), the fraction of missing information has to be taken into account which can be interpreted as a measure of uncertainty. Taking into account both sources, 100 completed data sets are

\footnotetext{
${ }^{28}$ More information about MICE in general can be found in Raghunathan et al. (2001) and Van Buuren (2007) with Van Burren as the inventor of the MICE algorithm.
} 
generated. The completed data sets can be analyzed by standard procedures. Afterwards, the estimates are calculated using Rubin's combining rules (Rubin, 1987).

In addition to variables contained in the analytical models (see chapter 3.2), a variety of auxiliary variables is used for imputation: grades reported by the parents, mathematical competence, number of books in the home reported by the students, students' age, and sampling information, that is strata. Moreover, measurements of the variables in later waves (leading values) are also included, as these values are usually good predictors of the missing values.

Von Hippel (2007) argues that cases with missing values in the dependent variables should be included into the imputation model but not into the analytical model (the so called multiple imputation, then deletion (MID) approach). This is because these cases contain information on the relationship between the independent variables, but they do not contain any information on the relationship between the dependent and independent variables. However, Sullivan et al. (2015) show that MID should not be used if auxiliary variables are included in the imputation model that have high predictive power. As the given data contains various auxiliary variables with a high predictive power, MID is not implemented. Instead, cases with missing values in the dependent variables are included in the analytical models.

To address the multi-level structure of NEPS SC3 data, cluster fixed-effects on the school level are applied (Drechsler, 2015). In other words, a set of dummy variables is included that indicates which school a specific student attends. As small cluster sizes may bias the imputed data, observations are discarded if cluster size is smaller than 10 (Drechsler, 2015, pp. 89-90). In wave 1, this is true for 263 students.

Regarding continuous variables, predictive mean matching (PMM) is favored over linear regression as PMM is more robust to misspecification of the imputation model and violations of the distributional assumptions (White et al., 2011). Furthermore, using PMM prevents the imputed values from being beyond the range of the observed data. In contrast, when using imputation by linear regression this problem may occur. For each case 
with a missing value, PMM identifies similar cases in the data that have observed values and imputes one out of these observed values. First, PMM matches cases with missing values and cases with observed values. PMM matches those $\mathrm{k}$ cases with observed values to a case with a missing value that have the most similar values predicted by linear regression. Second, PMM randomly choses one out of the $\mathrm{k}$ cases and assigns its observed value to the case with the missing value. The number of potential donor values from observed data is set to $k=10$, following the recommendation of Morris et al. (2014). Ordinal variables are imputed using ordered logistic regressions; binary variables are imputed by logistic regressions.

Parental education is surveyed by the parents' interview. The share of unit nonresponse in the sample used for the analyses amounts to $29.26 \%$ in the first wave. Since NEPS SC3 is a panel study, some of the non-respondents of the first wave participate in later waves. Moreover, parents' education is a relatively stable characteristic. Hence, data on parents' education from the next observation available is used if it is missing in wave 1.

To determine whether the usage data on parents' education from the next available wave is suitable, the relative share of changes is computed in the variables education of the respondent and education of the respondents' partner across wave 1 to wave 7 . The same categorization of education is used as for the analytic models. Moreover, only those respondents are referred to that are interviewed at least twice $(n=3,346)$ respectively to those partners of whom at least two observations are available $(n=3,190) .81$ changes in the respondents' education are reported across wave 1 to 7 . This means that out of the respondents that are surveyed at least twice $2.4 \%$ have a change in the variable education. Regarding the respondents' partners, 73 changes occur. Hence, $2.2 \%$ of the respondents' partners have a change in this variable. These relative shares approximate the measurement error that is induced by the procedure. However, it can be assumed that this error is smaller than the error that would occur by multiple imputation with chained equations. Therefore, the procedure to replace missing data in the variable parental education by information from later waves is assumed as suitable. 
Overall, the relative share of missing values in the variable parental education is reduced from $29.45 \%$ to $25.81 \%$. This difference seems to be small. However, Table 5 shows the rates of change that indicate considerable differences, with the rate of change computed as follows:

$$
\text { rate of change }=\frac{\text { education }_{w 1 \text { to } w 7}-\text { education }_{w 1}}{\text { education }_{w 1}} * 100
$$

First, the share of missing values is reduced by $12.34 \%$ considering the number of missing values before using information from later waves. Secondly, changes due to the procedure are not equal across the different levels of education. The highest rate of change occurs with regard to parents holding a lower secondary school leaving certificate (8.00\%), followed by parents that had a lower $(6.03 \%)$ respectively higher tertiary degree (5.97\%). Hence, the applied procedure to replace missing values in the variable parental education generates additional information on this crucial variable especially at the lower and the upper end of the distribution.

Table 5: Rate of change of parental education

\begin{tabular}{lccc}
\hline Parental education & Wave 1 & Wave 1 to wave 7 & Change (\%) \\
\hline Missing value & 1,447 & 1,271 & -12.34 \\
Lower secondary & 400 & 432 & 8.00 \\
Intermediate secondary & 1,190 & 1,243 & 4.45 \\
Higher secondary & 768 & 792 & 3.13 \\
Lower tertiary & 348 & 369 & 6.03 \\
Higher tertiary & 771 & 817 & 5.97 \\
\hline Total & 4,924 & 4,924 & \\
\hline
\end{tabular}

Source: own calculations based on NEPS SC3 7.0.0

Note: The table shows absolute numbers.

Table 6 shows the means and standard deviations of the variables in the analytical model for the observed and the imputed data. Overall, there are no big differences between observed and imputed data with regard to means and standard deviations. Orthographic competence, students' sex, and the type of school are completely observed. Hence, the 
distribution does not alter. The mean and the standard deviation of the average grade remain unchanged as it is standardized. Moreover, no changes occur in the share of parents with lower tertiary education, and the means of reading comprehension.

Table 6: Comparison of descriptive statistics of observed and imputed data

\begin{tabular}{|c|c|c|c|c|c|c|}
\hline & & & Observ & & Impute & \\
\hline & Obs & $\begin{array}{l}\text { Missing } \\
\text { rate }\end{array}$ & Mean & SD & Mean & SD \\
\hline Average grade & 4,239 & 13.91 & 0.00 & 0.98 & 0.00 & 0.98 \\
\hline Parental education: lower secondary & 3,653 & 25.81 & 0.12 & 0.32 & 0.14 & 0.35 \\
\hline Parental education: intermediate secondary & 3,653 & 25.81 & 0.34 & 0.47 & 0.36 & 0.48 \\
\hline Parental education: higher secondary & 3,653 & 25.81 & 0.22 & 0.41 & 0.21 & 0.41 \\
\hline Parental education: lower tertiary & 3,653 & 25.81 & 0.10 & 0.30 & 0.10 & 0.29 \\
\hline Parental education: higher tertiary & 3,653 & 25.81 & 0.22 & 0.42 & 0.20 & 0.40 \\
\hline Books in the home & 3,480 & 29.33 & 4.15 & 1.27 & 4.03 & 1.29 \\
\hline High cultural participation (parent) & 3,482 & 29.29 & 6.20 & 2.64 & 6.05 & 2.59 \\
\hline High cultural participation (student) & 4,164 & 15.43 & 6.15 & 2.47 & 6.14 & 2.47 \\
\hline Attitudes towards reading & 4,483 & 8.96 & 9.20 & 2.75 & 9.14 & 2.77 \\
\hline Time spent on reading & 4,272 & 13.24 & 0.87 & 0.64 & 0.85 & 0.64 \\
\hline Reading comprehension & 4,909 & 0.30 & 0.02 & 1.25 & 0.02 & 1.25 \\
\hline Orthographic competence & 4,924 & 0.00 & 0.04 & 1.36 & 0.04 & 1.36 \\
\hline Perceptual speed & 4,920 & 0.08 & 44.08 & 13.33 & 44.07 & 13.33 \\
\hline Reasoning & 4,904 & 0.41 & 6.97 & 2.60 & 6.96 & 2.60 \\
\hline Student's sex: male & 4,924 & 0.00 & 0.51 & 0.50 & 0.51 & 0.50 \\
\hline Migration background & 3,667 & 25.53 & 0.30 & 0.46 & 0.31 & 0.46 \\
\hline Type of school: lower \& intermediate secondary & 4,924 & 0.00 & 0.40 & 0.49 & 0.40 & 0.49 \\
\hline Type of school: higher secondary & 4,924 & 0.00 & 0.46 & 0.50 & 0.46 & 0.50 \\
\hline Type of school: without tracking & 4,924 & 0.00 & 0.14 & 0.34 & 0.14 & 0.34 \\
\hline
\end{tabular}

Source: own calculations based on NEPS SC3 7.0.0

There are slight decreases in the share of parents with higher secondary education and higher tertiary education, the means of books in the home, parents' and students' high cultural participation, attitudes towards reading, time spent on reading, perceptual 
speed, and reasoning. The share of parents with lower and intermediate secondary education, and migration background slightly increase. Furthermore, little changes in the standard deviations of parental education, books in the home, parents' high cultural participation, and attitudes towards reading arise.

\subsection{Analytical strategy}

The common and the revised theory, as outlined in chapters 2.4 .1 and 2.4 .2 , contain a complex structure of causal hypotheses with a variety of dependent and independent variables. According to these theories, several causal paths mediate the effect of social origin on academic achievement. Path analysis is therefore a suitable method for checking these complex theories, but not for confirming causality empirically. It is rather a method to test theoretically founded causal models on the basis of empirical data (Bortz \& Schuster, 2010, p. 435).

Model fit plays an important role in path analysis. If a model has an inadequate model fit, it is considered as falsified. If, on the other hand, model fit is sufficient, the model is said to be provisionally confirmed (Bortz \& Schuster, 2010, p. 435). According to the recommendations of model fit evaluation of Kline (2011, pp. 193-214), the following fit measures are used: the likelihood ratio statistic, the comparative fit index (CFI), the root mean square error of approximation (RMSEA), and the standardized root mean square residual (SRMR). Furthermore, the standardized residual covariance matrices are inspected to uncover potential misspecifications of the models in more detail. If the likelihood ratio statistic, the CFI, the RMSEA, or the SRMR indicate insufficient model fit, the model should not be retained. Instead, the possibility of re-specification should be considered (Kline, 2011, 94, 210). Starting from diagnostic statistics, re-specification has to be well justified by rational considerations and has to be clearly defined. If re-specification cannot be justified by rational considerations, no model must be retained.

Following the principle of parsimony, the statistically simplest path model is first specified in this thesis, the single-group path model. If the model fit is not adequate, the 
complexity of the model is increased due to further considerations for a more adequate specification.

The likelihood ratio statistic measures the discrepancy between the population covariance matrix and the covariance matrix predicted by the model (Kline, 2011, p. 199). As the likelihood ratio statistic follows a chi-square distribution it is also called likelihood ratio chi-square statistic (compare Kline, 2011, p. 199), abbreviated in the following as chi-square statistic:

$$
F_{M L}(N-1) \sim \chi_{d f_{M}}^{2}
$$

$F_{M L}$ is the value of the statistical criterion minimized in maximum likelihood (ML) estimation, that is, the function value of the ML discrepancy function. $\mathrm{N}$ is the sample size. As can be seen from the formula, the chi-square statistic is sensitive to sample size. The chi-square statistic evaluates if the discrepancies between the population covariance matrix and the covariance matrix predicted by the model are significant ( $p$-value $<0.05$ ). Hence, a p-value higher than 0.05 indicates good model fit. Discrepancies become significant when they exceed the level expected by sampling error (Kline, 2011, p. 200).

The CFI compares the specified model with the baseline model and is, in contrast to the chi-square statistic, not sensitive to sample size. The baseline model is a worst-case model that estimates variances of the variables only. No covariances are estimated (Kline, 2011, p. 196). Models to which applies $\chi^{2} \leq d f_{M}$ have a CFI of 1.0. For models where $\chi^{2}>\mathrm{df}_{\mathrm{M}}$, the CFI is computed according to the following formula (Kline, 2011, p. 208):

$$
C F I=1-\frac{\chi_{M}^{2}-d f_{M}}{\chi_{B}^{2}-d f_{B}}
$$

$\mathrm{df}_{\mathrm{M}}$ are the degrees of freedom of the specified model, $\chi_{B}{ }^{2}$ is the chi-square of the baseline model, $\mathrm{df}_{\mathrm{B}}$ its degrees of freedom. The CFI assesses how much the model fit of the specified model is increased compared to the baseline model (Kline, 2011, p. 208). Values of at least 0.95 indicate a relatively good model fit (Hu \& Bentler, 1999). 
Just like the chi-square statistic, the RMSEA measures the deviation of the covariance matrix of the specified model from the population covariance matrix. However, the RMSEA penalizes complex models (Kline, 2011, p. 205): ${ }^{29}$

$$
R M S E A=\sqrt{\frac{\chi_{M-d f_{M}}^{2}}{d f_{M}(N-1)}}
$$

where RMSEA = 0 if the term under the square root is negative (Kline, 2011, p. 205). A value of not higher than 0.06 is considered to indicate a good model fit (Hu \& Bentler, 1999). Besides the point estimate of the RMSEA, the $90 \%$ confidence interval is reported. The lower bound of the confidence interval should be close to 0 while the upper bound should not exceed 0.08 (Hooper, Coughlan, \& Mullen, 2008, p. 54).

The SRMR is approximately the average difference between the observed and the predicted (residual) correlations (Kline, 2011, p. 209). It should not exceed a value of 0.08 (Hu \& Bentler, 1999). The SRMR is computed according to the following formula (Hu \& Bentler, 1999, p. 3):

$$
S R M R=\sqrt{\frac{2 \sum_{i=1}^{p} \sum_{j=1}^{i}\left(\frac{s_{i j}-\widehat{\sigma}_{i j}}{s_{i i} s_{j j}}\right)^{2}}{p(p+1)}}
$$

where $s_{\mathrm{ij}}$ are the observed covariances, $\hat{\sigma}_{i j}$ are the reproduced covariances, $\mathrm{s}_{\mathrm{ii}}$ and $\mathrm{s}_{\mathrm{jj}}$ are the observed standard deviations, and $\mathrm{p}$ is the number of observed variables.

To evaluate model misfit by residuals, the standardized residual covariances matrix has to be examined. It shows the differences between the observed and the predicted covariances. The values in this matrix can be interpreted as z-scores. Values higher than 1.96 or below -1.96 indicate significant deviations of the covariance predicted by the model from the empirical covariance at a 0.05 level (Kline, 2011, 34, 209). If the values

\footnotetext{
${ }^{29}$ Simplified formula
} 
are lower than -1.96 the model overestimates the covariances. If the values are higher than 1.96 the model underestimates the covariances.

To assess how well the theories explain social inequalities in academic achievement, it is proceeded as follows. For each theory, the respective path model has to show an adequate model fit by looking at the chi-square statistic, CFI, RMSEA, SRMR, and the residual correlation matrices. If the model does not match the data, the possibility of re-specification of the model is assessed. This assessment and, if applicable, re-specification are based on rational considerations, in which statistical diagnostics are included. If the model fits the data, hypotheses can be tested by checking the path coefficients and the correlations specified in the model.

Additionally, it is evaluated how useful the theories are in terms of the explanatory power regarding the effect of social origin on academic achievement. This can be done by mediation analyses (for example MacKinnon, Fairchild, \& Fritz, 2007). For this purpose, the results of the path analyses are used. The direct effect $\left(c^{\prime} \text {, Figure } 3\right)^{30}$ is the effect of social origin on academic achievement when the mediator variable is included in the model. The indirect effect can be calculated by multiplying the path coefficients $a$, the effect of social origin on the mediator, and $b$, the effect of the mediator on academic achievement, that is ab.

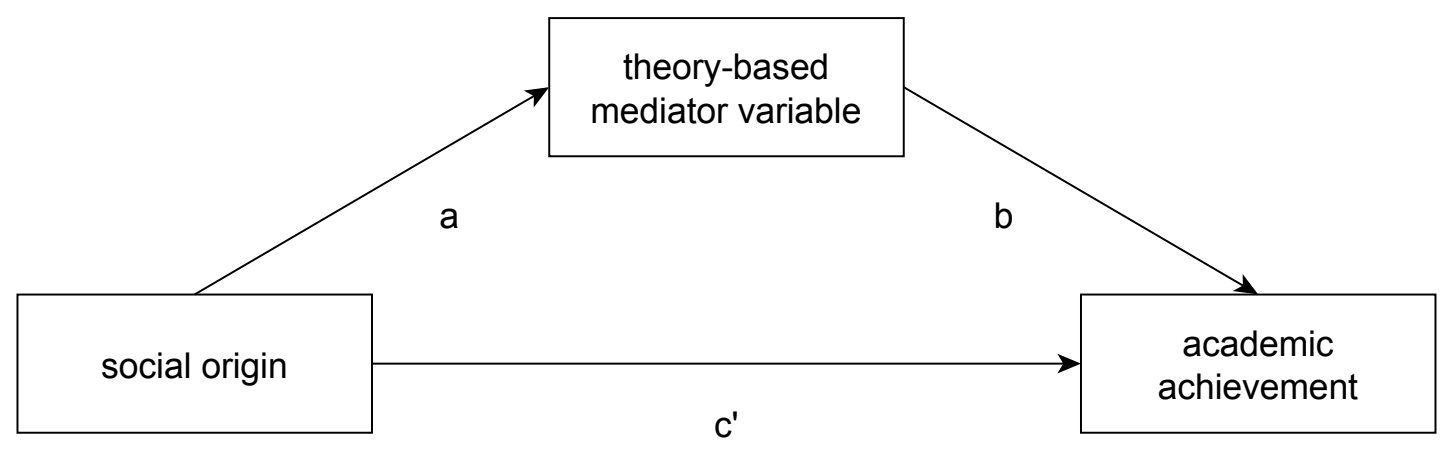

Figure 3: Direct and Indirect Effect

\footnotetext{
${ }^{30}$ For clarity, a single-mediator model is shown. The common and the revised model outlined in this thesis are multiple mediator models. However, multiple mediator models are simple extensions of singlemediator models (MacKinnon, Fairchild, \& Fritz, 2007).
} 
The sum of the direct and the indirect effect is the total effect c (Figure 4).

$$
c=c^{\prime}+a b
$$

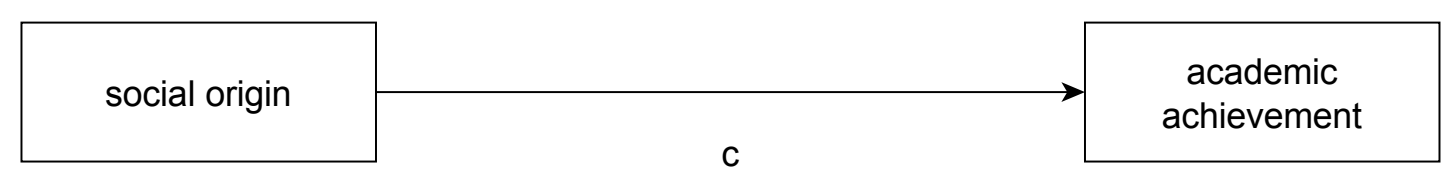

Figure 4: Total Effect

To assess the explanatory power regarding of the concurrent theories, standardized indirect effects are compared (M. W.-L. Cheung, 2009; MacKinnon et al., 2007). To achieve a better comparability of the models, the total standardized indirect effects are compared, that is, the sum of the standardized effects. (Kline, 2011, p. 167). The model with the bigger total standardized indirect effect should be preferred (for the unstandardized estimates, see Table 26).

Regression coefficients of the direct effects are standardized applying the following formula:

$$
\text { beta }=b \frac{S D_{X}}{S D_{Y}}
$$

where $b$ is the unstandardized regression coefficient, $\mathrm{SD}_{\mathrm{X}}$ is the standard deviation of $\mathrm{X}$ and $\mathrm{SD}_{\mathrm{Y}}$ is the standard deviation of Y. Standard deviations of the mediator variables do not have to be considered (compare M. W.-L. Cheung, 2009, p. 428). If X is binary, the formula is modified in the following way:

$$
\text { beta }=\frac{b}{S D_{Y}}
$$

The indirect effects of parental education on the mediator variables and on the students' average grade are standardized as follows (compare M. W.-L. Cheung, 2009, pp. 427428): 


$$
i e_{\text {standardized }}=\frac{a b}{S D_{Y}}
$$

ie $_{\text {standardized }}$ is the standardized indirect effect and $a b$ is the unstandardized indirect effect.

The standardized total effects are obtained by the formula:

$$
c_{\text {standardized }}=\frac{c^{\prime}+a b}{S D_{Y}}
$$

The corresponding standardized standard errors $\mathrm{SE}_{\text {standardized }}$ are calculated by (M. W.-L. Cheung, 2009, p. 428)

$$
S E_{\text {standardized }}=S E_{\text {unstandardized }} \frac{S D_{X}}{S D_{Y}}
$$

respectively

$$
S E_{\text {standardized }}=\frac{S E_{\text {unstandardized }}}{S D_{Y}}
$$

The 95\% Wald confidence intervals of the standardized indirect effects $C I_{i e_{\text {standardized }}}$ and total effects $C I_{c_{\text {standardized }}}$ are calculated with the following formulas (M. W.-L. Cheung, 2009, p. 428):

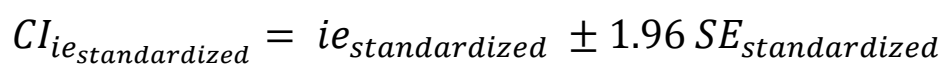

respectively

$$
C I_{c_{\text {standardized }}}=c_{\text {standardized }} \pm 1.96 S E_{\text {standardized }}
$$

Covariances $\operatorname{cov}_{\mathrm{XY}}$ are reported in standardized form (correlation cor $\mathrm{XY}$ ) according to the following formula:

$$
\operatorname{cor}_{X Y}=\frac{\operatorname{cov}_{X Y}}{S D_{X} S D_{Y}}
$$


The path models are fitted on the 100 imputed data sets to account for item-nonresponse (see chapter 3.3). Moreover, sampling strata and clustering in schools are considered in the model (compare chapter 3.1) using the R package lavaan.survey (Oberski, 2016). The procedure implemented by Oberski (2016, p. 9) gives Maximum Likelihood estimates based on the asymptotic covariance matrix derived from the observed data. Model parameters are estimated in such a way that the probability for the observed covariances is maximized. Furthermore, the package provides standard errors and chisquare-derived fit measures that are corrected for a complex sampling design and allows fitting structural equation models on multiple imputed data sets. Moreover, it reports a corrected chi-square test (Satorra-Bentler statistic) that controls for non-normality (Oberski, 2016, p. 9). Standard errors of the indirect effects are estimated according to Sobel (1982).

Additionally, another measure of the explanatory power of the concurrent models with regard to the social inequalities in students' average grade is applied. It is examined how much of the variance of the students' average grade explained by parental education remains unexplained by the mediator variables of the respective model. This is the proportion of the variance that is explained uniquely by parental education when mediator variables are considered. That is equivalent to the squared semi-partial correlation of students' average grade and parental education (compare Cohen, Cohen, West, \& Aiken, 2015, pp. 72-73). For simplicity, the Venn diagram in Figure 5 illustrates this for a model with one mediator variable. The circles represent the variances of the variables students' average grade $Y$, parental education $X$, and a mediator $M$. The total overlap between $X$ and $Y(a+c)$ is the proportion of the variance of $Y$ explained by $X$, that is the squared correlation of $X$ and $Y r_{X Y}{ }^{2}$ (compare Cohen et al., 2015, p. 38). The squared semi-partial correlation of $Y$ and $X\left(s x^{2}\right)$ is the overlap exclusively between $Y$ and $X(a)$. Since the two competing models are compared using the same data, the squared correlation $\mathrm{a}+\mathrm{c}$ is equal in the analyses of both models. Hence, the smaller the squared semi-partial correlation $\mathrm{a}$, the higher $\mathrm{c}$. 


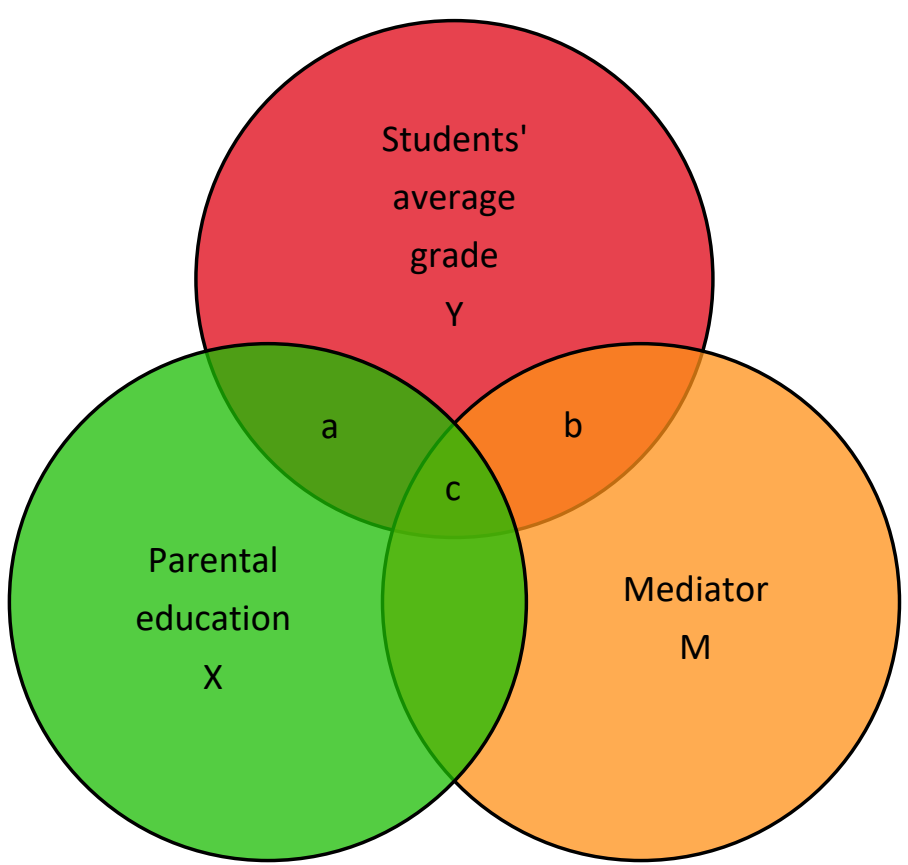

Figure 5: Venn diagram to illustrate the squared semi-partial correlation, based on Cohen et al. (2015, p. 72)

Following Cohen et al. (2015, p. 72), the squared semi-partial correlation a is computed as:

$$
a=s r_{X}^{2}=R_{Y . X M}^{2}-r_{Y M}^{2}
$$

$\mathrm{RY}_{\mathrm{X} X \mathrm{M}^{2}}$ is the proportion of the variance of $Y$ explained by $X$ and $M$, whereas $\mathrm{rYM}_{Y}{ }^{2}$ is the squared correlation of $\mathrm{Y}$ and $\mathrm{M}$. The latter can be interpreted as the proportion of the variance of $Y$ explained by $M$ (Cohen et al., 2015, p. 38). In this thesis, $s_{x}^{2}$ for the common and the revised model are computed according to this formula. The R-square ${ }^{31}$ from the regression of grades on the mediators ${ }^{32}$ are subtracted from the R-square from the regression of grades on parental education and the mediator variables.

\subsection{Path analyses}

In the following chapter first, the specifications of the path models are presented. As described in chapter 3.4, following the principle of parsimony, the statistically simplest

\footnotetext{
${ }^{31}$ For better readability, all forms of $\mathrm{R}^{2}$ in the formulas are referred to as $\mathrm{R}$-square in the continuous text.

32 The mediator variables of the common model are parents' and students' high cultural participation as well as reading activities (see Figure 6 respectively Figure 8). The mediator variables of the revised model are parents' and students' high cultural practices as well as reading comprehension and orthographic competence (see Figure 7 respectively Figure 9).
} 
path model is first specified in this thesis, the single-group path model. If the model fit is not adequate, the complexity of the model is increased due to further considerations for a more adequate specification. Second, the statistical power and, third, the model fit evaluations, which are used as decision criteria for the model specification, are described. The model fit indicators have been presented in chapter 3.4. After choosing the adequate model specification, the results of the empirical analyses of the chosen path models, that are multiple-group path models, are presented and compared for the common and the revised model.

\subsubsection{Model specification - single-group path analyses}

According to the theories outlined in chapters 2.4.1 and 2.4.2 and the operationalizations described in chapter 3.2, the path models for the common and the revised theory are specified as shown in Figure 6 and Figure 7. Variables are displayed as rectangles. The variables above the dashed line refer to the parents, below to the students. Social origin, measured as highest education of the parents (green) is at the top, and academic achievement, measured as students' average grade (red) is at the bottom. The cultural capital variables that mediate the effect of social origin on academic achievement are in between (orange, lighter blue, darker blue). Besides a label, all variables also have a numbering, starting with highest education of the parents $(x 1)$ to average grade $(x 9)$. In order to make the figures clearer, the control variables are included as comments in the rectangles. The control variables are abbreviated with $\mathrm{c}$ and a consecutive number, for example, the migration background has the abbreviation $\mathrm{c} 1$. The key for these abbreviations is at the bottom to the right of the figures. Straight one-headed arrows represent the path coefficients. They are named by the prefix $p$, the number of the variable they point to, and the number of the variable they start. For example, the path running from highest education of the parents $(x 1)$ to average grade $(x 9)$ is labeled p91. Curved twoheaded arrows represent residual correlation coefficients, that is, correlations of two variables net of the influences of common explaining variables. Their labels include the prefix $r$ and the numbers of the concerned variables. The residual variances respectively the error terms are shown as arrows pointing to the variables, labeled by a letter. 


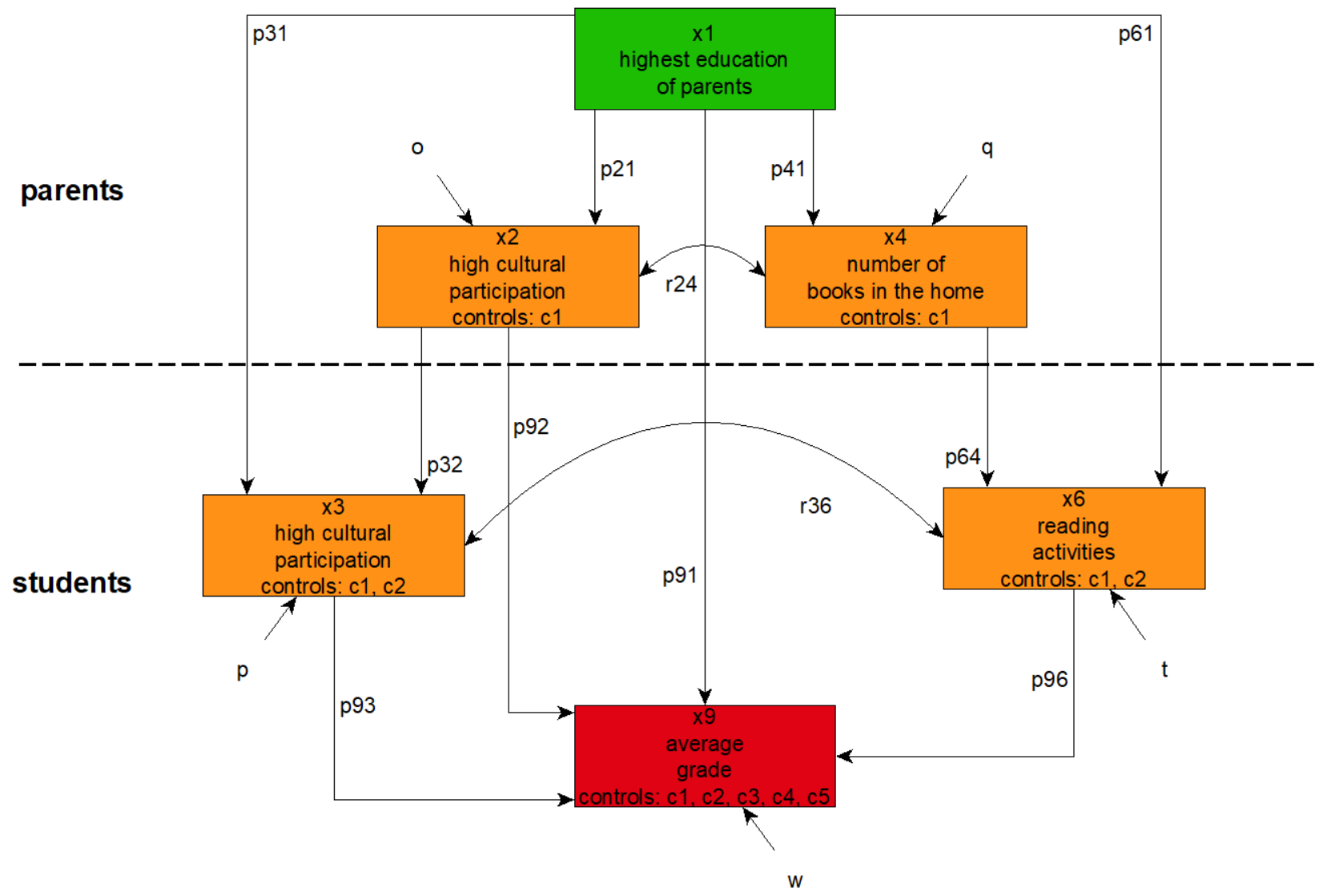

key:
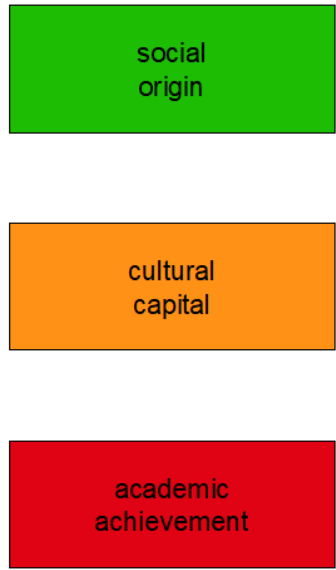

controls:

c1: migration background;

c2: sex; c3: perceptual speed;

c4: reasoning

c5: type of school

Figure 6: Specification of the common cultural capital model - single-group path analysis 


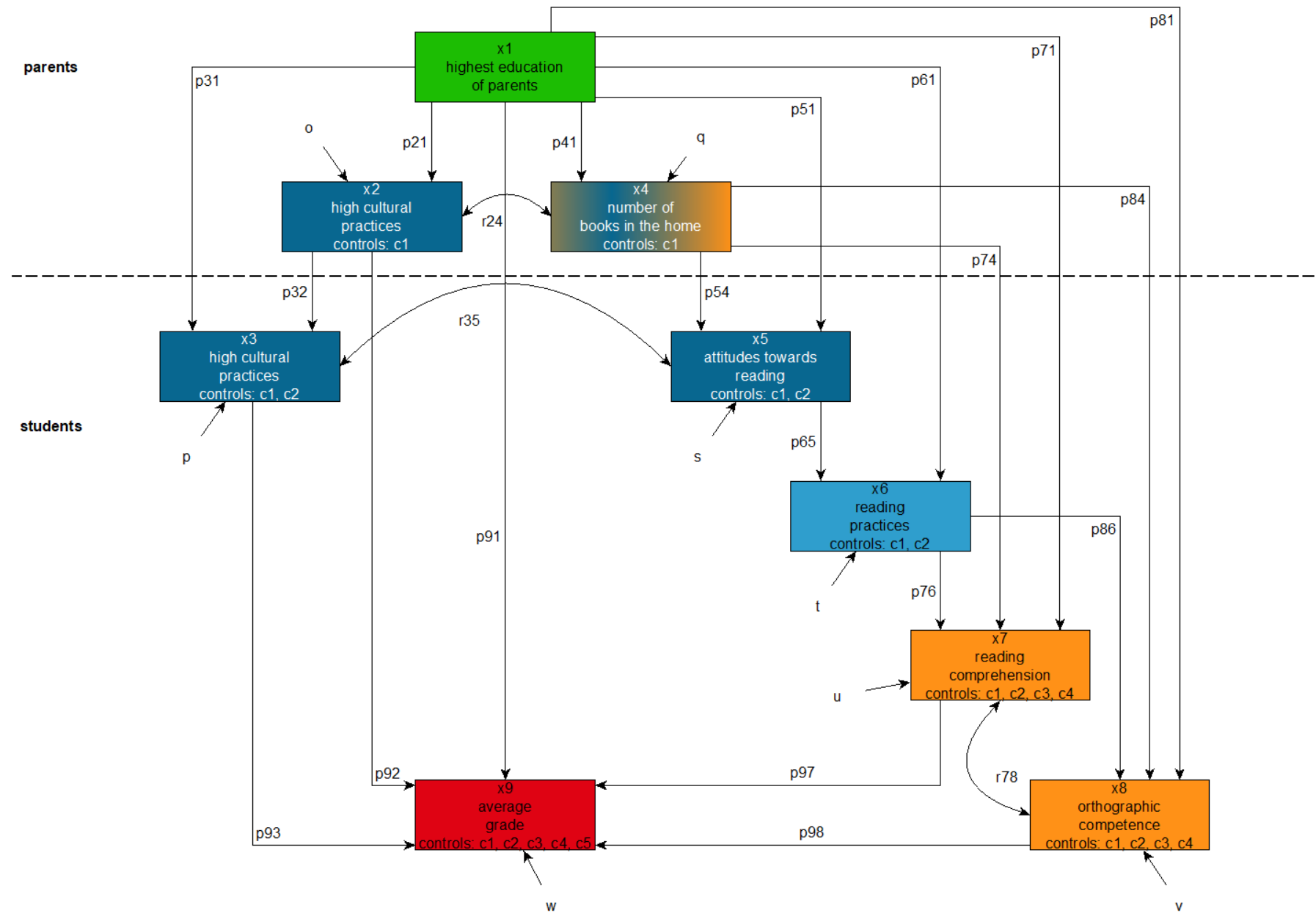

key:

social
origin

habitus

practice

cultural
capital

habitus
$\&$

$\stackrel{2}{\text { cultural capital }}$

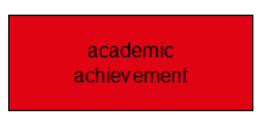

controls:

c1: migration background;

c2. sex, c3. perceptual speed
c4 reasoning

c5. type of school 


\subsubsection{Sample size and statistical power - single-group path analyses}

It is checked if the sample size is large enough to retain valid estimates. As a rule of thumb, the ratio of sample size $(N)$ to the number of free parameters to be estimated (q) should be at least 10 (Kline, 2011, pp. 11-12). The revised model contains 76 free parameters and the sample size is 4,924 . Hence, the $\mathrm{N}: \mathrm{q}$ ratio is 65 . For the common model, there are 44 free parameters and the same sample size resulting in an $\mathrm{N}$ :q ratio of 112. According to this rule, sample size is sufficient to estimate both models.

Moreover, it is analyzed if the statistical power is sufficient to detect significant differences between the predicted covariance matrix and the observed one by the RMSEA (Kline, 2011, pp. 222-225). According to the method provided by MacCallum et al. (1996), statistical power can be calculated by using the degrees of freedom of the model, the sample size, and the significance level $\alpha$. The statistical power is estimated for the close-fit test with the R function findRMSEApower of the SEMTools package (Jorgensen, 2019). According to MacCallum et al. (MacCallum et al., 1996, p. 138), this gives the statistical power for testing mediocre $\left(\varepsilon_{\mathrm{a}}: \mathrm{RMSEA} \geq 0.08\right)$ versus good or close fit $\left(\varepsilon_{0}:\right.$ RMSEA $\leq 0.05)$. Values of at least 0.8 indicate sufficient statistical power. Hence, the statistical power of the revised model is calculated with 40 degrees of freedom (see Table 7), $N=$ $4,924, \alpha=0.05, \varepsilon_{0}:$ RMSEA $=0.05$, and $\varepsilon_{a}:$ RMSEA $=0.08$. For computing the statistical power of the common model, the same values are used except for the degrees of freedom that equal to 21 (see Table 7). For both models, power is 1 meaning that sample size is large enough to differentiate between a mediocre and a good fitting model. ${ }^{33}$

\subsubsection{Model fit evaluation - single-group path analyses}

Table 7 shows the model fit indices for the common and the revised model. According to these indices, the revised model (1) fails the threshold of the chi-square statistic $(p=$ 0.000 ), shows (2) an insufficient RMSEA value (RMSEA $=0.066)$, and ( 3 ) an insufficient $\mathrm{CFI}$ value $(\mathrm{CFI}=0.925)$. That means that $(1)$ discrepancies between the covariance matrix of the revised model and the observed covariance matrix are significant, (2) these dis-

\footnotetext{
${ }^{33} 1$ is the maximum value to be reached.
} 
crepancies are considered as too high after correcting for parsimony, and (3) the improvement of the global model fit of the specified model compared to the base model is not high enough (compare chapter 3.4). In contrast, the common model exhibits a good model fit. The corresponding values are all within the specified limits. In order to uncover the causes of the insufficient model fit of the revised model, an investigation of the standardized residual covariances is necessary.

Table 7: Model fit indices of the single-group path analyses

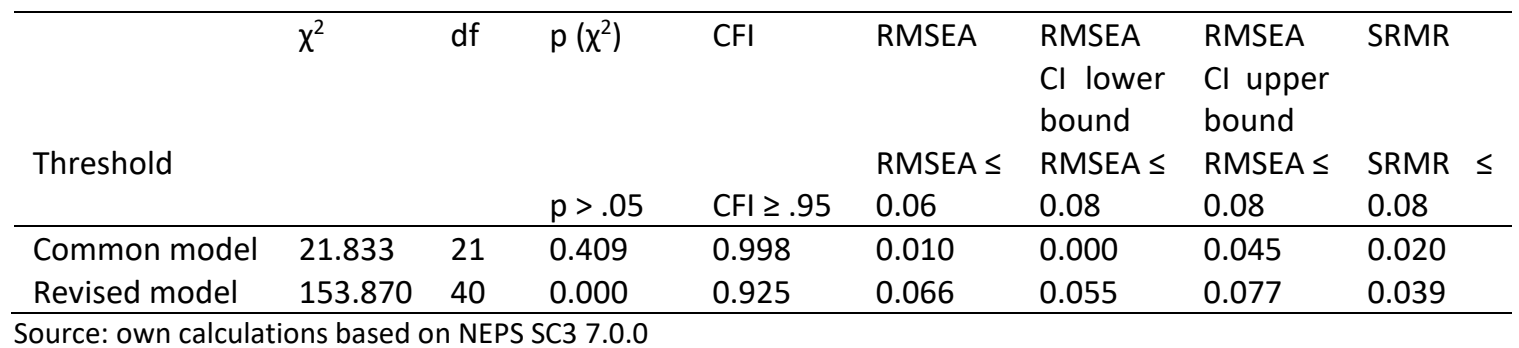

\section{Standardized residual covariances}

Table 15 (Appendix) and Table 16 (Appendix) show the standardized residual matrices for the common and the revised model. To save space, the abbreviations from Figure 6 and Figure 7 are used for the row and column labels. As described in chapter 3.2, the categorical variables parental education (x1) and type of school (c5) are transformed in a set of dummy variables. To designate the corresponding dummy variables in the tables, an extension introduced by a dot is used (for example $\times 1.2$ for intermediate secondary education of the parents). Significant values are highlighted in bold. In the following, not every significant residual covariance is reported. Rather, those patterns are described that seem to matter most regarding the model fit.

Regarding the revised model, significant differences between the observed and the predicted values primarily occur regarding the variables attitudes towards reading (x5), average grade (x9), reasoning (c4) and attended type of school (c5.2: Gymnasim; c5.3: school without tracking). In contrast to the predicted data, the observed data show covariances between attitudes towards reading and the linguistic skills variables. This finding is in accordance with the study of McElvany et al. (2008, see chapter 2.2.1). They find 
that prior reading competence has a positive effect on attitudes towards reading. This path is not specified in the revised model, as it would imply a feedback loop. In crosssectional analyses, like the current one, feedback loops might cause serious problems (Kline, 2011). Additionally, observed and predicted covariances differ with regard to the average grade. The correlations of grades with students' high cultural practices, reading comprehension, and orthographic competence are overestimated by the revised model. Differential effects of the explanatory variables across the types of school on the grades may play a role (Autorengruppe Bildungsberichterstattung, 2016). Moreover, the model does not take into account correlations between the control variable reasoning and the mediating variables parents' high cultural practices, number of books in the home, attitudes towards reading, and reading practices. The variable reasoning is not specified as endogenous $^{34}$ as it is a control variable in the regression equation estimating school grades, only. The dependencies of auxiliary variables are not fully taken into account by the model. This is accepted as a necessary occurrence for the empirical testing of the theory. Moreover, differences between the observed covariances and the predicted ones occur considering the variables attitudes towards reading $(x 5)$, reading comprehension (x7) and orthographic competence (x8). Furthermore, covariances between school grades (x9) and linguistic competencies are underestimated by the model as the negative values indicate.

The residual covariance matrix of the common model shows essentially the same patterns with regard to the variables reasoning and type of school.

Apparently, the distributions of the variables representing habitus, practice, and cultural capital (revised model) respectively cultural capital (common model) differ strongly by type of school. From a theoretical perspective, this can be interpreted as an effect of tracking itself: Students with characteristics that have a positive effect on academic achievement are sorted into the higher school track, while students with less positive

\footnotetext{
${ }^{34}$ Endogenous variables are explained by the model.
} 
characteristics are sorted into the lower or intermediate school track. Students attending schools with no tracking either attend a primary school or an orientation stage. Hence, they are not sorted according to their characteristics yet or they attend a type of school that has no external tracking. Thus, it can be assumed that students at these types of schools show characteristics that are in between the lower and intermediate track and the higher track. Additionally, it seems likely that the standard deviations at the higher track are smaller than at other types of school. This is because students attending the higher track are selected more strictly according to the mediating variables.

Table 8 shows the means and the standard deviations for the mediating variables. The rank order of the means assumed above applies to all the variables. Additionally, a oneway analyses of variance (ANOVA) is conducted to test if the observed differences in the means are statistically significant. The resulting $p$-values are reported in Table 8. Indeed, all the differences are highly significant $(p=0.000)$. To assess if the assumption of higher group homogeneity due to sorting into tracks, standard deviations across the types of school are considered. Except for parents' high cultural participation, standard deviations are smallest in the higher tracks. Regarding parents' high cultural participation, standard deviation is smallest for the lower and intermediate track. However, some differences between the standard deviations are small. A Levene test, by Levene (1960), is conducted to show whether the variances significantly differ across the groups. They do at a 0.05-level except for students' high cultural participation and time spent on reading. In sum, all means and most standard deviations of the variables differ considerably by type of school. Hence, there are strong indications for re-specifying the revised and the common model to avoid misfit and potentially biased estimates. 
Table 8: Means and standard deviations of the mediating variables by type of school

\begin{tabular}{|c|c|c|c|c|c|c|c|}
\hline & & Obs & Mean & SD & $\begin{array}{l}\text { p-value } \\
\text { (ANOVA) }\end{array}$ & $\begin{array}{l}\text { p-value } \\
\text { (Levene Test) }\end{array}$ & $\mathrm{R}^{2}$ \\
\hline Books in the home & $\begin{array}{l}\text { Lower and intermediate } \\
\text { track } \\
\text { Schools without tracking } \\
\text { Higher track }\end{array}$ & $\begin{array}{l}1,264 \\
476 \\
1,740\end{array}$ & $\begin{array}{l}3.66 \\
4.13 \\
4.51\end{array}$ & $\begin{array}{l}1.26 \\
1.27 \\
1.16\end{array}$ & 0.000 & 0.033 & 0.10 \\
\hline $\begin{array}{l}\text { High cultural participation } \\
\text { (parent) }\end{array}$ & $\begin{array}{l}\text { Lower and intermediate } \\
\text { track } \\
\text { Schools without tracking } \\
\text { Higher track }\end{array}$ & $\begin{array}{l}1,264 \\
476 \\
1,742\end{array}$ & $\begin{array}{l}5.18 \\
6.25 \\
6.92\end{array}$ & $\begin{array}{l}2.13 \\
2.66 \\
2.72\end{array}$ & 0.000 & 0.000 & 0.09 \\
\hline $\begin{array}{l}\text { High cultural participation } \\
\text { (student) }\end{array}$ & $\begin{array}{l}\text { Lower and intermediate } \\
\text { track } \\
\text { Schools without tracking } \\
\text { Higher track }\end{array}$ & $\begin{array}{l}1,559 \\
551 \\
2,054\end{array}$ & $\begin{array}{l}5.70 \\
6.35 \\
6.43\end{array}$ & $\begin{array}{l}2.46 \\
2.50 \\
2.43\end{array}$ & 0.000 & 0.649 & 0.02 \\
\hline Time spent on reading & $\begin{array}{l}\text { Lower and intermediate } \\
\text { track } \\
\text { Schools without tracking } \\
\text { Higher track }\end{array}$ & $\begin{array}{l}1,617 \\
567 \\
2,088\end{array}$ & $\begin{array}{l}0.74 \\
0.86 \\
0.97\end{array}$ & $\begin{array}{l}0.64 \\
0.64 \\
0.62\end{array}$ & 0.000 & 0.136 & 0.03 \\
\hline Attitudes towards reading & $\begin{array}{l}\text { Lower and intermediate } \\
\text { track } \\
\text { Schools without tracking } \\
\text { Higher track }\end{array}$ & $\begin{array}{l}1,747 \\
603 \\
2,133\end{array}$ & $\begin{array}{l}8.45 \\
9.02 \\
9.86\end{array}$ & $\begin{array}{l}2.97 \\
2.80 \\
2.36\end{array}$ & 0.000 & 0.000 & 0.06 \\
\hline Reading comprehension & $\begin{array}{l}\text { Lower and intermediate } \\
\text { track } \\
\text { Schools without tracking } \\
\text { Higher track }\end{array}$ & $\begin{array}{l}1,976 \\
672 \\
2,261\end{array}$ & $\begin{array}{l}-0.52 \\
-0.25 \\
0.57\end{array}$ & $\begin{array}{l}1.12 \\
1.27 \\
1.11\end{array}$ & 0.000 & 0.000 & 0.17 \\
\hline Orthographic competence & $\begin{array}{l}\text { Lower and intermediate } \\
\text { track } \\
\text { Schools without tracking } \\
\text { Higher track }\end{array}$ & $\begin{array}{l}1,982 \\
677 \\
2,265\end{array}$ & $\begin{array}{l}-0.57 \\
-0.40 \\
0.70\end{array}$ & $\begin{array}{l}1.26 \\
1.31 \\
1.13\end{array}$ & 0.000 & 0.000 & 0.21 \\
\hline
\end{tabular}

Source: own calculations based on NEPS SC3 7.0.0

\subsubsection{Model re-specification - multiple-group path analyses}

As shown above, it is recommended to re-specify the model. One possible way of accounting for the relations of type of school and the mediating variables is to include the variable type of school in the each of the regression equations. This approach, however, leads to a clear underestimation of the effect of parental education. Each of the endogenous variables as well as the variable type of school are related to parental education. Including the variable type of school into the regression equations causes a reduction of the effect of parental education because of these variances shared by education and 
type of school. However, this effect is important for answering the research question. Moreover, it is not plausible that the type of school has a causal effect on the endogenous variables. In general, the type of school affects the linguistic skills and, possibly, students' high cultural practices. However, this effect is likely to be negligible at the time of the survey, as students have only been attending secondary school for a few months. The influence of the parents begins at a very early age (compare chapter 2.3.1). Hence, the endogenous variables and the variable type of school correlate as they are both affected by parental education. Additionally, there is a causal effect of linguistic skills on the type of school that is mediated by grades (compare Bos et al., 2012, p. 224). Students are sorted to school tracks according to their skills. It can be assumed that sorting is based on high cultural practices as well. Summarized, regressing the endogenous variables on the type of school cannot be recommended.

An alternative solution to account for tracking induced differences in the mediating variables is multiple-group analysis. Path analyses provide the possibility to estimate a model for multiple groups separately. Moreover, model parameters can be estimated freely for each of the groups or they can be fixed to be equal across the groups. To consider the distributional differences between the types of school, group-specific intercepts can be estimated. Thus, the path model is estimated separately for the three types of school. The intercepts of the regressions in the model are freely estimated for each of these groups whereas correlations are fixed across groups as well as most regression coefficients. Judging from the residual covariance matrices, there are differential effects of parents' and students' high cultural practices as well as students' reading comprehension and orthographic competence on students' average grade. Overall, the different types of schools have different requirements. Therefore, the determinants of grades can have different effects on grades depending on the type of school. ${ }^{35}$ Hence, all the regression coefficients for the average grade are estimated freely across the types of

\footnotetext{
${ }^{35}$ Indeed, residual covariance matrices indicate differing correlations between grades and their predictors across the types of school (see Appendix, Table 17, Table 18, Table 19). Rows and columns of x9 (grades) contain a number of significant values.
} 
school. The other regression coefficients are fixed across the groups to be equal. It is assumed that the mechanisms explaining the acquisition of habitus, cultural capital, and the occurrence of practices are the same in all groups.

Figure 8 and Figure 9 show the so specified path models. Group references are represented by the abbreviations not (no tracking / schools without tracking), lit (lower and intermediate track), and hit (higher track). Group-specific path coefficients and residuals are indicated by corresponding suffixes.

\subsubsection{Sample size and statistical power - multiple-group path analyses}

Using multiple-group analyses, the models are estimated for three groups separately: students attending a lower or intermediate school track, students attending a higher school track, and students attending a type of school without tracking. Thus, the data are split into three analytical samples. The sub-sample of the lower and intermediate track contains 1,982 students, the sub-sample of the higher track 2,265, and the subsample of the schools without tracking 677 (compare chapter 3.2). The revised path model contains 82 parameters to be estimated. This results in an N:q ratio of about 24 for the sub-sample of the lower and intermediate tracks, about 27 for the higher track sub-sample, and around 8 for the sub-sample of schools without tracking. This means that sample size is large enough to estimate valid parameters for two of the sub-samples $(N: q \geq 10)$, while estimates of the sub-sample for the schools without tracking have to be interpreted with caution $(\mathrm{N}: \mathrm{q}<10)$. The common path model comprises 47 free parameters. This results in an N:q ratio of about 42,48 , and 14 . Thus, sample sizes are sufficient for valid parameter estimation in the common path model in the three subsamples.

The statistical power is sufficient for RMSEA with $N=4,924, \alpha=0.05, \varepsilon_{0}: \operatorname{RMSEA}=0.05$, and $\varepsilon_{\mathrm{a}}:$ RMSEA $=0.08$ (compare chapter 3.5.2). The revised model has 276 degrees of freedom resulting in a power of 1 . Thus, power is sufficient. The common model also has sufficient power (1 with 150 degrees of freedom). 


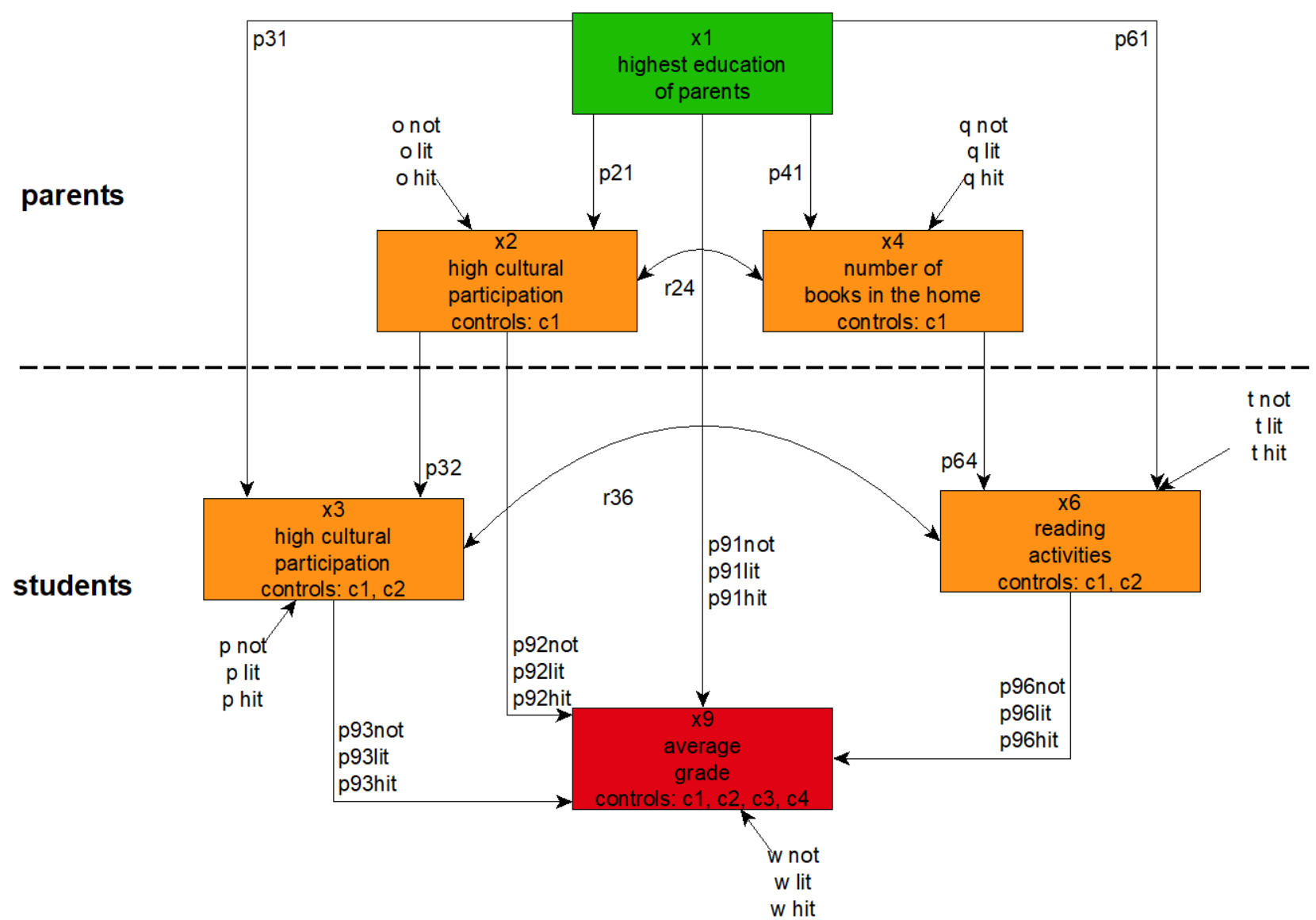

key:
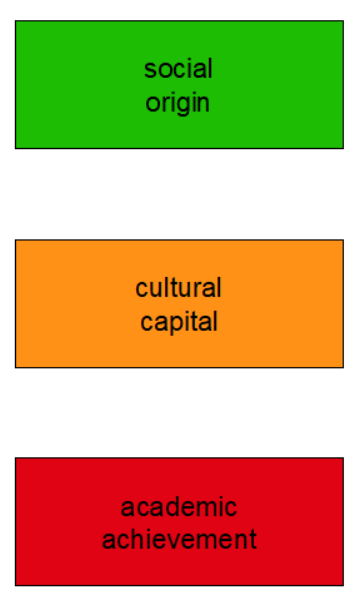

controls:

c1: migration background

c2: sex; c3: perceptual speed;

c4: reasoning

Figure 8: Specification of the common cultural capital model - multiple-group path analysis

Notes: p: path; r: correlation; not: school without tracking; lit: lower and intermediate track; hit: higher track 


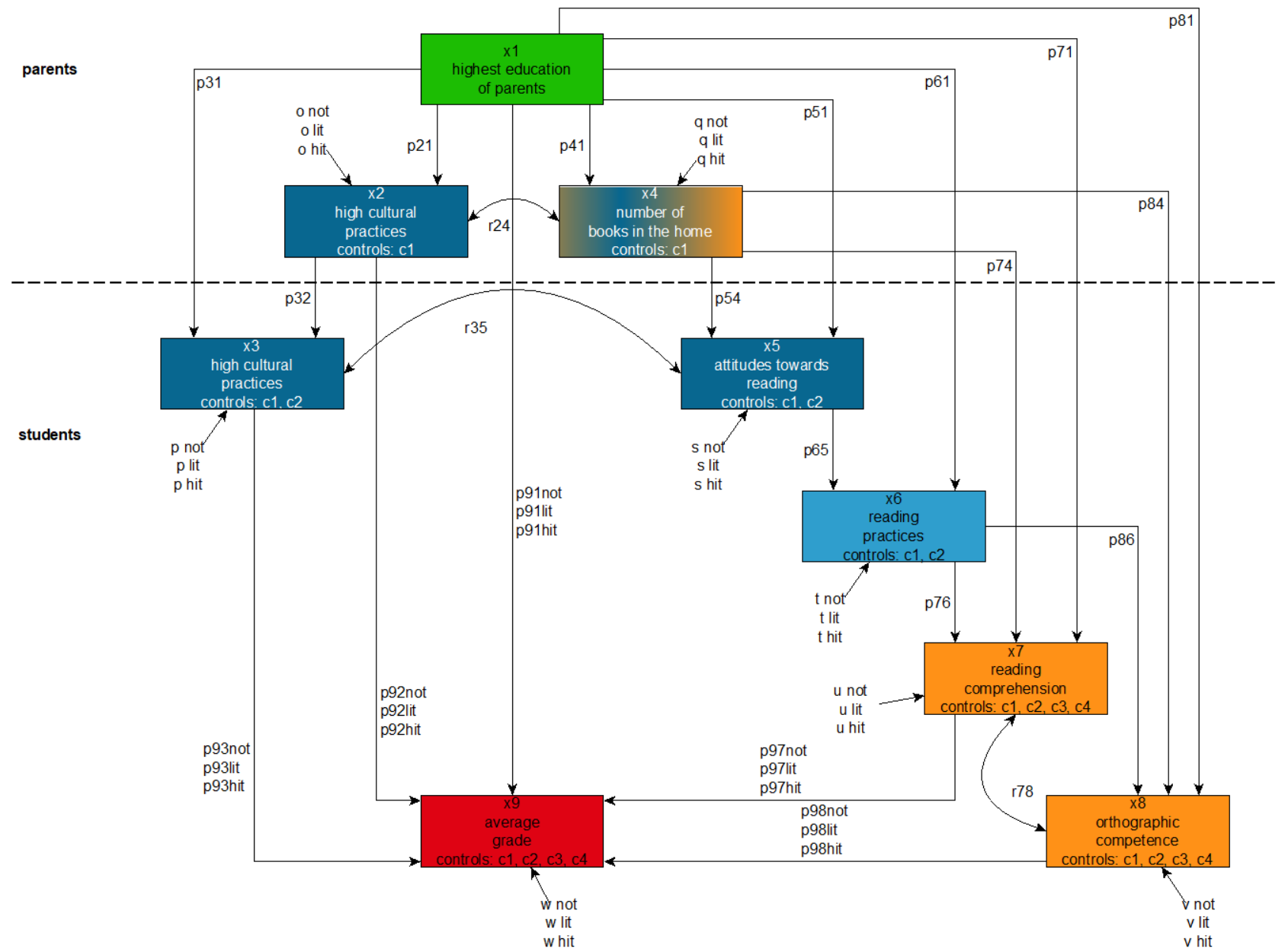

key:

practice

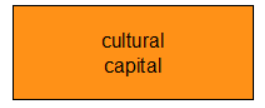

habitus

$\&$
cultural capital

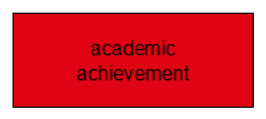

controls:

c1: migration background; c2: sex; c3: perceptual speed; c4: reasoning

9: Specification of the revised theoretical model - multiple-group analysis

Notes: p: path; r: correlation; not: school without tracking; lit: lower and intermediate track; hit: higher track 


\subsubsection{Model fit evaluation - multiple-group path analyses}

Table 9 shows the model fit indices for both models. The common and the revised model exhibit a good model fit. This applies in particular to the common model.

Table 9: Model fit indices of the multiple-group path analyses

\begin{tabular}{|c|c|c|c|c|c|c|c|c|}
\hline & $x^{2}$ & $d f$ & $p\left(\chi^{2}\right)$ & $\mathrm{CFI}$ & RMSEA & $\begin{array}{l}\text { RMSEA } \\
\mathrm{Cl} \text { lower } \\
\text { bound }\end{array}$ & $\begin{array}{l}\text { RMSEA } \\
\mathrm{Cl} \text { upper } \\
\text { bound }\end{array}$ & SRMR \\
\hline Threshold & & & $p>.05$ & $\mathrm{CFI} \geq .95$ & $\begin{array}{l}\text { RMSEA } \leq \\
0.06\end{array}$ & $\begin{array}{l}\text { RMSEA } \leq \\
0.08\end{array}$ & $\begin{array}{l}\text { RMSEA } \leq \\
0.08\end{array}$ & $\begin{array}{l}\text { SRMR } \leq \\
0.08\end{array}$ \\
\hline Common model & 46.947 & 91 & 1.000 & 1.000 & 0.000 & 0.000 & 0.000 & 0.021 \\
\hline Revised model & 197.342 & 186 & 0.271 & 0.996 & 0.010 & 0.000 & 0.021 & 0.031 \\
\hline
\end{tabular}

Source: own calculations based on NEPS SC3 7.0.0

\section{Standardized residual covariances}

Differences between observed and predicted covariances of the revised model occur regarding attitudes towards reading $(x 5)$ on the one hand and reading comprehension (x7) and orthographic competence (x8) on the other hand in every sub-sample (compare Appendix, Table 20, Table 21, Table 22). Moreover, residual covariances of parents' high cultural practices (x2) differ across the sub-samples. The same is true for students' high cultural practices (x3). However, differences between observed and model implied covariances are small so that the chi-square statistic and the standardized root mean square residual (SRMR) remain below the critical thresholds $\left(p\left(\chi^{2}\right)=0.271\right.$, SRMR $=$ 0.031; see Table 9).

Considering the common model applied to the higher track sub-sample (see Table 25), the covariance between parental intermediate secondary education (x1.2) and parents' high cultural participation (x2), students' high cultural participation ( $x 3$ ), and reading activities (x6) are overestimated. In contrast, in the sub-sample of the lower and intermediate track (see Appendix, Table 24) covariances of parental intermediate secondary education (x1.2) and parents' and students' high cultural participation $(x 2, x 3)$ are underestimated. The residual covariances of grades (x9) and cultural capital variables are inconsistent across the different sub-samples. In the higher track sub-sample, the covariance of grades $(x 9)$ and reading activities $(x 6)$ are underestimated. With regard to the 
lower and intermediate track sub-sample, the covariance of grades (x9) and students' high cultural participation (x3) are overestimated. Predicted covariances of grades (x9) and parents' high cultural participation, number of books in the home (x4), and reading activities (x6) for the sub-sample with no tracking are underestimated. Nonetheless, in sum, deviations of observed and predicted covariances are quite small judging from the chi-square statistic and the SRMR $\left(p\left(\chi^{2}\right)=1.000\right.$, SRMR $=0.021$; see Table 9).

Overall, the revised and the common model fit quite well when specified as described in chapter 3.5.4. Following, selected hypotheses described in chapter 2.4 are tested.

\subsubsection{Empirical results}

Since the statistics to evaluate the model fit speak for the use of the model, the results can now be interpreted. The path diagrams (Figure 10 and Figure 13) contain the results of all groups of the multiple-group analysis. Estimates that are freely estimated across groups (regression coefficients, intercepts, and residuals) are preceded by the abbreviation not (no tracking), lit (lower and intermediate track) and hit (higher track). Estimates that are constrained to be equal across groups (regression coefficients and correlations) are reported without abbreviation. As parental education is introduced as a set of four dummy variables into the model (intermediate secondary, higher secondary, lower tertiary, and higher tertiary education), paths entailing that variable are described by four path coefficients. Parents with lower secondary education are defined as the reference group. Within the rectangles of the dependent variables, control variables in the respective regression equation are reported.

Figure 10 shows the results of the path analysis of the common model, whereas Figure 13 displays the finding of the revised model. As described in chapter 3.4, standardized regression coefficients are reported as effect sizes are compared. In multiple-group analysis, regression estimates are usually standardized according to the standard deviations of the respective group for which they are estimated. If standard deviations differ across the groups, the standardized coefficients do as well. This clearly applies to the current sample (compare Table 8). Hence, effect sizes are not comparable in ordinary multiplegroup analysis. To make effect sizes comparable, estimates are standardized according 
to standard deviations of the imputed data of the total sample (see Table 6). The same is true for the covariance estimates. Error variances reported are standardized with regard to the respective group. Hence, one minus the error variance equals R-square.

First, the results of the common cultural capital model are presented as this model serves as benchmark for the revised model.

\section{The common model}

In the following two sections, the results of the path model of the common model are presented. The first section deals with the results regarding the acquisition of cultural capital, the second section with the impact of cultural capital on academic achievement.

\section{The acquisition of cultural capital}

The common cultural capital theory claims that the various dimensions of cultural capital are unequally distributed across social classes. The higher the social class, the higher the endowment with cultural capital. Furthermore, cultural capital is transmitted within the family.

Results show that higher educated parents participate more often in high cultural activities (p21, Figure 10; H14.C; see also Appendix Table 26). Moreover, the path coefficient p32 indicates that students whose parents participate more frequently in high cultural activities do so as well (H10.C). Except for higher tertiary education, the direct effects of parental education on students' high cultural participation (p31) are negative. However, standard errors of these regression coefficients are large. Except for the regression coefficient of higher secondary education the resulting $p$-values are higher than 0.05 . The $p$-value of higher secondary education is 0.49 (see Table 26). Looking at the results as a whole, the direct effects of parental education cannot be declared as robust. However, the indirect effects through parents' high cultural activities are positive and significant (Figure 11; see also Appendix Table 27). This can be seen from the fact that the confidence intervals do not include the value of zero. Hence, social differences in students' 
high cultural participation are mediated by parents' high cultural activities. It can be assumed that the effect is shown by the joint participation of parents with their children. This might also explain the strength of the relation p32 (Figure 10 , beta $=0.244$ ).

Results with regard to the acquisition of reading-related cultural capital, support hypotheses of the common theory, too. The higher the education of parents, the more books families have in their homes. After controlling for migration background, this effect ranges from beta $=0.441$ ( $p 41$ : intermediate secondary education) to beta $=1.505$ (p41: higher tertiary education). Thus, it can be argued that the reading climate at the students' homes is indeed class-specific. The higher the social class of a family, the more positive the reading climate (H15.C). Moreover, the reading climate influences students' reading activities. The more books families have in their homes, the more students read (p64, H11.C). Figure 11 shows that the indirect effects of parental education on reading activities through number of books in the home are positive and significant. Thus, part of the social differences in reading activities can be explained by socially different reading climates.

Additionally, the positive and significant correlations between parents' high cultural participation and the number of books in the home $(r 24)$ and students' high cultural participation and reading activities ( $r 36$ ) also back the idea of class-specific cultural capital (H16.C and H17.C).

In sum, results suggest that upper-class parents have higher cultural capital in terms of high cultural participation and reading and that they transmit this capital to their children (see Table 10). Even though the transmission of cultural capital is a necessary condition for the explanation of the social achievement gap by cultural capital, it is not a sufficient one. Whether cultural capital has an effect on academic achievement is of equal importance. 
Table 10: Overview of the results on the acquisition of cultural capital - common theory

\begin{tabular}{llll} 
Number & Path & Hypothesis & Result \\
\hline H14.C & p21 & $\begin{array}{l}\text { The higher the social class of parents, the higher } \\
\text { the parents' high cultural knowledge. }\end{array}$ & $\checkmark$ \\
H10.C & p32 & $\begin{array}{l}\text { The more parents participate in high culture, the } \\
\text { more do their children. } \\
\text { The higher parents' social class position, the more } \\
\text { H15.C }\end{array} \quad$ p41 & $\begin{array}{l}\text { positive is the reading climate they provide at } \\
\text { home. }\end{array}$ \\
H11.C & p64 & $\begin{array}{l}\text { The more positive the reading climate at home, } \\
\text { the more children read. }\end{array}$ & $\checkmark$ \\
H16.C & r24 & $\begin{array}{l}\text { The more positive the reading climate at home, } \\
\text { the more often parents participate in high cultural } \\
\text { activities. }\end{array}$ & $\checkmark$ \\
H17.C & r36 & $\begin{array}{l}\text { The more students read, the more often they par- } \\
\text { ticipate in high cultural activities. }\end{array}$
\end{tabular}

Notes: $\checkmark$ indicates that the hypothesis is confirmed, $\mathrm{x}$ indicates that it is falsified. 


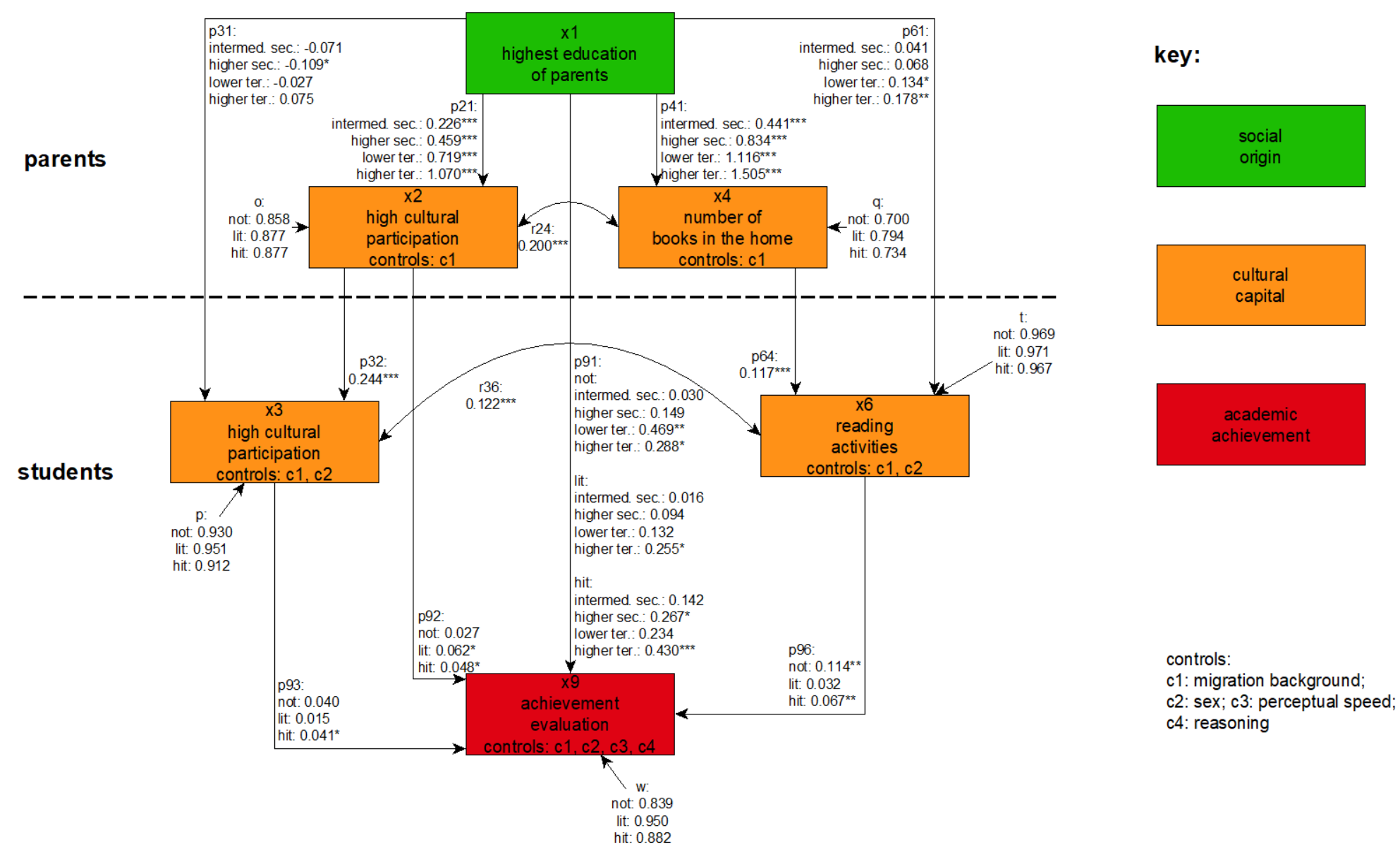

Figure 10: Path diagram: common model

Source: own calculations based on NEPS SC3 7.00

Notes: ${ }^{*} p<0.05 ;{ }^{* *} p<0.01 ;{ }^{* *} p<0.001$; standardized coefficients; not: schools without tracking; lit: lower and intermediate track; hit: higher track 
Students' high cultural practices

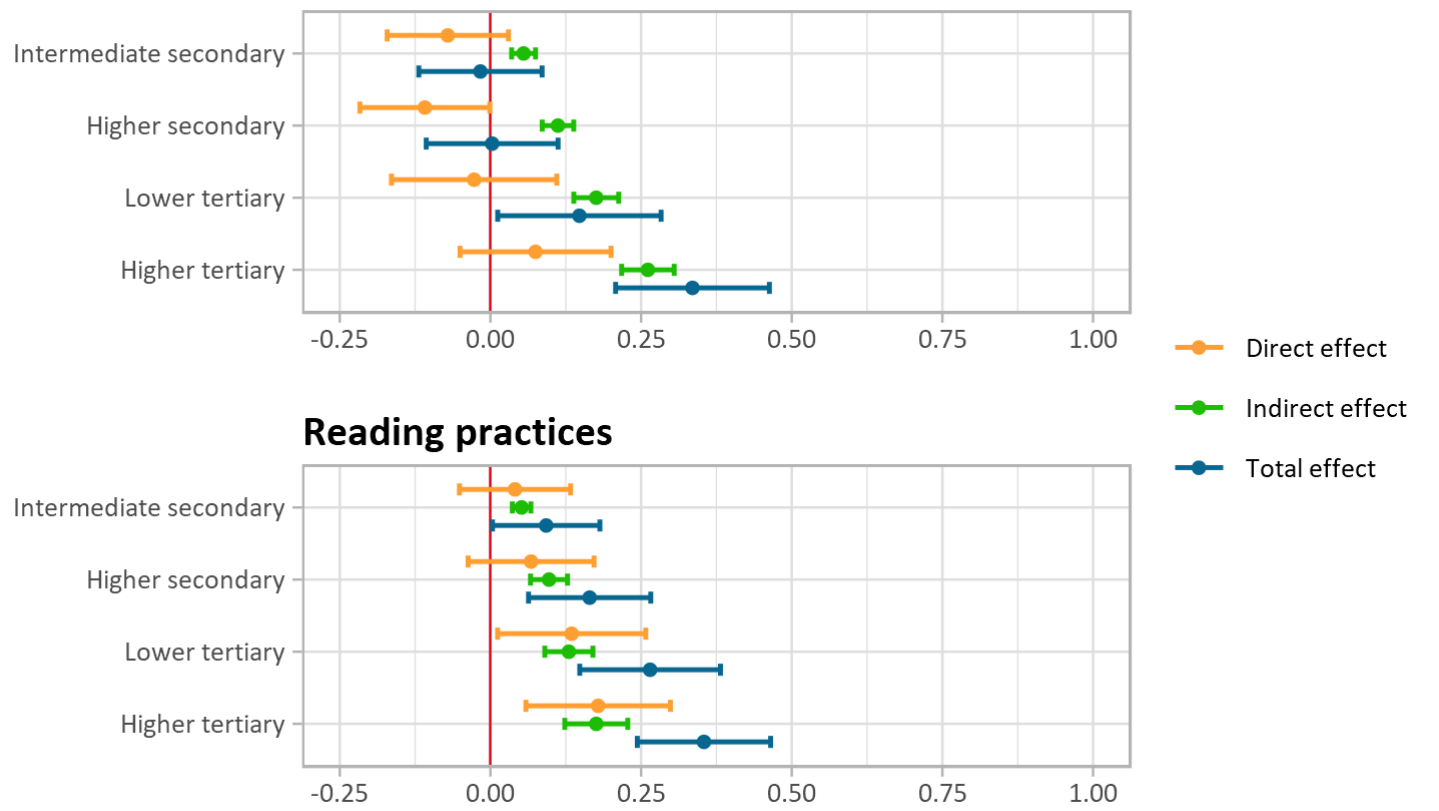

Figure 11: Direct, indirect, and total effects of parental education on mediator variables - common model Source: own calculations based on NEPS SC3 7.0.0

Note: Wald confidence intervals are calculated for $\alpha=0.05$

The impact of cultural capital on academic achievement

Findings concerning the impact of cultural capital on academic achievement are mixed. Standardized coefficients of high cultural participation are small in each school track ranging from 0.015 to 0.062 . Moreover, the effects of students' high cultural participation are significant only in the higher track (p93, Figure $10, p$-value $=0.036$, see also Appendix, Table 26). Parents' high cultural participation reaches the level of significance in the lower and intermediate as well as in the higher track, but not in schools without tracking (p92). Accordingly, the indirect effects of parental education on students' average grades show a similar pattern (compare Figure 12 and Appendix, Table 28). Parental education has small, significant indirect effects through parents' high cultural participation in the lower and intermediate as well as in the higher track. The indirect effect through students' high cultural participation is significant only in the higher track. However, it is quite small (beta $=0.014)$. 
From a theoretical perspective, social distinction affects grades in each type of school. Distinction impacts grades because social status signals influence the teacher's perception regarding the students' skills and knowledge (compare chapters 2.4.1). A student showing cultural characteristics that indicate higher-class position is seen as more skilled and educated by the teacher as a student who does not exhibit high status signals. Thus, distinction is a more general mechanism that does not substantially vary across the school tracks like school track-specific requirements of skills and knowledge. Therefore, hypotheses about the distinctive function of students' and parents' high cultural participation (H3.C, H6.C) are not clearly supported.

Results with regard to reading are not clear either. Whereas the positive relation of students' reading activities with their average grade is significant in schools without tracking and the higher track, it is not in lower and intermediate tracks (p96, Figure 10). Thus, the indirect effects of parental education on grades through reading activities are significant in schools without tracking and the higher track. The betas vary between 0.019 and 0.041 in schools without tracking and 0.011 and 0.024 in the higher track. Proponents of the common theory argue that reading has a positive effect on grades as reading promotes skills and knowledge crucial in school (H7.C). Just as social distinction, this is a general mechanism that should pertain in each type of school. Hence, results do not confirm this hypothesis.

To evaluate if differential effects across types of school exist with regard to the mediator variables, a regression analysis of students' average grade is conducted including interaction terms of students' and parents' high cultural activities and reading activities with the type of school. Compared to the lower and intermediate track, reading activities are significantly more important for grading in schools without tracking. The standardized regression coefficient of the respective interaction term is 0.094 ( $p=0.030$, compare Appendix, Table 29). 

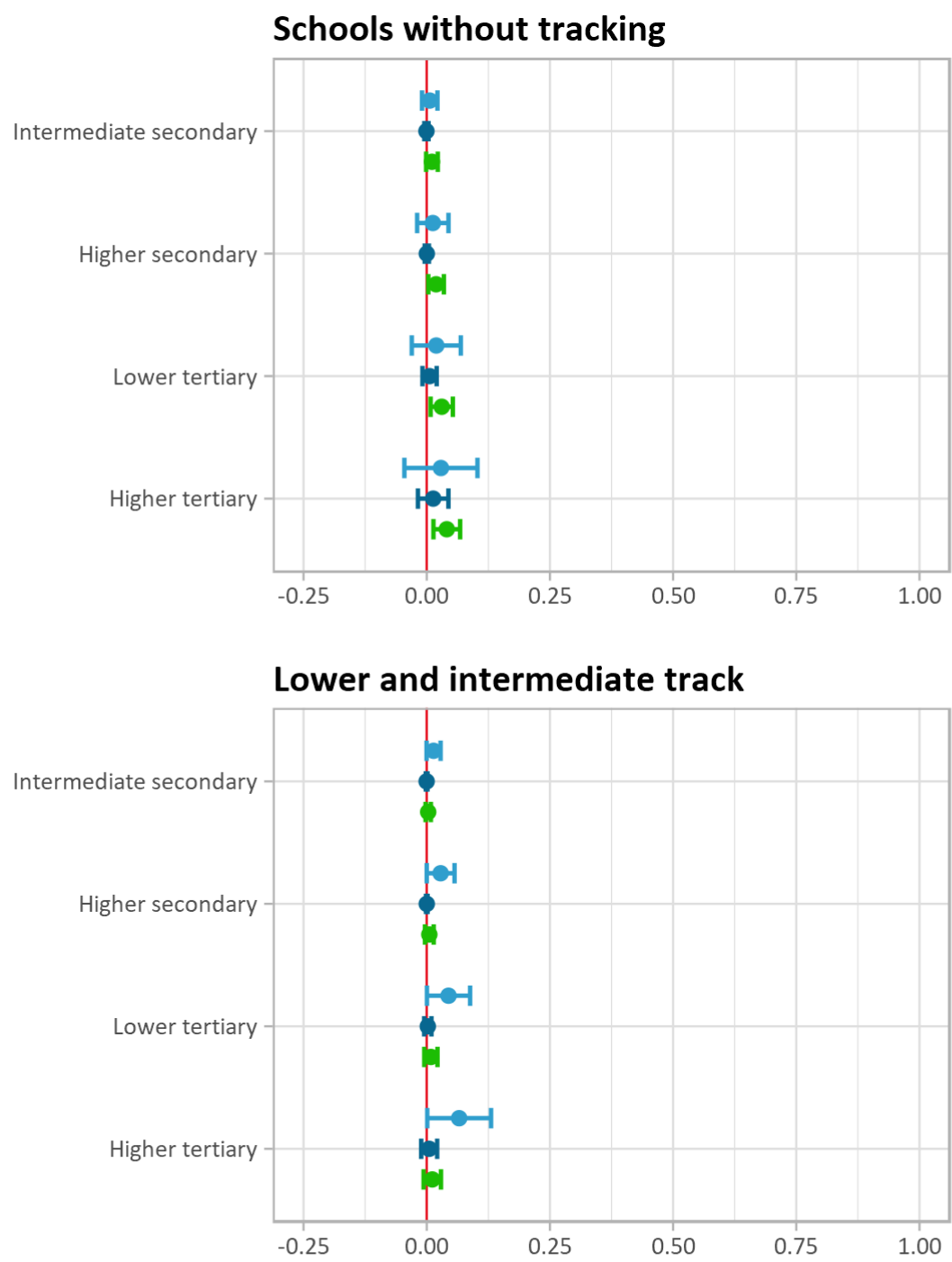

Path

Parents' high cultural participation Students' high cultural participation Reading activities

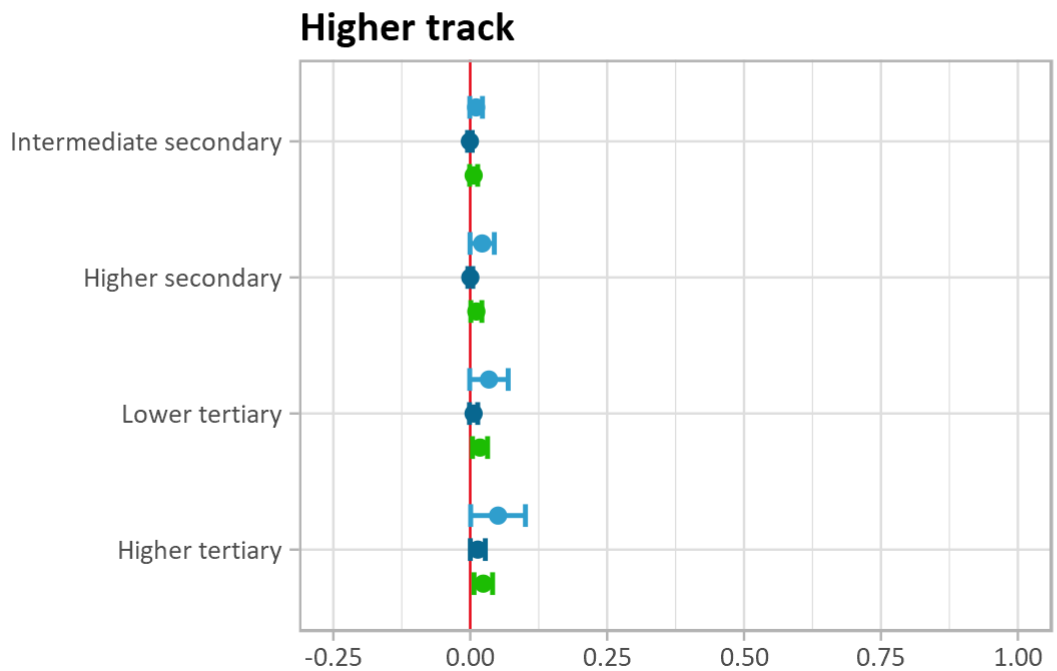

Figure 12: Indirect effects of parental education on students' grades - common model Source: own calculations based on NEPS SC3 7.0.0

Note: Wald confidence intervals are calculated for $\alpha=0.05$. 
In short, cultural capital in terms of the common theory has small or no effects on the average grade of fifth graders depending on the school track (see also Table 11). As the mechanisms put forward by the common theory are independent of school track, findings cast doubt on the theory. It seems unsuitable to explain the social differences in academic achievement.

Table 11: Overview of the results on the impact of cultural capital on academic achievement - common theory

\begin{tabular}{|c|c|c|c|}
\hline Number & Path & Hypothesis & Result \\
\hline H3.C & p93 & $\begin{array}{l}\text { The more students participate in high culture, the } \\
\text { more positive they are assessed by teachers. }\end{array}$ & $x$ \\
\hline H6.C & p92 & $\begin{array}{l}\text { The more parents participate in high culture, the } \\
\text { more positive their children are assessed by teach- } \\
\text { ers. }\end{array}$ & $x$ \\
\hline H7.C & p96 & $\begin{array}{l}\text { The more students read, the better they are as- } \\
\text { sessed by teachers. }\end{array}$ & $x$ \\
\hline
\end{tabular}

Notes: $\checkmark$ indicates that the hypothesis is confirmed, $\mathrm{x}$ indicates that it is falsified.

The revised model

In the next section, the results of the path analysis for the revised model are reported. First, the results on the acquisition of habitus and cultural capital are described, followed by the results on the impact of habitus and cultural capital on academic achievement.

The acquisition of habitus and cultural capital

Path p21 (Figure 13, see also Table 26) shows that higher educated parents conduct high cultural practices more frequently than lower educated parents do. As positive attitudes towards high culture affect high cultural practices, it can be concluded that higher educated parents have more positive attitudes towards high culture (H13.R).

Moreover, parents pass these attitudes on to their children (p32, Figure 13, H11.R). Alternatively, it can be assumed that the effect is shown by the joint participation of parents with their children. Strictly speaking, these two alternative explanations cannot be disentangled with the current data. Shared high cultural practices are a kind of high culture related parenting practices that influence children's attitudes towards high culture 
(H12.R). Therefore, both explanations imply that the measure students' high cultural practices is related to attitudes towards high culture. Furthermore, the effect of parental education on students' high cultural practices is mediated by parents' high cultural practices. The direct effects of parental education on students' high cultural practices are small and negative, but do not significantly differ from zero (p31, Figure 13). Yet, there are significant total effects of lower and higher tertiary education (Figure 14, see also Appendix Table 30). Furthermore, the indirect effects of parental education on students' high cultural practices via parents' high cultural practices are significant, too (Figure 14). Hence, the association of parental education and students' high cultural practices is partially explained by parents' high cultural practices.

The number of books in the home is affected by parental education (p41, Figure 13). Path coefficients are highly significant $(p<0.001)$ and values range from 0.441 (intermediate secondary education) to 1.505 (higher tertiary education). Hence, it can be concluded that higher educated parents have more positive attitudes towards reading (H30.R) and higher passive and active linguistic skills (H22.R and H23.R). However, number of books in the home can only be considered a proxy for these constructs (compare chapter 3.2).

Furthermore, the number of books in the home influences students' attitudes towards reading (p54, Figure 13). Thus, parents' attitudes towards reading seem to affect their children's attitudes (H27.R). The indirect effect of parental education on students' attitudes towards reading through the number of books in the home are significant. Hence, part of the effect of social class on students' attitudes towards reading is mediated by parents' attitudes towards reading (see Figure 14).

In turn, students' attitudes towards reading predict reading practices. Positive attitudes towards reading work as an intrinsic motivation to read. The more students like to read the more they actually do read (H26.R). Judging from the standardized regression coefficient, the effect is quite high ( $p 65$, Figure 13; beta $=0.519$ ). Again, the direct effect of 
parental education on reading practices is mediated by the number of books and students' attitudes towards reading (Figure 14).

Students differ in their passive and active linguistic skills according to parental education (see Figure 14). Differences in reading comprehension and orthographic competence are partially mediated by the number of books in the home (p74 and p84, Figure 13) and reading practices (p76 and p86, Figure 13). These results might be interpreted in a way such that parents' linguistic skills vary across social classes and form the linguistic skills of their children in the context of familial socialization (H17.R and H18.R). Moreover, part of the social inequalities in linguistic skills appears to be explained by class-specific reading practices (H24.R and H25.R). The indirect effects of parental education on reading comprehension and orthographic competence are significant (compare Table 28), but quite small. One interpretation of this finding is that the linguistic skills of parents are less important to explain the social inequalities in linguistic skills of students. Another reading is that the number of books in the home is a mediocre proxy for parental linguistic skills. Taking empirical findings about the acquisition of linguistic skills into account (see chapter 2.3.1), the latter cause is much more likely.

In short, results suggest that parents' and students' attitudes towards high culture differ by social class and these attitudes are transmitted within families. Moreover, students whose parents are more highly educated have higher passive and active linguistic skills than students whose parents are less educated. This is partly due to the higher linguistic skills of the more highly educated parents who pass them on to their children, and partly to differences in reading practices. Parents with higher education give their children more positive attitudes towards reading than lower educated parents do. As a result, these children read more and thus develop their linguistic skills (see also Table 12). 
Table 12: Overview of the results on the acquisition of habitus and cultural capital - revised theory

\begin{tabular}{|c|c|c|c|}
\hline Number & Path & Hypothesis & Result \\
\hline H13.R & $\mathrm{p} 21$ & $\begin{array}{l}\text { Parents from upper social classes have more posi- } \\
\text { tive attitudes towards high culture than parents } \\
\text { from lower social classes have. }\end{array}$ & $\checkmark$ \\
\hline H11.R & p32 & $\begin{array}{l}\text { The more positive the parents' attitudes towards } \\
\text { high culture, the more positive are the students' } \\
\text { attitudes towards high culture. }\end{array}$ & $\checkmark$ \\
\hline H30.R & $\mathrm{p} 41$ & $\begin{array}{l}\text { The higher the social class of the parents, the more } \\
\text { positive are their attitudes towards reading. }\end{array}$ & $\checkmark$ \\
\hline $\mathrm{H} 22 . \mathrm{R}$ & $\mathrm{p} 41$ & $\begin{array}{l}\text { The higher the social class of the parents, the } \\
\text { higher are their passive linguistic skills. }\end{array}$ & $\checkmark$ \\
\hline$H 23 . R$ & $\mathrm{p} 41$ & $\begin{array}{l}\text { The higher the social class of the parents, the } \\
\text { higher are their active linguistic skills. }\end{array}$ & $\checkmark$ \\
\hline $\mathrm{H} 27 . \mathrm{R}$ & p54 & $\begin{array}{l}\text { The more positive parents' attitudes towards read- } \\
\text { ing, the more positive are the student's attitudes } \\
\text { towards reading. }\end{array}$ & $\checkmark$ \\
\hline H26.R & p65 & $\begin{array}{l}\text { The more positive students' attitudes towards } \\
\text { reading, the more they read. }\end{array}$ & $\checkmark$ \\
\hline H17.R & p74 & $\begin{array}{l}\text { The higher the passive linguistic skills of the par- } \\
\text { ents, the higher are the student's passive linguistic } \\
\text { skills. }\end{array}$ & $\checkmark$ \\
\hline H18.R & p84 & $\begin{array}{l}\text { The higher the active linguistic skills of the parents, } \\
\text { the higher are the student's active linguistic skills. }\end{array}$ & $\checkmark$ \\
\hline $\mathrm{H} 24 . \mathrm{R}$ & p76 & $\begin{array}{l}\text { The more students read, the higher their passive } \\
\text { linguistic skills. }\end{array}$ & $\checkmark$ \\
\hline $\mathrm{H} 25 . \mathrm{R}$ & p86 & $\begin{array}{l}\text { The more students read, the higher their active lin- } \\
\text { guistic skills. }\end{array}$ & $\checkmark$ \\
\hline
\end{tabular}

Notes: $\checkmark$ indicates that the hypothesis is confirmed, $\mathrm{x}$ indicates that it is falsified. 

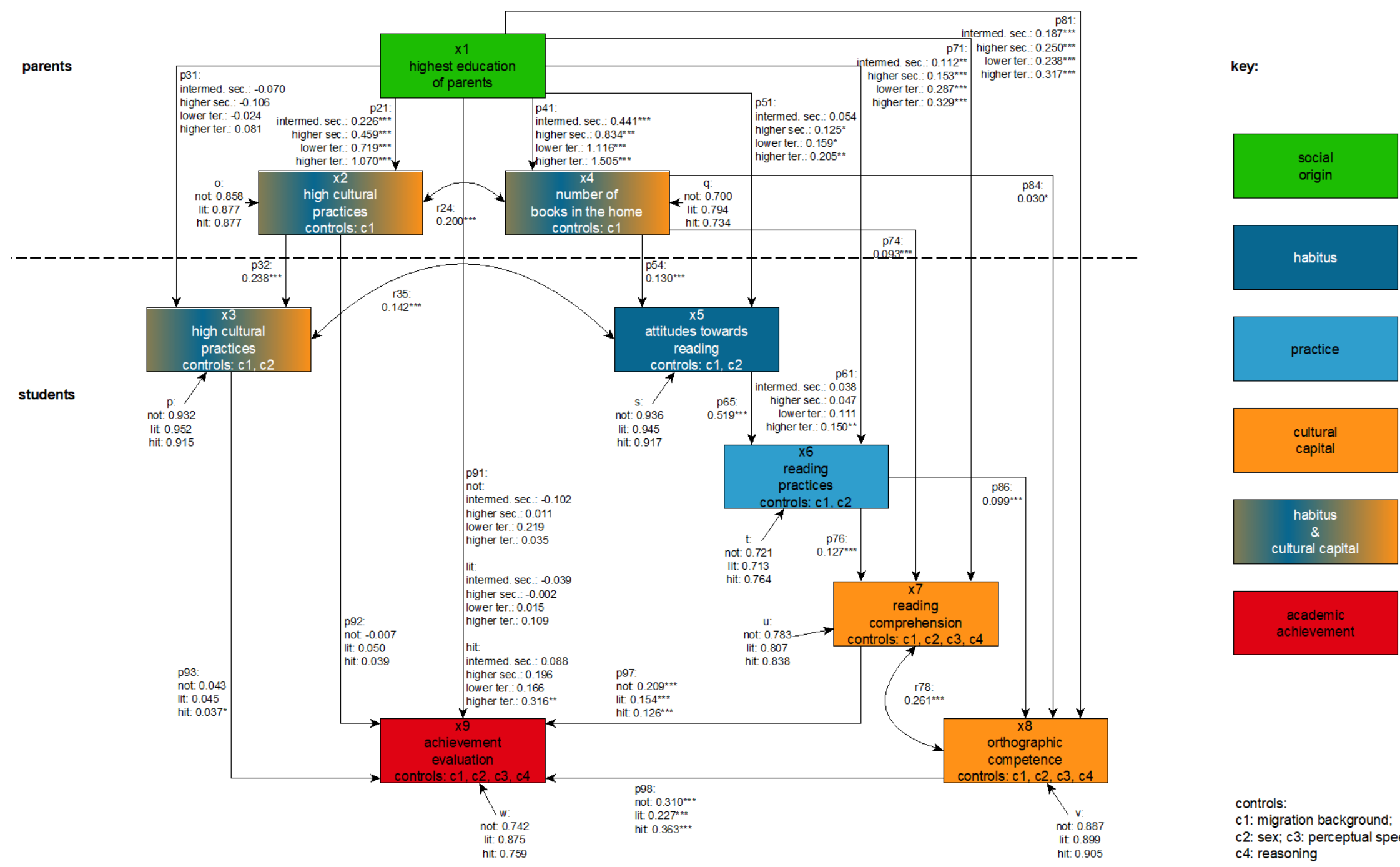

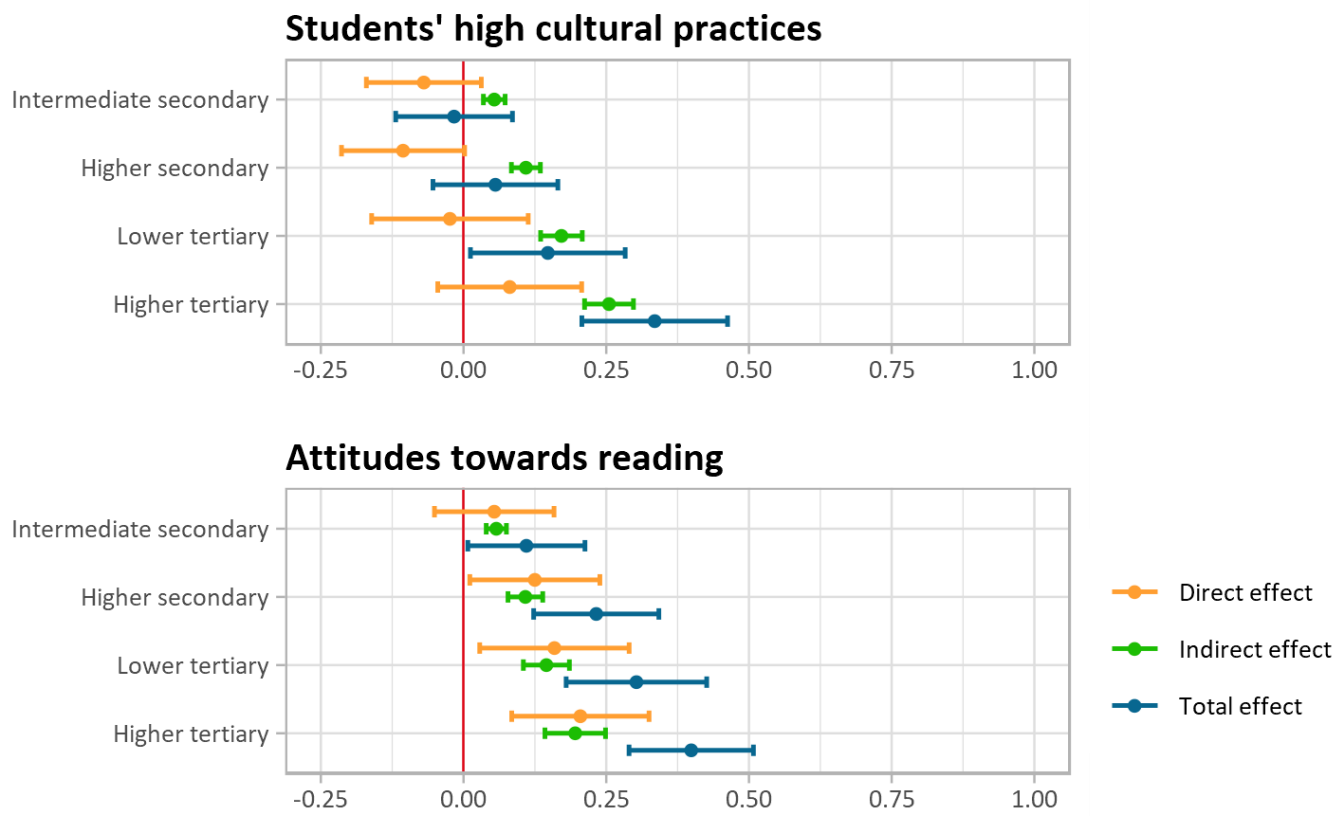

Reading practices

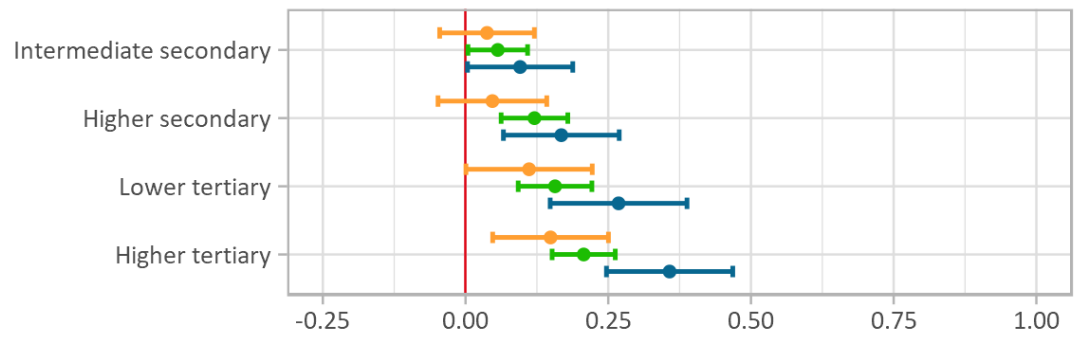

Reading comprehension

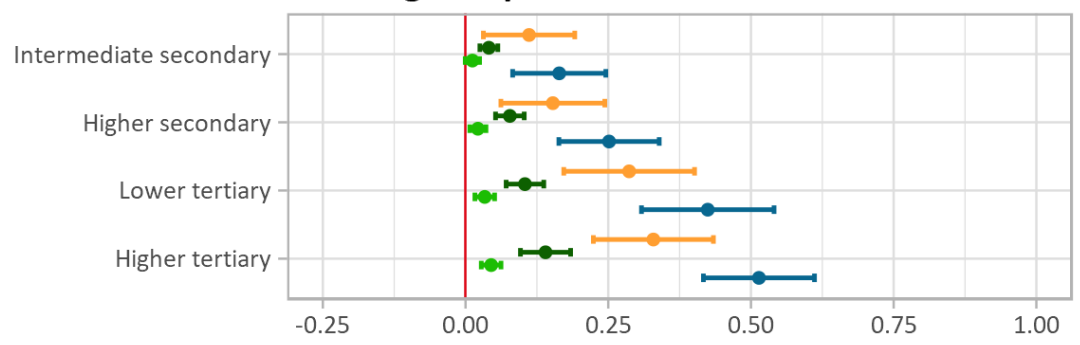

- Direct effect Indirect effect via number of books in the home Indirect effect

Orthographic competence

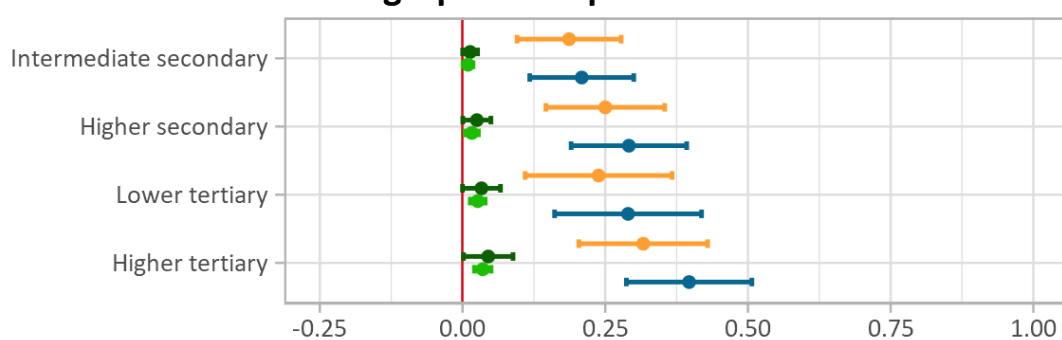

$\rightarrow$ via reading practices

$\multimap$ Total effect

Figure 14: Direct, indirect, and total effects of parental education on mediator variables - revised model Source: own calculations based on NEPS SC3 7.0.0

Note: Wald confidence intervals are calculated for $\alpha=0.05$. 
The impact of habitus and cultural capital on academic achievement

According to the results, students' high cultural practices have a small significant effect on the average grade in the higher track (H3.R; hit: p93; beta $=0.037)$. Path coefficients for schools without tracking and for the lower and intermediate tracks are comparable in size but non-significant (not: p93, lit: p93). Additionally, there are no significant indirect effects of parental education on students' grades through students' high cultural practices (compare Figure 15, see also Appendix Table 31). Hence, the argument that students' attitudes towards high culture influence teachers' assessments (H3.R) cannot be confirmed. In the common model, there is a small indirect effect in the higher track. However, when linguistic skills are considered as in the revised model this indirect effect turns insignificant. This suggests that part of the effect of high cultural indicators is caused by some variance shared with linguistic skills.

Moreover, the results show that there is no effect of high cultural practices of parents on school grades (p92). Theory holds that individuals showing positive attitudes towards high culture are viewed as belonging to the educated classes. Further, it is argued that teachers evaluate the performance of students from the seemingly educated classes higher than of those students that lack those signals (H4.R). Results do not support that parents' attitudes towards high culture are a means for social distinction.

However, passive and active linguistic skills have positive and significant effects on students' average grade (H15.R, H16.R). This result is consistent across the different types of school. More specifically, the standardized regression coefficient of reading comprehension (p97) in schools without tracking is 0.209 , in the lower and intermediate track 0.154 , and in the higher track 0.126 . The corresponding $p$-values are 0.000 . Orthographic competence has a higher impact on grades in the fifth grade (p98). Betas are 0.310 in schools without tracking, 0.227 in the lower and intermediate track, and 0.363 in the higher track. Again, p-values are 0.000 . The indirect effects of parental education on students' grades via reading comprehension and orthographic competence are positive 
and consistently significant irrespectively of the type of school (Figure 15). The higher parents are educated, the higher students' linguistic skills, and the better their grades.

According to regression analysis, significant differences in the effects of the mediator variables exist across the different types of school. Students' grades are regressed on the mediator variables of the revised model ${ }^{36}$ including interaction terms with type of school. Compared to the lower and intermediate track, there is a significant, positive interaction effect of orthographic competence and the higher track dummy on students' average grade (compare Appendix, Table 32). Hence, orthographic competence is especially influential in the higher track.

In sum, the social inequalities in academic achievement are caused by unequal linguistic skills rather than by distinctive attitudes towards high culture.

Table 13: Overview of the results on the impact of habitus and cultural capital on academic achievement - revised theory

\begin{tabular}{|c|c|c|c|}
\hline Number & Path & Hypothesis & Result \\
\hline H3.R & p93 & $\begin{array}{l}\text { The more positive students' attitudes towards high } \\
\text { culture are, the more positive teachers assess the } \\
\text { students. }\end{array}$ & $x$ \\
\hline H4.R & p92 & $\begin{array}{l}\text { The more positive parents' attitudes towards high } \\
\text { culture are, the more positive teachers assess the } \\
\text { students. }\end{array}$ & $x$ \\
\hline H15.R & p97 & $\begin{array}{l}\text { The higher students' passive linguistic skills, the } \\
\text { better they are assessed by teachers. }\end{array}$ & $\checkmark$ \\
\hline H16.R & p98 & $\begin{array}{l}\text { The higher students' active linguistic skills are, the } \\
\text { better they are assessed by teachers. }\end{array}$ & $\checkmark$ \\
\hline
\end{tabular}

Notes: $\checkmark$ indicates that the hypothesis is confirmed, $\mathrm{x}$ indicates that it is falsified.

${ }^{36}$ Students' and parents' high cultural practices, reading comprehension, orthographic competence 

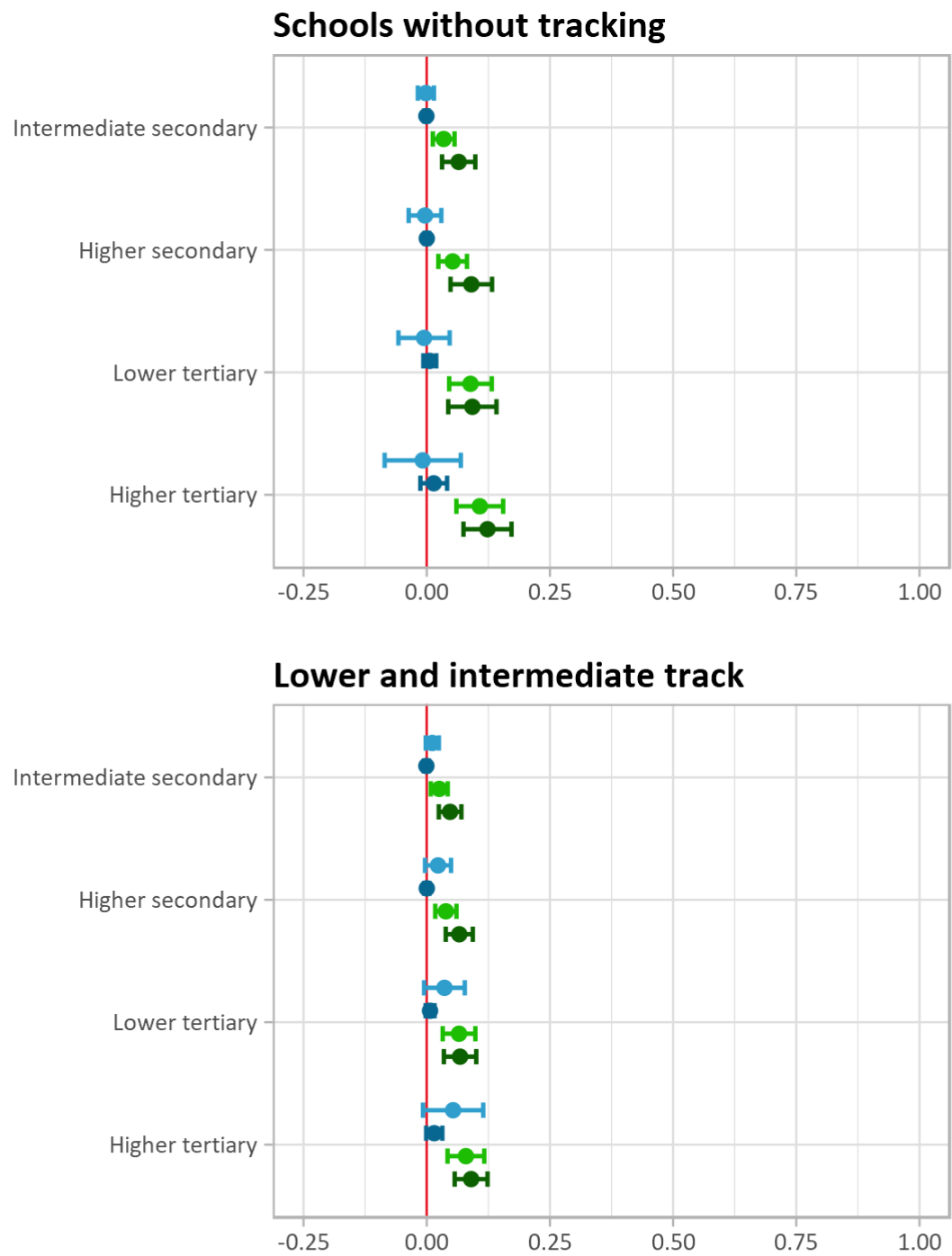

Path

Parents' high cultural participation Students' high cultural participation Reading

- comprehension

- Orthographic competence

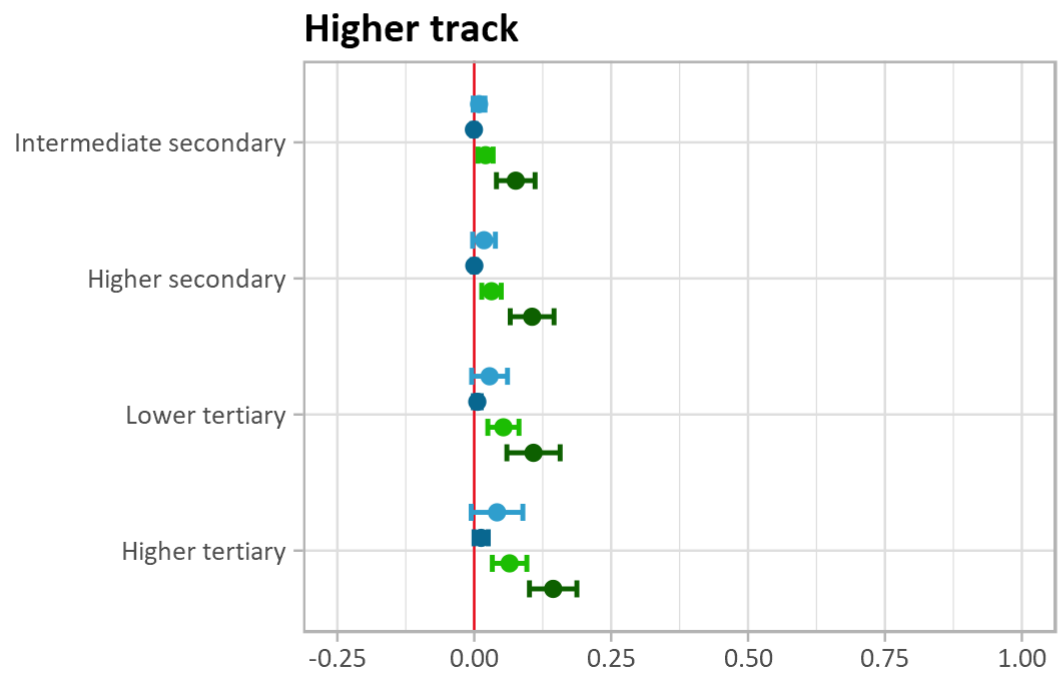

Figure 15: Indirect effects of parental education on students' grades - revised model Source: own calculations based on NEPS SC3 7.0.0

Note: Wald confidence intervals are calculated for $\alpha=0.05$. 
Comparison of the common and the revised model

As described in chapter 3.4, the explanatory power of the common and the revised model regarding social inequalities in grades is assessed by comparing the respective standardized indirect effects of parental education on the average grade. Besides, the total and the direct effects are also shown for the sake of completeness (Figure 16, see also Appendix Table 28, Table 31).

As expected, the total effects of the common and the revised model are more or less the same within the various types of school. The point estimates with regard to schools without tracking and the higher track differ between the models. However, the confidence intervals overlap for the most part. Hence, it cannot be concluded that the total effects differ significantly. The total effects of parental education on students' grades in the lower and intermediate track are almost identical. Moreover, it can be assumed that the total effects of the models increase by the level of parental education. However, this assumption is not met with regard to schools without tracking. The total effect of lower tertiary education on students' grades is higher than the total effect of higher tertiary education. This is true for the common and the revised model. The comparability of the models regarding the total effects of lower and higher tertiary education suggests that they are valid in the revised model despite the sub-optimal sub-sample size to parameters ratio N:q. (compare chapter 3.5.5). Possibly, this results from the low number of cases with these characteristics. Regarding the non-imputed data, 57 students attend a school without tracking whose parents hold a lower tertiary degree (see Appendix, Table 33).

Considering the direct effects, it can be assumed that the direct effects decrease when the indirect effects increase. In fact, the direct effects of the revised model are smaller and the indirect effects are bigger than those of the common model are (Figure 16). With regard to the association of parental education and effect size, the same pattern occurs as for the total effects. The higher parental education is, the higher the direct effect except for lower tertiary education in schools without tracking. Moreover, in the 
higher track the point estimate of the direct effect of higher secondary education is slightly above the one of lower tertiary education.

However, the crucial measures in terms of mediation are the indirect effects. The comparison of the total indirect effects in the common and the revised model shows a clear pattern. First, indirect effects increase steadily by parental education in both models. Secondly, the total indirect effects of the revised model are consistently higher than those of the common model are. In schools without tracking, the standardized total indirect effects in the common model range from 0.016 to 0.083 and in the revised model from 0.097 to 0.237 (compare Appendix, Table 28 and Table 31). In the lower and intermediate track, they range from 0.016 to 0.082 in the common model and 0.084 and 0.239 in the revised model. In the higher track, the standardized total indirect effects vary between 0.016 and 0.089 in the common model and between 0.105 and 0.263 in the revised model. A comparison of the confidence intervals of the revised and the common model suggest that most of these differences are significant. Only two of the confidence intervals partially overlap, those of lower and higher tertiary education of students that attend schools without tracking.

Overall, the effect of parental education on students' grades is mediated by the common and revised model to some extent. However, the total indirect effects are considerably higher in the revised model than in the common model.

Next, the squared semi-partial correlations of the concurrent models are compared, that is, the relative share of the variance of students' grades that is explained by parental education and not by the mediator variables in the model (compare chapter 3.4). As a reference, also the R-square of students' average grade regressed on parental education only is given (Figure 17). With regard to schools without tracking, the squared semi-partial correlation of students' grades and parental education in the common model equals 0.03 , whereas in the revised model it equals 0.008 . The R-square is 0.06 . Looking at the lower and intermediate track, the squared semi-partial correlation is 0.006 in the common model versus 0.001 in the revised model. The R-square equals 0.011 . Considering 

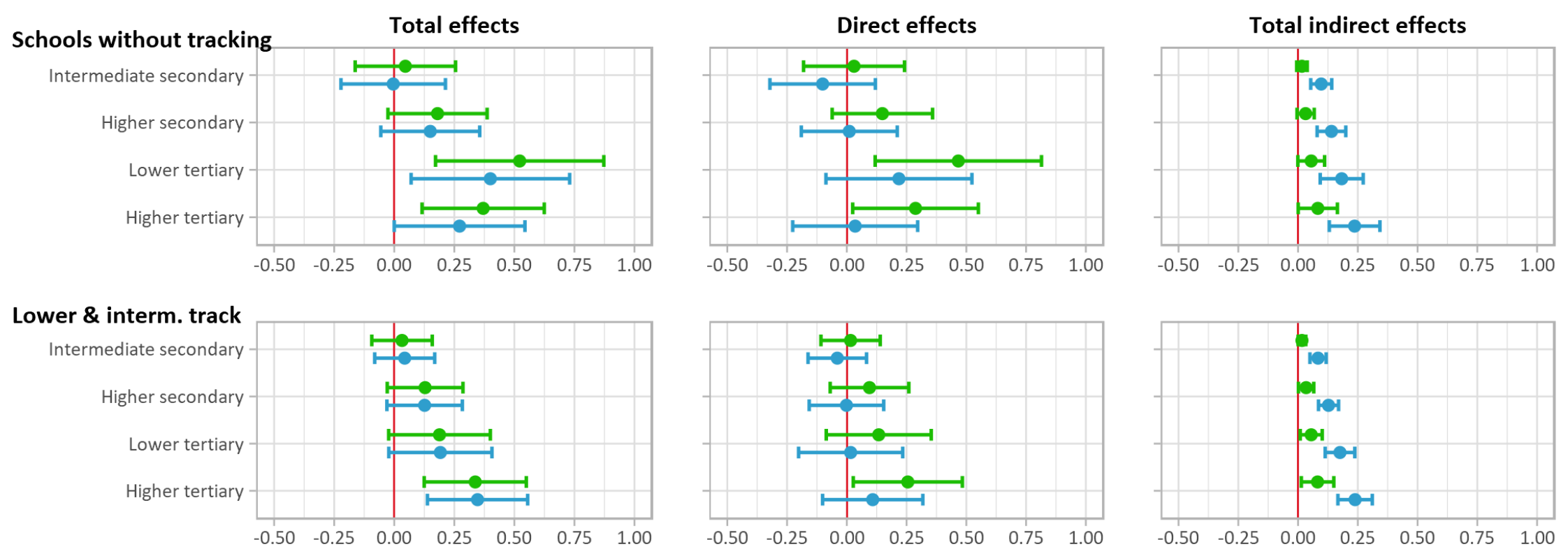

\section{Higher track}

Intermediate secondary -

Higher secondary

Lower tertiary

Higher tertiary -
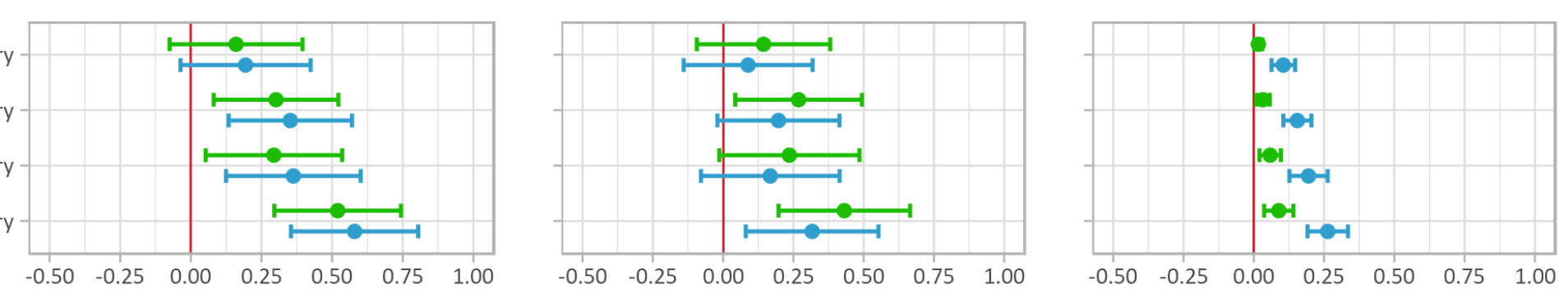

$\rightarrow$ Common model $\rightarrow$ Revised model

Figure 16: Comparison of the total, direct, and total indirect effects of parental education on students' grades

Source: own calculations based on NEPS SC3 7.0.0

Notes: Effects after controlling for migration background, sex, perceptual speed, reasoning. Wald confidence intervals are calculated for $\alpha=0.05$. 
the higher track, the squared semi-partial correlation is 0.017 in the common model and 0.009 in the revised model. The respective R-square amounts to 0.033 . The differences in the R-square between students' average grade and parental education across the types of school suggest that the impact of parental education is highest in schools without tracking and lowest in the lower and intermediate track. The different explanatorypower of social origin regarding grades in the different types of schools can be explained by the differential effects of the mediator variables on students' average grades between types of school (p92, p93, p96 Figure 10 and p92, p93, p97, p98 Figure 13).

In sum, the squared semi-partial correlations of students' grades and parental education are consistently lower in the revised model than in the common model. Therefore, the revised model is more suitable to explain social inequalities in academic achievement than the common model. 

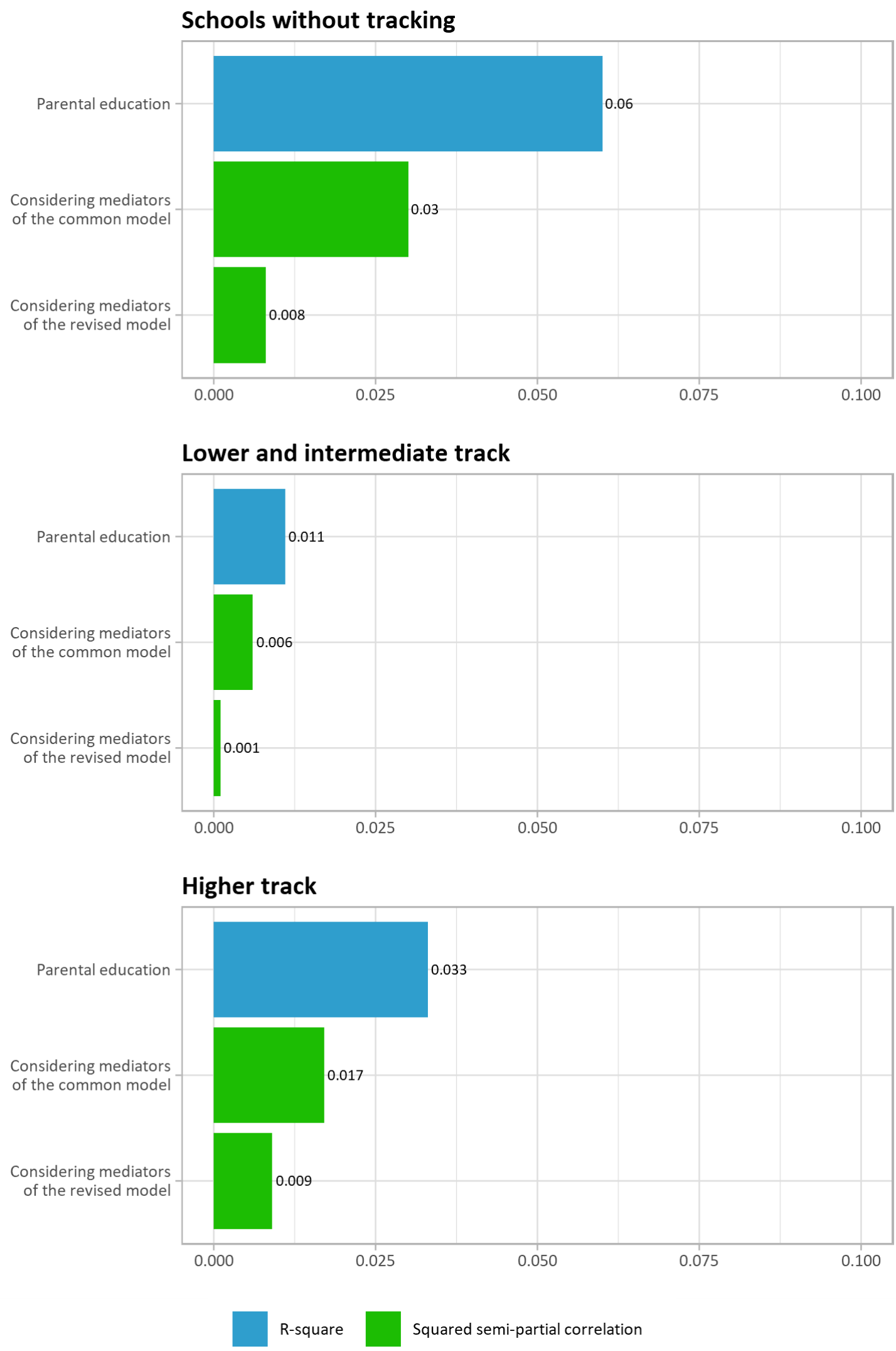

Figure 17: Comparison of the semi-partial correlations between students' average grades and parental education Source: own calculations based on NEPS SC3 7.0.0 


\section{Conclusion}

The following chapter contains a summary of the thesis and a discussion of the most important results. It also addresses implications for intervention strategies. The thesis ends with a description of limitations of the presented research work and a focus on aspects that are suitable for further research.

\subsection{Summary}

Cultural capital theory, which goes back to the concept of Bourdieu (1977a), is an oftenused approach to explain social inequalities in educational success and academic achievement. However, Bourdieu's approach is seriously criticized: unclear definitions and hypotheses, and insufficient empirical analyses. Also the common interpretation of the theory by DiMaggio (1982) is criticized for a low explanatory power, an overly narrow interpretation of the concept, and the negligence of the related concept of habitus. In this thesis, these critiques of the theory are taken up and the existing readings of the cultural capital theory and related interdisciplinary research are interpreted, explicated, and restructured in order to obtain a revised version of the theory. Hence, in a first step a clear, testable theory of cultural capital and habitus is put forward that has substantial explanatory power with regard to social inequalities in academic achievement. Furthermore, the revised theory has to meet the requirements to inform about starting points for effective interventions to reduce the inequalities. Since the common theory forms the frame of reference for the revised theory, the common theory is presented first.

\section{The common theory}

The common theory defines cultural capital as positive attitudes towards, participation in, and knowledge about high culture.

The common cultural capital theory is a theory of class conflict that focusses on high cultural aspects ignoring linguistic skills. It is argued that high culture is characteristic for the upper classes and only for the upper classes. Participation in or familiarity with high culture constitute cultural capital that signals membership of the upper-classes (DiMaggio, 1982, pp. 189-190). This cultural capital is transmitted within the family of origin. 
Moreover, the educational system is dominated by the upper social classes possessing the most cultural capital. They secure their dominant position in the social hierarchy by rewarding cultural capital in the educational system (e.g. Bourdieu, 1977a). Many studies based on this interpretation of cultural capital refer to DiMaggio's well-known study from 1982 (Goldthorpe, 2007; Kingston, 2001; Lareau \& Weininger, 2003). Another dimension of cultural capital usually complements this view of cultural capital: reading. Reading activities are socialized within the family of origin and they promote skills relevant for educational success. Still, these skills are not explicitly part of the theoretical model how it is typically tested.

More precisely, the common theory assumes that parents' and students' high cultural knowledge, attitudes towards, and participation in high culture affect academic achievement because of their distinctive character. Furthermore, reading positively affects academic achievement by raising relevant skills, for example linguistic skills. The dimensions of cultural capital are transmitted within the family of origin.

Path analysis confirms that higher-class parents participate more often in high culture and that high cultural participation is transmitted within the family. Results concerning the effect of parents' and students' high cultural participation on academic achievement are inconsistent between the different types of school. They are significant only in some types of school. The underlying mechanism of social distinction, however, is assumed to be effective across the school system and should operate in any type of school. The credibility of the respective hypotheses is therefore doubtful. Correspondingly, the standardized indirect effects of social class on academic achievement through parents' and student' high cultural participation are quite small and only partially significant.

Results regarding reading activities show a very similar pattern. Just as high cultural participation, reading activities are assumed to affect academic achievement independent from the type of school attended. Hence, the corresponding hypotheses cannot be confirmed either. 
The revised theory

The first step of this thesis is to formulate a useful theory as defined by Opp. The most fundamental question to be answered to revise the theory is:

(1) How can the central terms of a useful theory of cultural capital and habitus be defined?

Based on a systematic summary of a variety of differing definitions of cultural capital and habitus, clear, consistent, disjoint, and complementary definitions are derived (chapter 2.1.3). Cultural capital is defined as cognitive and linguistic skills and knowledge. Habitus is specified as internalized characteristics that guide action and behavior, that is attitudes, norms and values, idealistic aspirations and beliefs about one's own skills and knowledge. Action and behavior that is determined by habitus is called practice.

In the next step, the relations of social origin, dimensions of cultural capital and habitus, and academic achievement are clarified (chapter 2.4.2). Based on relevant research findings of sociology, psychology, and educational research and a description of the presumed causal mechanisms, hypotheses are formulated. The central questions guiding this process are:

(2) How are cultural capital and habitus acquired?

(3) How does cultural capital and habitus affect academic achievement?

Briefly, the revised theory of cultural capital and habitus explains social inequalities in academic achievement by skills, knowledge, and motivational determinants. These factors are unequally distributed across social classes and they are mainly acquired by socialization in the family of origin. They either have a distinctive effect, promote the students' learning capacity, or are explicit criteria of achievement assessments. Thus, the revised theory claims that students from the upper classes perform better in school than 
lower-class students as upper-class parents provide more favorable socialization contexts regarding skills, knowledge, and motivational factors crucial for students' assessment by teachers. Social distinction plays a subordinate role. Hence, it is less of a class conflict theory but more a theory of unequal socialization.

More specifically, it is assumed that inequalities are primarily caused by differences in passive and active linguistic skills between social classes. Passive linguistic skills determine the ability to learn and to comprehend school lessons. Moreover, sometimes they are themselves the subject of evaluations. Active linguistic skills are evaluation criteria, too. Passive and active linguistic skills are acquired directly or indirectly by socialization. Parents shape children's linguistic skills directly as their language use serves as language model and language-related parenting practices give children access to communicative opportunities (Hoff, 2006). Furthermore, parents influence children's linguistic skills indirectly as they affect children's attitudes towards reading, and hence, their reading practice.

Additionally, the revised theory holds that social distinction by class differences in attitudes towards high culture and high cultural knowledge influence academic achievement. Teachers perceive students with positive attitudes towards high culture and high cultural knowledge as more educated and talented than students without those characteristics. Hence, they assess the former more positive than the latter. Moreover, parents' attitudes and knowledge are assumed to affect teachers' assessment. Parents with positive attitudes and substantial knowledge are also assessed as being educated. Therefore, their children seem to have good educational prospects. This might also affect teachers' assessments. Parents transmit attitudes towards high culture and high cultural knowledge to their children by everyday interaction and high culture-related parenting practices. Similar to linguistic skills, parents also influence high cultural knowledge indirectly. Positive attitudes towards high culture motivate children to conduct high cultural practices. In turn, high cultural practices form learning opportunities that enable children to acquire high cultural knowledge. 
The second step of the thesis is to test the revised theory. Empirical results support that passive and active linguistic skills are unequally distributed across social classes and have a substantial positive effect on academic achievement in the fifth grade. Further analyses suggest that effect size differs between types of school. More specifically, the influence of active linguistic skills measured by orthographic competence on the average grade is significantly higher in the higher track (Gymnasium) than in the lower and intermediate track and schools without tracking. Moreover, analyses indicate that passive and active linguistic skills are affected by parents' linguistic skills. In addition, results confirm the indirect effect of parental characteristics on passive and active linguistic skills via parents' attitudes towards reading, students' attitudes towards reading, and reading practice.

Moreover, analyses imply that higher-class parents have more positive attitudes towards high culture than lower-class parents have and that these attitudes are transmitted to children within the family of origin. However, the results rather speak against an effect of social distinction through attitudes towards high culture.

Since the theory claims a mediator function of cultural capital and habitus, in addition to testing the individual hypotheses, it is tested whether significant indirect effects can be proven for the postulated chains of effects.

(4) Do the postulated causal pathways make a significant contribution to the explanation of social inequalities in academic achievement?

The indirect effects of social class on academic achievement via active and passive linguistic skills are significant and substantial. However, there are no significant indirect effects of social class on academic achievement through parents' or students' attitudes towards high culture.

Comparison of the common and the revised theory

In order to substantiate the reasonableness of the revised theory, it must prove empirically better in direct comparison with the common theory. The most crucial criterion for 
the performance of the theories is their explanatory power with regard to social inequalities in academic achievement. Hence, the third step of the thesis is to compare this explanatory power. This is depicted by the question:

(5) Does the revised theory show a higher explanatory power with regard to social inequalities in academic achievement than the common theory?

To answer this question, two strategies are used. The total standardized indirect effects of parental education on students' grades are compared. Furthermore, the squared semi-partial correlations between parental education and students' grades are contrasted when mediator variables (cultural capital, habitus) are controlled.

The results with regard to the total standardized indirect effects follow a clear and consistent pattern. As expected, the indirect effects increase by the level of parental education in both models. However, the total standardized indirect effects of the revised model are substantially larger than those of the common model are. Moreover, when the respective mediator variables are controlled, the squared semi-partial correlations of the revised model are considerably lower than those of the common model are. Overall, these results clearly indicate that the revised model has a higher explanatory power with regard to social inequalities in academic achievement than the common model.

\subsection{Discussion}

In total, the findings suggest the following conclusions. The common cultural capital theory as it is described in this thesis, only has very limited explanatory power regarding social inequalities in academic achievement in the fifth grade in Germany. This is consistent with Kingston's critique and might be explained by the narrow interpretation of cultural capital in terms of high culture (Kingston, 2001). The common extension by reading does not sufficiently increase the explanatory value of the theory, as this extension remains too superficial and does not take into account the underlying mechanisms of the reading effect, that is, linguistic skills. 
The revised theory performs considerably better than the common theory as linguistic skills are taken into account. Passive and active linguistic skills explain not only differences in grades in general, but also differences in grades between students from different social origins. Thus, linguistic skills are important mediators regarding the effect of social origin on academic achievement. This clearly supports the use of the revised model instead of the common model. Generally, in the fifth grade, active linguistic skills seem to be a little more important than passive skills. Additionally, findings suggest that there are differential effects of linguistic skills on academic achievement across the types of school. Accordingly, active language skills are more important in the higher track (Gymnasium) than in the other types of school.

In contrast, social distinction by attitudes towards high culture do not influence students' assessment by teachers in lower secondary school in Germany. There are two alternative explanations for this finding: (1) Social distinction does not affect academic achievement at all. (2) Social distinction does influence academic achievement, but it operates through other characteristics. These characteristics could entail a more qualitative aspect of high culture: high cultural knowledge (Goßmann \& Mätzke, 2019). Alternatively, as status signals are arbitrary, any other characteristic shared by a certain group could serve as a means of social distinction (Bourdieu \& Passeron, 1977, pp. 2324; Weber, 1978, p. 932). Bourdieu and Passeron (1971, pp. 109-115) argue that the students' language use has a distinctive effect. Possibly, the students' choice of words, accent, and intonation affect their assessments by teachers net of linguistic skills (compare chapter 2.4.2). This reasoning is supported, for example, by findings of Gärtig et al. (2010). They show that people who speak standard German are perceived as more educated than people who speak a dialect. Further studies taking high cultural knowledge and distinctive language use into account could help to answer the question if and how social distinction affects academic achievement.

However, findings of this thesis do not support effects of socially distinctive high cultural practices, but effects of the relevant skills and motivational factors socialized in the family of origin. Hence, it seems not to be about class-conflict fought by "exclusionary class- 
related practices and dispositions" (Kingston, 2001, p. 88), but about class-related home learning environments and parenting practices (see also Kingston, 2001, pp. 96-97). From this perspective, explaining social inequalities in academic achievement primarily means to understand what the relevant skills, fields of knowledge, and motivational factors are, how they are acquired in the family of origin, and how the conditions of acquisition differ between families with different social status.

It can be argued that skills and, hence, the effects of skills on academic achievement are based on individual talent and effort (Katsillis \& Rubinson, 1990, p. 278). Therefore, inequalities in academic achievement due to skills can be viewed as legitimate. However, research on the acquisition of skills leaves no doubt that skills are influenced by social origin to a large extent (compare chapter 2.3.1 and chapter 3.5.7). Social influences determine whether the genetic potential with regard to cognitive abilities is exploited (Guo \& Stearns, 2002). Skills, in turn, are major explanatory factors for academic achievement (compare chapter 2.3 .2 and chapter 3.5 .7 ). Thus, social inequalities in academic achievement are driven by social inequalities in skills. If the aim is to reduce social inequalities in academic achievement, skills have to be considered, their acquisition, and measures to reduce social inequalities in its acquisition. The reduction of educational inequalities is not only a question of equal educational opportunities and social participation, but also a question of exploiting the potential for economic productivity and overall social prosperity.

Comparing the results of the common and the revised model reveals that the common model shows small, but significant effects of parents' high cultural participation on academic achievement, but the revised model does not. This can be explained by the fact that the revised model comprises passive and active linguistic skills and that these linguistic skills are correlated with parents' high cultural participation ( $r=0.2591$ and $r=$ 0.1933; see Appendix, Table 34). Assuming this is true, the common model suffers from omitted variables bias. Parents who participate more often in high culture are also more successful in promoting their children's linguistic skills. Sullivan (2001, pp. 907-908) 
shows a similar pattern. The significant relation between the cultural activities of parents and the grade on the school-leaving certificate is much less strong and is no longer significant when students' linguistic skills and cultural knowledge are included in the model.

The common model has a better model fit than revised model (see chapter 3.5.6). Thus, one might conclude that the common theory should be preferred. However, this is a fallacy. The common model has a better model fit because the overall empirical correlations between the variables in the model are predicted more precisely as in the revised model. Essentially, there are two reasons for this. (1) The revised model is more complex and involves more variables. Generally, this tends to make model fit worse (Kline, 2011). More correlation coefficients must be predicted correctly. (2) The data used for the analyses contains a rich set of relevant variables. There are measures explicitly developed to depict the theoretical constructs of the common theory.

\subsection{Practical implications}

The potential for interventions that can be derived from the revised theory is associated with the promotion of linguistic skills of children in lower-class families. There are several ways how this can be achieved. Lower-class parents can be encouraged to develop their children's linguistic skills actively from an early age. Parents from lower social classes have a lower self-efficacy regarding their influence on their children's cognitive development (Brody, Flor, \& Gibson, 1999). Encouraging these parents to develop their children's linguistic skills actively can narrow the social gap of language development. For example, parents can achieve this by talking more to their children and giving more responses to their children that are related to the children's utterances. Even though these parents have on average lower linguistic skills than higher class parents this probably furthers their children's language development as findings (Hoff, 2006) suggest that besides quality of child-directed speech also quantity as well as responsiveness affects children's language development. 
Additionally, measures can be taken to compensate for the lack of the qualitative dimension of language development. The linguistic skills of disadvantaged children can be enhanced by providing them with additional, developmentally appropriate, and highquality language training in an institutional context as early as possible.

Moreover, for disadvantaged students, language levels in the classroom can be adjusted to take account of social differences in language development and their impact on academic achievement. It might be an approach to form learning groups according to linguistic skills within the different school types.

Another way to promote children's linguistic skills is to read to them. Hence, lower-class parents should be encouraged to read more to their children. Studies show that book reading provides language experiences that are particularly advantageous (Hoff, 2006). Furthermore, these experiences do not differ by social status. Additionally, reading aloud to children furthers positive attitudes towards reading (Demir-Lira et al., 2019). This can motivate children to read more and thus to develop their linguistic skills.

Additionally, other persons can read to children from disadvantaged families, for example in day care centers. Honorary reading mentors might support the staff as educators often lack the time in daily routine to read to the children extensively and regularly.

Furthermore, parents should provide a reading affine climate at home. Thus, children are more likely to develop positive attitudes towards reading. Other measures in primary or lower secondary schools to further such attitudes can be implemented as well. Children with positive attitudes towards reading are more prone to read. This furthers their linguistic skills.

However, interventions aiming at social distinction are very unlikely to succeed. If it is true that the upper social classes obtain advantages in the educational system by social distinction and that those become unsuitable due to intervention, it has to be assumed that the upper social classes develop alternative means of social distinction. 


\subsection{Limitations and future research}

The interpretation and restructuring of different readings of a theory into a coherent, useful theory means excluding some definitions that can be found in the literature. This always represents a more or less subjective choice, even with a high scientific standard.

The scientific background of the researcher and the subjective focus influences the selection and formulation of the hypotheses. Due to vague definitions, unclear theoretical constructs, and partially non-explicit interrelations in the scientific discussion about cultural capital, there is a great variety of possible hypotheses. A theory should contain a consistent set of hypotheses suitable to explain the acquisition of cultural capital and habitus and their impact on academic achievement. Hence, choices among the possible hypotheses have to be made. Most of the hypotheses of the revised theory are based on previous findings from sociology, psychology, and educational research. Of course, there are other reasonable hypotheses that could be put forward. However, these hypotheses should be theoretically well grounded and the assumed underlying causal mechanisms should be made explicit. This furthers the comprehensibility of the hypotheses, their testability, and a fruitful scientific discussion. For example, other dimensions of cultural capital could be considered. In this thesis, cultural capital is defined as cognitive and linguistic skills and knowledge. Hence, mathematical skills or general knowledge could also be taken into account. Both represent rather basic skills respectively knowledge that affect academic achievement and they are acquired in the family of origin at least to some extent. These characteristics make them well suited for explaining social inequalities in academic achievement. Additionally, further dimensions of habitus could be considered that influence students' motivation for learning and school-related practices (compare chapter 2.2.2), for example attitudes towards education, idealistic aspirations, and academic self-concept. Students who have positive attitudes towards education, a positive academic self-concept, and high idealistic aspiration are likely to be more diligent and work harder in school than students with negative attitudes and a low aspiration. This exerts a twofold effect on academic achievement (Farkas et al., 
1990): School learning furthers skills and knowledge relevant for achievement. In addition, work habits have an effect of its own on achievement. To show the relevance of further dimensions of cultural capital or habitus the following steps have to be taken. At first, parenting practices and other factors relevant for their acquisition in the family of origin have to be identified. Then, differences among the social classes regarding these parenting practices and other relevant factors have to be analyzed. As a good starting point to do this, Lareau's concept of concerted cultivation could be seized and developed further (Lareau, 2002). Generally, concerted cultivation describes a middle classspecific pattern of various parenting practices that enhance skills, knowledge, and attitudes that are relevant in the educational system. Finally, it must be shown that these dimensions of cultural capital and habitus have a significant effect on academic achievement and that they mediate the effect of social origin on academic achievement.

When class-specific parenting practices are analyzed in more depth, the revised theory could be extended by class-specific parenting habitus driving these practices. Parents seem to differ with regard to parenting-relevant beliefs, attitudes, and goals according to their social position (see end of chapter 2.3.1). Therefore, parents with a higher social status also show parenting practices that foster their children's linguistic and cognitive development better than parents with a lower social status do.

There are some limitations concerning the measures for the revised theory when the NEPS SC3 data are used. Data provide only mediocre proxies for some constructs. Due to a lack of better measures, the number of books in the home is used as proxy variable for parental attitudes towards reading and parental passive and active linguistic skills (see chapter 3.2). Moreover, high cultural activities are used as a proxy measure for parents' and students' attitudes towards high culture. More tailored measures would increase the validity of the analyses and the model fit. However, all model fit indicators of the revised model meet the respective thresholds.

Unfortunately, data do not contain measures of high cultural knowledge. Therefore, corresponding hypotheses are not tested. 
The data refers to fifth graders at regular schools in Germany. Hence, the findings are meaningful for this population and not generalizable. Further empirical tests in other stages of the educational system and countries should be conducted to assess generalizability. Moreover, empirical tests on the acquisition of cultural capital and habitus in the relevant age groups should be conducted. For example, empirical findings suggest that language is acquired from a very early age on (see chapter 2.3.1). Hence, corresponding hypotheses (chapter 2.4 .2 ) should be tested using data on early childhood and kindergartens.

The theory of cultural capital presented in this thesis focusses on the explanation of social inequalities in academic achievement. Regarding further exciting research questions, it might be extended to other dimensions of inequality, for example migration background and gender. It could be of further interest whether both, migration- and gender-specific inequalities can be explained by differences in linguistic skills. Drawing on the revised theory, systematic differences between the social classes as well as migrant and native families with regard to conditions of the teaching language acquisition could be analyzed. Moreover, differences between boys and girls in the language acquisition could be explained. Possibly, gender-specific parenting practices can explain differences linguistic skills between boys and girls. Parents might, based on stereotypes, encourage girls more to read than they encourage boys. Hence, girls develop more positive attitudes towards reading than boys do. According to results based on the PIRLS and the PISA study, these differences can actually be observed at the end of primary and lower secondary school (Autorengruppe Bildungsberichterstattung, 2016, p. 95). Therefore, girls read more and develop higher linguistic skills than boys develop.

In summary, research on the theory of cultural capital and habitus still leaves many possibilities for alternatives, expansions or continuations. 


\section{References}

Ajzen, I. (1988). Attitudes, personality and behavior. Milton Keynes, England: Open University Press.

Alba, R. D., Handl, J., \& Müller, W. (1994). Ethnische Ungleichheiten im deutschen Bildungssystem. Kölner Zeitschrift für Soziologie und Sozialpsychologie, 46(2), 209-237.

Aschaffenburg, K., \& Maas, I. (1997). Cultural and educational careers: The dynamics of social reproduction. American Sociological Review, 62(4), 573-587. Retrieved from http://www.jstor.org/stable/2657427

Autorengruppe Bildungsberichterstattung (Ed.) (2016). Bildung in Deutschland 2016: Ein indikatorengestützter Bericht mit einer Analyse zu Bildung und Migration. Bielefeld, Germany: W. Bertelsmann Verlag. Retrieved from https://www.wbv.de/artikel/6001820e https://doi.org/10.3278/6001820ew

Baker, D., Esmer, Y., Lenhardt, G., \& Meyer, J. (1985). Effects of immigrant workers on educational stratification in Germany. Sociology of Education, 58, 213-227.

Baker, L., \& Wigfield, A. (1999). Dimensions of children's motivation for reading and their relations to reading activity and reading achievement. Reading Research Quarterly, 34(4), 452477. Retrieved from http://www.jstor.org/stable/748216

Bandura, A., Barbaranelli, C., Caprara, G. V., \& Pastorelli, C. (1996). Multifaceted impact of selfefficacy beliefs on academic functioning. Child Development, 67(3), 1206-1222. https://doi.org/10.2307/1131888

Baumert, J., Klieme, E., Neubrand, M., Prenzel, M., Schiefele, U., Schneider, W., ... Weiß, M. (Eds.) (2001). PISA 2000: Basiskompetenzen von Schülerinnen und Schülern im internationalen Vergleich. Opladen, Germany: Leske + Budrich.

Baumert, J., \& Schümer, G. (2001). Familiäre Lebensverhältnisse, Bildungsbeteiligung und Kompetenzerwerb. In J. Baumert, E. Klieme, M. Neubrand, M. Prenzel, U. Schiefele, W. Schneider, ... M. Weiß (Eds.), PISA 2000: Basiskompetenzen von Schülerinnen und Schülern im internationalen Vergleich (pp. 323-410). Opladen, Germany: Leske + Budrich.

Becker, B. (2010). The transfer of cultural knowledge in the early childhood: Social and ethnic disparities and the mediating role of familial activities. European Sociological Review, 26(1), 17-29. https://doi.org/10.1093/esr/jcn081

Bendix, R. (Ed.) (1966). Class, status, and power: Social stratification in comparative perspective. New York, NY: Free Press.

Blatt, I., Frahm, S., \& Jarsinski, S. (2013). NEPS technical report for orthography - Scaling results of Starting Cohort 3 in fifth grade (NEPS Working Paper No. 21). Bamberg: University of Bamberg, National Educational Panel Study. Retrieved from https://www.nepsdata.de/Portals/0/Working\%20Papers/WP_XXI.pdf 
Blossfeld, H.-P., Roßbach, H.-G., \& Maurice, J. von (Eds.) (2011). Education as a lifelong process: The German National Educational Panel Study (NEPS). Zeitschrift Für Erziehungswissenschaft [Special issue], 14. Wiesbaden, Germany: VS Verlag für Sozialwissenschaften.

Blossfeld, H.-P., \& Shavit, Y. (1993). Dauerhafte Ungleichheiten. Zeitschrift für Pädagogik, 39(1), 25-52. Retrieved from https://www.pe-

docs.de/volltexte/2017/11164/pdf/ZfPaed_1993_1_Blossfeld_Shavit_Dauerhafte_Ungleichheiten.pdf

Bong, M., \& Skaalvik, E. M. (2003). Academic self-concept and self-efficacy: How different are they really? Educational Psychology Review, 15(1), 1-40.

Bortz, J., \& Schuster, C. (2010). Statistik für Human- und Sozialwissenschaftler (7th revised edition). Berlin, Germany: Springer.

Bos, W., Tarelli, I., Bremerich-Vos, A., \& Schwippert, K. (Eds.) (2012). IGLU 2011: Lesekompetenzen von Grundschulkindern in Deutschland im internationalen Vergleich. Münster, Germany: Waxmann.

Bourdieu, P. (1977a). Cultural reproduction and social reproduction. In J. Karabel \& A.H. Halsey (Eds.), Power and ideology in education. New York, NY: Oxford University Press.

Bourdieu, P. (1977b). Outline of a theory of practice. New York, NY: Cambridge University Press.

Bourdieu, P. (1984). Distinction: A social critique of the judgement of taste. Cambridge, MA: Harvard University Press.

Bourdieu, P. (1986). The forms of capital. In J. G. Richardson (Ed.), Handbook of theory and research for the sociology of education (pp. 241-258). New York, NY: Greenwood Press.

Bourdieu, P. (1987). Die feinen Unterschiede: Kritik der gesellschaftlichen Urteilskraft. Frankfurt am Main, Germany: Suhrkamp.

Bourdieu, P. (1990). The logic of practice. Cambridge, England: Polity Press.

Bourdieu, P., \& Passeron, J.-C. (1971). Die Illusion der Chancengleichheit. Untersuchungen zur Soziologie des Bildungswesens am Beispiel Frankreichs. Stuttgart, Germany: Klett.

Bourdieu, P., \& Passeron, J.-C. (1977). Reproduction in education, society and culture. London, England: Sage Publications.

Bradbury, B., \& Corak, M. (2015). Too many children left behind: The U.S. achievement gap in comparative perspective. New York, NY: Russell Sage Foundation.

Brody, G. H., Flor, D. L., \& Gibson, N. M. (1999). Linking maternal efficacy beliefs, developmental goals, parenting practices, and child competence in rural single-parent African American families. Child Development, 70(5), 1197-1208. https://doi.org/10.1111/1467-8624.00087 
Buchholz, S., \& Pratter, M. (2017). Wer profitiert von alternativen Bildungswegen? Alles eine Frage des Blickwinkels! Kölner Zeitschrift Für Soziologie Und Sozialpsychologie, 69(3), 409435. https://doi.org/10.1007/s11577-017-0484-8

Bus, A. G., van IJzendoorn, M. H., \& Pellegrini, A. D. (1995). Joint book reading makes for success in learning to read: A meta-analysis on intergenerational transmission of literacy. Review of Educational Research, 65(1), 1-21. https://doi.org/10.3102/00346543065001001

Calsyn, R. J., \& Kenny, D. A. (1977). Self-concept of ability and perceived evaluation of others: Cause or effect of academic achievement? Journal of Educational Psychology, 69(2), 136145. https://doi.org/10.1037/0022-0663.69.2.136

Cheung, M. W.-L. (2009). Comparison of methods for constructing confidence intervals of standardized indirect effects. Behavior Research Methods, 41(2), 425-438.

Cheung, S. Y., \& Andersen, R. (2003). Time to read: Family resources and educational outcomes in Britain. Journal of Comparative Family Studies, 34(3), 413-433.

https://doi.org/10.3138/jcfs.34.3.413

Cohen, J., Cohen, P., West, S. G., \& Aiken, L. S. (2015). Applied multiple regression/correlation analysis for the behavioral sciences (3rd edition). New York, NY: Routledge.

Crook, C. J. (1997). The dimensionality of stratification-related cultural practices in Australia. Journal of Sociology, 33(2), 226-238. https://doi.org/10.1177/144078339703300207

De Graaf, N. D., de Graaf, P. M., \& Kraaykamp, G. (2000). Parental cultural capital and educational attainment in the Netherlands: A refinement of the cultural capital perspective. Sociology of Education, 73(2), 92-111.

Demir-Lira, Ö. E., Applebaum, L. R., Goldin-Meadow, S., \& Levine, S. C. (2019). Parents' early book reading to children: Relation to children's later language and literacy outcomes controlling for other parent language input. Developmental Science, 22(3), e12764. https://doi.org/10.1111/desc.12764

Diefenbach, H. (2002). Bildungsbeteiligung und Berufseinmündung von Kindern und Jugendlichen aus Migrantenfamilien: Eine Fortschreibung der Daten des Sozio-ökonomischen Panels. In H. Diefenbach, G. Renner, \& B. Schulte (Eds.), Migration und die europäische Integration: Herausforderungen für die Kinder- und Jugendhilfe (pp. 9-70). München, Germany: Verlag Deutsches Jugendinstitut.

Diefenbach, H., Renner, G., \& Schulte, B. (Eds.) (2002). Migration und die europäische Integration: Herausforderungen für die Kinder- und Jugendhilfe. München, Germany: Verlag Deutsches Jugendinstitut.

DiMaggio, P. (1982). Cultural capital and school success: The impact of status culture participation on the grades of U.S. High school students. American Sociological Review, 47(2), 189201. 
DiMaggio, P., \& Mohr, J. (1985). Cultural capital, educational attainment, and marital selection. American Journal of Sociology, 90(6), 1231-1261. Retrieved from http://www.jstor.org/stable/2779635

Drechsler, J. (2015). Multiple imputation of multilevel missing data: Rigor versus simplicity. Journal of Educational and Behavioral Statistics, 40(1), 69-95. https://doi.org/10.3102/1076998614563393

Dumais, S. A. (2002). Cultural capital, gender, and school success: The role of habitus. Sociology of Education, 75(1), 44-68. https://doi.org/10.2307/3090253

Edgerton, J., Roberts, L., \& Peter, T. (2013). Disparities in academic achievement: Assessing the role of habitus and practice. Social Indicators Research, 114(2), 303-322. Retrieved from https://www.jstor.org/stable/24720249

Farkas, G., Grobe, R. P., Sheehan, D., \& Shuan, Y. (1990). Cultural resources and school success: Gender, ethnicity, and poverty groups within an urban school district. American Sociological Review, 55(1), 127-142. Retrieved from http://www.jstor.org/stable/2095708

Fend, H., Knörzer, W., Nagl, W., Specht, W., \& Väth-Szusdziara, R. (1973). Sozialisationseffekte unterschiedlicher Schulformen. Zeitschrift Für Pädagogik, 19(6), 887-903.

Frahm, S., Goy, M., Kowalski, K., Sixt, M., Strietholt, R., Blatt, I., . . Kanders, M. (2011). Transition and development from lower secondary to upper secondary school. Zeitschrift für Erziehungswissenschaft [Special issue], 14, 217-232. https://doi.org/10.1007/s11618-0110188-1

Friedrichs, J., Mayer, K. U., \& Lepsius, R. (Eds.) (1998). Die Diagnosefähigkeit der Soziologie. Kölner Zeitschrift Für Soziologie Und Sozialpsychologie [Special issue], 38. Opladen, Germany: Westdeutscher Verlag.

Gaddis, S. M. (2013). The influence of habitus in the relationship between cultural capital and academic achievement. Social Science Research, 42(1), 1-13.

https://doi.org/10.1016/j.ssresearch.2012.08.002

Gärtig, A.-K., Plewnia, A., \& Rothe, A. (2010). Wie Menschen in Deutschland über Sprache denken: Ergebnisse einer bundesweiten Repräsentativerhebung zu aktuellen Spracheinstellungen (Arbeitspapiere und Materialien zur deutschen Sprache No. 40). Mannheim. Retrieved from https://ids-pub.bsz-bw.de/frontdoor/index/index/year/2013/docld/560

Gniewosz, B., Eccles, J. S., \& Noack, P. (2015). Early adolescents' development of academic selfconcept and intrinsic task value: The role of contextual feedback. Journal of Research on Adolescence, 25(3), 459-473. https://doi.org/10.1111/jora.12140

Goldthorpe, J. H. (2007). >>Cultural capital<<: Some critical observations. In S. Scherer, R. Pollak, G. Otte, \& M. Gangl (Eds.), From origin to destination: Trends and mechanisms in social stratification research: Essays in honour of Walter Müller (pp. 78-101). Frankfurt am Main, Germany: Campus Verlag. 
Goßmann, F. (2018). Measuring cultural capital in the NEPS (NEPS Survey Paper No. 48). Bamberg, Germany: Leibniz Institute for Educational Trajectories, National Educational Panel Study. Retrieved from https://www.neps-data.de/Portals/0/Survey\%20Papers/SP_XLVIII.pdf

Goßmann, F., \& Mätzke, G. (2019). The cultural knowledge measurement in the NEPS (NEPS Survey Paper No. 55). Bamberg, Germany: Leibniz Institute for Educational Trajectories, National Educational Panel Study. Retrieved from https://www.neps-data.de/Portals/0/Survey\%20Papers/SP_LV.pdf

Graham, J. W., Olchowski, A. E., \& Gilreath, T. D. (2007). How many imputations are really needed?: Some practical clarifications of multiple imputation theory. Prevention Science, 8(3), 206-213. https://doi.org/10.1007/s11121-007-0070-9

Grgic, M., \& Bayer, M. (2015). Eltern und Geschwister als Bildungsressourcen. Zeitschrift für Familienforschung, 27(2), 173-192. Retrieved from https://www.ssoar.info/ssoar/handle/document/44545

Guay, F., Marsh, H. W., \& Boivin, M. (2003). Academic self-concept and academic achievement: Developmental perspectives on their causal ordering. Journal of Educational Psychology, 95(1), 124-136. https://doi.org/10.1037/0022-0663.95.1.124

Guay, F., \& Vallerand, R. J. (1996/1997). Social context, student's motivation, and academic achievement: Toward a process model. Social Psychology of Education, 1(3), 211-233.

Guo, G., \& Stearns, E. (2002). The social influences on the realization of genetic potential for intellectual development. Social Forces, 80(3), 881-910. https://doi.org/10.1353/sof.2002.0007

Haller, A. O. (1968). On the concept of aspiration. Rural Sociology, 33(4), 484-487.

Hart, B., \& Risley, T. R. (1995). Meaningful differences in the everyday experience of young American children. Baltimore, MD: P.H. Brookes.

Heinze, A., Herwartz-Emden, L., \& Reiss, K. (2007). Mathematikkenntnisse und sprachliche Kompetenz bei Kindern mit Migrationshintergrund zu Beginn der Grundschulzeit. Zeitschrift Für Pädagogik, 53(4), 562-581.

Helbig, M., \& Morar, T. (2017). Warum Lehrkräfte sozial ungleich bewerten: Ein Plädoyer für die Etablierung tertiärer Herkunftseffekte im werterwartungstheoretischen Standardmodell der Bildungsforschung (Discussion Paper No. P 2017-005). Berlin, Germany: WZB Berlin Social Science Center. Retrieved from https://bibliothek.wzb.eu/pdf/2017/p17-005.pdf

Henninges, M., Traini, C., \& Kleinert, C. (2019). Tracking and sorting in the German educational system (LIfBi Working Papers No. 83). Bamberg, Germany: Leibniz Institute for Educational Trajectories. Retrieved from https://www.neps-data.de/Portals/0/Working\%20Papers/WP_LXXXIII.pdf

Hippel, P. T. von (2007). Regression with missing Ys: An improved strategy for analyzing multiply-imputed data. Sociological Methodology, 37, 83-117. 
Hoff, E. (2006). How social contexts support and shape language development. Developmental Review, 26, 55-88.

Hoff, E., Laursen, B., \& Tardif, T. (2002). Socioeconomic status and parenting. In M. H. Bornstein (Ed.), Handbook of parenting (2nd ed., pp. 231-252). Mahwah, NJ: Erlbaum.

Hooper, D., Coughlan, J., \& Mullen, M. (2008). Structural equation modelling: Guidelines for determining model fit. Electronic Journal of Business Research Methods, 6(1), 53-60.

Hu, L.-t., \& Bentler, P. M. (1999). Cutoff criteria for fit indexes in covariance structure analysis: Conventional criteria versus new alternatives. Structural Equation Modeling: A Multidisciplinary Journal, 6(1), 1-55.

Hurrelmann, K. (2008). Handbuch Sozialisationsforschung (7th revised edition). Weinheim: Beltz.

Hyman, H. H. (1966). The value systems of different classes: A social psychological contribution to the analysis of stratification. In R. Bendix (Ed.), Class, status, and power: Social stratification in comparative perspective (pp. 488-499). New York, NY: Free Press.

Jæger, M. M. (2011). Does cultural capital really affect academic achievement?: New evidence from combined sibling and panel data. Sociology of Education, 84(4), 281-298. https://doi.org/10.1177/0038040711417010

Jæger, M. M., \& Breen, R. (2016). A dynamic model of cultural reproduction. American Journal of Sociology, 121(4), 1079-1115. https://doi.org/10.1086/684012

Jorgensen, T. D. (2019). Useful tools for structural equation modeling. Retrieved from https://cran.r-project.org/web/packages/semTools/semTools.pdf

Karabel, J., \& Halsey, A.H. (Eds.) (1977). Power and ideology in education. New York, NY: Oxford University Press.

Katsillis, J., \& Rubinson, R. (1990). Cultural capital, student achievement, and educational reproduction: The case of Greece. American Sociological Review, 55(2), 270. https://doi.org/10.2307/2095632

Kingston, P. W. (2001). The unfulfilled promise of cultural capital theory. Sociology of Education, 74, 88-99. https://doi.org/10.2307/2673255

Kline, R. B. (2011). Principles and practice of structural equation modeling (3rd edition). New York, NY: Guilford Press.

Lamont, M., \& Lareau, A. (1988). Cultural capital: Allusions, gaps and glissandos in recent theoretical developments. Sociological Theory, 6(2), 153-168. Retrieved from https://doi.org/10.2307/202113

Lareau, A. (2002). Invisible inequality: Social class and childrearing in black families and white families. American Sociological Review, 67(5), 747-776. https://doi.org/10.2307/3088916 
Lareau, A. (2011). Unequal childhoods: Class, race, and family life (2nd edition). Berkeley, CA: University of California Press.

Lareau, A., \& Horvat, E. M. (1999). Moments of social inclusion and exclusion: Race, class, and cultural capital in family-school relationships. Sociology of Education, 72(1), 37-53. https://doi.org/10.2307/2673185

Lareau, A., \& Weininger, E. B. (2003). Cultural capital in educational research: A critical assessment. Theory and Society, 32(5-6), 567-606. https://doi.org/10.1023/B:RYSO.0000004951.04408.b0

Leibniz Institute for Educational Trajectories, National Educational Panel Study (2018). Information on competence testing: NEPS Starting Cohort 3 - grade 5. Bamberg, Germany. Retrieved from https://www.neps-data.de/Portals/0/NEPS/Datenzentrum/Forschungsdaten/SC3/1-0-0/NEPS_SC3_Competences_W1_en.pdf

Levene, H. (1960). Robust tests for equality of variances. In I. Olkin (Ed.), Contributions to probability and statistics (pp. 278-292). Palo Alto, CA: Stanford University Press.

Linberg, T., Schneider, T., Waldfogel, J., \& Wang, Y. (2019). Socioeconomic status gaps in child cognitive development in Germany and the United States. Social Science Research, 79, 131.

Little, R. J. A. (1988). A test of missing completely at random for multivariate data with missing values. Journal of the American Statistical Association, 83(404), 1198-1202.

MacCallum, R. C., Browne, M. W., \& Sugawara, H. M. (1996). Power analysis and determination of sample size for covariance structure modeling. Psychological Methods, 1(2), 130-149.

MacKinnon, D. P., Fairchild, A. J., \& Fritz, M. S. (2007). Mediation analysis. Annual Review of Psychology, 58, 593-614. https://doi.org/10.1146/annurev.psych.58.110405.085542

Maehler, D. B., Massing, N., Helmschrott, S., Rammstedt, B., Staudinger, U. M., \& Wolf, C. (2013). Grundlegende Kompetenzen in verschiedenen Bevölkerungsgruppen. In B. Rammstedt (Ed.), Grundlegende Kompetenzen Erwachsener im internationalen Vergleich: Ergebnisse von PIAAC 2012 (pp. 77-126). Münster, Germany: Waxmann.

McDill, E. L., \& Coleman, J. (1965). Family and peer influences in college plans of high school students. Sociology of Education, 38(2), 112-126. https://doi.org/10.2307/2112194

McElvany, N., Kortenbruck, M., \& Becker, M. (2008). Lesekompetenz und Lesemotivation. Zeitschrift Für Pädagogische Psychologie, 22(34), 207-219. https://doi.org/10.1024/10100652.22.34.207

Merton, R. K. (1968). The Matthew effect in science. Science, 159(3810), 56-63. https://doi.org/10.1126/science.159.3810.56 
Mikus, K., Tieben, N., \& Schober, P. S. (2020). Children's conversion of cultural capital into educational success: The symbolic and skill-generating functions of cultural capital. British Journal of Sociology of Education, 41(2), 197-217. https://doi.org/10.1080/01425692.2019.1677454

Möller, J., \& Bonerad, E.-M. (2007). Fragebogen zur habituellen Lesemotivation. Psychologie in Erziehung Und Unterricht, 54(1), 259-267.

Morris, T. P., White, I. R., \& Royston, P. (2014). Tuning multiple imputation by predictive mean matching and local residual draws. BMC Medical Research Methodology, 14(1), 75. https://doi.org/10.1186/1471-2288-14-75

Mudiappa, M. (2014). Ausmaß und Bedingtheit von kulturellem Kapital und seine Auswirkungen im Kontext Familie und Schule. Berlin, Germany: Logos Verlag.

Müller, H.-P. (2014). Pierre Bourdieu: Eine systematische Einführung. Berlin, Germany: Suhrkamp.

Müller, W. (1998). Erwartete und unerwartete Folgen der Bildungsexpansion. Kölner Zeitschrift für Soziologie und Sozialpsychologie [Special issue], 38, 81-112.

Münch, R. (2004). Gesellschaftstheorie. Frankfurt am Main, Germany: Campus Verlag.

Nash, R. (1990). Bourdieu on education and social and cultural reproduction. British Journal of Sociology of Education, 11(4), 431-447. Retrieved from http://www.jstor.org/stable/1392877

Oberski, D. (2016). Complex survey structural equation modeling (SEM). Retrieved from https://cran.r-project.org/web/packages/lavaan.survey/lavaan.survey.pdf

Olkin, I. (Ed.) (1960). Contributions to probability and statistics. Palo Alto, CA: Stanford University Press.

Opp, K.-D. (2005). Methodologie der Sozialwissenschaften: Einführung in Probleme ihrer Theorienbildung und praktischen Anwendung (6th edition). Wiesbaden, Germany: VS Verlag für Sozialwissenschaften.

Paulus, W., \& Blossfeld, H.-P. (2007). Schichtspezifische Präferenzen oder sozioökonomisches Entscheidungskalkül? Zur Rolle elterlicher Bildungsaspirationen im Entscheidungsprozess beim Übergang von der Grundschule in die Sekundarstufe. Zeitschrift Für Pädagogik, 53(4), 491-508. Retrieved from http://www.pedocs.de/volltexte/2011/4407/

Pohl, S., \& Carstensen, C. H. (2012). NEPS technical report - Scaling the data of the competence tests (NEPS Working Paper No. 14). Bamberg: University of Bamberg, National Educational Panel Study. Retrieved from https://www.neps-data.de/Portals/0/Working\%20Papers/WP_XIV.pdf

Pohl, S., Haberkorn, K., Hardt, K., \& Wiegand, E. (2012). NEPS Technical Report of Reading Scaling results of Starting Cohort 3 in fifth grade (NEPS Working Paper No. 15). Bamberg: 
University of Bamberg, National Educational Panel Study. Retrieved from https://www.neps-data.de/Portals/0/Working\%20Papers/WP_XV.pdf

Raghunathan, T. W., Lepkowski, J. M., van Hoewyk, J., \& Solenberger, P. (2001). A multivariate technique for multiply imputing missing values using a sequence of regression models. Survey Methodology, 27, 85-95.

Rammstedt, B. (Ed.) (2013). Grundlegende Kompetenzen Erwachsener im internationalen Vergleich: Ergebnisse von PIAAC 2012. Münster, Germany: Waxmann.

Richardson, J. G. (Ed.) (1986). Handbook of theory and research for the sociology of education. New York, NY: Greenwood Press.

Rosenberg, M., \& Turner, R. H. (Eds.) (1981). Sociological perspectives. New York, NY: Basic Books.

Rössel, J., \& Beckert-Zieglschmid, C. (2002). Die Reproduktion kulturellen Kapitals. Zeitschrift Für Soziologie, 31(6), 497-513.

Rubin, D. B. (1976). Inference and missing data. Biometrika, 63, 581-592.

Rubin, D. B. (1987). Multiple imputation for nonresponse in surveys. New York, NY: John Wiley \& Sons.

Scherer, S., Pollak, R., Otte, G., \& Gangl, M. (Eds.) (2007). From origin to destination: Trends and mechanisms in social stratification research: Essays in honour of Walter Müller. Frankfurt am Main, Germany: Campus Verlag.

Schindler, S., \& Lörz, M. (2011). Mechanisms of social inequality development: Primary and secondary effects in the transition to tertiary education between 1976 and 2005. European Sociological Review, 28(5), 647-660. https://doi.org/10.1093/esr/jcr032

Sewell, W. H., Haller, A. O., \& Portes, A. (1969). The educational and early occupational attainment process. American Sociological Review, 34(1), 82-92.

https://doi.org/10.2307/2092789

Singer, E. (1981). Reference groups and social evaluations. In M. Rosenberg \& R. H. Turner (Eds.), Sociological perspectives (pp. 66-93). New York, NY: Basic Books.

Sobel, M. E. (1982). Asymptotic confidence intervals for indirect effects in structural equation models. Sociological Methodology, 13, 290. https://doi.org/10.2307/270723

Steinhauer, H. W., \& Zinn, S. (2016). NEPS technical report for weighting: Weighting the sample of Starting Cohort 3 of the National Educational Panel Study (waves 1 to 3) (NEPS Working Paper No. 63). Bamberg, Germany: Leibniz Institute for Educational Trajectories, National Educational Panel Study. Retrieved from https://www.neps-data.de/Portals/0/Working\%20Papers/WP_LXIII.pdf

Stocké, V., Blossfeld, H.-P., Hoenig, K., \& Sixt, M. (2011). Social inequality and educational decisions in the life course. Zeitschrift für Erziehungswissenschaft [Special issue], 14, 103-119. https://doi.org/10.1007/s11618-011-0193-4 
Sullivan, A. (2000). Cultural capital, rational choice, and educational inequalities (Doctoral dissertation): Oxford University, Oxford, England. Retrieved from https://www.researchgate.net/profile/Alice_Sullivan2/publication/265481051_Cultural_Capital_Rational_Choice_and_Educational_Inequalities/links/54e37e280cf282dbed6bef27/CulturalCapital-Rational-Choice-and-Educational-Inequalities.pdf

Sullivan, A. (2001). Cultural capital and educational attainment. Sociology, 35(4), 893-912.

Sullivan, A. (2002). Bourdieu and education: How useful is Bourdieu's theory for researchers? The Netherlands' Journal of Social Sciences, 38(2), 144-166.

Sullivan, T. R., Salter, A. B., Ryan, P., \& Lee, K. J. (2015). Bias and precision of the "multiple imputation, then deletion" method for dealing with missing outcome data. American Journal of Epidemiology, 182, 528-534.

Swartz, D. (1997). Culture \& power: The sociology of Pierre Bourdieu. Chicago, IL: University of Chicago Press.

Teman, E. D. (2012). The performance of multiple imputation and full information maximum likelihood for missing ordinal data in structural equation models (Doctoral dissertation No. UMI 3555133). Retrieved from ProQuest Dissertation and Theses database https://search.proquest.com/docview/1318673207?accountid=8485

Van Buuren, S. (2007). Multiple imputation of discrete and continuous data by fully conditional specification. Statistical Methods in Medical Research, 16, 219-242.

Weber, M. (1978). Economy and society: An outline of interpretive sociology (G. Roth \& C. Wittich, Trans.). Berkeley, CA: University of California Press.

Weinert, S., Ebert, S., \& Dubowy, M. (2010). Kompetenzen und soziale Disparitäten im Vorschulalter. Zeitschrift Für Grundschulforschung, 3(1), 32-45.

Wendt, H., Stubbe, T. C., \& Schwippert, K. (2012). Soziale Herkunft und Lesekompetenzen von Schülerinnen und Schülern. In W. Bos, I. Tarelli, A. Bremerich-Vos, \& K. Schwippert (Eds.), IGLU 2011: Lesekompetenzen von Grundschulkindern in Deutschland im internationalen Vergleich (pp. 175-190). Münster, Germany: Waxmann.

White, I. R., Royston, P., \& Wood, A. M. (2011). Multiple imputation using chained equations: Issues and guidance for practice. Statistics in Medicine, 30(4), 377-399. https://doi.org/10.1002/sim.4067

Wildhagen, T. (2009). Why does cultural capital matter for high school academic performance?: An empirical assessment of teacher-selection and self-selection mechanisms as explanations of the cultural capital effect. Sociological Quarterly, 50(1), 173-200. Retrieved from http://search.ebscohost.com/login.aspx?di-

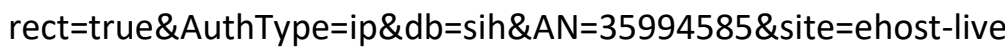


Zabal, A., Martin, S., Klaukien, A., Rammstedt, B., Baumert, J., \& Klieme, E. (2013). Grundlegende Kompetenzen der erwachsenen Bevölkerung in Deutschland im internationalen Vergleich. In B. Rammstedt (Ed.), Grundlegende Kompetenzen Erwachsener im internationalen Vergleich: Ergebnisse von PIAAC 2012 (pp. 31-76). Münster, Germany: Waxmann. 


\section{Appendix}

\section{A. List of abbreviations}

\begin{tabular}{|c|c|}
\hline ANOVA & Analyses of variance \\
\hline BiKS & $\begin{array}{l}\text { Bildungsprozesse, Kompetenzentwicklung und Selektionsentscheidun- } \\
\text { gen im Vor- und Grundschulalter (Educational processes, competence } \\
\text { development and selection decisions in preschool- and school age) }\end{array}$ \\
\hline BMBF & $\begin{array}{l}\text { Bundesministerium für Bildung und Forschung (German Federal Minis- } \\
\text { try of Education and Research) }\end{array}$ \\
\hline CATI & Computer assisted telephone interview \\
\hline CFI & Comparative fit index \\
\hline EGP & Erikson-Goldthorpe-Portocarero class scheme \\
\hline FIML & Full information maximum likelihood method \\
\hline GCSE & General Certificate of Secondary Education \\
\hline ISEI & International Socio-Economic Index of Occupational Status \\
\hline LIfBi & $\begin{array}{l}\text { Leibniz-Institut für Bildungsverläufe (Leibniz Institute for Educational } \\
\text { Trajectories) }\end{array}$ \\
\hline MAR & Missing at Random \\
\hline MCAR & Missing Completely at Random \\
\hline MICE & Multiple imputation by chained equations \\
\hline MID & Multiple imputation, then deletion approach \\
\hline ML & Maximum likelihood \\
\hline NELS & National Education Longitudinal Study \\
\hline NEPS & National Educational Panel Study \\
\hline NMAR & Not Missing at Random \\
\hline OLS & Ordinary least squares \\
\hline PAPI & Paper and pencil interview \\
\hline PIAAC & Program for the International Assessment of Adult Competencies \\
\hline PIRLS & Progress in International Reading Literacy Study \\
\hline PISA & Program for International Student Assessment \\
\hline PMM & Predictive mean matching \\
\hline RMSEA & Root mean square error of approximation \\
\hline
\end{tabular}


SC3

SC4

SES

SRMR

WLE
Starting Cohort 3 of the National Educational Panel Study

Starting Cohort 4 of the National Educational Panel Study

Socioeconomic status

Standardized root mean square residual

Weighted maximum likelihood estimates 


\section{B. Tables}

Table 14: Cross table of the highest social class position (EGP) and the highest formal education of the parents

\begin{tabular}{|c|c|c|c|c|c|c|}
\hline \multirow[b]{2}{*}{$\begin{array}{l}\text { Highest social } \\
\text { class position } \\
\text { (EGP) }\end{array}$} & \multicolumn{5}{|c|}{ Highest formal education } & \multirow[b]{2}{*}{ Total } \\
\hline & $\begin{array}{l}\text { Lower } \\
\text { secondary }\end{array}$ & $\begin{array}{l}\text { Intermediate } \\
\text { secondary }\end{array}$ & $\begin{array}{l}\text { Higher } \\
\text { secondary }\end{array}$ & $\begin{array}{l}\text { Lower } \\
\text { tertiary }\end{array}$ & $\begin{array}{l}\text { Higher } \\
\text { tertiary }\end{array}$ & \\
\hline $\begin{array}{l}\text { Higher service } \\
\text { class (EGP I) }\end{array}$ & $\begin{array}{l}38 \\
3.19\end{array}$ & $\begin{array}{l}186 \\
15.63\end{array}$ & $\begin{array}{l}224 \\
18.82\end{array}$ & $\begin{array}{l}201 \\
16.89\end{array}$ & $\begin{array}{l}541 \\
45.46\end{array}$ & $\begin{array}{l}1,190 \\
100.00\end{array}$ \\
\hline $\begin{array}{l}\text { Lower service } \\
\text { class (EGP II) }\end{array}$ & $\begin{array}{l}52 \\
5.15\end{array}$ & $\begin{array}{l}365 \\
36.17\end{array}$ & $\begin{array}{l}253 \\
25.07\end{array}$ & $\begin{array}{l}117 \\
11.60\end{array}$ & $\begin{array}{l}222 \\
22.00\end{array}$ & $\begin{array}{l}1,009 \\
100.00\end{array}$ \\
\hline $\begin{array}{l}\text { Intermediate } \\
\text { class (EGP III, V) }\end{array}$ & $\begin{array}{l}135 \\
14.80\end{array}$ & $\begin{array}{l}485 \\
53.18\end{array}$ & $\begin{array}{l}215 \\
23.57\end{array}$ & $\begin{array}{l}37 \\
4.06\end{array}$ & $\begin{array}{l}40 \\
4.39\end{array}$ & $\begin{array}{l}912 \\
100.00\end{array}$ \\
\hline $\begin{array}{l}\text { Petty-bourgeoisie } \\
\text { (EGP IV) }\end{array}$ & $\begin{array}{l}19 \\
20.43\end{array}$ & $\begin{array}{l}37 \\
39.78\end{array}$ & $\begin{array}{l}26 \\
27.96\end{array}$ & $\begin{array}{l}5 \\
5.38\end{array}$ & $\begin{array}{l}6 \\
6.45\end{array}$ & $\begin{array}{l}93 \\
100.00\end{array}$ \\
\hline $\begin{array}{l}\text { Manual worker } \\
\text { (EGP VI, VII) }\end{array}$ & $\begin{array}{l}171 \\
43.62\end{array}$ & $\begin{array}{l}157 \\
40.05\end{array}$ & $\begin{array}{l}58 \\
14.80\end{array}$ & $\begin{array}{l}3 \\
0.77\end{array}$ & $\begin{array}{l}3 \\
0.77\end{array}$ & $\begin{array}{l}392 \\
100.00\end{array}$ \\
\hline Total & $\begin{array}{l}415 \\
11.54\end{array}$ & $\begin{array}{l}1,230 \\
34.20\end{array}$ & $\begin{array}{l}776 \\
21.58\end{array}$ & $\begin{array}{l}363 \\
10.09\end{array}$ & $\begin{array}{l}812 \\
22.58\end{array}$ & $\begin{array}{l}3,596 \\
100.00\end{array}$ \\
\hline
\end{tabular}

Source: own calculations based on NEPS SC3 7.0.0

Notes: The table shows absolute values (first row) and the relative share of frequencies within the rows (second row). 
Table 15: Standardized residual covariances - common model - single-group analysis

\begin{tabular}{|c|c|c|c|c|c|c|c|c|c|c|c|c|c|c|c|}
\hline & $x 2$ & x3 & $x 4$ & $x 6$ & $\times 9$ & $\mathrm{x} 1.2$ & $x 1.3$ & $\mathrm{x} 1.4$ & x1.5 & c1 & c2 & c3 & c4 & c5.2 & c5.3 \\
\hline$x 2$ & 0.000 & & & & & & & & & & & & & & \\
\hline x3 & 1.169 & 1.211 & & & & & & & & & & & & & \\
\hline $\mathrm{x} 4$ & 0.000 & -0.899 & 0.000 & & & & & & & & & & & & \\
\hline x6 & 1.588 & 0.989 & -0.308 & -0.247 & & & & & & & & & & & \\
\hline x9 & 0.021 & -2.272 & -0.165 & 0.660 & -0.196 & & & & & & & & & & \\
\hline $\mathrm{x} 1.2$ & 0.000 & 0.000 & 0.000 & 0.000 & 0.000 & 0.000 & & & & & & & & & \\
\hline $\mathrm{x} 1.3$ & 0.000 & 0.000 & 0.000 & 0.000 & 0.000 & 0.000 & 0.000 & & & & & & & & \\
\hline $\mathrm{x} 1.4$ & 0.000 & 0.000 & 0.000 & 0.000 & 0.000 & 0.000 & 0.000 & 0.000 & & & & & & & \\
\hline $\mathrm{x} 1.5$ & 0.000 & 0.000 & 0.000 & 0.000 & 0.000 & 0.000 & 0.000 & 0.000 & 0.000 & & & & & & \\
\hline c1 & 0.000 & 0.000 & 0.000 & 0.000 & 0.000 & 0.000 & 0.000 & 0.000 & 0.000 & 0.000 & & & & & \\
\hline c2 & -1.128 & -0.979 & -0.351 & -0.219 & -0.249 & 0.000 & 0.000 & 0.000 & 0.000 & 0.000 & 0.000 & & & & \\
\hline c3 & 0.510 & 0.663 & -0.497 & 2.149 & 0.393 & 0.000 & 0.000 & 0.000 & 0.000 & 0.000 & 0.000 & 0.000 & & & \\
\hline c4 & 4.353 & -0.723 & 5.238 & 4.251 & 0.835 & 0.000 & 0.000 & 0.000 & 0.000 & 0.000 & 0.000 & 0.000 & 0.000 & & \\
\hline c5.2 & 4.579 & 2.411 & 3.517 & 5.401 & 1.202 & 0.000 & 0.000 & 0.000 & 0.000 & 0.000 & 0.000 & 0.000 & 0.000 & 0.000 & \\
\hline c5.3 & 0.283 & 1.097 & -0.202 & -0.604 & 0.042 & 0.000 & 0.000 & 0.000 & 0.000 & 0.000 & 0.000 & 0.000 & 0.000 & 0.000 & 0.000 \\
\hline
\end{tabular}

Source: own calculations based on NEPS SC3 7.0.0

Note: bold values $p<0.05$ 
Table 16: Standardized residuals covariances - revised model - single-group analysis

\begin{tabular}{|c|c|c|c|c|c|c|c|c|c|c|c|c|c|c|c|c|c|c|}
\hline & x2 & x3 & $x 4$ & x5 & x6 & $x 7$ & $x 8$ & x9 & x1.2 & x1.3 & x1.4 & x1.5 & c1 & c2 & c3 & c4 & c5.2 & c5.3 \\
\hline$x 2$ & 0.000 & & & & & & & & & & & & & & & & & \\
\hline$x 3$ & 3.065 & 3.091 & & & & & & & & & & & & & & & & \\
\hline$x 4$ & 0.000 & -0.766 & 0.000 & & & & & & & & & & & & & & & \\
\hline$x 5$ & 4.472 & 3.192 & -0.100 & -0.068 & & & & & & & & & & & & & & \\
\hline$x 6$ & 2.380 & 4.085 & 3.313 & -0.059 & -0.049 & & & & & & & & & & & & & \\
\hline$x 7$ & 5.551 & -1.887 & 4.214 & 9.570 & 4.550 & 1.706 & & & & & & & & & & & & \\
\hline$x 8$ & 3.505 & 0.238 & 3.671 & 9.411 & 4.330 & 1.395 & 1.113 & & & & & & & & & & & \\
\hline$x 9$ & 0.293 & -2.465 & -1.576 & 3.099 & 0.364 & -6.205 & -8.613 & -8.231 & & & & & & & & & & \\
\hline $\mathrm{x} 1.2$ & 0.000 & 0.000 & 0.000 & 0.000 & 0.000 & 0.000 & 0.000 & 0.000 & 0.000 & & & & & & & & & \\
\hline $\mathrm{x} 1.3$ & 0.000 & 0.000 & 0.000 & 0.000 & 0.000 & 0.000 & 0.000 & 0.000 & 0.000 & 0.000 & & & & & & & & \\
\hline x1.4 & 0.000 & 0.000 & 0.000 & 0.000 & 0.000 & 0.000 & 0.000 & 0.000 & 0.000 & 0.000 & 0.000 & & & & & & & \\
\hline $\mathrm{x} 1.5$ & 0.000 & 0.000 & 0.000 & 0.000 & 0.000 & 0.000 & 0.000 & 0.000 & 0.000 & 0.000 & 0.000 & 0.000 & & & & & & \\
\hline c1 & 0.000 & 0.000 & 0.000 & 0.000 & 0.000 & 0.000 & 0.000 & 0.000 & 0.000 & 0.000 & 0.000 & 0.000 & 0.000 & & & & & \\
\hline c2 & -1.127 & -0.965 & -0.351 & -0.203 & -0.147 & -0.079 & -0.053 & -0.193 & 0.000 & 0.000 & 0.000 & 0.000 & 0.000 & 0.000 & & & & \\
\hline c3 & 0.510 & 0.660 & -0.496 & 0.959 & 2.146 & 0.227 & 0.267 & 0.266 & 0.000 & 0.000 & 0.000 & 0.000 & 0.000 & 0.000 & 0.000 & & & \\
\hline c4 & 4.351 & -0.725 & 5.235 & 4.641 & 4.248 & 1.277 & 0.988 & 0.891 & 0.000 & 0.000 & 0.000 & 0.000 & 0.000 & 0.000 & 0.000 & 0.000 & & \\
\hline c5.2 & 4.577 & 2.410 & 3.514 & 7.987 & 5.398 & 8.139 & 10.521 & 7.825 & 0.000 & 0.000 & 0.000 & 0.000 & 0.000 & 0.000 & 0.000 & 0.000 & 0.000 & \\
\hline c5.3 & 0.282 & 1.097 & -0.200 & -1.213 & -0.605 & -1.743 & -3.152 & -2.044 & 0.000 & 0.000 & 0.000 & 0.000 & 0.000 & 0.000 & 0.000 & 0.000 & 0.000 & 0.000 \\
\hline
\end{tabular}

Source: own calculations based on NEPS SC3 7.0.0

Note: bold values $p<0.05$ 
Table 17: Standardized residual covariances - revised model - multiple-group path analysis (non-final) - no tracking

\begin{tabular}{|c|c|c|c|c|c|c|c|c|c|c|c|c|c|c|c|c|}
\hline & $x 2$ & x3 & $x 4$ & $x 5$ & $x 6$ & $x 7$ & $x 8$ & $\times 9$ & $x 1.2$ & $x 1.3$ & x1.4 & $x 1.5$ & c1 & c2 & c3 & c4 \\
\hline$x 2$ & 1.471 & & & & & & & & & & & & & & & \\
\hline$x 3$ & -0.159 & -0.163 & & & & & & & & & & & & & & \\
\hline$x 4$ & 1.655 & 1.431 & 2.123 & & & & & & & & & & & & & \\
\hline$x 5$ & 0.753 & -0.101 & 0.939 & 0.651 & & & & & & & & & & & & \\
\hline$x 6$ & 1.414 & 0.932 & 1.434 & 0.046 & 0.005 & & & & & & & & & & & \\
\hline$x 7$ & 3.421 & 0.787 & 3.242 & 3.645 & 2.309 & 3.241 & & & & & & & & & & \\
\hline$x 8$ & 2.226 & 1.697 & 2.280 & 4.243 & 1.802 & 2.635 & 2.525 & & & & & & & & & \\
\hline$x 9$ & 2.678 & 1.637 & 2.182 & 5.319 & 2.896 & 2.782 & 2.459 & 1.754 & & & & & & & & \\
\hline $\mathrm{x} 1.2$ & -0.587 & -0.473 & -0.836 & 0.307 & 0.383 & -0.416 & -0.144 & -0.798 & 0.000 & & & & & & & \\
\hline $\mathrm{x} 1.3$ & 0.406 & -0.432 & -0.136 & -0.750 & 0.367 & 0.019 & -0.981 & -0.264 & 0.000 & 0.000 & & & & & & \\
\hline $\mathrm{x} 1.4$ & 1.456 & -0.687 & 2.333 & 0.913 & -0.159 & 1.580 & 1.069 & 2.095 & 0.000 & 0.000 & 0.000 & & & & & \\
\hline $\mathrm{x} 1.5$ & 0.629 & 1.645 & 0.533 & 1.013 & 1.374 & 0.902 & 1.670 & -0.213 & 0.000 & 0.000 & 0.000 & 0.000 & & & & \\
\hline c1 & -0.289 & 0.135 & -0.182 & -0.614 & -1.345 & -1.090 & -0.671 & 1.997 & 0.000 & 0.000 & 0.000 & 0.000 & 0.000 & & & \\
\hline c2 & -0.621 & -0.642 & -0.692 & -0.633 & -0.753 & -0.484 & -1.623 & -0.826 & 0.000 & 0.000 & 0.000 & 0.000 & 0.000 & 0.000 & & \\
\hline c3 & 0.523 & 0.171 & 0.025 & 0.114 & 1.117 & 0.371 & -0.253 & -0.960 & 0.000 & 0.000 & 0.000 & 0.000 & 0.000 & 0.000 & 0.000 & \\
\hline c4 & 1.813 & 1.990 & 1.992 & 2.915 & 3.181 & 2.373 & 1.672 & 2.024 & 0.000 & 0.000 & 0.000 & 0.000 & 0.000 & 0.000 & 0.000 & 0.000 \\
\hline
\end{tabular}

Source: own calculations based on NEPS SC3 7.0.0

Note: bold values $p<0.05$ 
Table 18: Standardized residual covariances - revised model - multiple-group path analysis (non-final) - lower and intermediate track

\begin{tabular}{|c|c|c|c|c|c|c|c|c|c|c|c|c|c|c|c|c|}
\hline & $\mathbf{x} 2$ & $x 3$ & $x 4$ & x5 & x6 & $x 7$ & $x 8$ & $x 9$ & $\mathrm{x} 1.2$ & x1.3 & $\mathrm{x} 1.4$ & x1.5 & c1 & c2 & c3 & c4 \\
\hline$x 2$ & -3.911 & & & & & & & & & & & & & & & \\
\hline$x 3$ & -3.215 & -1.674 & & & & & & & & & & & & & & \\
\hline$x 4$ & -2.595 & -3.970 & -0.784 & & & & & & & & & & & & & \\
\hline x5 & 2.337 & 2.156 & -0.869 & 3.359 & & & & & & & & & & & & \\
\hline$x 6$ & 0.638 & 2.135 & -0.031 & -4.219 & -5.322 & & & & & & & & & & & \\
\hline x7 & 0.520 & -5.667 & -0.008 & 2.913 & -3.148 & -0.526 & & & & & & & & & & \\
\hline x8 & -0.080 & -3.628 & 0.540 & 3.832 & -3.684 & 0.331 & 0.412 & & & & & & & & & \\
\hline x9 & -0.372 & -5.134 & -2.082 & 1.236 & -0.742 & -0.704 & 0.099 & -2.454 & & & & & & & & \\
\hline$x 1.2$ & 2.094 & 3.679 & 0.505 & -0.676 & 1.813 & -0.060 & -1.245 & 0.627 & 0.000 & & & & & & & \\
\hline$x 1.3$ & -1.146 & -0.361 & 1.234 & 0.574 & -0.730 & 0.280 & 0.727 & -0.755 & 0.000 & 0.000 & & & & & & \\
\hline x1.4 & -1.351 & -1.891 & -1.079 & -0.558 & -2.627 & -0.732 & 0.299 & -0.676 & 0.000 & 0.000 & 0.000 & & & & & \\
\hline$x 1.5$ & -1.886 & -3.886 & -0.631 & -0.611 & -1.716 & -0.706 & 0.239 & -0.563 & 0.000 & 0.000 & 0.000 & 0.000 & & & & \\
\hline c1 & 0.760 & 0.894 & -0.762 & -0.221 & 0.893 & 0.823 & -0.035 & -0.722 & 0.000 & 0.000 & 0.000 & 0.000 & 0.000 & & & \\
\hline c2 & -1.585 & 1.725 & -0.415 & -3.843 & -1.594 & 0.055 & -0.686 & 0.963 & 0.000 & 0.000 & 0.000 & 0.000 & 0.000 & 0.000 & & \\
\hline c3 & -1.379 & -0.271 & -1.114 & 0.698 & 0.693 & 0.189 & 1.211 & 0.280 & 0.000 & 0.000 & 0.000 & 0.000 & 0.000 & 0.000 & 0.000 & \\
\hline c4 & 2.037 & -3.446 & 3.281 & 0.519 & -0.122 & 0.084 & 0.219 & -3.987 & 0.000 & 0.000 & 0.000 & 0.000 & 0.000 & 0.000 & 0.000 & 0.000 \\
\hline
\end{tabular}

Source: own calculations based on NEPS SC3 7.0.0

Note: bold values $p<0.05$ 
Table 19: Standardized residual covariances - revised model - multiple-group path analysis (non-final) - higher track

\begin{tabular}{|c|c|c|c|c|c|c|c|c|c|c|c|c|c|c|c|c|}
\hline & $x 2$ & $x 3$ & $x 4$ & $x 5$ & x6 & $x 7$ & $x 8$ & $\times 9$ & $\mathrm{x} 1.2$ & $\mathrm{x} 1.3$ & $\mathrm{x} 1.4$ & $\mathrm{x} 1.5$ & c1 & c2 & c3 & c4 \\
\hline$x 2$ & 2.556 & & & & & & & & & & & & & & & \\
\hline$x 3$ & 4.400 & 3.023 & & & & & & & & & & & & & & \\
\hline$x 4$ & 1.376 & 1.889 & -1.262 & & & & & & & & & & & & & \\
\hline$x 5$ & 2.030 & -0.384 & -0.023 & -4.525 & & & & & & & & & & & & \\
\hline$x 6$ & 0.934 & 3.276 & 3.333 & 5.206 & 6.172 & & & & & & & & & & & \\
\hline$x 7$ & 1.945 & -0.013 & 0.938 & 4.945 & 3.188 & -1.941 & & & & & & & & & & \\
\hline$x 8$ & 0.040 & 1.303 & 0.153 & 4.407 & 3.279 & -2.174 & -2.305 & & & & & & & & & \\
\hline x9 & 1.335 & 1.461 & 0.390 & 2.656 & 1.441 & -1.724 & -1.924 & 0.821 & & & & & & & & \\
\hline $\mathrm{x} 1.2$ & -2.291 & -2.979 & 0.154 & 0.238 & -2.247 & 0.513 & 1.763 & 0.148 & 0.000 & & & & & & & \\
\hline$x 1.3$ & 0.726 & 0.860 & -0.697 & 0.014 & 0.496 & -0.269 & 0.099 & 0.796 & 0.000 & 0.000 & & & & & & \\
\hline x1.4 & 0.447 & 2.872 & -1.440 & -0.220 & 3.184 & -0.884 & -1.517 & -1.770 & 0.000 & 0.000 & 0.000 & & & & & \\
\hline $\mathrm{x} 1.5$ & 1.460 & 1.771 & 0.137 & -0.129 & 0.507 & -0.554 & -1.951 & 0.857 & 0.000 & 0.000 & 0.000 & 0.000 & & & & \\
\hline c1 & -0.636 & -1.165 & 0.741 & 0.876 & 0.143 & -0.108 & 0.542 & -0.906 & 0.000 & 0.000 & 0.000 & 0.000 & 0.000 & & & \\
\hline c2 & -0.239 & -1.503 & 0.281 & 4.966 & 1.596 & 0.126 & 2.262 & -0.392 & 0.000 & 0.000 & 0.000 & 0.000 & 0.000 & 0.000 & & \\
\hline c3 & 0.396 & 0.582 & -0.795 & -0.505 & 1.172 & -0.521 & -0.743 & 0.872 & 0.000 & 0.000 & 0.000 & 0.000 & 0.000 & 0.000 & 0.000 & \\
\hline c4 & 1.076 & -0.516 & 2.362 & 1.214 & 2.283 & -1.276 & -0.929 & 2.251 & 0.000 & 0.000 & 0.000 & 0.000 & 0.000 & 0.000 & 0.000 & 0.000 \\
\hline
\end{tabular}

Source: own calculations based on NEPS SC3 7.0.0

Note: bold values $p<0.05$ 
Table 20: Standardized residual covariances - revised model - multiple-group path analysis (final) - no tracking

\begin{tabular}{|c|c|c|c|c|c|c|c|c|c|c|c|c|c|c|c|c|}
\hline & $x 2$ & $x 3$ & $x 4$ & $x 5$ & $x 6$ & $x 7$ & $x 8$ & $x 9$ & $x 1.2$ & $x 1.3$ & x1.4 & $x 1.5$ & c1 & c2 & c3 & c4 \\
\hline$x 2$ & 1.471 & & & & & & & & & & & & & & & \\
\hline$x 3$ & -0.159 & -0.163 & & & & & & & & & & & & & & \\
\hline$x 4$ & 1.655 & 1.431 & 2.123 & & & & & & & & & & & & & \\
\hline$x 5$ & 0.753 & -0.101 & 0.939 & 0.651 & & & & & & & & & & & & \\
\hline$x 6$ & 1.414 & 0.932 & 1.434 & 0.046 & 0.005 & & & & & & & & & & & \\
\hline$x 7$ & 3.421 & 0.787 & 3.242 & 3.645 & 2.309 & 3.241 & & & & & & & & & & \\
\hline$x 8$ & 2.226 & 1.697 & 2.280 & 4.243 & 1.802 & 2.635 & 2.525 & & & & & & & & & \\
\hline x9 & 2.853 & 1.655 & 2.738 & 5.308 & 2.860 & 2.860 & 2.581 & 2.533 & & & & & & & & \\
\hline$x 1.2$ & -0.587 & -0.473 & -0.836 & 0.307 & 0.383 & -0.416 & -0.144 & -0.270 & 0.000 & & & & & & & \\
\hline $\mathrm{x} 1.3$ & 0.406 & -0.432 & -0.136 & -0.750 & 0.367 & 0.019 & -0.981 & -0.524 & 0.000 & 0.000 & & & & & & \\
\hline x1.4 & 1.456 & -0.687 & 2.333 & 0.913 & -0.159 & 1.580 & 1.069 & 1.002 & 0.000 & 0.000 & 0.000 & & & & & \\
\hline $\mathrm{x} 1.5$ & 0.629 & 1.645 & 0.533 & 1.013 & 1.374 & 0.902 & 1.670 & 1.082 & 0.000 & 0.000 & 0.000 & 0.000 & & & & \\
\hline c1 & -0.289 & 0.135 & -0.182 & -0.614 & -1.345 & -1.090 & -0.671 & -0.738 & 0.000 & 0.000 & 0.000 & 0.000 & 0.000 & & & \\
\hline c2 & -0.621 & -0.642 & -0.692 & -0.633 & -0.753 & -0.484 & -1.623 & -1.095 & 0.000 & 0.000 & 0.000 & 0.000 & 0.000 & 0.000 & & \\
\hline c3 & 0.523 & 0.171 & 0.025 & 0.114 & 1.117 & 0.371 & -0.253 & 0.027 & 0.000 & 0.000 & 0.000 & 0.000 & 0.000 & 0.000 & 0.000 & \\
\hline c4 & 1.813 & 1.990 & 1.992 & 2.915 & 3.181 & 2.373 & 1.672 & 1.534 & 0.000 & 0.000 & 0.000 & 0.000 & 0.000 & 0.000 & 0.000 & 0.000 \\
\hline
\end{tabular}

Source: own calculations based on NEPS SC3 7.0.0

Note: bold values $p<0.05$ 
Table 21: Standardized residual covariances - revised model - multiple-group path analysis (final) - lower and intermediate track

\begin{tabular}{|c|c|c|c|c|c|c|c|c|c|c|c|c|c|c|c|c|}
\hline & $x 2$ & x3 & $x 4$ & $x 5$ & x6 & $x 7$ & $x 8$ & $\times 9$ & $x 1.2$ & $x 1.3$ & x1.4 & $x 1.5$ & c1 & c2 & c3 & c4 \\
\hline$x 2$ & -3.911 & & & & & & & & & & & & & & & \\
\hline$x 3$ & -3.215 & -1.674 & & & & & & & & & & & & & & \\
\hline$x 4$ & -2.595 & -3.970 & -0.784 & & & & & & & & & & & & & \\
\hline$x 5$ & 2.337 & 2.156 & -0.869 & 3.359 & & & & & & & & & & & & \\
\hline$x 6$ & 0.638 & 2.135 & -0.031 & -4.219 & -5.322 & & & & & & & & & & & \\
\hline$x 7$ & 0.520 & -5.667 & -0.008 & 2.913 & -3.148 & -0.526 & & & & & & & & & & \\
\hline$x 8$ & -0.080 & -3.628 & 0.540 & 3.832 & -3.684 & 0.331 & 0.412 & & & & & & & & & \\
\hline$x 9$ & -0.817 & -5.094 & -2.112 & 1.394 & -0.771 & -0.717 & 0.082 & -0.783 & & & & & & & & \\
\hline $\mathrm{x} 1.2$ & 2.094 & 3.679 & 0.505 & -0.675 & 1.813 & -0.060 & -1.245 & -0.222 & 0.000 & & & & & & & \\
\hline $\mathrm{x} 1.3$ & -1.146 & -0.361 & 1.234 & 0.574 & -0.730 & 0.280 & 0.727 & 0.330 & 0.000 & 0.000 & & & & & & \\
\hline $\mathrm{x} 1.4$ & -1.351 & -1.891 & -1.079 & -0.559 & -2.627 & -0.732 & 0.299 & -0.413 & 0.000 & 0.000 & 0.000 & & & & & \\
\hline $\mathrm{x} 1.5$ & -1.886 & -3.886 & -0.631 & -0.611 & -1.716 & -0.706 & 0.239 & -0.587 & 0.000 & 0.000 & 0.000 & 0.000 & & & & \\
\hline c1 & 0.760 & 0.894 & -0.762 & -0.221 & 0.893 & 0.823 & -0.035 & 0.366 & 0.000 & 0.000 & 0.000 & 0.000 & 0.000 & & & \\
\hline c2 & -1.585 & 1.725 & -0.415 & -3.843 & -1.594 & 0.055 & -0.686 & -0.325 & 0.000 & 0.000 & 0.000 & 0.000 & 0.000 & 0.000 & & \\
\hline c3 & -1.379 & -0.271 & -1.114 & 0.698 & 0.693 & 0.189 & 1.211 & 0.405 & 0.000 & 0.000 & 0.000 & 0.000 & 0.000 & 0.000 & 0.000 & \\
\hline c4 & 2.037 & -3.446 & 3.281 & 0.519 & -0.122 & 0.084 & 0.219 & -0.057 & 0.000 & 0.000 & 0.000 & 0.000 & 0.000 & 0.000 & 0.000 & 0.000 \\
\hline
\end{tabular}

Source: own calculations based on NEPS SC3 7.0.0

Note: bold values $p<0.05$ 
Table 22: Standardized residual covariances - revised model - multiple-group path analysis (final) - higher track

\begin{tabular}{|c|c|c|c|c|c|c|c|c|c|c|c|c|c|c|c|c|}
\hline & $x 2$ & x3 & $x 4$ & $x 5$ & $x 6$ & $x 7$ & $x 8$ & $\times 9$ & $x 1.2$ & $x 1.3$ & x1.4 & $x 1.5$ & c1 & c2 & c3 & c4 \\
\hline $\mathrm{x} 2$ & 2.556 & & & & & & & & & & & & & & & \\
\hline$x 3$ & 4.400 & 3.023 & & & & & & & & & & & & & & \\
\hline$x 4$ & 1.376 & 1.889 & -1.263 & & & & & & & & & & & & & \\
\hline$x 5$ & 2.030 & -0.384 & -0.023 & -4.525 & & & & & & & & & & & & \\
\hline$x 6$ & 0.934 & 3.276 & 3.333 & 5.206 & 6.172 & & & & & & & & & & & \\
\hline$x 7$ & 1.945 & -0.013 & 0.937 & 4.945 & 3.188 & -1.941 & & & & & & & & & & \\
\hline$x 8$ & 0.040 & 1.303 & 0.153 & 4.407 & 3.279 & -2.174 & -2.304 & & & & & & & & & \\
\hline$x 9$ & 1.371 & 1.385 & -0.009 & 2.529 & 1.452 & -1.760 & -1.902 & -1.136 & & & & & & & & \\
\hline $\mathrm{x} 1.2$ & -2.291 & -2.979 & 0.154 & 0.239 & -2.247 & 0.513 & 1.763 & 0.577 & 0.000 & & & & & & & \\
\hline $\mathrm{x} 1.3$ & 0.726 & 0.861 & -0.697 & 0.014 & 0.496 & -0.269 & 0.099 & 0.078 & 0.000 & 0.000 & & & & & & \\
\hline $\mathrm{x} 1.4$ & 0.447 & 2.871 & -1.440 & -0.220 & 3.184 & -0.884 & -1.517 & -0.615 & 0.000 & 0.000 & 0.000 & & & & & \\
\hline $\mathrm{x} 1.5$ & 1.460 & 1.771 & 0.137 & -0.129 & 0.507 & -0.554 & -1.951 & -0.512 & 0.000 & 0.000 & 0.000 & 0.000 & & & & \\
\hline c1 & -0.636 & -1.165 & 0.741 & 0.876 & 0.143 & -0.108 & 0.542 & 0.105 & 0.000 & 0.000 & 0.000 & 0.000 & 0.000 & & & \\
\hline c2 & -0.239 & -1.503 & 0.281 & 4.966 & 1.596 & 0.126 & 2.262 & 0.777 & 0.000 & 0.000 & 0.000 & 0.000 & 0.000 & 0.000 & & \\
\hline c3 & 0.396 & 0.582 & -0.795 & -0.505 & 1.172 & -0.521 & -0.743 & -0.261 & 0.000 & 0.000 & 0.000 & 0.000 & 0.000 & 0.000 & 0.000 & \\
\hline c4 & 1.076 & -0.516 & 2.362 & 1.214 & 2.283 & -1.276 & -0.929 & -0.504 & 0.000 & 0.000 & 0.000 & 0.000 & 0.000 & 0.000 & 0.000 & 0.000 \\
\hline
\end{tabular}

Source: own calculations based on NEPS SC3 7.0.0

Note: bold values $p<0.05$ 
Table 23: Standardized residual covariances - common model - multiple-group path analysis (final) - no tracking

\begin{tabular}{|c|c|c|c|c|c|c|c|c|c|c|c|c|c|}
\hline & $x 2$ & x3 & $x 4$ & x6 & $x 9$ & $x 1.2$ & $x 1.3$ & $\mathrm{x} 1.4$ & $\mathrm{x} 1.5$ & c1 & c2 & c3 & c4 \\
\hline $\mathrm{x2}$ & 1.474 & & & & & & & & & & & & \\
\hline$x 3$ & -0.290 & -0.410 & & & & & & & & & & & \\
\hline$x 4$ & 1.658 & 1.403 & 2.125 & & & & & & & & & & \\
\hline$x 6$ & 1.157 & -0.498 & 0.629 & 0.066 & & & & & & & & & \\
\hline$x 9$ & 2.096 & 1.744 & 2.397 & 2.746 & 1.040 & & & & & & & & \\
\hline $\mathrm{x} 1.2$ & -0.587 & -0.461 & -0.837 & 0.396 & 0.013 & 0.000 & & & & & & & \\
\hline $\mathrm{x} 1.3$ & 0.408 & -0.430 & -0.136 & 0.375 & 0.082 & 0.000 & 0.000 & & & & & & \\
\hline x1.4 & 1.459 & -0.691 & 2.335 & -0.158 & -0.016 & 0.000 & 0.000 & 0.000 & & & & & \\
\hline $\mathrm{x} 1.5$ & 0.630 & 1.635 & 0.533 & 1.381 & 0.326 & 0.000 & 0.000 & 0.000 & 0.000 & & & & \\
\hline c1 & -0.285 & 0.138 & -0.181 & -1.345 & -0.296 & 0.000 & 0.000 & 0.000 & 0.000 & 0.000 & & & \\
\hline c2 & -0.630 & -0.643 & -0.700 & -0.615 & -0.235 & 0.000 & 0.000 & 0.000 & 0.000 & 0.000 & 0.000 & & \\
\hline c3 & 0.524 & 0.170 & 0.024 & 1.113 & 0.259 & 0.000 & 0.000 & 0.000 & 0.000 & 0.000 & 0.000 & 0.000 & \\
\hline c4 & 1.812 & 1.991 & 1.991 & 3.200 & 0.610 & 0.000 & 0.000 & 0.000 & 0.000 & 0.000 & 0.000 & 0.000 & 0.000 \\
\hline
\end{tabular}

Source: own calculations based on NEPS SC3 7.0.0

Note: bold values $p<0.05$ 
Table 24: Standardized residual covariances - common model - multiple-group path analysis (final) - lower and intermediate track

\begin{tabular}{|c|c|c|c|c|c|c|c|c|c|c|c|c|c|}
\hline & $x 2$ & x3 & $x 4$ & x6 & $x 9$ & $x 1.2$ & $x 1.3$ & $\mathrm{x} 1.4$ & $\mathrm{x} 1.5$ & c1 & c2 & c3 & c4 \\
\hline $\mathrm{x2}$ & -3.911 & & & & & & & & & & & & \\
\hline$x 3$ & -3.490 & -3.113 & & & & & & & & & & & \\
\hline$x 4$ & -2.597 & -4.020 & -0.785 & & & & & & & & & & \\
\hline$x 6$ & 0.082 & -0.095 & -2.771 & -0.996 & & & & & & & & & \\
\hline$x 9$ & -0.859 & -4.049 & -1.387 & -0.788 & -0.504 & & & & & & & & \\
\hline $\mathrm{x} 1.2$ & 2.094 & 3.652 & 0.505 & 1.922 & 0.454 & 0.000 & & & & & & & \\
\hline x1.3 & -1.149 & -0.366 & 1.233 & -0.731 & -0.236 & 0.000 & 0.000 & & & & & & \\
\hline x1.4 & -1.351 & -1.854 & -1.081 & -2.661 & -0.512 & 0.000 & 0.000 & 0.000 & & & & & \\
\hline $\mathrm{x} 1.5$ & -1.885 & -3.881 & -0.630 & -1.728 & -0.502 & 0.000 & 0.000 & 0.000 & 0.000 & & & & \\
\hline c1 & 0.757 & 0.886 & -0.762 & 0.942 & 0.176 & 0.000 & 0.000 & 0.000 & 0.000 & 0.000 & & & \\
\hline c2 & -1.589 & 1.704 & -0.411 & -1.389 & -0.339 & 0.000 & 0.000 & 0.000 & 0.000 & 0.000 & 0.000 & & \\
\hline c3 & -1.382 & -0.261 & -1.116 & 0.670 & -0.136 & 0.000 & 0.000 & 0.000 & 0.000 & 0.000 & 0.000 & 0.000 & \\
\hline c4 & 2.037 & -3.441 & 3.284 & -0.105 & 0.140 & 0.000 & 0.000 & 0.000 & 0.000 & 0.000 & 0.000 & 0.000 & 0.000 \\
\hline
\end{tabular}

Source: own calculations based on NEPS SC3 7.0.0

Note: bold values $p<0.05$ 
Table 25: Standardized residual covariances - common model - multiple-group path analysis (final) - higher track

\begin{tabular}{|c|c|c|c|c|c|c|c|c|c|c|c|c|c|}
\hline & $x 2$ & x3 & $x 4$ & x6 & $x 9$ & $x 1.2$ & $x 1.3$ & $\mathrm{x} 1.4$ & $\mathrm{x} 1.5$ & c1 & c2 & c3 & c4 \\
\hline $\mathrm{x2}$ & 2.553 & & & & & & & & & & & & \\
\hline$x 3$ & 4.071 & 3.997 & & & & & & & & & & & \\
\hline$x 4$ & 1.374 & 1.820 & -1.264 & & & & & & & & & & \\
\hline$x 6$ & 0.467 & 0.690 & 1.900 & 0.828 & & & & & & & & & \\
\hline$x 9$ & 1.877 & 1.154 & 0.934 & 2.216 & 0.702 & & & & & & & & \\
\hline $\mathrm{x} 1.2$ & -2.290 & -2.995 & 0.154 & -2.143 & -0.628 & 0.000 & & & & & & & \\
\hline $\mathrm{x} 1.3$ & 0.726 & 0.874 & -0.697 & 0.485 & 0.162 & 0.000 & 0.000 & & & & & & \\
\hline x1.4 & 0.445 & 2.880 & -1.441 & 3.074 & 0.399 & 0.000 & 0.000 & 0.000 & & & & & \\
\hline $\mathrm{x} 1.5$ & 1.458 & 1.776 & 0.135 & 0.482 & 0.241 & 0.000 & 0.000 & 0.000 & 0.000 & & & & \\
\hline c1 & -0.636 & -1.169 & 0.739 & 0.176 & -0.089 & 0.000 & 0.000 & 0.000 & 0.000 & 0.000 & & & \\
\hline c2 & -0.237 & -1.513 & 0.282 & 1.897 & 0.039 & 0.000 & 0.000 & 0.000 & 0.000 & 0.000 & 0.000 & & \\
\hline c3 & 0.398 & 0.581 & -0.795 & 1.160 & 0.233 & 0.000 & 0.000 & 0.000 & 0.000 & 0.000 & 0.000 & 0.000 & \\
\hline c4 & 1.078 & -0.515 & 2.365 & 2.296 & 0.259 & 0.000 & 0.000 & 0.000 & 0.000 & 0.000 & 0.000 & 0.000 & 0.000 \\
\hline
\end{tabular}

Source: own calculations based on NEPS SC3 7.0.0

Note: bold values $p<0.05$ 
Table 26: Results of the path analyses: Common model and revised model

\begin{tabular}{|c|c|c|c|c|c|c|c|c|c|c|}
\hline & \multicolumn{5}{|c|}{$\begin{array}{l}\text { Common model } \\
\text { Chi' }^{2}=46.947 ; \mathrm{df}=91 ; \mathrm{p}\left(\chi^{2}\right)=1.000 ; \mathrm{CFI}=1.000 ; \\
\text { RMSEA }=0.000 ; \operatorname{RMSEA}(\mathrm{Cl} \text { lower bound })=0.000 ; \\
\text { RMSEA }(\mathrm{Cl} \text { upper bound })=0.000 ; \mathrm{SRMR}=0.021\end{array}$} & \multicolumn{5}{|c|}{ 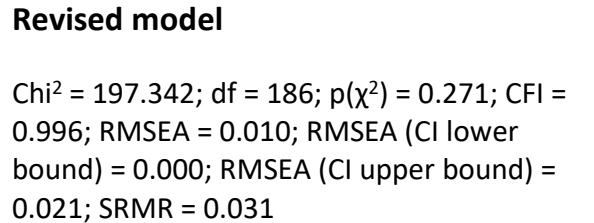 } \\
\hline & \multicolumn{3}{|c|}{ Unstandardized } & \multicolumn{2}{|c|}{ Standardized } & \multicolumn{3}{|c|}{ Unstandardized } & \multicolumn{2}{|c|}{ Standardized } \\
\hline & b & SE & $\mathbf{p}$ & beta & SE & b & SE & p & beta & SE \\
\hline $\begin{array}{l}\text { Parents' high cultural prac- } \\
\text { tices / activities }\end{array}$ & \multicolumn{5}{|c|}{$\begin{array}{l}R^{2} \text { (no tracking) }=0.142 \\
R^{2}(\text { lower and intermed. track })=0.123 \\
R^{2}(\text { higher track })=0.123\end{array}$} & \multicolumn{5}{|c|}{$\begin{array}{l}R^{2} \text { (no tracking) }=0.142 \\
R^{2} \text { (lower and intermed. track) }=0.123 \\
R^{2} \text { (higher track) }=0.123\end{array}$} \\
\hline $\begin{array}{l}\text { Lower secondary education } \\
\text { (Hauptschulabschluss) }\end{array}$ & \multicolumn{3}{|l|}{ Ref. } & & & \multicolumn{2}{|l|}{ Ref. } & & & \\
\hline $\begin{array}{l}\text { Intermediate secondary edu- } \\
\text { cation (Mittlere Reife) }\end{array}$ & 0.584 & 0.098 & 0.000 & 0.226 & 0.038 & 0.584 & 0.098 & 0.000 & 0.226 & 0.038 \\
\hline $\begin{array}{l}\text { Higher secondary education } \\
\text { (Abitur) }\end{array}$ & 1.189 & 0.120 & 0.000 & 0.459 & 0.046 & 1.189 & 0.120 & 0.000 & 0.459 & 0.046 \\
\hline $\begin{array}{l}\text { Lower tertiary education } \\
\text { (Fachochschulabschluss) }\end{array}$ & 1.863 & 0.146 & 0.000 & 0.719 & 0.056 & 1.864 & 0.146 & 0.000 & 0.719 & 0.056 \\
\hline $\begin{array}{l}\text { Higher tertiary education } \\
\text { (Hochschulabschluss) }\end{array}$ & 2.771 & 0.142 & 0.000 & 1.070 & 0.055 & 2.772 & 0.142 & 0.000 & 1.070 & 0.055 \\
\hline Migration background & -0.186 & 0.076 & 0.014 & -0.072 & 0.029 & -0.186 & 0.076 & 0.014 & -0.072 & 0.029 \\
\hline Intercept (no tracking) & 4.971 & 0.163 & 0.000 & & & 4.971 & 0.163 & 0.000 & & \\
\hline $\begin{array}{l}\text { Intercept (lower and interme- } \\
\text { diate track) }\end{array}$ & 4.434 & 0.101 & 0.000 & & & 4.435 & 0.101 & 0.000 & & \\
\hline Intercept (higher track) & 5.319 & 0.139 & 0.000 & & & 5.319 & 0.139 & 0.000 & & \\
\hline $\begin{array}{l}\text { Students' high cultural prac- } \\
\text { tices / activities }\end{array}$ & \multicolumn{5}{|c|}{$\begin{array}{l}R^{2} \text { (no tracking) }=0.070 \\
R^{2} \text { (lower and intermed. track) }=0.049 \\
R^{2} \text { (higher track) }=0.088\end{array}$} & \multicolumn{5}{|c|}{$\begin{array}{l}R^{2} \text { (no tracking) }=0.068 \\
\left.R^{2} \text { (lower and intermed. track }\right)=0.048 \\
R^{2}(\text { higher track })=0.085\end{array}$} \\
\hline $\begin{array}{l}\text { Lower secondary education } \\
\text { (Hauptschulabschluss) }\end{array}$ & Ref. & & & & & Ref. & & & & \\
\hline cation (Mittlere Reife) & -0.175 & 0.127 & 0.169 & -0.071 & 0.051 & -0.172 & 0.127 & 0.176 & -0.070 & 0.052 \\
\hline $\begin{array}{l}\text { Higher secondary education } \\
\text { (Abitur) }\end{array}$ & -0.269 & 0.136 & 0.049 & -0.109 & 0.055 & -0.262 & 0.136 & 0.055 & -0.106 & 0.055 \\
\hline $\begin{array}{l}\text { Lower tertiary education } \\
\text { (Fachochschulabschluss) }\end{array}$ & -0.067 & 0.173 & 0.696 & -0.027 & 0.070 & -0.059 & 0.173 & 0.735 & -0.024 & 0.070 \\
\hline
\end{tabular}




\begin{tabular}{|c|c|c|c|c|c|c|c|c|c|c|}
\hline & \multicolumn{5}{|c|}{ Common model } & \multicolumn{5}{|c|}{ Revised model } \\
\hline & \multicolumn{3}{|c|}{ Unstandardized } & \multicolumn{2}{|c|}{ Standardized } & \multicolumn{3}{|c|}{ Unstandardized } & \multicolumn{2}{|c|}{ Standardized } \\
\hline & $\mathbf{b}$ & SE & $\mathbf{p}$ & beta & SE & b & SE & $\mathbf{p}$ & beta & SE \\
\hline $\begin{array}{l}\text { Higher tertiary education } \\
\text { (Hochschulabschluss) }\end{array}$ & 0.185 & 0.158 & 0.241 & 0.075 & 0.064 & 0.200 & 0.159 & 0.208 & 0.081 & 0.064 \\
\hline Migration background & 0.166 & 0.078 & 0.033 & 0.067 & 0.032 & 0.165 & 0.078 & 0.035 & 0.067 & 0.032 \\
\hline Male & -0.313 & 0.072 & 0.000 & -0.127 & 0.029 & -0.314 & 0.072 & 0.000 & -0.127 & 0.029 \\
\hline $\begin{array}{l}\text { Parents' high cultural prac- } \\
\text { tices / activities }\end{array}$ & 0.233 & 0.017 & 0.000 & 0.244 & 0.017 & 0.227 & 0.016 & 0.000 & 0.238 & 0.017 \\
\hline Intercept (no tracking) & 5.066 & 0.201 & 0.000 & & & 5.094 & 0.200 & 0.000 & & \\
\hline $\begin{array}{l}\text { Intercept (lower and interme- } \\
\text { diate track) }\end{array}$ & 4.784 & 0.154 & 0.000 & & & 4.809 & 0.153 & 0.000 & & \\
\hline Intercept (higher track) & 5.003 & 0.172 & 0.000 & & & 5.033 & 0.171 & 0.000 & & \\
\hline $\begin{array}{l}\text { Number of books in the } \\
\text { home }\end{array}$ & \multicolumn{5}{|c|}{$\begin{array}{l}R^{2} \text { (no tracking) }=0.300 \\
\left.R^{2} \text { (lower and intermed. track }\right)=0.206 \\
R^{2} \text { (higher track) }=0.266\end{array}$} & \multicolumn{5}{|c|}{$\begin{array}{l}R^{2} \text { (no tracking) }=0.300 \\
\left.R^{2} \text { (lower and intermed. track }\right)=0.206 \\
R^{2}(\text { higher track })=0.266\end{array}$} \\
\hline $\begin{array}{l}\text { Lower secondary education } \\
\text { (Hauptschulabschluss) }\end{array}$ & Ref. & & & & & Ref. & & & & \\
\hline $\begin{array}{l}\text { Intermediate secondary edu- } \\
\text { cation (Mittlere Reife) }\end{array}$ & 0.569 & 0.054 & 0.000 & 0.441 & 0.042 & 0.569 & 0.054 & 0.000 & 0.441 & 0.042 \\
\hline $\begin{array}{l}\text { Higher secondary education } \\
\text { (Abitur) }\end{array}$ & 1.077 & 0.059 & 0.000 & 0.834 & 0.046 & 1.077 & 0.059 & 0.000 & 0.834 & 0.046 \\
\hline $\begin{array}{l}\text { Lower tertiary education } \\
\text { (Fachochschulabschluss) }\end{array}$ & 1.441 & 0.073 & 0.000 & 1.116 & 0.057 & 1.441 & 0.073 & 0.000 & 1.116 & 0.057 \\
\hline $\begin{array}{l}\text { Higher tertiary education } \\
\text { (Hochschulabschluss) }\end{array}$ & 1.943 & 0.063 & 0.000 & 1.505 & 0.049 & 1.943 & 0.063 & 0.000 & 1.505 & 0.049 \\
\hline Migration background & -0.275 & 0.039 & 0.000 & -0.213 & 0.030 & -0.275 & 0.039 & 0.000 & -0.213 & 0.030 \\
\hline Intercept (no tracking) & 3.138 & 0.080 & 0.000 & & & 3.138 & 0.080 & 0.000 & & \\
\hline $\begin{array}{l}\text { Intercept (lower and interme- } \\
\text { diate track) }\end{array}$ & 2.997 & 0.058 & 0.000 & & & 2.997 & 0.058 & 0.000 & & \\
\hline Intercept (higher track) & 3.310 & 0.062 & 0.000 & & & 3.310 & 0.062 & 0.000 & & \\
\hline Attitudes towards reading & & & & & & \multicolumn{5}{|c|}{$\begin{array}{l}R^{2} \text { (no tracking) }=0.064 \\
R^{2} \text { (lower and intermed. track) }=0.055 \\
R^{2} \text { (higher track) }=0.083\end{array}$} \\
\hline $\begin{array}{l}\text { Lower secondary education } \\
\text { (Hauptschulabschluss) }\end{array}$ & & & & & & Ref. & & & & \\
\hline
\end{tabular}




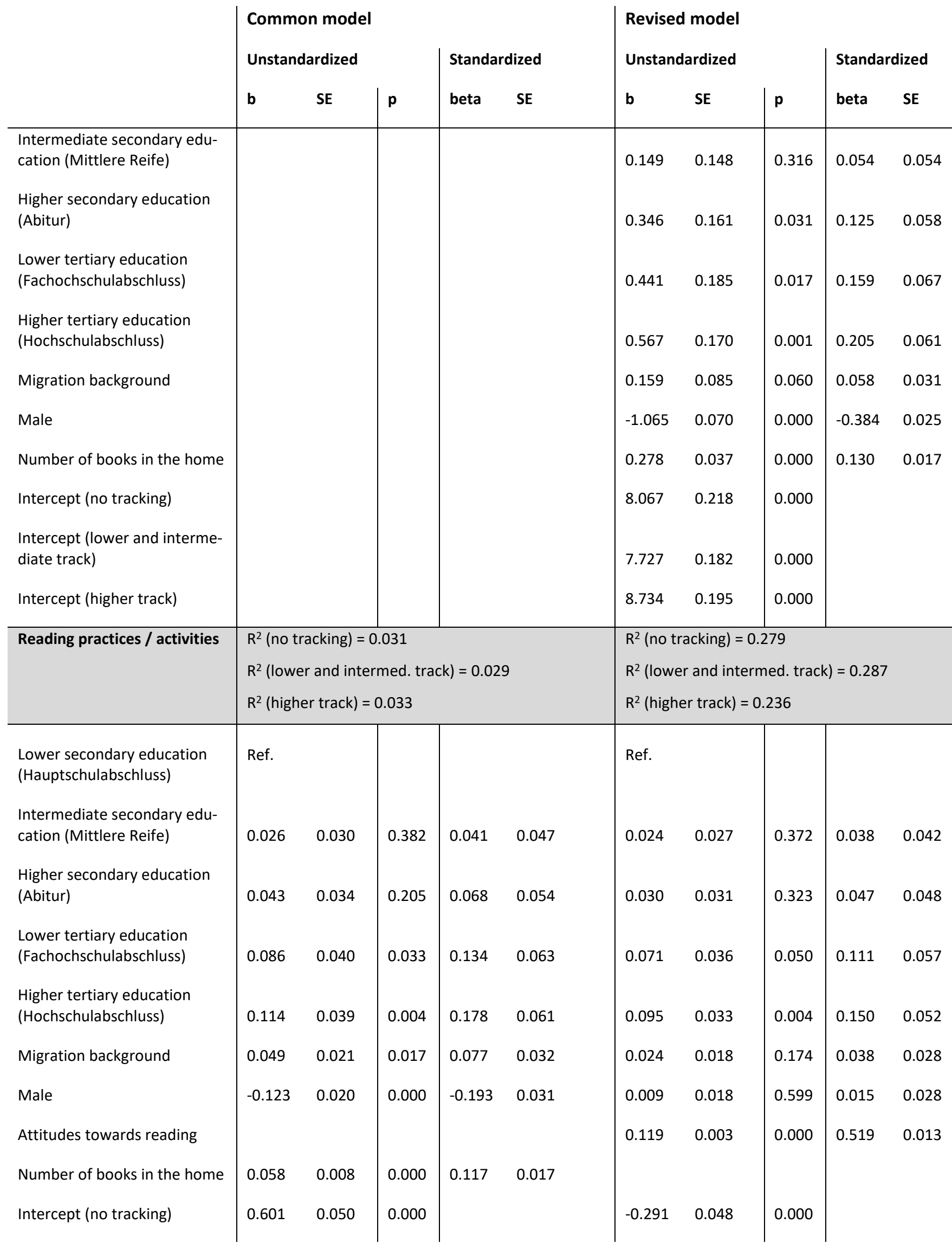




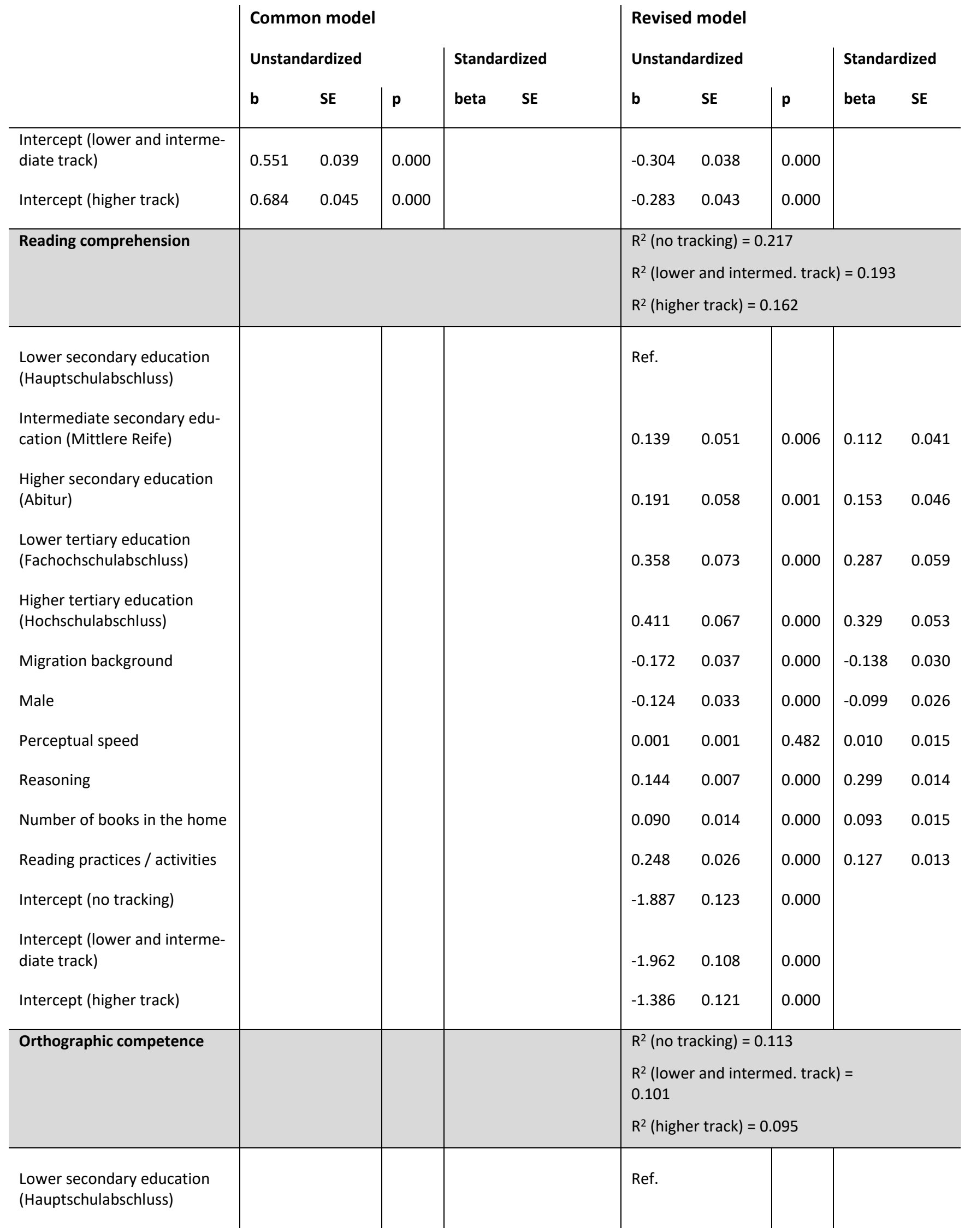




\begin{tabular}{|c|c|c|c|c|c|c|c|c|c|c|}
\hline & \multicolumn{5}{|c|}{ Common model } & \multicolumn{5}{|c|}{ Revised model } \\
\hline & \multicolumn{3}{|c|}{ Unstandardized } & \multicolumn{2}{|c|}{ Standardized } & \multicolumn{3}{|c|}{ Unstandardized } & \multicolumn{2}{|c|}{ Standardized } \\
\hline & b & SE & $\mathbf{p}$ & beta & SE & b & SE & $\mathbf{p}$ & beta & SE \\
\hline $\begin{array}{l}\text { Intermediate secondary edu- } \\
\text { cation (Mittlere Reife) }\end{array}$ & & & & & & 0.253 & 0.063 & 0.000 & 0.187 & 0.047 \\
\hline $\begin{array}{l}\text { Higher secondary education } \\
\text { (Abitur) }\end{array}$ & & & & & & 0.339 & 0.072 & 0.000 & 0.250 & 0.053 \\
\hline $\begin{array}{l}\text { Lower tertiary education } \\
\text { (Fachochschulabschluss) }\end{array}$ & & & & & & 0.323 & 0.089 & 0.000 & 0.238 & 0.066 \\
\hline $\begin{array}{l}\text { Higher tertiary education } \\
\text { (Hochschulabschluss) }\end{array}$ & & & & & & 0.429 & 0.078 & 0.000 & 0.317 & 0.058 \\
\hline Migration background & & & & & & -0.115 & 0.042 & 0.006 & -0.085 & 0.031 \\
\hline Male & & & & & & -0.335 & 0.034 & 0.000 & -0.247 & 0.025 \\
\hline Perceptual speed & & & & & & 0.002 & 0.002 & 0.249 & 0.019 & 0.016 \\
\hline Reasoning & & & & & & 0.099 & 0.009 & 0.000 & 0.190 & 0.016 \\
\hline Number of books in the home & & & & & & 0.031 & 0.016 & 0.045 & 0.030 & 0.015 \\
\hline Reading practices / activities & & & & & & 0.209 & 0.028 & 0.000 & 0.099 & 0.013 \\
\hline Intercept (no tracking) & & & & & & -1.498 & 0.155 & 0.000 & & \\
\hline $\begin{array}{l}\text { Intercept (lower and interme- } \\
\text { diate track) }\end{array}$ & & & & & & -1.528 & 0.142 & 0.000 & & \\
\hline Intercept (higher track) & & & & & & -0.633 & 0.155 & 0.000 & & \\
\hline $\begin{array}{l}\text { Average grade in German } \\
\text { and Mathematics } \\
\text { (no tracking) }\end{array}$ & $\mathrm{R}^{2}=0.1$ & & & & & $\mathrm{R}^{2}=0.2$ & & & & \\
\hline $\begin{array}{l}\text { Lower secondary education } \\
\text { (Hauptschulabschluss) }\end{array}$ & Ref. & & & & & Ref. & & & & \\
\hline $\begin{array}{l}\text { Intermediate secondary edu- } \\
\text { cation (Mittlere Reife) }\end{array}$ & 0.029 & 0.106 & 0.782 & 0.030 & 0.108 & -0.100 & 0.111 & 0.368 & -0.102 & 0.113 \\
\hline $\begin{array}{l}\text { Higher secondary education } \\
\text { (Abitur) }\end{array}$ & 0.146 & 0.106 & 0.167 & 0.149 & 0.108 & 0.011 & 0.101 & 0.916 & 0.011 & 0.103 \\
\hline $\begin{array}{l}\text { Lower tertiary education } \\
\text { (Fachochschulabschluss) }\end{array}$ & 0.460 & 0.175 & 0.008 & 0.469 & 0.178 & 0.215 & 0.153 & 0.160 & 0.219 & 0.156 \\
\hline $\begin{array}{l}\text { Higher tertiary education } \\
\text { (Hochschulabschluss) }\end{array}$ & 0.282 & 0.132 & 0.033 & 0.288 & 0.135 & 0.035 & 0.131 & 0.792 & 0.035 & 0.134 \\
\hline Migration background & 0.013 & 0.077 & 0.864 & 0.013 & 0.078 & 0.075 & 0.068 & 0.274 & 0.076 & 0.070 \\
\hline Male & -0.125 & 0.065 & 0.057 & -0.127 & 0.067 & 0.004 & 0.060 & 0.950 & 0.004 & 0.061 \\
\hline
\end{tabular}




\begin{tabular}{|c|c|c|c|c|c|c|c|c|c|c|}
\hline & \multicolumn{5}{|c|}{ Common model } & \multicolumn{5}{|c|}{ Revised model } \\
\hline & \multicolumn{3}{|c|}{ Unstandardized } & \multicolumn{2}{|c|}{ Standardized } & \multicolumn{3}{|c|}{ Unstandardized } & \multicolumn{2}{|c|}{ Standardized } \\
\hline & b & SE & $\mathbf{p}$ & beta & SE & b & SE & $\mathbf{p}$ & beta & SE \\
\hline Perceptual speed & 0.001 & 0.003 & 0.780 & 0.012 & 0.043 & 0.001 & 0.003 & 0.686 & 0.014 & 0.035 \\
\hline Reasoning & 0.110 & 0.014 & 0.000 & 0.293 & 0.036 & 0.052 & 0.014 & 0.000 & 0.138 & 0.037 \\
\hline $\begin{array}{l}\text { Students' high cultural prac- } \\
\text { tices / activities }\end{array}$ & 0.016 & 0.018 & 0.396 & 0.040 & 0.047 & 0.017 & 0.016 & 0.296 & 0.043 & 0.041 \\
\hline $\begin{array}{l}\text { Parents' high cultural prac- } \\
\text { tices / activities }\end{array}$ & 0.010 & 0.013 & 0.452 & 0.027 & 0.036 & -0.003 & 0.014 & 0.842 & -0.007 & 0.037 \\
\hline Reading comprehension & & & & & & 0.164 & 0.035 & 0.000 & 0.209 & 0.045 \\
\hline Orthographic competence & & & & & & 0.224 & 0.033 & 0.000 & 0.310 & 0.045 \\
\hline Reading activities & 0.176 & 0.056 & 0.002 & 0.114 & 0.036 & & & & & \\
\hline Intercept & -1.147 & 0.222 & 0.000 & & & -0.360 & 0.224 & 0.108 & & \\
\hline $\begin{array}{l}\text { Average grade in German } \\
\text { and Mathematics } \\
\text { (lower and intermediate } \\
\text { track) }\end{array}$ & \multicolumn{5}{|c|}{$R^{2}=0.050$} & \multicolumn{5}{|c|}{$R^{2}=0.125$} \\
\hline $\begin{array}{l}\text { Lower secondary education } \\
\text { (Hauptschulabschluss) }\end{array}$ & Ref. & & & & & Ref. & & & & \\
\hline $\begin{array}{l}\text { Intermediate secondary edu- } \\
\text { cation (Mittlere Reife) }\end{array}$ & 0.016 & 0.062 & 0.799 & 0.016 & 0.064 & -0.038 & 0.062 & 0.533 & -0.039 & 0.063 \\
\hline $\begin{array}{l}\text { Higher secondary education } \\
\text { (Abitur) }\end{array}$ & 0.092 & 0.083 & 0.264 & 0.094 & 0.084 & -0.002 & 0.078 & 0.983 & -0.002 & 0.080 \\
\hline $\begin{array}{l}\text { Lower tertiary education } \\
\text { (Fachochschulabschluss) }\end{array}$ & 0.129 & 0.111 & 0.244 & 0.132 & 0.113 & 0.015 & 0.110 & 0.893 & 0.015 & 0.113 \\
\hline $\begin{array}{l}\text { Higher tertiary education } \\
\text { (Hochschulabschluss) }\end{array}$ & 0.250 & 0.115 & 0.030 & 0.255 & 0.117 & 0.107 & 0.106 & 0.313 & 0.109 & 0.108 \\
\hline Migration background & -0.196 & 0.055 & 0.000 & -0.199 & 0.056 & -0.157 & 0.055 & 0.004 & -0.160 & 0.056 \\
\hline Male & -0.016 & 0.051 & 0.756 & -0.016 & 0.052 & 0.059 & 0.049 & 0.233 & 0.060 & 0.050 \\
\hline Perceptual speed & 0.007 & 0.002 & 0.001 & 0.090 & 0.027 & 0.006 & 0.002 & 0.004 & 0.078 & 0.027 \\
\hline Reasoning & 0.049 & 0.010 & 0.000 & 0.130 & 0.027 & 0.016 & 0.010 & 0.097 & 0.043 & 0.026 \\
\hline $\begin{array}{l}\text { Students' high cultural prac- } \\
\text { tices / activities }\end{array}$ & 0.006 & 0.010 & 0.552 & 0.015 & 0.025 & 0.018 & 0.010 & 0.060 & 0.045 & 0.024 \\
\hline $\begin{array}{l}\text { Parents' high cultural prac- } \\
\text { tices / activities }\end{array}$ & 0.023 & 0.012 & 0.045 & 0.062 & 0.031 & 0.019 & 0.011 & 0.086 & 0.050 & 0.029 \\
\hline Reading comprehension & & & & & & 0.121 & 0.025 & 0.000 & 0.154 & 0.032 \\
\hline
\end{tabular}




\begin{tabular}{|c|c|c|c|c|c|c|c|c|c|c|}
\hline & \multicolumn{5}{|c|}{ Common model } & \multicolumn{5}{|c|}{ Revised model } \\
\hline & \multicolumn{3}{|c|}{ Unstandardized } & \multicolumn{2}{|c|}{ Standardized } & \multicolumn{3}{|c|}{ Unstandardized } & \multicolumn{2}{|c|}{ Standardized } \\
\hline & b & SE & $\mathbf{p}$ & beta & SE & b & SE & $\mathbf{p}$ & beta & SE \\
\hline Orthographic competence & & & & & & 0.164 & 0.024 & 0.000 & 0.227 & 0.034 \\
\hline Reading activities & 0.049 & 0.038 & 0.205 & 0.032 & 0.025 & & & & & \\
\hline Intercept & -0.748 & 0.140 & 0.000 & & & -0.362 & 0.139 & 0.009 & & \\
\hline $\begin{array}{l}\text { Average grade in German } \\
\text { and Mathematics< } \\
\text { (Higher track) }\end{array}$ & \multicolumn{5}{|c|}{$R^{2}=0.118$} & \multicolumn{5}{|c|}{$R^{2}=0.241$} \\
\hline $\begin{array}{l}\text { Lower secondary education } \\
\text { (Hauptschulabschluss) }\end{array}$ & Ref. & & & & & Ref. & & & & \\
\hline $\begin{array}{l}\text { Intermediate secondary edu- } \\
\text { cation (Mittlere Reife) }\end{array}$ & 0.140 & 0.119 & 0.240 & 0.142 & 0.121 & 0.086 & 0.115 & 0.455 & 0.088 & 0.117 \\
\hline $\begin{array}{l}\text { Higher secondary education } \\
\text { (Abitur) }\end{array}$ & 0.262 & 0.113 & 0.020 & 0.267 & 0.115 & 0.192 & 0.109 & 0.078 & 0.196 & 0.111 \\
\hline $\begin{array}{l}\text { Lower tertiary education } \\
\text { (Fachochschulabschluss) }\end{array}$ & 0.229 & 0.125 & 0.067 & 0.234 & 0.127 & 0.163 & 0.124 & 0.189 & 0.166 & 0.126 \\
\hline $\begin{array}{l}\text { Higher tertiary education } \\
\text { (Hochschulabschluss) }\end{array}$ & 0.422 & 0.117 & 0.000 & 0.430 & 0.120 & 0.309 & 0.118 & 0.009 & 0.316 & 0.121 \\
\hline Migration background & -0.199 & 0.046 & 0.000 & -0.203 & 0.047 & -0.147 & 0.042 & 0.000 & -0.150 & 0.043 \\
\hline Male & -0.110 & 0.049 & 0.024 & -0.112 & 0.050 & -0.028 & 0.045 & 0.528 & -0.029 & 0.045 \\
\hline Perceptual speed & 0.006 & 0.002 & 0.001 & 0.080 & 0.024 & 0.006 & 0.002 & 0.000 & 0.078 & 0.022 \\
\hline Reasoning & 0.101 & 0.010 & 0.000 & 0.268 & 0.027 & 0.066 & 0.010 & 0.000 & 0.174 & 0.026 \\
\hline $\begin{array}{l}\text { Students' high cultural prac- } \\
\text { tices / activities }\end{array}$ & 0.016 & 0.008 & 0.036 & 0.041 & 0.020 & 0.015 & 0.007 & 0.044 & 0.037 & 0.019 \\
\hline $\begin{array}{l}\text { Parents' high cultural prac- } \\
\text { tices / activities }\end{array}$ & 0.018 & 0.009 & 0.050 & 0.048 & 0.024 & 0.015 & 0.009 & 0.089 & 0.039 & 0.023 \\
\hline Reading comprehension & & & & & & 0.099 & 0.024 & 0.000 & 0.126 & 0.030 \\
\hline Orthographic competence & & & & & & 0.263 & 0.020 & 0.000 & 0.363 & 0.028 \\
\hline Reading activities & 0.103 & 0.035 & 0.003 & 0.067 & 0.023 & & & & & \\
\hline Intercept & -1.539 & 0.164 & 0.000 & & & -1.343 & 0.168 & 0.000 & & \\
\hline
\end{tabular}

Source: own calculations based on NEPS SC3 7.0.0

Notes: $n=4,924 ; b$ is the unstandardized regression coefficients; beta is the standardized regression coefficient: If $y$ and $x$ are continuous, beta is standardized according to $\mathrm{SD}_{\mathrm{y}}$ and $\mathrm{SD}_{\mathrm{x}}$. If $\mathrm{y}$ is continuous and $\mathrm{x}$ binary, beta is standardized according to $\mathrm{SD}_{\mathrm{y}}$. 
Table 27: Direct, indirect, and total effects of parental education on mediating variables - common model

\begin{tabular}{|c|c|c|c|c|c|c|c|}
\hline \multirow{2}{*}{$\begin{array}{l} \\
\begin{array}{l}\text { Students' high cultural participa- } \\
\text { tion }\end{array}\end{array}$} & & via & \multicolumn{3}{|c|}{ Unstandardized } & $\begin{array}{l}\text { Standa } \\
\text { beta }\end{array}$ & $\begin{array}{l}\text { zed } \\
\text { SE }\end{array}$ \\
\hline & & & & & & & \\
\hline Intermediate secondary education & $\begin{array}{l}\text { Direct effect } \\
\text { Indirect effect } \\
\text { Total effect }\end{array}$ & $\rightarrow$ Parents' high cultural participation & $\begin{array}{l}-0.177 \\
0.136 \\
-0.041\end{array}$ & $\begin{array}{l}0.127 \\
0.025 \\
0.129\end{array}$ & $\begin{array}{l}0.163 \\
0.000 \\
0.751\end{array}$ & $\begin{array}{l}-0.072 \\
0.055 \\
-0.017\end{array}$ & $\begin{array}{l}0.051 \\
0.010 \\
0.052\end{array}$ \\
\hline Higher secondary education & $\begin{array}{l}\text { Direct effect } \\
\text { Indirect effect } \\
\text { Total effect }\end{array}$ & $\rightarrow$ Parents' high cultural participation & $\begin{array}{l}-0.269 \\
0.277 \\
0.007\end{array}$ & $\begin{array}{l}0.136 \\
0.033 \\
0.138\end{array}$ & $\begin{array}{l}0.047 \\
0.000 \\
0.957\end{array}$ & $\begin{array}{l}-0.109 \\
0.112 \\
0.003\end{array}$ & $\begin{array}{l}0.055 \\
0.013 \\
0.056\end{array}$ \\
\hline Lower tertiary education & $\begin{array}{l}\text { Direct effect } \\
\text { Indirect effect } \\
\text { Total effect }\end{array}$ & $\rightarrow$ Parents' high cultural participation & $\begin{array}{l}-0.069 \\
0.434 \\
0.365\end{array}$ & $\begin{array}{l}0.172 \\
0.047 \\
0.171\end{array}$ & $\begin{array}{l}0.688 \\
0.000 \\
0.033\end{array}$ & $\begin{array}{l}-0.028 \\
0.176 \\
0.148\end{array}$ & $\begin{array}{l}0.070 \\
0.019 \\
0.069\end{array}$ \\
\hline Higher tertiary education & $\begin{array}{l}\text { Direct effect } \\
\text { Indirect effect } \\
\text { Total effect }\end{array}$ & $\rightarrow$ Parents' high cultural participation & $\begin{array}{l}0.183 \\
0.646 \\
0.829\end{array}$ & $\begin{array}{l}0.158 \\
0.055 \\
0.161\end{array}$ & $\begin{array}{l}0.245 \\
0.000 \\
0.000\end{array}$ & $\begin{array}{l}0.074 \\
0.261 \\
0.335\end{array}$ & $\begin{array}{l}0.064 \\
0.022 \\
0.065\end{array}$ \\
\hline \multicolumn{8}{|l|}{ Reading activities } \\
\hline Intermediate secondary education & $\begin{array}{l}\text { Direct effect } \\
\text { Indirect effect }\end{array}$ & $\rightarrow$ Number of books in the home & $\begin{array}{l}0.026 \\
0.033\end{array}$ & $\begin{array}{l}0.030 \\
0.005\end{array}$ & $\begin{array}{l}0.382 \\
0.000\end{array}$ & $\begin{array}{l}0.041 \\
0.052\end{array}$ & $\begin{array}{l}0.046 \\
0.009\end{array}$ \\
\hline
\end{tabular}




\begin{tabular}{|c|c|c|c|c|c|c|c|}
\hline & & via & Unsta & rdized & & Stand & ized \\
\hline & & & b & SE & $\mathbf{p}$ & beta & SE \\
\hline & Total effect & & 0.059 & 0.029 & 0.045 & 0.092 & 0.046 \\
\hline Higher secondary education & Direct effect & & 0.043 & 0.034 & 0.204 & 0.068 & 0.053 \\
\hline & Indirect effect & $\rightarrow$ Number of books in the home & 0.062 & 0.010 & 0.000 & 0.097 & 0.015 \\
\hline & Total effect & & 0.105 & 0.033 & 0.001 & 0.165 & 0.052 \\
\hline Lower tertiary education & Direct effect & & 0.086 & 0.040 & 0.033 & 0.134 & 0.063 \\
\hline & Indirect effect & $\rightarrow$ Number of books in the home & 0.083 & 0.013 & 0.000 & 0.130 & 0.020 \\
\hline & Total effect & & 0.169 & 0.038 & 0.000 & 0.264 & 0.060 \\
\hline Higher tertiary education & Direct effect & & 0.113 & 0.039 & 0.003 & 0.178 & 0.061 \\
\hline & Indirect effect & $\rightarrow$ Number of books in the home & 0.112 & 0.017 & 0.000 & 0.176 & 0.026 \\
\hline & Total effect & & 0.226 & 0.036 & 0.000 & 0.354 & 0.057 \\
\hline
\end{tabular}

Source: own calculations based on NEPS SC3 7.0 .0

Notes: $n=4,924$; lower secondary education is the reference category; $b$ is the unstandardized regression coefficients; beta is the standardized regression coefficient: beta is standardized according to $\mathrm{SD}_{\mathrm{y}}$. 
Table 28: Direct, indirect, and total effects of parental education on students' grades - common model

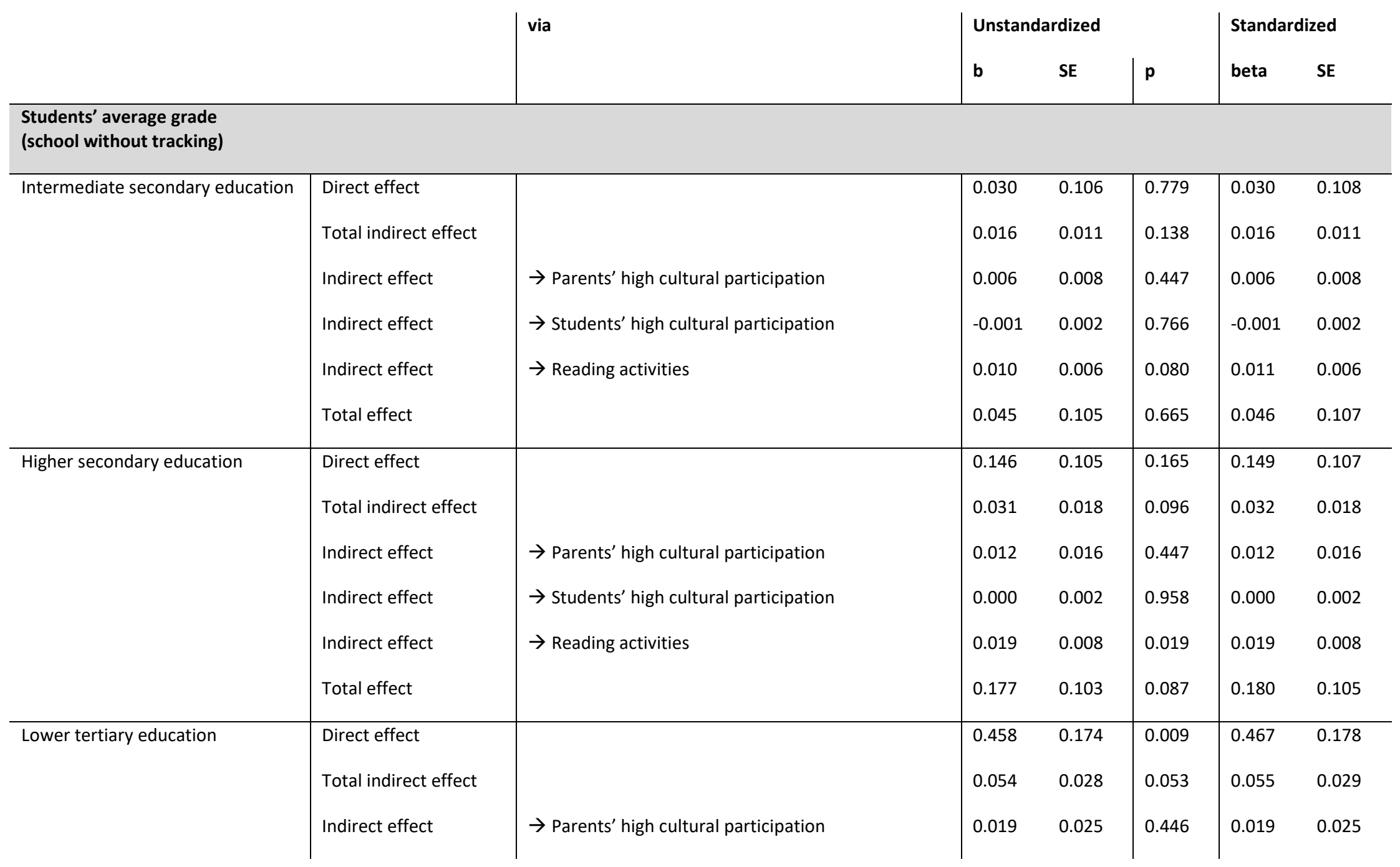









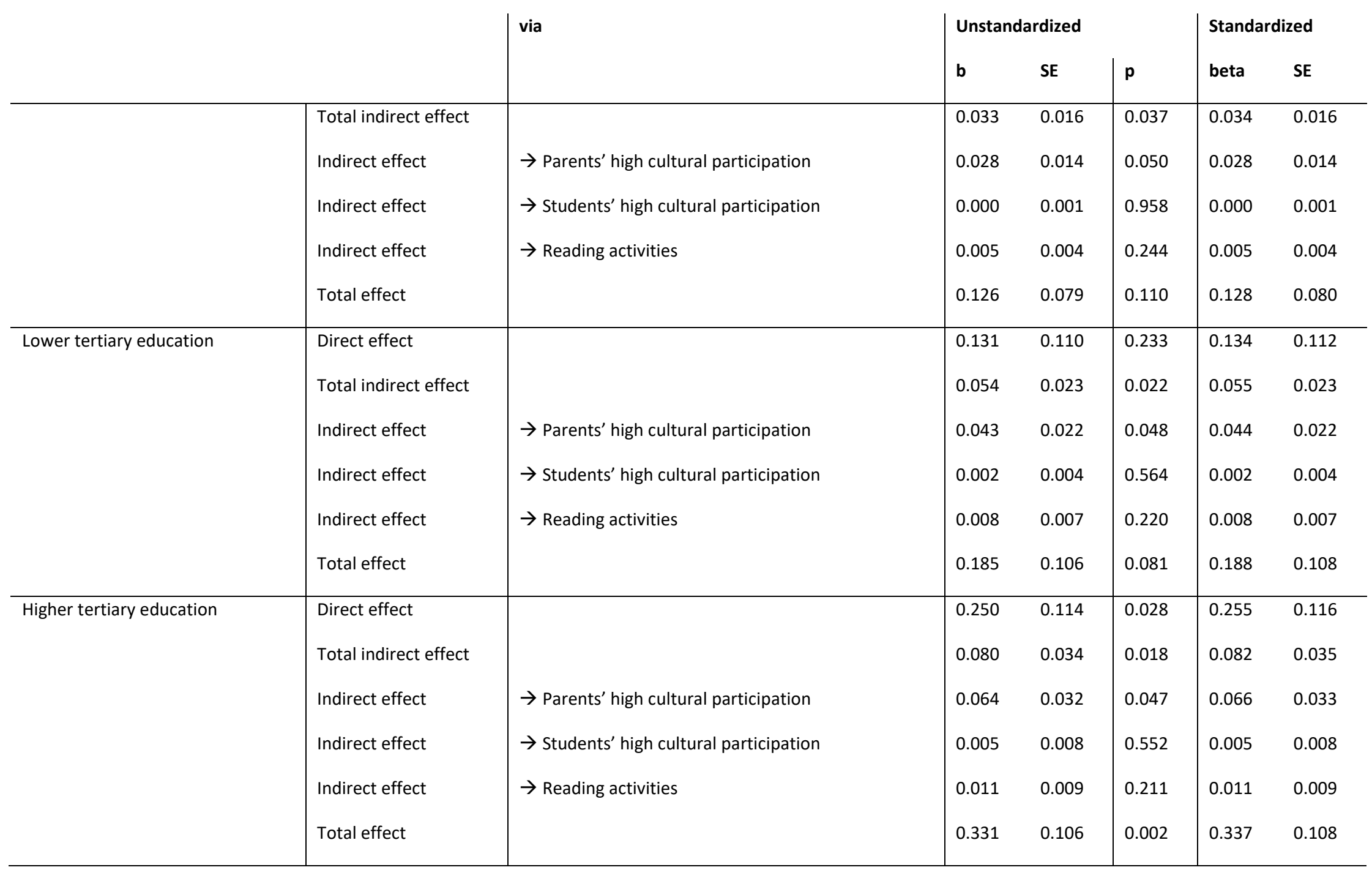




\begin{tabular}{|c|c|c|c|c|c|c|c|}
\hline \multirow{3}{*}{$\begin{array}{l}\text { Students' average grade } \\
\text { (higher track) }\end{array}$} & & \multirow[t]{2}{*}{ via } & \multicolumn{3}{|c|}{ Unstandardized } & \multicolumn{2}{|c|}{ Standardized } \\
\hline & & & b & SE & $\mathbf{p}$ & beta & SE \\
\hline & & & & & & & \\
\hline Intermediate secondary education & $\begin{array}{l}\text { Direct effect } \\
\text { Total indirect effect } \\
\text { Indirect effect } \\
\text { Indirect effect } \\
\text { Indirect effect } \\
\text { Total effect }\end{array}$ & $\begin{array}{l}\rightarrow \text { Parents' high cultural participation } \\
\rightarrow \text { Students' high cultural participation } \\
\rightarrow \text { Reading activities }\end{array}$ & $\begin{array}{l}0.141 \\
0.016 \\
0.010 \\
-0.001 \\
0.006 \\
0.157\end{array}$ & $\begin{array}{l}0.119 \\
0.007 \\
0.006 \\
0.002 \\
0.004 \\
0.118\end{array}$ & $\begin{array}{l}0.235 \\
0.034 \\
0.070 \\
0.754 \\
0.096 \\
0.182\end{array}$ & $\begin{array}{l}0.144 \\
0.016 \\
0.011 \\
-0.001 \\
0.006 \\
0.160\end{array}$ & $\begin{array}{l}0.121 \\
0.007 \\
0.006 \\
0.002 \\
0.004 \\
0.120\end{array}$ \\
\hline Higher secondary education & $\begin{array}{l}\text { Direct effect } \\
\text { Total indirect effect } \\
\text { Indirect effect } \\
\text { Indirect effect } \\
\text { Indirect effect } \\
\text { Total effect }\end{array}$ & $\begin{array}{l}\rightarrow \text { Parents' high cultural participation } \\
\rightarrow \text { Students' high cultural participation } \\
\rightarrow \text { Reading activities }\end{array}$ & $\begin{array}{l}0.263 \\
0.032 \\
0.021 \\
0.000 \\
0.011 \\
0.296\end{array}$ & $\begin{array}{l}0.113 \\
0.012 \\
0.011 \\
0.002 \\
0.005 \\
0.110\end{array}$ & $\begin{array}{l}0.020 \\
0.007 \\
0.055 \\
0.957 \\
0.030 \\
0.007\end{array}$ & $\begin{array}{l}0.269 \\
0.033 \\
0.022 \\
0.000 \\
0.011 \\
0.302\end{array}$ & $\begin{array}{l}0.115 \\
0.012 \\
0.011 \\
0.002 \\
0.005 \\
0.113\end{array}$ \\
\hline Lower tertiary education & $\begin{array}{l}\text { Direct effect } \\
\text { Total indirect effect } \\
\text { Indirect effect } \\
\text { Indirect effect }\end{array}$ & $\begin{array}{l}\rightarrow \text { Parents' high cultural participation } \\
\rightarrow \text { Students' high cultural participation }\end{array}$ & $\begin{array}{l}0.231 \\
0.057 \\
0.033 \\
0.006\end{array}$ & $\begin{array}{l}0.125 \\
0.019 \\
0.018 \\
0.004\end{array}$ & $\begin{array}{l}0.064 \\
0.002 \\
0.057 \\
0.113\end{array}$ & $\begin{array}{l}0.236 \\
0.058 \\
0.034 \\
0.006\end{array}$ & $\begin{array}{l}0.127 \\
0.019 \\
0.018 \\
0.004\end{array}$ \\
\hline
\end{tabular}




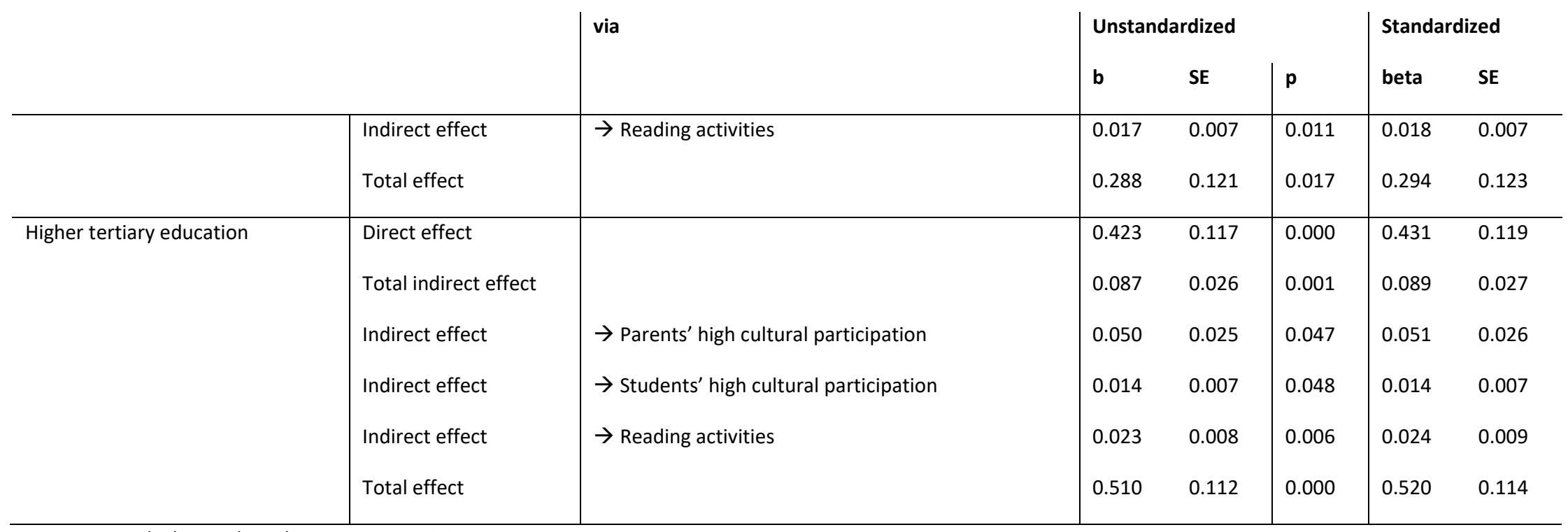

Source: own calculations based on NEPS SC3 7.0 .0

Notes: $n=4,924$; lower secondary education is the reference category; $b$ is the unstandardized regression coefficients; beta is the standardized regression coefficient: beta is standardized according to $\mathrm{SD}_{\mathrm{y}}$. 
Table 29: Differential effects on students' grades across types of school - common model

\begin{tabular}{|c|c|c|c|c|c|}
\hline $\begin{array}{l}\text { Students' average grade in } \\
\text { German and Math }\end{array}$ & b & SE & $p$ & beta & SE \\
\hline $\begin{array}{l}\text { Lower secondary education } \\
\text { (Hauptschulabschluss) }\end{array}$ & Ref. & & & & \\
\hline $\begin{array}{l}\text { Intermediate secondary edu- } \\
\text { cation (Mittlere Reife) }\end{array}$ & 0.024 & 0.051 & 0.643 & 0.024 & 0.052 \\
\hline $\begin{array}{l}\text { Higher secondary education } \\
\text { (Abitur) }\end{array}$ & 0.130 & 0.057 & 0.023 & 0.133 & 0.058 \\
\hline $\begin{array}{l}\text { Lower tertiary education } \\
\text { (Fachochschulabschluss) }\end{array}$ & 0.167 & 0.067 & 0.013 & 0.170 & 0.068 \\
\hline $\begin{array}{l}\text { Higher tertiary education } \\
\text { (Hochschulabschluss) }\end{array}$ & 0.296 & 0.063 & 0.000 & 0.302 & 0.064 \\
\hline Migration background & -0.172 & 0.033 & 0.000 & -0.175 & 0.034 \\
\hline Male & -0.075 & 0.032 & 0.020 & -0.076 & 0.033 \\
\hline Perceptual speed & 0.006 & 0.001 & 0.000 & 0.082 & 0.014 \\
\hline Reasoning & 0.079 & 0.007 & 0.000 & 0.210 & 0.019 \\
\hline Schools without tracking & -0.279 & 0.167 & 0.095 & -0.285 & 0.170 \\
\hline Lower and intermediate track & Ref. & & & & \\
\hline Higher track & -0.430 & 0.106 & 0.000 & -0.438 & 0.108 \\
\hline $\begin{array}{l}\text { Students' high cultural activi- } \\
\text { ties }\end{array}$ & 0.009 & 0.010 & 0.340 & 0.023 & 0.025 \\
\hline $\begin{array}{l}\text { Parents' high cultural activi- } \\
\text { ties }\end{array}$ & 0.017 & 0.011 & 0.135 & 0.045 & 0.029 \\
\hline Reading activities & 0.042 & 0.038 & 0.275 & 0.027 & 0.025 \\
\hline $\begin{array}{l}\text { Students' high cultural activi- } \\
\text { ties * schools without track- } \\
\text { ing }\end{array}$ & 0.009 & 0.021 & 0.680 & 0.023 & 0.053 \\
\hline $\begin{array}{l}\text { Students' high cultural activi- } \\
\text { ties * lower and intermediate } \\
\text { track }\end{array}$ & Ref. & & & & \\
\hline $\begin{array}{l}\text { Students' high cultural activi- } \\
\text { ties * higher track }\end{array}$ & 0.007 & 0.012 & 0.582 & 0.018 & 0.030 \\
\hline $\begin{array}{l}\text { Parents' high cultural activi- } \\
\text { ties * schools without track- } \\
\text { ing }\end{array}$ & -0.001 & 0.017 & 0.930 & -0.003 & 0.045 \\
\hline $\begin{array}{l}\text { Parents' high cultural activi- } \\
\text { ties * lower and intermediate } \\
\text { track }\end{array}$ & Ref. & & & & \\
\hline
\end{tabular}




\begin{tabular}{l|ll|l|ll}
\hline & b & SE & p & beta & SE \\
\hline $\begin{array}{l}\text { Parents' high cultural activi- } \\
\text { ties * higher track }\end{array}$ & 0.004 & 0.014 & 0.784 & 0.011 & 0.037 \\
$\begin{array}{l}\text { Reading activities * schools } \\
\text { without tracking }\end{array}$ & 0.145 & 0.067 & 0.030 & 0.094 & 0.044 \\
$\begin{array}{l}\text { Reading activities * lower and } \\
\text { intermediate track }\end{array}$ & Ref. & & & & \\
$\begin{array}{l}\text { Reading activities * higher } \\
\text { track }\end{array}$ & 0.068 & 0.052 & 0.185 & 0.044 & 0.034 \\
Intercept & -0.856 & 0.114 & 0.000 & & \\
\\
Source: own calculations based on NEPS SC3 7.0.0
\end{tabular}

Source: own calculations based on NEPS SC3 7.0.0

Notes: $R^{2}=0.092 ; n=4,924 ; b$ is the unstandardized regression coefficients; beta is the standardized regression coefficient: If $y$ and $x$ are continuous, beta is standardized according to $S D_{y}$ and $S D_{x}$. If $y$ is continuous and $x$ binary, beta is standardized according to $\mathrm{SD}_{\mathrm{y}}$. 
Table 30: Direct, indirect, and total effects of parental education on mediating variables - revised model

\begin{tabular}{|c|c|c|c|c|c|c|c|}
\hline & & via & \multicolumn{3}{|c|}{ Unstandardized } & $\begin{array}{l}\text { Standa } \\
\text { beta }\end{array}$ & $\begin{array}{l}\text { zed } \\
\text { SE }\end{array}$ \\
\hline \multicolumn{8}{|l|}{ Students' high cultural practices } \\
\hline Intermediate secondary education & $\begin{array}{l}\text { Direct effect } \\
\text { Indirect effect } \\
\text { Total effect }\end{array}$ & $\rightarrow$ Parents' high cultural practices & $\begin{array}{l}-0.174 \\
0.133 \\
-0.041\end{array}$ & $\begin{array}{l}0.127 \\
0.024 \\
0.129\end{array}$ & $\begin{array}{l}0.171 \\
0.000 \\
0.749\end{array}$ & $\begin{array}{l}-0.070 \\
0.054 \\
-0.017\end{array}$ & $\begin{array}{l}0.051 \\
0.010 \\
0.052\end{array}$ \\
\hline Higher secondary education & $\begin{array}{l}\text { Direct effect } \\
\text { Indirect effect } \\
\text { Total effect }\end{array}$ & $\rightarrow$ Parents' high cultural practices & $\begin{array}{l}-0.263 \\
0.270 \\
0.007\end{array}$ & $\begin{array}{l}0.136 \\
0.032 \\
0.138\end{array}$ & $\begin{array}{l}0.053 \\
0.000 \\
0.959\end{array}$ & $\begin{array}{l}-0.106 \\
0.109 \\
0.003\end{array}$ & $\begin{array}{l}0.055 \\
0.013 \\
0.056\end{array}$ \\
\hline Lower tertiary education & $\begin{array}{l}\text { Direct effect } \\
\text { Indirect effect } \\
\text { Total effect }\end{array}$ & $\rightarrow$ Parents' high cultural practices & $\begin{array}{l}-0.059 \\
0.424 \\
0.365\end{array}$ & $\begin{array}{l}0.172 \\
0.046 \\
0.171\end{array}$ & $\begin{array}{l}0.732 \\
0.000 \\
0.033\end{array}$ & $\begin{array}{l}-0.024 \\
0.171 \\
0.147\end{array}$ & $\begin{array}{l}0.070 \\
0.019 \\
0.069\end{array}$ \\
\hline Higher tertiary education & $\begin{array}{l}\text { Direct effect } \\
\text { Indirect effect } \\
\text { Total effect }\end{array}$ & $\rightarrow$ Parents' high cultural practices & $\begin{array}{l}0.198 \\
0.630 \\
0.828\end{array}$ & $\begin{array}{l}0.158 \\
0.054 \\
0.161\end{array}$ & $\begin{array}{l}0.211 \\
0.000 \\
0.000\end{array}$ & $\begin{array}{l}0.080 \\
0.255 \\
0.335\end{array}$ & $\begin{array}{l}0.064 \\
0.022 \\
0.065\end{array}$ \\
\hline Attitudes towards reading & & & & & & & \\
\hline Intermediate secondary education & $\begin{array}{l}\text { Direct effect } \\
\text { Indirect effect }\end{array}$ & $\rightarrow$ Number of books in the home & $\begin{array}{l}0.146 \\
0.159\end{array}$ & $\begin{array}{l}0.148 \\
0.025\end{array}$ & $\begin{array}{l}0.321 \\
0.000\end{array}$ & $\begin{array}{l}0.053 \\
0.057\end{array}$ & $\begin{array}{l}0.053 \\
0.009\end{array}$ \\
\hline
\end{tabular}




\begin{tabular}{|c|c|c|c|c|c|c|c|}
\hline & & \multirow[t]{2}{*}{ via } & \multicolumn{3}{|c|}{ Unstandardized } & \multicolumn{2}{|c|}{ Standardized } \\
\hline & & & b & SE & $\mathbf{p}$ & beta & SE \\
\hline & Total effect & & 0.305 & 0.145 & 0.035 & 0.110 & 0.052 \\
\hline Higher secondary education & $\begin{array}{l}\text { Direct effect } \\
\text { Indirect effect } \\
\text { Total effect }\end{array}$ & $\rightarrow$ Number of books in the home & $\begin{array}{l}0.344 \\
0.300 \\
0.644\end{array}$ & $\begin{array}{l}0.160 \\
0.043 \\
0.155\end{array}$ & $\begin{array}{l}0.032 \\
0.000 \\
0.000\end{array}$ & $\begin{array}{r}0.124 \\
0.108 \\
0.233\end{array}$ & $\begin{array}{l}0.058 \\
0.016 \\
0.056\end{array}$ \\
\hline Lower tertiary education & $\begin{array}{l}\text { Direct effect } \\
\text { Indirect effect } \\
\text { Total effect }\end{array}$ & $\rightarrow$ Number of books in the home & $\begin{array}{l}0.437 \\
0.402 \\
0.839\end{array}$ & $\begin{array}{l}0.184 \\
0.057 \\
0.174\end{array}$ & $\begin{array}{l}0.017 \\
0.000 \\
0.000\end{array}$ & $\begin{array}{l}0.158 \\
0.145 \\
0.303\end{array}$ & $\begin{array}{l}0.066 \\
0.020 \\
0.063\end{array}$ \\
\hline Higher tertiary education & $\begin{array}{l}\text { Direct effect } \\
\text { Indirect effect } \\
\text { Total effect }\end{array}$ & $\rightarrow$ Number of books in the home & $\begin{array}{l}0.564 \\
0.542 \\
1.105\end{array}$ & $\begin{array}{l}0.169 \\
0.075 \\
0.154\end{array}$ & $\begin{array}{l}0.001 \\
0.000 \\
0.000\end{array}$ & $\begin{array}{l}0.204 \\
0.195 \\
0.399\end{array}$ & $\begin{array}{l}0.061 \\
0.027 \\
0.055\end{array}$ \\
\hline \multicolumn{8}{|l|}{ Reading practices } \\
\hline Intermediate secondary education & $\begin{array}{l}\text { Direct effect } \\
\text { Indirect effect } \\
\text { Total effect }\end{array}$ & $\begin{array}{l}\rightarrow \text { Number of books in the home } \rightarrow \text { attitudes towards } \\
\text { reading }\end{array}$ & $\begin{array}{l}0.024 \\
0.036 \\
0.061\end{array}$ & $\begin{array}{l}0.027 \\
0.017 \\
0.030\end{array}$ & $\begin{array}{l}0.366 \\
0.036 \\
0.040\end{array}$ & $\begin{array}{l}0.038 \\
0.057 \\
0.095\end{array}$ & $\begin{array}{l}0.042 \\
0.027 \\
0.046\end{array}$ \\
\hline Higher secondary education & $\begin{array}{l}\text { Direct effect } \\
\text { Indirect effect }\end{array}$ & $\begin{array}{l}\rightarrow \text { Number of books in the home } \rightarrow \text { attitudes towards } \\
\text { reading }\end{array}$ & $\begin{array}{l}0.030 \\
0.077\end{array}$ & $\begin{array}{l}0.030 \\
0.019\end{array}$ & $\begin{array}{l}0.317 \\
0.000\end{array}$ & $\begin{array}{l}0.048 \\
0.121\end{array}$ & $\begin{array}{l}0.048 \\
0.029\end{array}$ \\
\hline
\end{tabular}




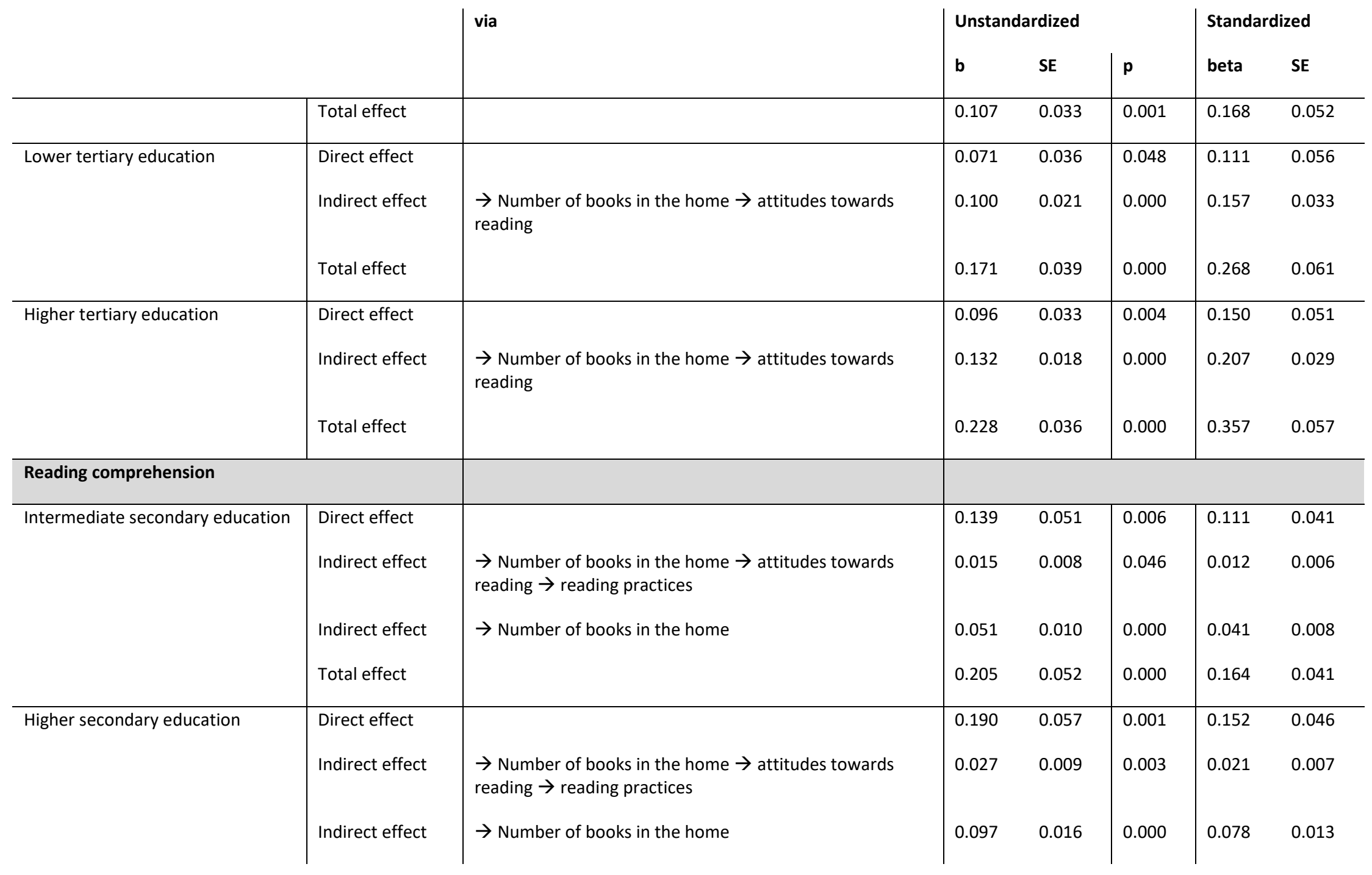




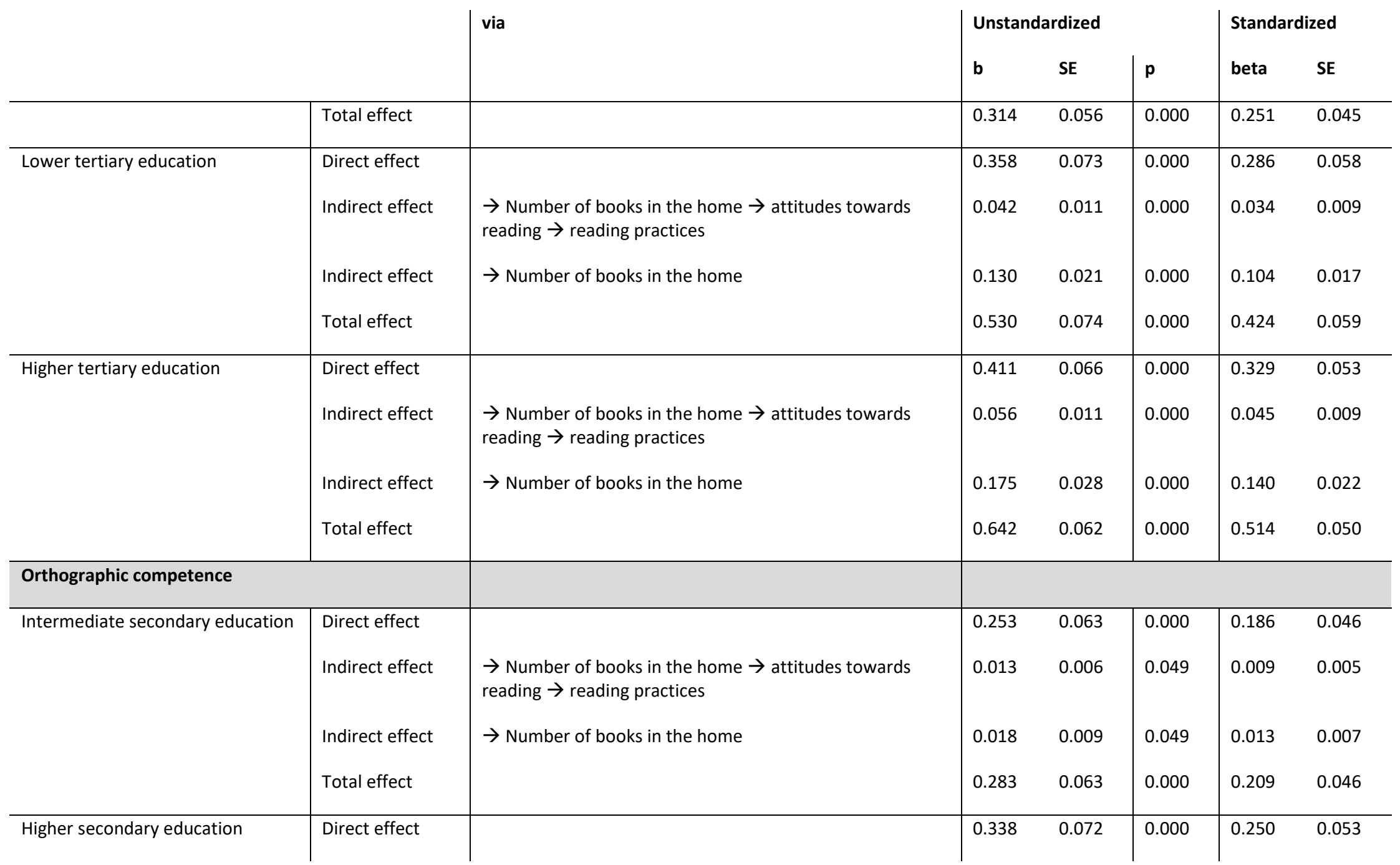




\begin{tabular}{|c|c|c|c|c|c|c|c|}
\hline & & via & Unsta & rdized & & Stand & \\
\hline & & & b & SE & $\mathbf{p}$ & beta & SE \\
\hline & Indirect effect & $\begin{array}{l}\rightarrow \text { Number of books in the home } \rightarrow \text { attitudes towards } \\
\text { reading } \rightarrow \text { reading practices }\end{array}$ & 0.022 & 0.008 & 0.004 & 0.017 & 0.006 \\
\hline & Indirect effect & $\rightarrow$ Number of books in the home & 0.034 & 0.017 & 0.045 & 0.025 & 0.012 \\
\hline & Total effect & & 0.395 & 0.070 & 0.000 & 0.291 & 0.052 \\
\hline Lower tertiary education & Direct effect & & 0.323 & 0.089 & 0.000 & 0.238 & 0.066 \\
\hline & Indirect effect & $\begin{array}{l}\rightarrow \text { Number of books in the home } \rightarrow \text { attitudes towards } \\
\text { reading } \rightarrow \text { reading practices }\end{array}$ & 0.036 & 0.009 & 0.000 & 0.026 & 0.007 \\
\hline & Indirect effect & $\rightarrow$ Number of books in the home & 0.045 & 0.023 & 0.044 & 0.033 & 0.017 \\
\hline & Total effect & & 0.393 & 0.089 & 0.000 & 0.290 & 0.066 \\
\hline Higher tertiary education & Direct effect & & 0.430 & 0.078 & 0.000 & 0.317 & 0.058 \\
\hline & Indirect effect & $\begin{array}{l}\rightarrow \text { Number of books in the home } \rightarrow \text { attitudes towards } \\
\text { reading } \rightarrow \text { reading practices }\end{array}$ & 0.048 & 0.010 & 0.000 & 0.035 & 0.008 \\
\hline & Indirect effect & $\rightarrow$ Number of books in the home & 0.061 & 0.030 & 0.044 & 0.045 & 0.022 \\
\hline & Total effect & & 0.538 & 0.076 & 0.000 & 0.397 & 0.056 \\
\hline
\end{tabular}

Source: own calculations based on NEPS SC3 7.0.0

Notes: $\mathrm{n}=4,924$; lower secondary education is the reference category; $\mathrm{b}$ is the unstandardized regression coefficients; beta is the standardized regression coefficient: beta is standardized according to $\mathrm{SD}_{\mathrm{y}}$. 
Table 31: Direct, indirect, and total effects of parental education on students' grades - revised model

\begin{tabular}{|c|c|c|c|c|c|c|c|}
\hline & & via & \multicolumn{3}{|c|}{ Unstandardized } & $\begin{array}{l}\text { Standa } \\
\text { beta }\end{array}$ & $\begin{array}{l}\text { zed } \\
\text { SE }\end{array}$ \\
\hline \multicolumn{8}{|l|}{$\begin{array}{l}\text { Students' average grade } \\
\text { (school without tracking) }\end{array}$} \\
\hline Intermediate secondary education & $\begin{array}{l}\text { Direct effect } \\
\text { Total indirect effect } \\
\text { Indirect effect } \\
\text { Indirect effect } \\
\text { Indirect effect } \\
\text { Indirect effect } \\
\text { Total effect }\end{array}$ & $\begin{array}{l}\rightarrow \text { Parents' high cultural participation } \\
\rightarrow \text { Students' high cultural participation } \\
\rightarrow \text { Reading comprehension } \\
\rightarrow \text { Orthographic competence }\end{array}$ & $\begin{array}{l}-0.099 \\
0.095 \\
-0.002 \\
-0.001 \\
0.034 \\
0.064 \\
-0.004\end{array}$ & $\begin{array}{l}0.110 \\
0.022 \\
0.008 \\
0.002 \\
0.011 \\
0.017 \\
0.109\end{array}$ & $\begin{array}{l}0.369 \\
0.000 \\
0.836 \\
0.760 \\
0.002 \\
0.000 \\
0.969\end{array}$ & $\begin{array}{l}-0.101 \\
0.097 \\
-0.002 \\
-0.001 \\
0.034 \\
0.065 \\
-0.004\end{array}$ & $\begin{array}{l}0.113 \\
0.022 \\
0.008 \\
0.002 \\
0.011 \\
0.017 \\
0.111\end{array}$ \\
\hline Higher secondary education & $\begin{array}{l}\text { Direct effect } \\
\text { Total indirect effect } \\
\text { Indirect effect } \\
\text { Indirect effect } \\
\text { Indirect effect } \\
\text { Indirect effect } \\
\text { Total effect }\end{array}$ & $\begin{array}{l}\rightarrow \text { Parents' high cultural participation } \\
\rightarrow \text { Students' high cultural participation } \\
\rightarrow \text { Reading comprehension } \\
\rightarrow \text { Orthographic competence }\end{array}$ & $\begin{array}{l}0.010 \\
0.137 \\
-0.003 \\
0.000 \\
0.052 \\
0.089 \\
0.147\end{array}$ & $\begin{array}{l}0.100 \\
0.030 \\
0.017 \\
0.002 \\
0.015 \\
0.021 \\
0.103\end{array}$ & $\begin{array}{l}0.920 \\
0.000 \\
0.836 \\
0.959 \\
0.000 \\
0.000 \\
0.154\end{array}$ & $\begin{array}{l}0.010 \\
0.140 \\
-0.003 \\
0.000 \\
0.053 \\
0.090 \\
0.150\end{array}$ & $\begin{array}{l}0.102 \\
0.031 \\
0.017 \\
0.002 \\
0.015 \\
0.021 \\
0.105\end{array}$ \\
\hline Lower tertiary education & Direct effect & & 0.214 & 0.153 & 0.162 & 0.218 & 0.156 \\
\hline
\end{tabular}




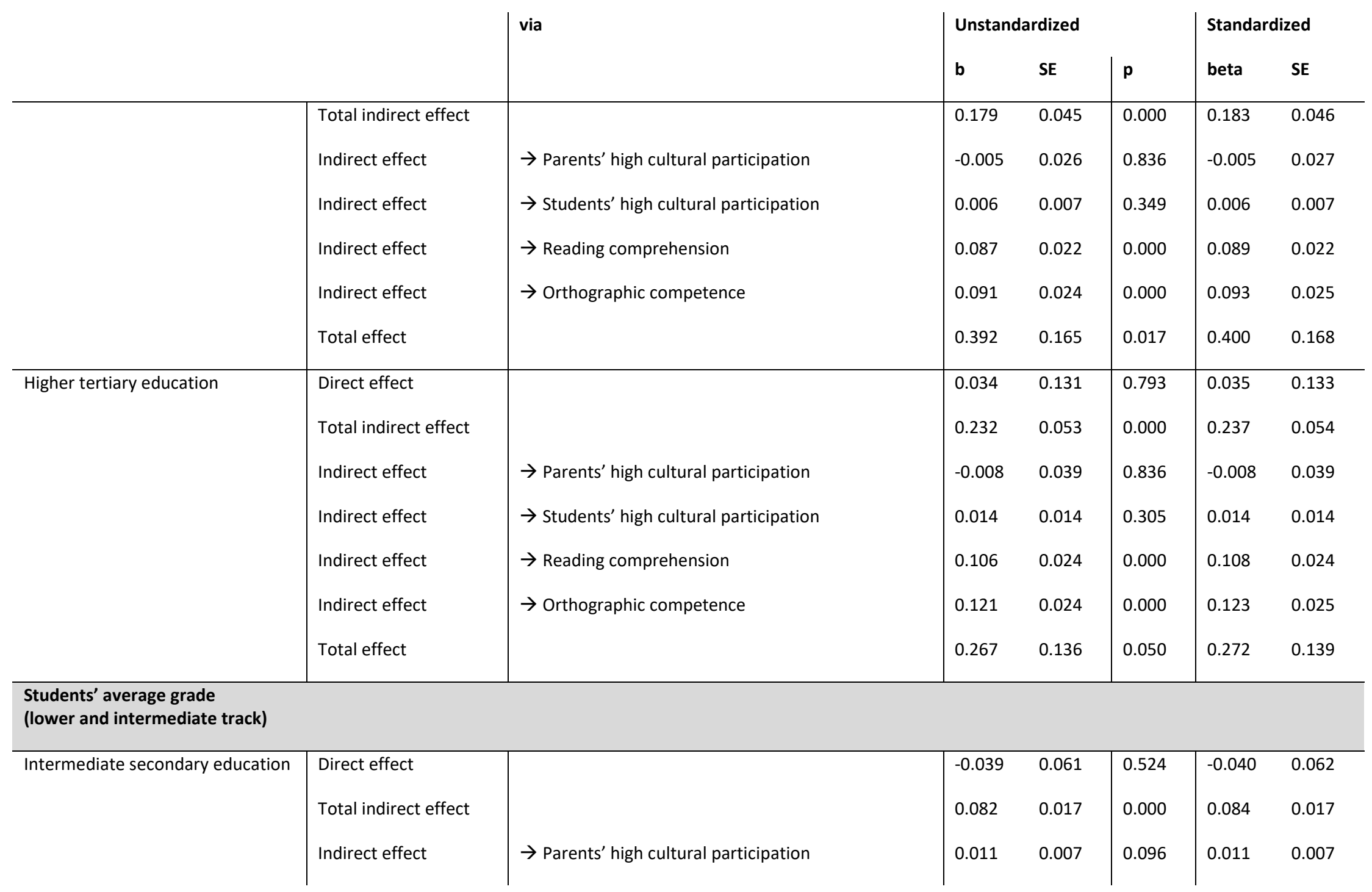




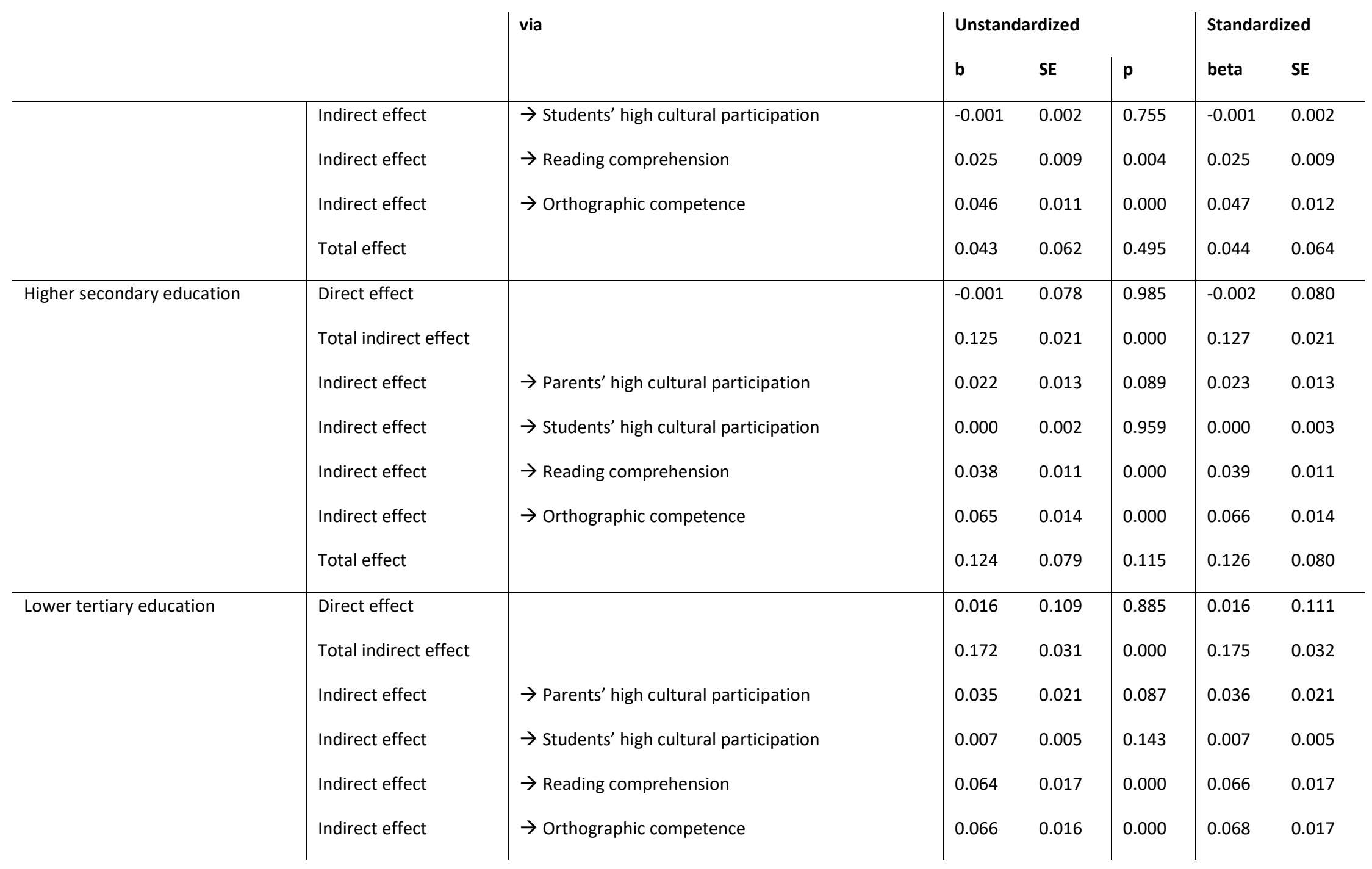




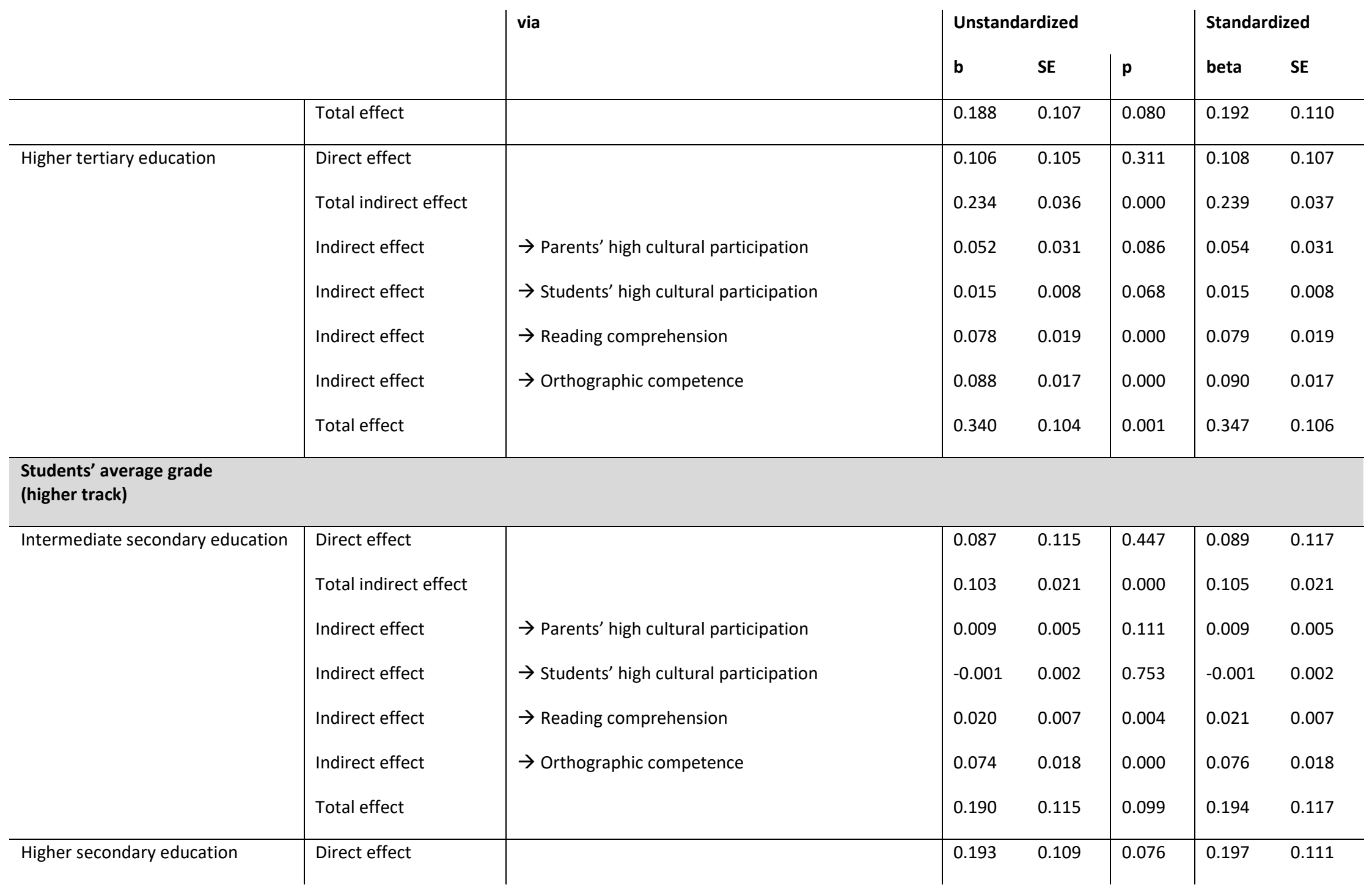




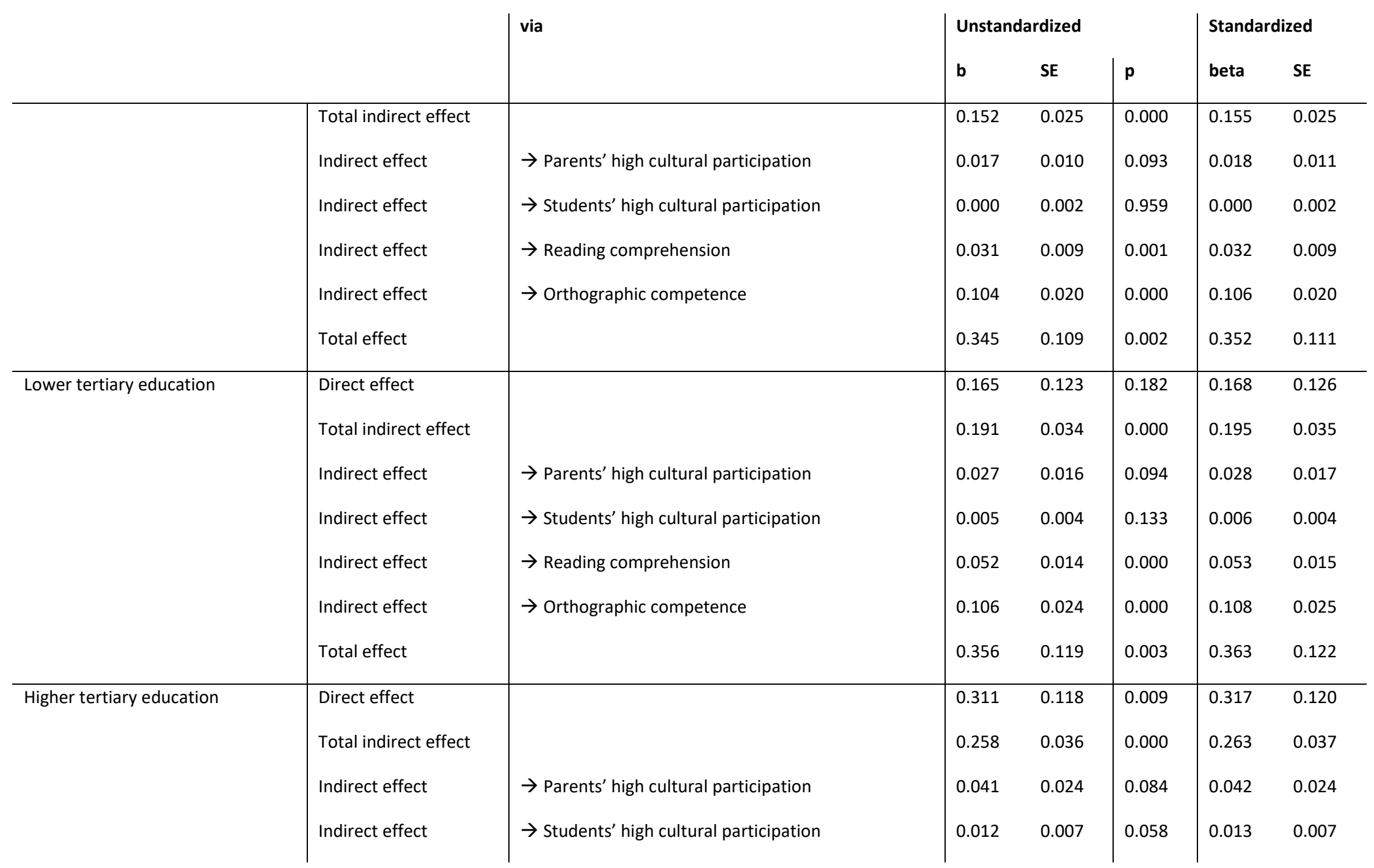




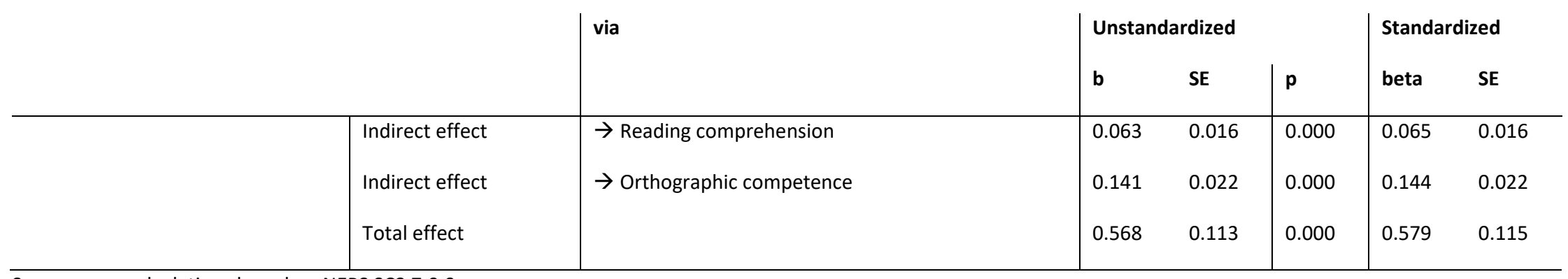

Source: own calculations based on NEPS SC3 7.0.0

Notes: $n=4,924$; lower secondary education is the reference category; $b$ is the unstandardized regression coefficients; beta is the standardized regression coefficient: beta is standardized according to $\mathrm{SD}_{\mathrm{y}}$. 
Table 32: Differential effects on students' grades across types of school - revised model

\begin{tabular}{|c|c|c|c|c|c|}
\hline $\begin{array}{l}\text { Students' average grade in } \\
\text { German and Math }\end{array}$ & b & SE & $\mathbf{p}$ & beta & SE \\
\hline $\begin{array}{l}\text { Lower secondary education } \\
\text { (Hauptschulabschluss) }\end{array}$ & Ref. & & & & \\
\hline $\begin{array}{l}\text { Intermediate secondary edu- } \\
\text { cation (Mittlere Reife) }\end{array}$ & $-0,040$ & 0,050 & 0,429 & $-0,041$ & 0,051 \\
\hline $\begin{array}{l}\text { Higher secondary education } \\
\text { (Abitur) }\end{array}$ & 0,040 & 0,054 & 0,460 & 0,041 & 0,055 \\
\hline $\begin{array}{l}\text { Lower tertiary education } \\
\text { (Fachochschulabschluss) }\end{array}$ & 0,060 & 0,064 & 0,352 & 0,061 & 0,065 \\
\hline $\begin{array}{l}\text { Higher tertiary education } \\
\text { (Hochschulabschluss) }\end{array}$ & 0,151 & 0,061 & 0,014 & 0,154 & 0,062 \\
\hline Migration background & $-0,123$ & 0,031 & 0,000 & $-0,125$ & 0,032 \\
\hline Male & 0,010 & 0,030 & 0,729 & 0,010 & 0,031 \\
\hline Perceptual speed & 0,005 & 0,001 & 0,000 & 0,068 & 0,014 \\
\hline Reasoning & 0,042 & 0,007 & 0,000 & 0,111 & 0,019 \\
\hline Schools without tracking & 0,055 & 0,151 & 0,716 & 0,056 & 0,154 \\
\hline Lower and intermediate track & Ref. & & & & \\
\hline Higher track & $-0,569$ & 0,102 & 0,000 & $-0,580$ & 0,104 \\
\hline $\begin{array}{l}\text { Students' high cultural activi- } \\
\text { ties }\end{array}$ & 0,019 & 0,009 & 0,036 & 0,048 & 0,023 \\
\hline $\begin{array}{l}\text { Parents' high cultural activi- } \\
\text { ties }\end{array}$ & 0,015 & 0,011 & 0,174 & 0,040 & 0,029 \\
\hline Reading comprehension & 0,103 & 0,026 & 0,000 & 0,131 & 0,033 \\
\hline Orthographic competence & 0,157 & 0,025 & 0,000 & 0,217 & 0,035 \\
\hline $\begin{array}{l}\text { Students' high cultural activi- } \\
\text { ties * schools without track- } \\
\text { ing }\end{array}$ & $-0,002$ & 0,018 & 0,897 & $-0,005$ & 0,045 \\
\hline $\begin{array}{l}\text { Students' high cultural activi- } \\
\text { ties * lower and intermediate } \\
\text { track }\end{array}$ & Ref. & & & & \\
\hline $\begin{array}{l}\text { Students' high cultural activi- } \\
\text { ties * higher track }\end{array}$ & $-0,004$ & 0,012 & 0,715 & $-0,010$ & 0,030 \\
\hline $\begin{array}{l}\text { Parents' high cultural activi- } \\
\text { ties * schools without track- } \\
\text { ing }\end{array}$ & $-0,021$ & 0,017 & 0,225 & $-0,055$ & 0,045 \\
\hline
\end{tabular}




\begin{tabular}{|c|c|c|c|c|c|}
\hline & b & SE & $p$ & beta & SE \\
\hline $\begin{array}{l}\text { Parents' high cultural activi- } \\
\text { ties * lower and intermediate } \\
\text { track }\end{array}$ & Ref. & & & & \\
\hline $\begin{array}{l}\text { Parents' high cultural activi- } \\
\text { ties * higher track }\end{array}$ & 0,003 & 0,014 & 0,817 & 0,008 & 0,037 \\
\hline $\begin{array}{l}\text { Reading comprehension* } \\
\text { schools without tracking }\end{array}$ & 0,057 & 0,043 & 0,185 & 0,073 & 0,055 \\
\hline $\begin{array}{l}\text { Reading comprehension * } \\
\text { lower and intermediate track }\end{array}$ & Ref. & & & & \\
\hline $\begin{array}{l}\text { Reading comprehension * } \\
\text { higher track }\end{array}$ & 0,010 & 0,035 & 0,784 & 0,013 & 0,045 \\
\hline $\begin{array}{l}\text { Orthographic competence * } \\
\text { schools without tracking }\end{array}$ & 0,065 & 0,039 & 0,097 & 0,090 & 0,054 \\
\hline $\begin{array}{l}\text { Orthographic competence } * \\
\text { lower and intermediate track }\end{array}$ & Ref. & & & & \\
\hline $\begin{array}{l}\text { Orthographic competence * } \\
\text { higher track }\end{array}$ & 0,114 & 0,032 & 0,000 & 0,158 & 0,044 \\
\hline Intercept & $-0,485$ & 0,110 & 0,000 & & \\
\hline
\end{tabular}

Source: own calculations based on NEPS SC3 7.0.0

Notes: $R^{2}=0.191 ; n=4,924 ; b$ is the unstandardized regression coefficients; beta is the standardized regression coefficient: If $y$ and $x$ are continuous, beta is standardized according to $\mathrm{SD}_{\mathrm{y}}$ and $\mathrm{SD}_{\mathrm{x}}$. If $\mathrm{y}$ is continuous and $\mathrm{x}$ binary, beta is standardized according to $\mathrm{SD}_{\mathrm{y}}$. 
Table 33: Cross table: Parental education and type of school

\begin{tabular}{l|lll} 
Parental education & School without tracking & $\begin{array}{l}\text { Lower and intermediate } \\
\text { track }\end{array}$ & Higher track \\
\hline Lower secondary & 62 & 311 & 59 \\
Intermediate secondary & 178 & 600 & 465 \\
Higher secondary & 99 & 242 & 451 \\
Lower tertiary & 57 & 77 & 235 \\
Higher tertiary & 112 & 106 & 599 \\
Missing value & 169 & 646 & 456
\end{tabular}

Source: own calculations based on NEPS SC3 7.0.0

Table 34: Pairwise correlations of the observed data

\begin{tabular}{|c|c|c|c|c|c|c|c|c|c|c|}
\hline & $x 2$ & $x 3$ & $x 4$ & $x 5$ & $x 6$ & $x 7$ & $x 8$ & c3 & c4 & $x 9$ \\
\hline$x 2$ & 1.0000 & & & & & & & & & \\
\hline$x 3$ & 0.2948 & 1.0000 & & & & & & & & \\
\hline$x 4$ & 0.4691 & 0.1386 & 1.0000 & & & & & & & \\
\hline$x 5$ & 0.1818 & 0.2273 & 0.2188 & 1.0000 & & & & & & \\
\hline$x 6$ & 0.1131 & 0.1709 & 0.1692 & 0.5391 & 1.0000 & & & & & \\
\hline$x 7$ & 0.2591 & 0.0406 & 0.3094 & 0.2852 & 0.2342 & 1.0000 & & & & \\
\hline$x 8$ & 0.1933 & 0.0709 & 0.2338 & 0.2864 & 0.2006 & 0.5547 & 1.0000 & & & \\
\hline c3 & 0.0086 & 0.0194 & -0.0165 & 0.0283 & 0.0405 & 0.0830 & 0.0932 & 1.0000 & & \\
\hline c4 & 0.1865 & 0.0215 & 0.2400 & 0.1174 & 0.0874 & 0.4375 & 0.3400 & 0.1380 & 1.0000 & \\
\hline$\times 9$ & 0.1268 & 0.0688 & 0.1148 & 0.1237 & 0.0956 & 0.2840 & 0.3194 & 0.0944 & 0.2157 & 1.0000 \\
\hline
\end{tabular}

Source: own calculations based on NEPS SC3 7.0.0

Note: bold values $p<0.05$ 